\title{
Four-Electron Reduction of Dioxygen on a Metal Surface: Models of Dissociative and Associative Mechanisms in a Homogeneous System
}

Hajime Kameo, ${ }^{*}, \dagger$ Shigeyoshi Sakaki, ${ }^{\S}$ Yasuhiro Ohki, ${ }^{\dagger, \|}$ Naoki Uehara, ${ }^{\dagger}$ Takuya Kosukegawa, ${ }^{\dagger}$ Hiroharu Suzuki,${ }^{\dagger}$ and Toshiro Takao ${ }^{*, \dagger}$

${ }^{\dagger}$ Department of Chemical Science and Engineering, School of Materials and Chemical Technology, Tokyo Institute of Technology, O-okayama 2-12-1, Meguro-ku, Tokyo 152-8552 Japan

$\$$ Department of Chemistry, Graduate School of Science, Osaka Prefecture University, Gakuen-cho, Naka-ku, Sakai, Osaka 599-8531 Japan

${ }^{\S}$ Fukui Institute for Fundamental Chemistry, Kyoto University, Takano-nishihiraki-cho 34-4, Sakyo-ku Kyoto 606-8103 Japan

\| Department of Chemistry, Graduate School of Science, Nagoya University, Furo-cho, Chikusa-ku, Nagoya 464-8602 Japan

\section{Table of content}

$\begin{array}{ll}\text { Isomerization of bis- } \mu_{3} \text {-oxo complex } & \mathrm{S} 2\end{array}$

X-ray Crystal Structure Determination $\quad$ S3

$\begin{array}{ll}\text { DFT calculations } & \text { S15 }\end{array}$

$\begin{array}{ll}\text { Cartesian Coordination } & \text { S32 }\end{array}$

$\begin{array}{lr}\text { NMR Spectra } & \text { S54 }\end{array}$

$\begin{array}{ll}\text { References } & \text { S63 }\end{array}$ 


\section{Isomerization of bis- $\mu 3-0 x o$ compound 3}

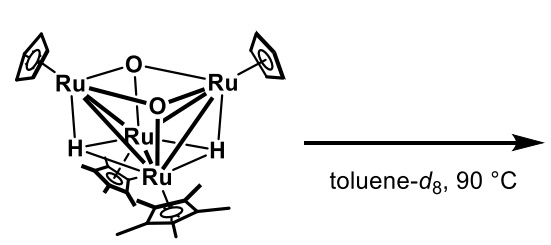

3b

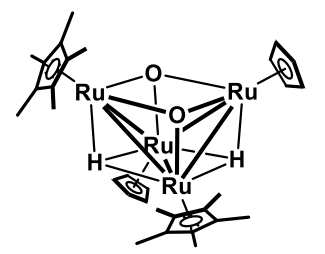

3a

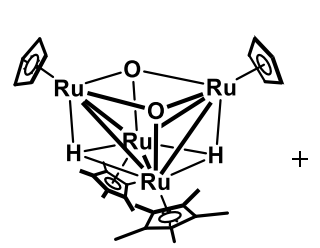

3b

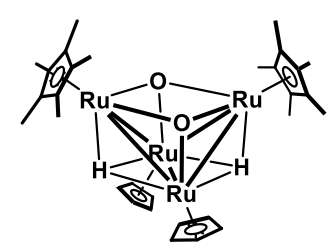

3c

$3 \mathbf{a} / \mathbf{3 b} / \mathbf{3 c}=13 / 11 / 76$

Complex $\mathbf{3 b}$ isomerized to the mixture of $\mathbf{3 a}, \mathbf{3 b}$, and $\mathbf{3 c}$ with a ratio of 13:11:76 upon heating at $90{ }^{\circ} \mathrm{C}$ for $60 \mathrm{~h}$ (Figure S1), and this ratio is the same in the isomerization products of $3 \mathbf{a}$ (see the manuscript).
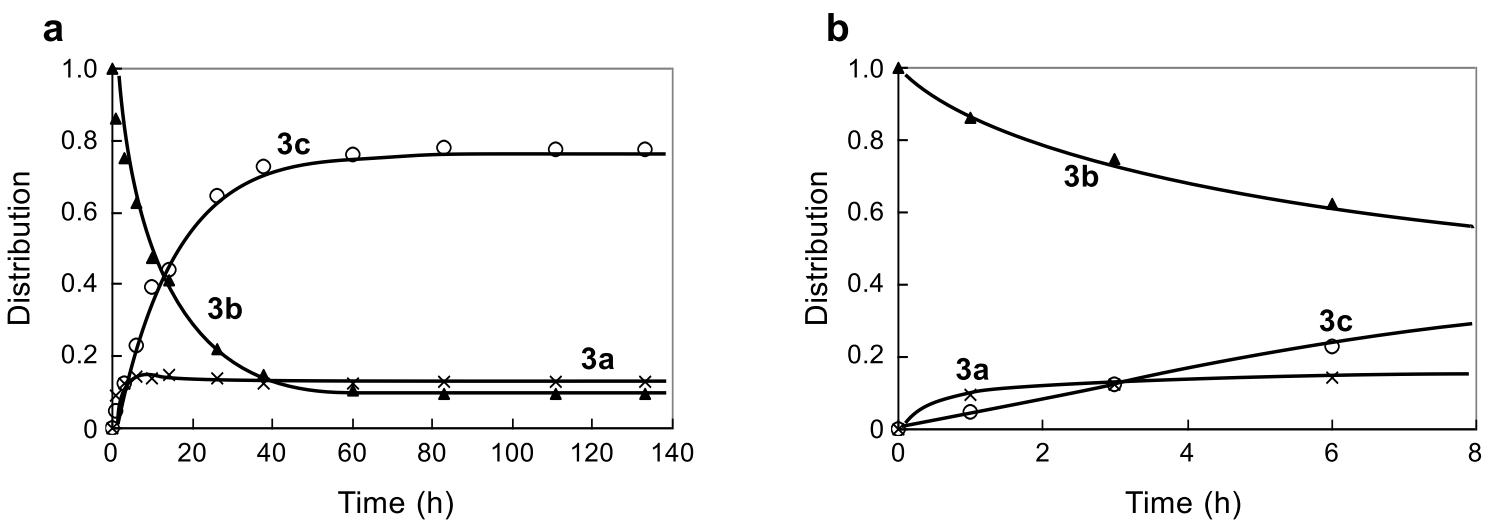

Figure S1 Isomerization of bis $\left(\mu_{3}-\mathrm{oxo}\right)$ complex $\mathbf{3 b}$ into $\mathbf{3 a}$ and $\mathbf{3} \mathbf{c}$ as a function of time. (a: whole reaction course; $\mathbf{b}$; initial stage) 

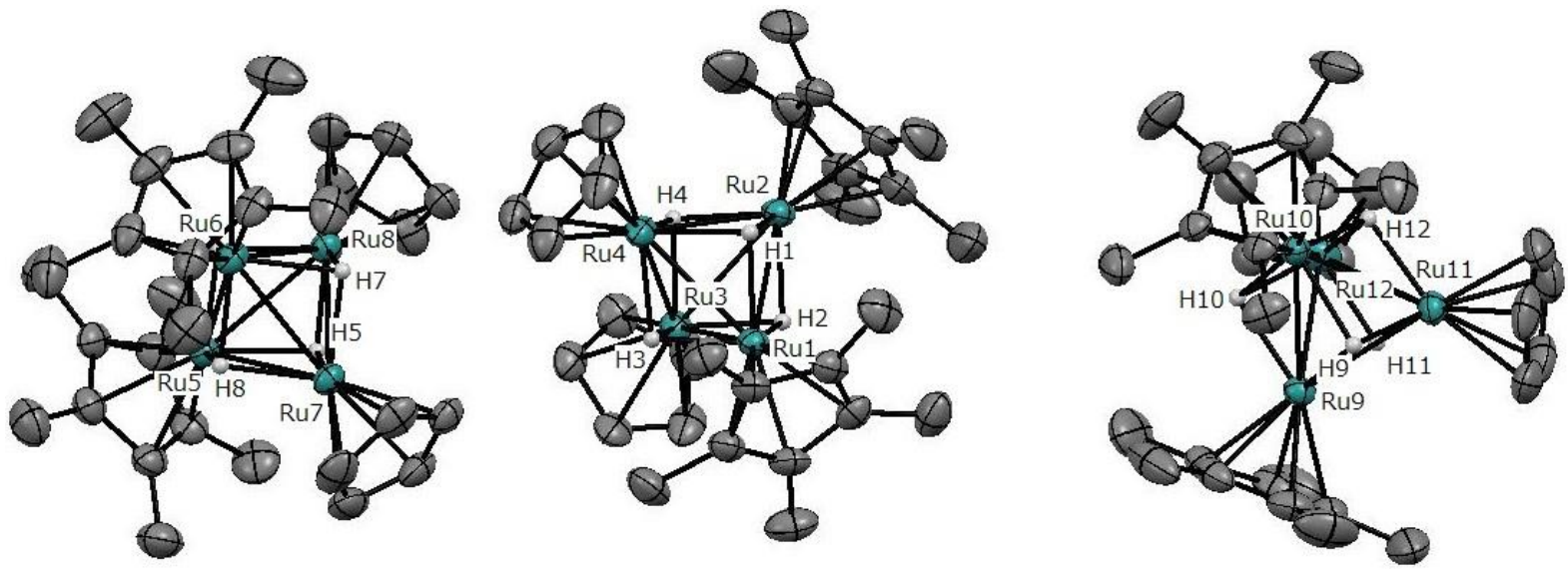

Figure S2 Molecular Structure of $\mathbf{1 b}$ (three independent molecules) 
Table S1. Crystal data and structure refinement for $\mathbf{1 b}$.

Identification code

Empirical formula

Formula weight

Temperature

Wavelength

Crystal system

Space group

Unit cell dimensions

Volume

$\mathrm{Z}$

Density (calculated)

Absorption coefficient

$\mathrm{F}(000)$

Crystal size

Theta range for data collection

Index ranges

Reflections collected

Independent reflections

Completeness to theta $=25.242^{\circ}$

Refinement method

Data / restraints / parameters

Goodness-of-fit on $\mathrm{F}^{2}$

Final R indices [I>2sigma(I)]

$\mathrm{R}$ indices (all data)

Extinction coefficient

Largest diff. peak and hole
$1 \mathrm{~b}$

C30 H44 Ru4

808.93

193(2) K

$0.71069 \AA$

Triclinic

$P-1$

$a=16.1544(5) \AA$

$\alpha=66.0450(7)^{\circ}$.

$\mathrm{b}=18.2708(4) \AA$

$\beta=63.6010(9)^{\circ}$.

$\mathrm{c}=19.1134(4) \AA$

$\gamma=67.0390(11)^{\circ}$.

4463.9(2) $\AA^{3}$

6

$1.805 \mathrm{Mg} / \mathrm{m}^{3}$

$2.007 \mathrm{~mm}^{-1}$

2400

$0.280 \times 0.240 \times 0.080 \mathrm{~mm}^{3}$

2.999 to $27.482^{\circ}$.

$-20<=\mathrm{h}<=20,-23<=\mathrm{k}<=22,-24<=\mathrm{l}<=24$

71593

$20363[\mathrm{R}(\mathrm{int})=0.0303]$

$99.4 \%$

Full-matrix least-squares on $\mathrm{F}^{2}$

20372 / 0 / 1002

1.038

$\mathrm{R} 1=0.0303, \mathrm{wR} 2=0.0723$

$\mathrm{R} 1=0.0380, \mathrm{wR} 2=0.0757$

$\mathrm{n} / \mathrm{a}$

2.151 and $-1.149 \mathrm{e} . \AA^{-3}$ 
X-ray Diffraction Analysis on 3a

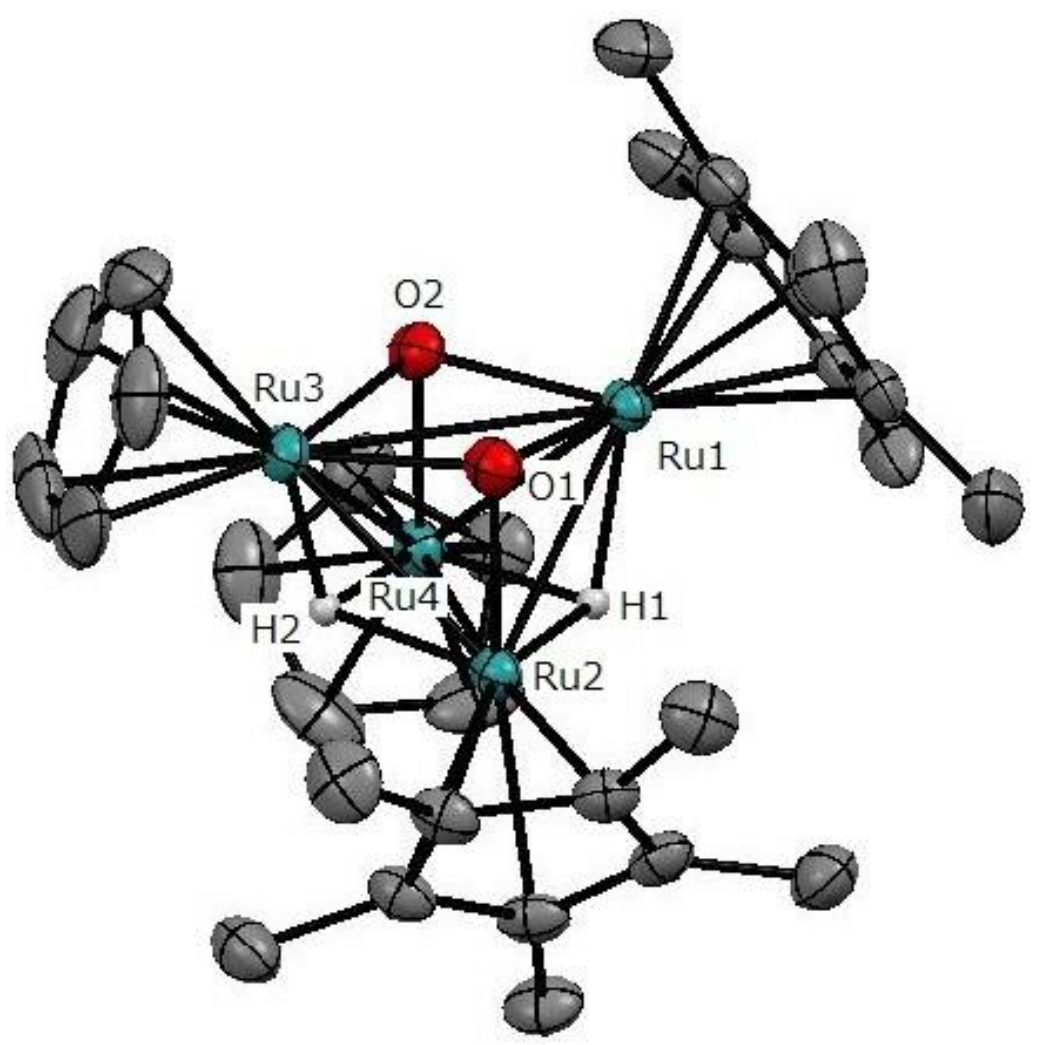

Figure S3 Molecular Structure of 3a 
Table S2. Crystal data and structure refinement for 3a.

Identification code

Empirical formula

Formula weight

Temperature

Wavelength

Crystal system

Space group

Unit cell dimensions

Volume

$\mathrm{Z}$

Density (calculated)

Absorption coefficient

$\mathrm{F}(000)$

Crystal size

Theta range for data collection

Index ranges

Reflections collected

Independent reflections

Completeness to theta $=25.242^{\circ}$

Refinement method

Data / restraints / parameters

Goodness-of-fit on $\mathrm{F}^{2}$

Final R indices [I>2sigma(I)]

$\mathrm{R}$ indices (all data)

Extinction coefficient

Largest diff. peak and hole 3a

C30 H42 O2 Ru4

838.91

173(2) K

$0.71069 \AA$

Monoclinic

$P 2{ }_{1} / c$

$\mathrm{a}=12.028(3) \AA$

$\alpha=90^{\circ}$.

$\mathrm{b}=15.816(6) \AA$

$\beta=105.215(10)^{\circ}$.

$\mathrm{c}=15.889(4) \AA$

$\gamma=90^{\circ}$.

$2916.6(15) \AA^{3}$

4

$1.910 \mathrm{Mg} / \mathrm{m}^{3}$

$2.057 \mathrm{~mm}^{-1}$

1656

$0.100 \times 0.100 \times 0.050 \mathrm{~mm}^{3}$

3.058 to $30.031^{\circ}$.

$0<=\mathrm{h}<=16,0<=\mathrm{k}<=22,-22<=\mathrm{l}<=21$

30575

$7890[\mathrm{R}(\mathrm{int})=0.0745]$

$92.4 \%$

Full-matrix least-squares on $\mathrm{F}^{2}$

$7890 / 0 / 343$

1.053

$\mathrm{R} 1=0.0508, \mathrm{wR} 2=0.1094$

$\mathrm{R} 1=0.0739, \mathrm{wR} 2=0.1209$

$\mathrm{n} / \mathrm{a}$

1.988 and -1.534 e. $\AA^{-3}$ 
X-ray Diffraction Analysis on 3b

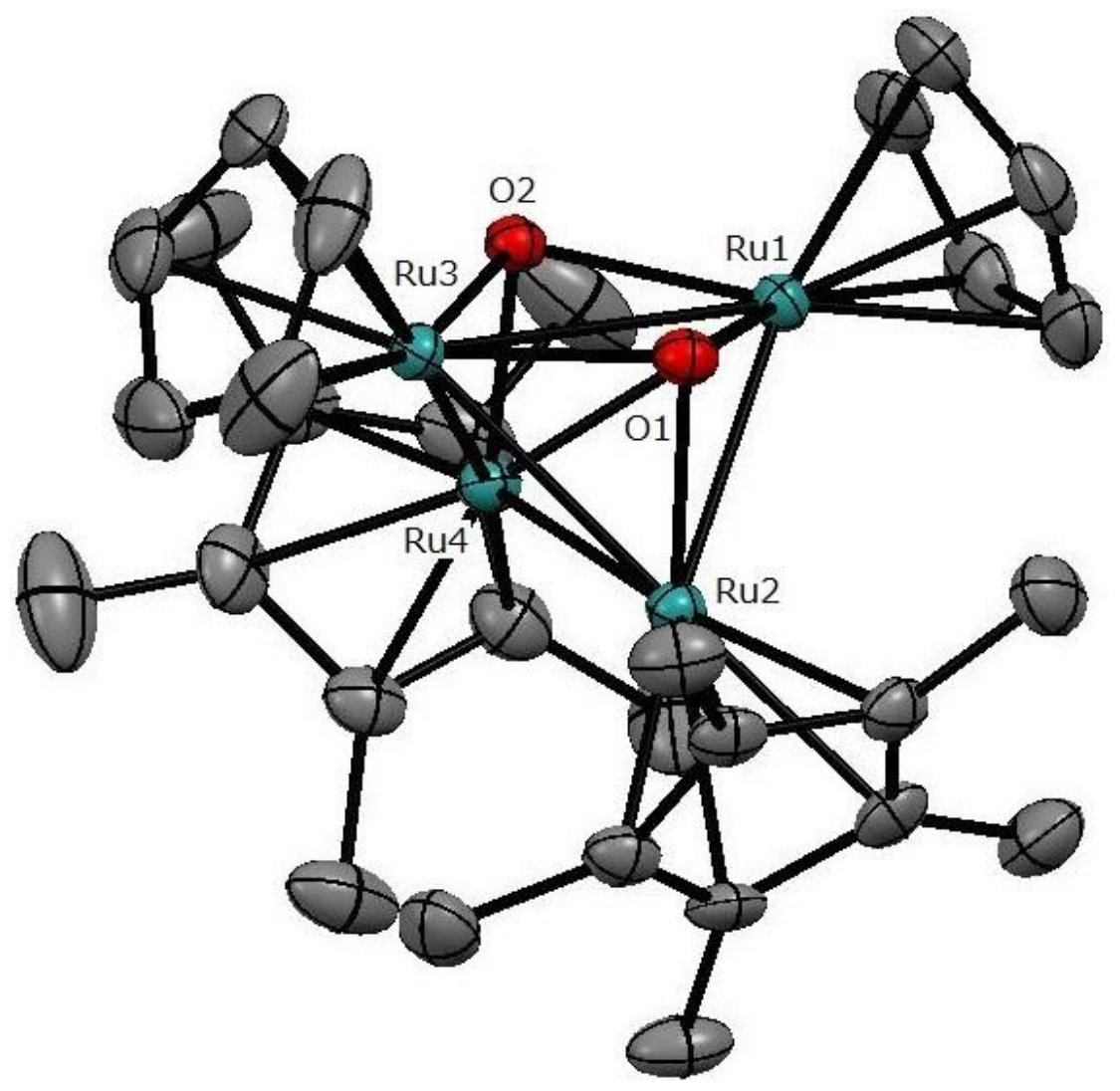

Figure S4 Molecular Structure of $\mathbf{3 b}$ 
Table S3. Crystal data and structure refinement for $\mathbf{3 b}$.

Identification code

Empirical formula

Formula weight

Temperature

Wavelength

Crystal system

Space group

Unit cell dimensions

Volume

$\mathrm{Z}$

Density (calculated)

Absorption coefficient

$\mathrm{F}(000)$

Crystal size

Theta range for data collection

Index ranges

Reflections collected

Independent reflections

Completeness to theta $=25.240^{\circ}$

Refinement method

Data / restraints / parameters

Goodness-of-fit on $\mathrm{F}^{2}$

Final R indices [I>2sigma(I)]

$\mathrm{R}$ indices (all data)

Extinction coefficient

Largest diff. peak and hole
$3 b$

C30 H46 O4 Ru4

874.95

123(2) K

$0.71069 \AA$

Monoclinic

$C 2 / c$

$\begin{array}{ll}\mathrm{a}=27.3279(16) \AA & \alpha=90^{\circ} . \\ \mathrm{b}=18.8489(13) \AA & \beta=129.752(9)^{\circ} . \\ \mathrm{c}=17.6359(10) \AA & \gamma=90^{\circ} .\end{array}$

6984.2(10) $\AA^{3}$

8

$1.664 \mathrm{Mg} / \mathrm{m}^{3}$

$1.725 \mathrm{~mm}^{-1}$

3472

$0.490 \times 0.200 \times 0.190 \mathrm{~mm}^{3}$

3.005 to $27.479^{\circ}$.

$-33<=\mathrm{h}<=35,-24<=\mathrm{k}<=24,-22<=1<=21$

33935

$7987[\mathrm{R}(\mathrm{int})=0.0386]$

$99.8 \%$

Full-matrix least-squares on $\mathrm{F}^{2}$

7987 / 81 / 372

1.041

$\mathrm{R} 1=0.0377, \mathrm{wR} 2=0.1010$

$\mathrm{R} 1=0.0398, \mathrm{wR} 2=0.1038$

$\mathrm{n} / \mathrm{a}$

2.048 and -1.481 e. $\AA^{-3}$ 
X-ray Diffraction Analysis on 4a

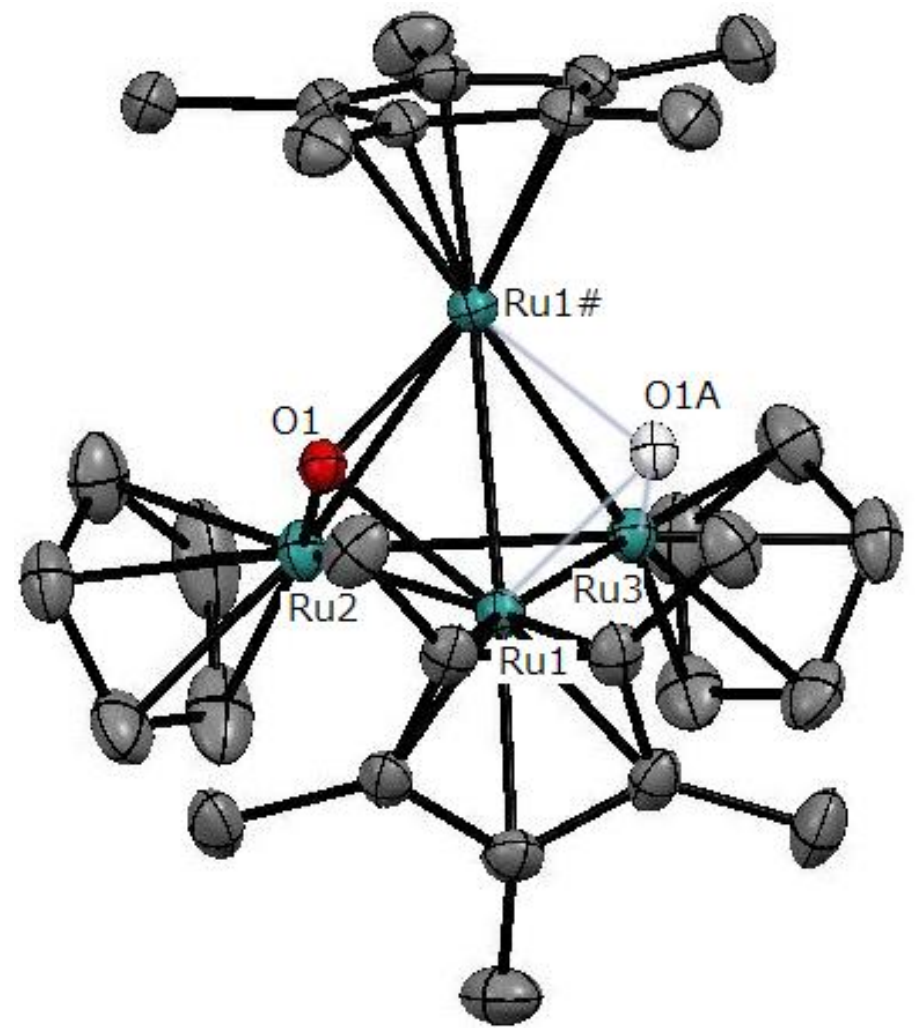

Figure S5 Molecular Structure of 4a. O1A shows disorder in the crystal $(\mathrm{O} 1: \mathrm{O} 1 \mathrm{~A}=$ $67: 33)$. 
Table S4. Crystal data and structure refinement for $\mathbf{4 a}$.

Identification code

Empirical formula

Formula weight

Temperature

Wavelength

Crystal system

Space group

Unit cell dimensions

Volume

$\mathrm{Z}$

Density (calculated)

Absorption coefficient

$\mathrm{F}(000)$

Crystal size

Theta range for data collection

Index ranges

Reflections collected

Independent reflections

Completeness to theta $=25.242^{\circ}$

Refinement method

Data / restraints / parameters

Goodness-of-fit on $\mathrm{F}^{2}$

Final R indices [I>2sigma(I)]

$\mathrm{R}$ indices (all data)

Extinction coefficient

Largest diff. peak and hole
$4 \mathrm{a}$

C30 H44 O Ru4

824.93

153(2) K

$0.71069 \AA$

Monoclinic

$C 2 / m$

$$
\begin{array}{ll}
\mathrm{a}=14.409(4) \AA & \alpha=90^{\circ} . \\
\mathrm{b}=17.410(6) \AA & \beta=102.110(15)^{\circ} . \\
\mathrm{c}=12.042(5) \AA & \gamma=90^{\circ} .
\end{array}
$$

4463.9(2) $\AA^{3}$

6

$1.805 \mathrm{Mg} / \mathrm{m}^{3}$

$2.007 \mathrm{~mm}^{-1}$

2400

$0.280 \times 0.240 \times 0.080 \mathrm{~mm}^{3}$

3.042 to $30.023^{\circ}$.

$0<=\mathrm{h}<=20,0<=\mathrm{k}<=24,-16<=1<=16$

17502

$4414[\mathrm{R}(\mathrm{int})=0.0312]$

$99.2 \%$

Full-matrix least-squares on $\mathrm{F}^{2}$

4414 / 0 / 183

1.018

$\mathrm{R} 1=0.0241, \mathrm{wR} 2=0.0592$

$\mathrm{R} 1=0.0274, \mathrm{wR} 2=0.0610$

$\mathrm{n} / \mathrm{a}$

0.744 and -1.208 e. $\AA^{-3}$ 
X-ray Diffraction Analysis on 4b

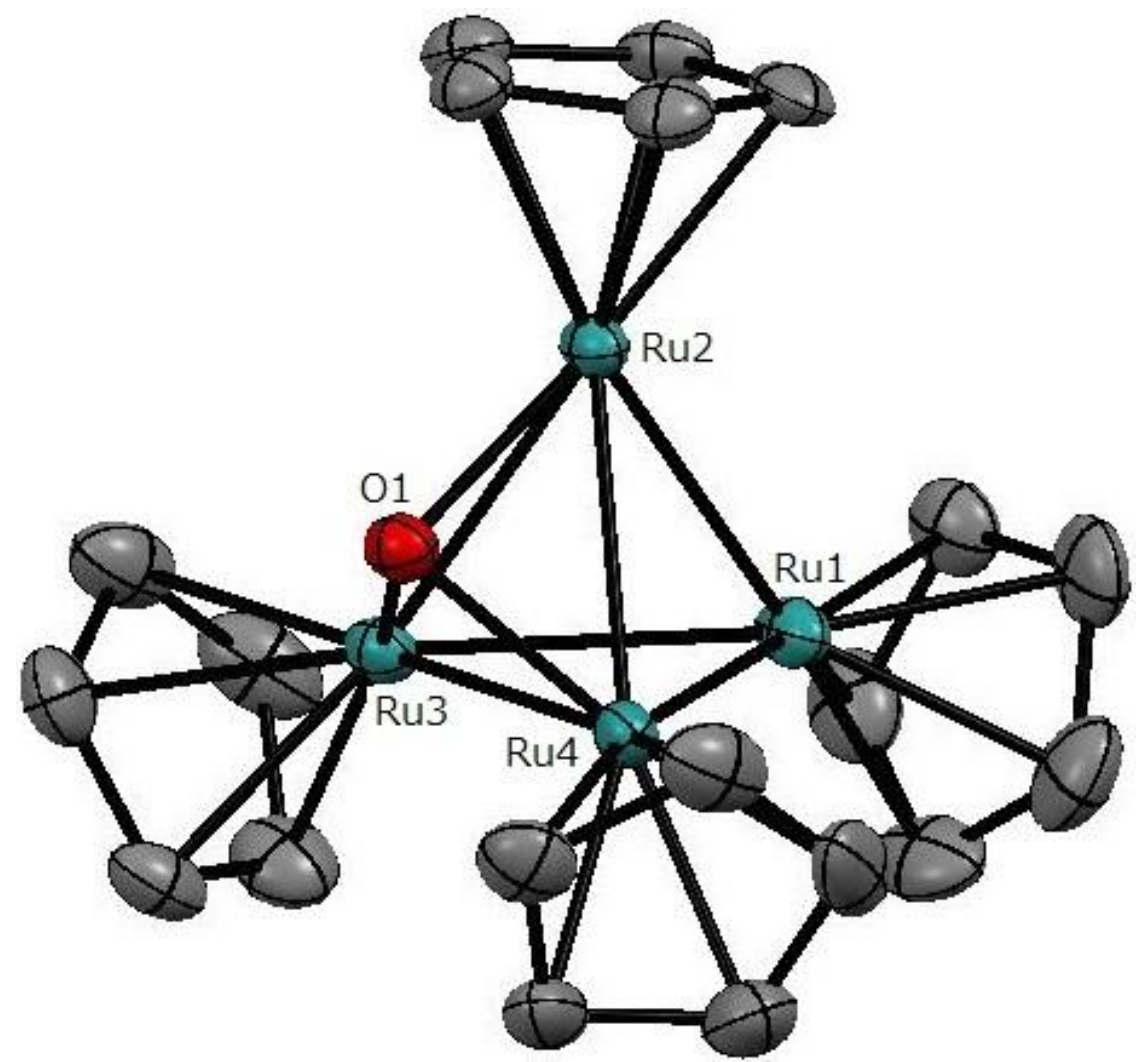

Figure S6 Molecular Structure of $\mathbf{4 b}$ 
Table S5. Crystal data and structure refinement for $\mathbf{4 b}$.

Identification code

Empirical formula

Formula weight

Temperature

Wavelength

Crystal system

Space group

Unit cell dimensions

Volume

$\mathrm{Z}$

Density (calculated)

Absorption coefficient

$\mathrm{F}(000)$

Crystal size

Theta range for data collection

Index ranges

Reflections collected

Independent reflections

Completeness to theta $=25.242^{\circ}$

Refinement method

Data / restraints / parameters

Goodness-of-fit on $\mathrm{F}^{2}$

Final R indices [I>2sigma(I)]

$\mathrm{R}$ indices (all data)

Extinction coefficient

Largest diff. peak and hole
$4 \mathrm{~b}$

C20 H24 O Ru4

684.67

193(2) K

$0.71069 \AA$

Orthorhombic

$P 2{ }_{1} 2_{1} 2_{1}$

$$
\begin{array}{ll}
\mathrm{a}=14.2453(5) \AA & \alpha=90^{\circ} . \\
\mathrm{b}=14.6323(4) \AA & \beta=90^{\circ} . \\
\mathrm{c}=9.3541(3) \AA & \gamma=90^{\circ} .
\end{array}
$$

1949.78(11) $\AA^{3}$

4

$2.332 \mathrm{Mg} / \mathrm{m}^{3}$

$3.044 \mathrm{~mm}^{-1}$

1312

$0.120 \times 0.080 \times 0.050 \mathrm{~mm}^{3}$

3.130 to $25.344^{\circ}$.

$-17<=\mathrm{h}<=17,-17<=\mathrm{k}<=17,-11<=1<=11$

14375

$3556[\mathrm{R}(\mathrm{int})=0.0220]$

$99.9 \%$

Full-matrix least-squares on $\mathrm{F}^{2}$

$3556 / 0$ / 229

1.071

$\mathrm{R} 1=0.0187, \mathrm{wR} 2=0.0467$

$\mathrm{R} 1=0.0192, \mathrm{wR} 2=0.0470$

$\mathrm{n} / \mathrm{a}$

1.038 and -0.408 e. $\AA^{-3}$ 
X-ray Diffraction Analysis on 5

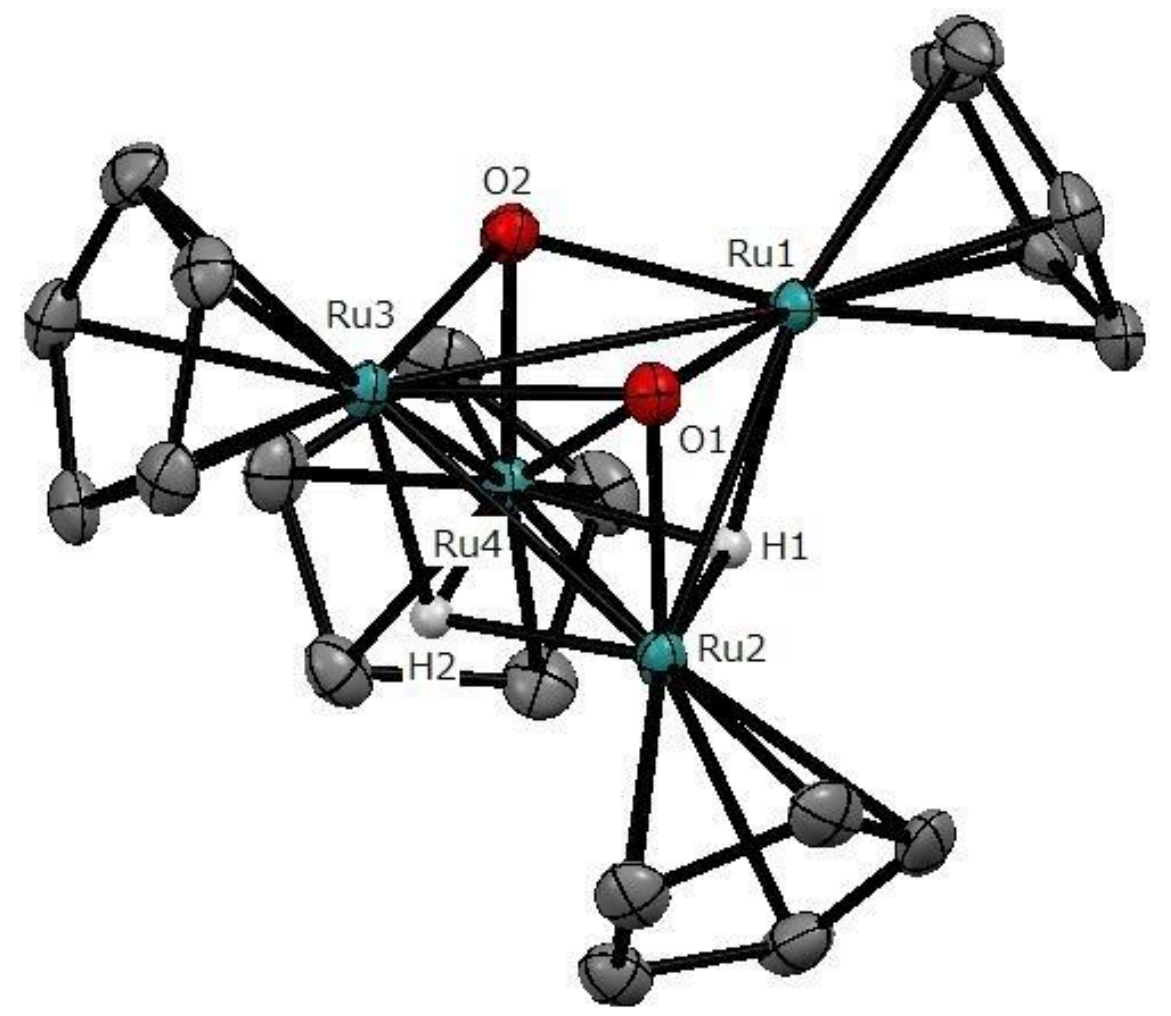

Figure S7 Molecular Structure of 5 
Table S6. Crystal data and structure refinement for $\mathbf{5}$.

Identification code

Empirical formula

Formula weight

Temperature

Wavelength

Crystal system

Space group

Unit cell dimensions

Volume

$\mathrm{Z}$

Density (calculated)

Absorption coefficient

$\mathrm{F}(000)$

Crystal size

Theta range for data collection

Index ranges

Reflections collected

Independent reflections

Completeness to theta $=25.242^{\circ}$

Refinement method

Data / restraints / parameters

Goodness-of-fit on $\mathrm{F}^{2}$

Final R indices [I>2sigma(I)]

$\mathrm{R}$ indices (all data)

Extinction coefficient

Largest diff. peak and hole
5

C20 H24 O3 Ru4

716.67

173(2) K

$0.71069 \AA$

Monoclinic

$P 2{ }_{1} / c$

$\begin{array}{ll}\mathrm{a}=13.9116(4) \AA & \alpha=90^{\circ} . \\ \mathrm{b}=10.6413(3) \AA & \beta=116.3810(13)^{\circ} . \\ \mathrm{c}=14.6536(5) \AA & \gamma=90^{\circ} .\end{array}$

1943.37(10) $\AA^{3}$

4

$2.449 \mathrm{Mg} / \mathrm{m}^{3}$

$3.067 \mathrm{~mm}^{-1}$

1376

$0.180 \times 0.080 \times 0.020 \mathrm{~mm}^{3}$

3.103 to $27.476^{\circ}$.

$-18<=\mathrm{h}<=18,-13<=\mathrm{k}<=13,-18<=\mathrm{l}<=19$

18580

$4428[\mathrm{R}($ int $)=0.0188]$

$99.5 \%$

Full-matrix least-squares on $\mathrm{F}^{2}$

4428 / 0 / 260

1.026

$\mathrm{R} 1=0.0181, \mathrm{wR} 2=0.0424$

$\mathrm{R} 1=0.0198, \mathrm{wR} 2=0.0432$

$\mathrm{n} / \mathrm{a}$

1.220 and -0.475 e. $\AA^{-3}$ 


\section{DFT calculation optimized and X-ray structures}

The structures of tetraruthenium polyhydrido cluster complexes strongly depend on number and locations of hydrido ligands. ${ }^{1}$ The optimized structures of $\mathbf{1 b}, \mathbf{3 a}, \mathbf{3 b}, \mathbf{4 b}$, and $\mathbf{5}$ (Figure S8) reproduce the corresponding X-ray structures well (Table S7), supporting number and locations of hydrido ligands.

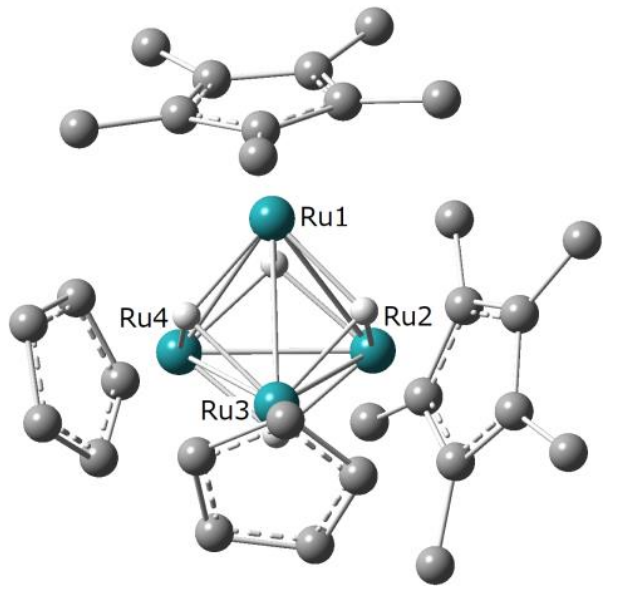

$1 \mathbf{b}$

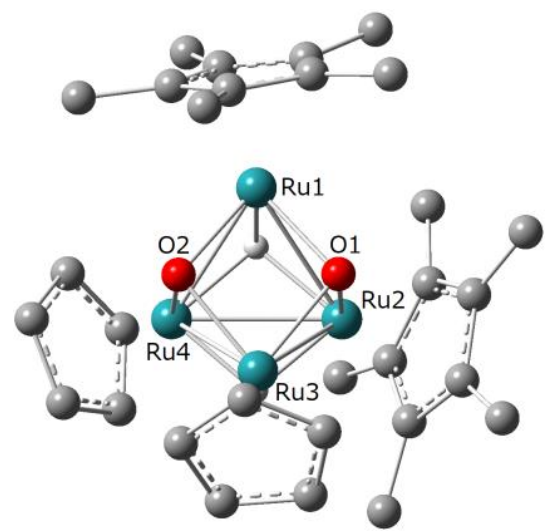

3a
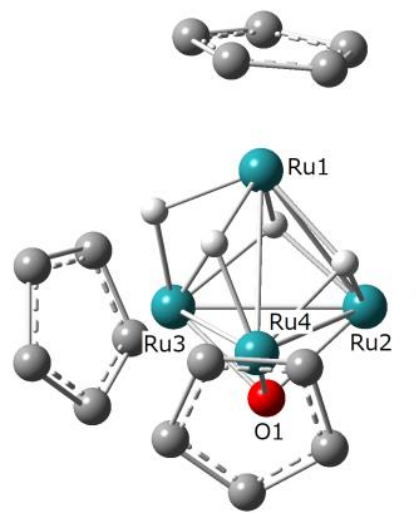

4b

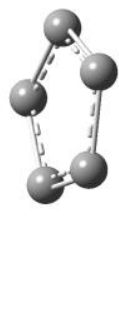

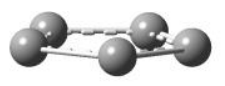

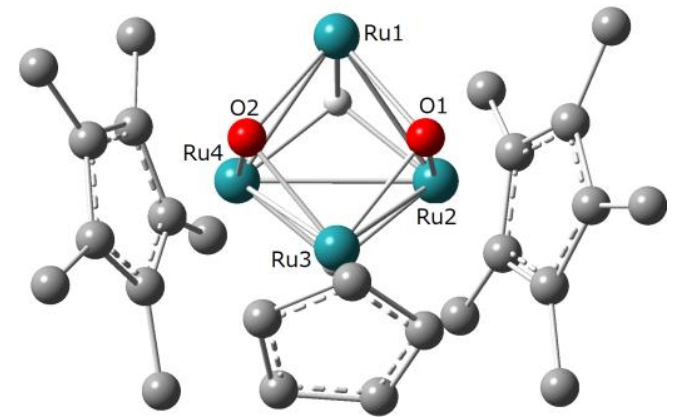

3b

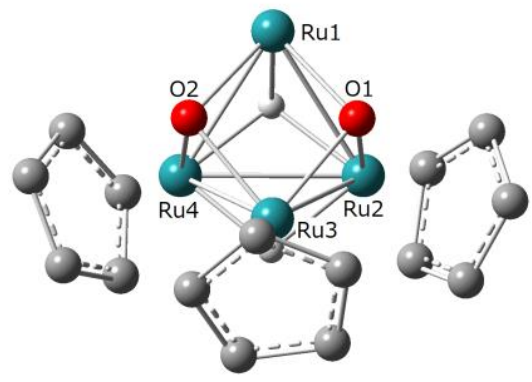

5

Figure S8 Optimized structures of $\mathbf{1 b}, \mathbf{3 a}, \mathbf{3 b}, \mathbf{4 b}, \mathbf{5}$. 
Table S7 Comparison between optimized geometry and X-ray structure

\begin{tabular}{|c|c|c|c|c|c|c|}
\hline & \multicolumn{2}{|r|}{$1 b$} & \multicolumn{2}{|c|}{$3 \mathbf{a}$} & \multicolumn{2}{|r|}{$3 \mathbf{b}$} \\
\hline & DFT & X-ray & DFT & X-ray & DFT & X-ray \\
\hline Ru1-Ru2 [Å] & 2.784 & $2.7713(4)$ & 2.731 & $2.7637(8)$ & 2.712 & $2.7446(5)$ \\
\hline Ru1-Ru3 [Å] & 2.740 & $2.7343(4)$ & 3.125 & $3.1393(9)$ & 3.146 & $3.1567(5)$ \\
\hline Ru1-Ru4 [̊̊] & 2.746 & $2.7270(4)$ & 2.714 & $2.7121(8)$ & 2.708 & $2.7335(5)$ \\
\hline Ru2-Ru3 [Å] & 2.747 & $2.7359(4)$ & 2.702 & $2.7070(9)$ & 2.703 & $2.7423(6)$ \\
\hline Ru2-Ru4 [§̊] & 2.738 & $2.7457(4)$ & 2.719 & $2.7332(10)$ & 2.742 & $2.7923(5)$ \\
\hline Ru3-Ru4 [§] & 2.739 & $2.7576(4)$ & 2.714 & $2.7161(9)$ & 2.714 & $2.7347(7)$ \\
\hline Ru1-O1 [§] & - & - & 2.037 & $2.020(4)$ & 2.026 & $2.033(3)$ \\
\hline Ru1-O2 [Å] & - & - & 2.016 & $2.048(4)$ & 2.037 & $2.034(3)$ \\
\hline Ru2-O1 [Å] & - & - & 1.969 & $1.972(4)$ & 1.965 & $1.962(3)$ \\
\hline Ru3-O1 [Å] & - & - & 2.045 & $2.024(4)$ & 2.027 & $2.033(3)$ \\
\hline Ru3-O2 [Å] & - & - & 2.018 & $2.049(4)$ & 2.037 & $2.025(3)$ \\
\hline $\mathrm{Ru} 4-\mathrm{O} 2[\AA ̊]$ & - & - & 1.976 & $1.970(4)$ & 1.959 & $1.974(3)$ \\
\hline
\end{tabular}

${ }^{a}$ B3PW91-D3/SDD $(\mathrm{Ru})+6-311 \mathrm{G}^{* *}($ Hydrides $)+6-311 \mathrm{G}^{*}(\mathrm{O})+6-31 \mathrm{G}^{*}(\mathrm{C}, \mathrm{H}$ except Hydrides $)$

Table S7 (Cont.)

\begin{tabular}{llllll}
\hline & \multicolumn{3}{c}{$\mathbf{4 b}$} & & \multicolumn{2}{c}{$\mathbf{5}$} \\
\cline { 2 - 3 } \cline { 5 - 6 } \cline { 5 - 6 } Ru1-Ru2 $[\AA]$ & 2.814 & $2.8031(5)$ & & 2.713 & $2.6993(3)$ \\
$\mathrm{Ru} 1-\mathrm{R} u 3[\AA]$ & 2.809 & $2.7973(5)$ & & 3.139 & $3.1483(3)$ \\
$\mathrm{Ru} 1-\mathrm{Ru} 4[\AA]$ & 2.810 & $2.7973(5)$ & & 2.723 & $2.6966(3)$ \\
$\mathrm{Ru} 2-\mathrm{Ru} 3[\AA]$ & 2.796 & $2.7826(5)$ & & 2.720 & $2.7241(3)$ \\
$\mathrm{Ru} 2-\mathrm{Ru} 4[\AA]$ & 2.795 & $2.7845(5)$ & & 2.744 & $2.7507(3)$ \\
$\mathrm{Ru} 3-\mathrm{Ru} 4[\AA]$ & 2.799 & $2.7646(5)$ & & 2.723 & $2.7194(3)$ \\
$\mathrm{Ru} 1-\mathrm{O} 1[\AA]$ & - & - & & 2.020 & $2.0397(16)$ \\
$\mathrm{Ru} 1-\mathrm{O} 2[\AA]$ & - & - & & 2.032 & $2.0391(16)$ \\
$\mathrm{Ru} 2-\mathrm{O} 1[\AA]$ & 1.985 & $1.995(3)$ & & 1.978 & $1.9877(16)$ \\
$\mathrm{Ru} 3-\mathrm{O} 1[\AA]$ & 1.981 & $1.994(3)$ & & 2.026 & $2.0519(16)$ \\
$\mathrm{Ru} 3-\mathrm{O} 2[\AA]$ & - & - & & 2.025 & $2.0483(16)$ \\
$\mathrm{Ru} 4-\mathrm{O} 1[\AA]$ & 1.978 & $1.986(3)$ & & & \\
$\mathrm{Ru} 4-\mathrm{O} 2[\AA]$ & - & - & & 1.972 & $1.9849(16)$ \\
\hline
\end{tabular}

${ }^{a} \mathrm{~B} 3 \mathrm{PW} 91 / \mathrm{SDD}(\mathrm{Ru})+6-311 \mathrm{G}^{* *}($ Hydrides $)+6-311 \mathrm{G}^{*}(\mathrm{O})+6-31 \mathrm{G}^{*}(\mathrm{C}, \mathrm{H}$ except Hydrides $)$ 

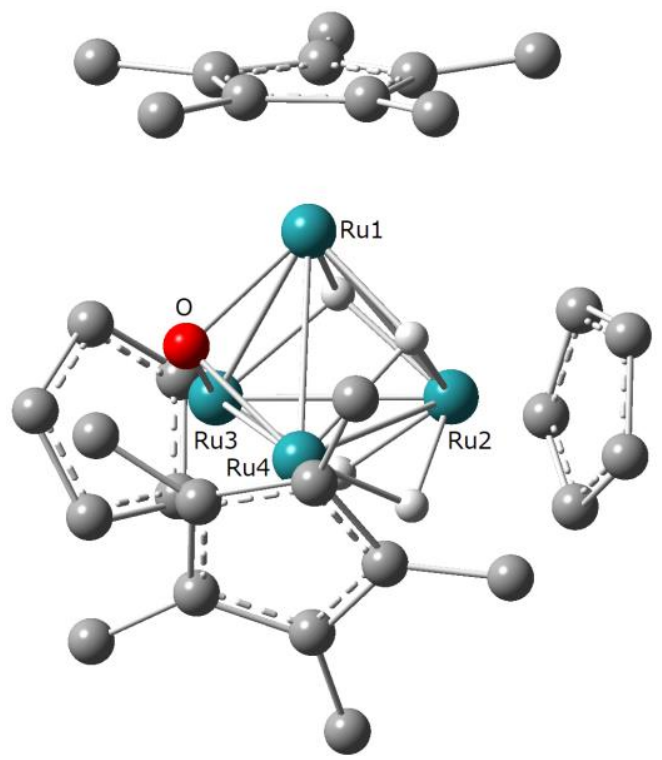

Figure S9. Optimized geometry of $4 a$
Table S8. $\mathrm{Ru}-\mathrm{Ru}$ distances $[\AA ̊ \mathrm{~A}]$ in the optimized geometry of $\mathbf{4 a}$.

\begin{tabular}{ll}
\hline $\mathrm{Ru} 1-\mathrm{Ru} 2$ & 2.812 \\
$\mathrm{Ru} 1-\mathrm{Ru} 3$ & 2.766 \\
$\mathrm{Ru} 1-\mathrm{Ru} 4$ & 2.807 \\
$\mathrm{Ru} 2-\mathrm{Ru} 3$ & 2.804 \\
$\mathrm{Ru} 2-\mathrm{Ru} 4$ & 2.784 \\
$\mathrm{Ru} 3-\mathrm{Ru} 4$ & 2.769 \\
\hline
\end{tabular}

In the crystal of $\mathbf{4 a}$, the $\mu_{3}$-oxo ligand shows disorder over two positions (its ratio was calculated to be 67:33), and therefore the theoretical geometry of 4a was calculated by the DFT method [B3PW91/SDD (Ru),6-311G**(Hydrides),6-311G*(O),6-31G*(C, H except Hydrides)]. Ru-Ru bond distances in the optimized geometry range from 2.766 to $2.812 \AA$ (Table S8), suggesting that $\mathrm{Ru}-\mathrm{Ru}$ bonds in the X-ray structure (2.7910(6)-2.8385(9) $\AA$ ) are somewhat averaged due to the disorder. 


\section{DFT Study on site exchange of hydrido ligands in mono- $\mu_{3}-0 x 0$ complex 4}

${ }^{1} \mathrm{H}$ NMR spectra of mono- $\mu_{3}$-oxo complexes $4 \mathbf{a}$ and $\mathbf{4 b}$ exhibited one equivalent signal for the four hydrides, suggesting the site exchange between $\mu_{3}$-hydride and $\mu$-hydride (for details, see the manuscript). Hence, DFT calculations were performed on the $\mathrm{H}$ site-exchange of $(\mathrm{CpRu})_{4}\left(\mu_{3}-\mathrm{O}\right)\left(\mu_{3}-\mathrm{H}\right)_{2}(\mu-\mathrm{H})_{2}(4 \mathbf{b})$. Figure S10 displayed a plausible mechanism, in which $\mu$-hydride $\mathrm{H}^{\mathrm{a}}$ and $\mu_{3}$-hydride $\mathrm{H}^{\mathrm{b}}$ are simultaneously changed to $\mu_{3}$ - and one $\mu$-hydride, respectively. Its low activation barrier $(1.9 \mathrm{kcal} / \mathrm{mol})$ is consistent with ${ }^{1} \mathrm{H}$ NMR analyses on 4.

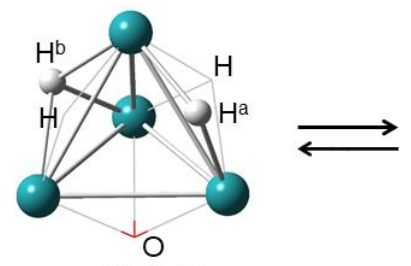

4b (0.0)

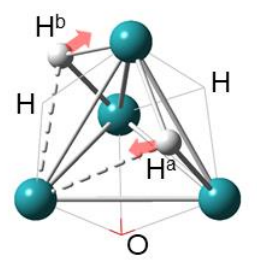

$\mathrm{TS}_{4 \mathrm{~b}}(1.9)$

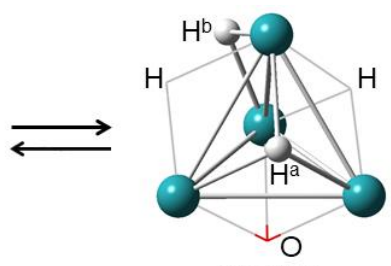

4b (0.0)

Figure S10 Site exchange of hydrido ligands ( $\mathrm{Cp}$ ligands are omitted for clarity). B3PW91-D3/SDD $(\mathrm{Ru}), 6-311+\mathrm{G} * *(\mathrm{H}), 6-311+\mathrm{G} *(\mathrm{C}, \mathrm{O}) / / \mathrm{B} 3 \mathrm{PW} 91 / \mathrm{SDD}(\mathrm{Ru}), 6-311 \mathrm{G} * *$ (Hydrid es),6-311G*(O),6-31G*(C, H except Hydrides $)$. 


\section{DFT Study on Relative Basicity of the Hydrido Ligands in $\mathrm{Cp}_{4}$ and $\mathrm{Cp}_{2}{ }_{2} \mathrm{Cp}_{2}$ systems}

Relative basicity of the hydrido ligands in $\mathbf{2 b}$ and $\mathbf{2 c}$ was evaluated through thermodynamics of $\mathrm{H}^{-}$ transfer between hexahydride 2 and anionic pentahydride $\left[(\mathrm{Cp} * \mathrm{Ru})_{(4-n)}(\mathrm{CpRu})_{\mathrm{n}}(\mathrm{H})_{5}\right]^{-}(\mathbf{6 a}: \mathrm{n}=2, \mathbf{6 b}: \mathrm{n}=4)$ (Figure S11). The reaction is moderately exergonic $(-5.0 \mathrm{kcal} / \mathrm{mol})$, indicating that the replacement of $\mathrm{Cp}$ with $\mathrm{Cp}^{*}$ enhances the basicity of hydrides. This is supported by NBO charges on the hydrides (Table S9). Higher basicity potentially inhibits the $\mathrm{O}-\mathrm{H}$ bond formation.

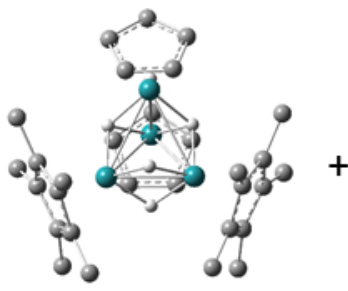

$\left[(\mathrm{CpRu})_{2}\left(\mathrm{Cp}{ }^{\star} \mathrm{Ru}\right)_{2}(\mathrm{H})_{5}\right]^{-}(\mathbf{6 a})$

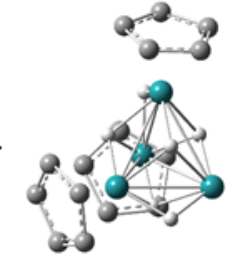

$(\mathrm{CpRu})_{4}(\mathrm{H})_{6}(\mathbf{2 c})$

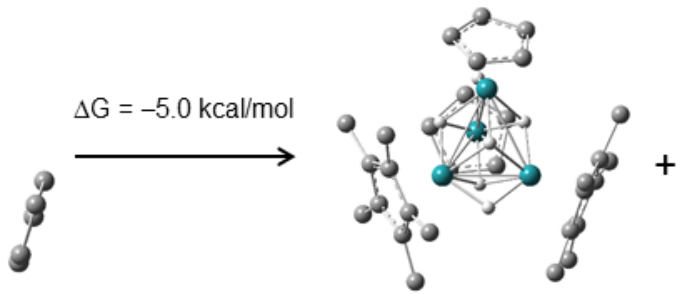

$(\mathrm{CpRu})_{2}\left(\mathrm{Cp}{ }^{\star} \mathrm{Ru}\right)_{2}(\mathrm{H})_{6}(\mathbf{2 b})$

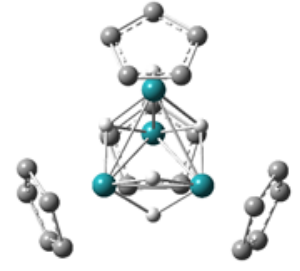

$\left[(\mathrm{CpRu})_{4}(\mathrm{H})_{5}\right]^{-}(\mathbf{6 b})$

Figure S11 Hydride transfer between anionic pentahydrido complex $\left[(\mathrm{Cp} * \mathrm{Ru})_{2}(\mathrm{CpRu})_{2}(\mathrm{H})_{5}\right]^{-}(\mathbf{6 a})$ and neutral hexahydrido complex $(\mathrm{CpRu})_{4}(\mathrm{H})_{6} \quad(\mathbf{2 c})$. B3PW91-D3/SDD $(\mathrm{Ru}), 6-311+\mathrm{G}^{* *}(\mathrm{H}), 6-311+\mathrm{G}^{*}(\mathrm{C}, \mathrm{O})$ //B3PW91-D3/SDD $(\mathrm{Ru}), 6-311 \mathrm{G} * *($ Hydrides $), 6-311 \mathrm{G} *(\mathrm{O}), 6-31 \mathrm{G} *(\mathrm{C}, \mathrm{H}$ except Hydrides $)$.

Table S9 NBO charges on hydrides in neutral hexahydrido 2 and anionic pentahydrido 6 complexes

\begin{tabular}{lllll}
\hline & $(\mathrm{Cp} * \mathrm{Ru})_{2}(\mathrm{CpRu})_{2}(\mathrm{H})_{6}(\mathbf{2 b})$ & $(\mathrm{CpRu})_{4}(\mathrm{H})_{6}(\mathbf{2 c})$ & {$\left[(\mathrm{Cp} * \mathrm{Ru})_{2}(\mathrm{CpRu})_{2}(\mathrm{H})_{5}\right]^{-}(\mathbf{6 a})$} & {$\left[(\mathrm{CpRu})_{4}(\mathrm{H})_{5}\right]^{-}(\mathbf{6 b})$} \\
\hline$\mu_{3}-\mathrm{H}$ & 0.2193 & 0.2322 & 0.1929 & 0.2057 \\
$\mu_{3}-\mathrm{H}$ & 0.2200 & 0.2333 & 0.1931 & 0.2052 \\
$\mu_{3}-\mathrm{H}$ & 0.2233 & 0.2322 & - & - \\
$\mu_{3}-\mathrm{H}$ & 0.2228 & 0.2332 & - & - \\
$\mu-\mathrm{H}$ & 0.2273 & 0.2240 & 0.1740 & 0.1938 \\
$\mu-\mathrm{H}$ & 0.2020 & 0.2239 & 0.1717 & 0.1930 \\
$\mu-\mathrm{H}$ & - & - & 0.1829 & 0.1789 \\
\hline
\end{tabular}




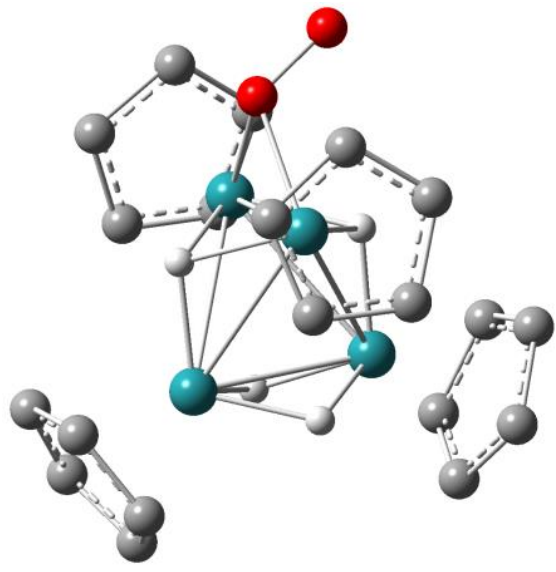

$\mu-\eta^{1}: \eta^{1}\left({ }^{\perp}\right)$ coordination, singlet, $16.3 \mathrm{kcal} / \mathrm{mol}$
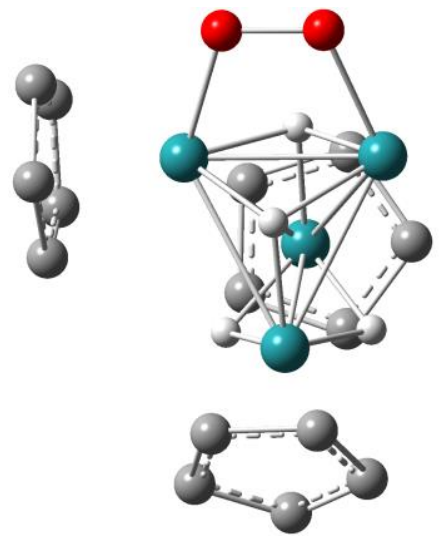

$\mu-\eta^{1}: \eta^{1}(\|)$ coordination, singlet, $15.4 \mathrm{kcal} / \mathrm{mol}$
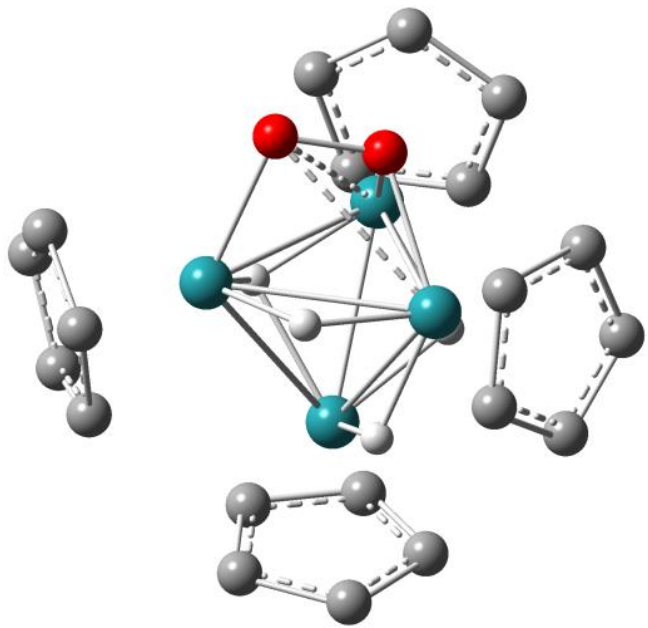

$\mu_{3-} \eta^{1}: \eta^{2}: \eta^{2}$ coordination, singlet (A1), $0.0 \mathrm{kcal} / \mathrm{mol}$

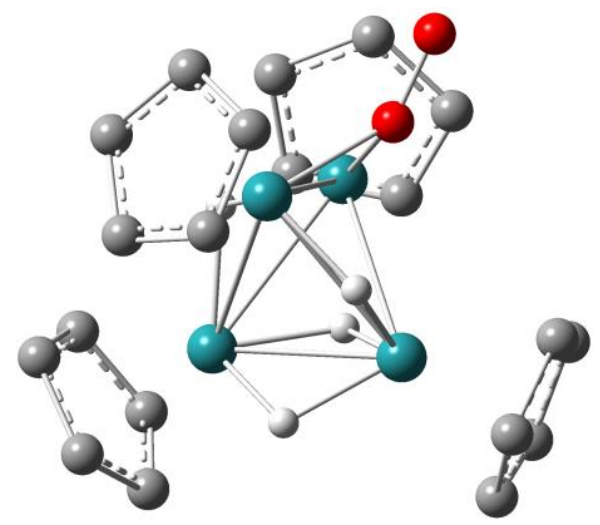

$\mu-\eta^{1}: \eta^{1}(\perp)$ coordination, triplet, $19.6 \mathrm{kcal} / \mathrm{mol}$
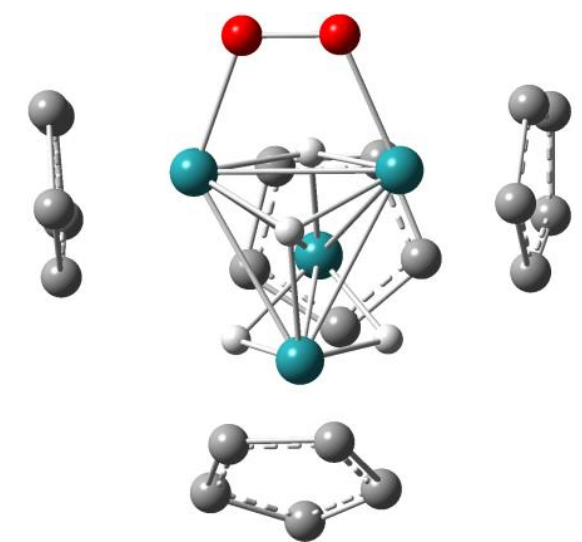

$\mu-\eta^{1}: \eta^{1}(\|)$ coordination, triplet, $21.3 \mathrm{kcal} / \mathrm{mol}$

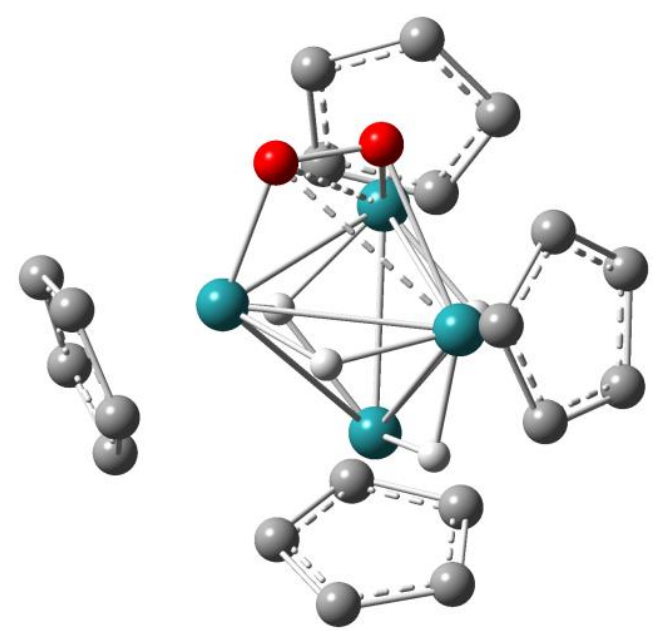

$\mu_{3}-\eta^{1}: \eta^{2}: \eta^{2}$ coordination, triplet, $13.5 \mathrm{kcal} / \mathrm{mol}$

Figure S12 Coordination modes of $\mathrm{O}_{2}$, spin state, and Gibbs free energy relative to that of $\mathbf{A 1}$ (Sea-green:

$\mathrm{Ru}$, Red: O, Gray: C, White: H). B3PW91-D3/SDD (Ru),6-311+G**(H),6-311+G*(C,O) //B3PW91/SDD(Ru),6-311G**(Hydrides),6-311G*(O),6-31G*(C, H except Hydrides). 
DFT calculations on $\mathrm{O}_{2}$ adduct of $(\mathrm{CpRu})_{4} \mathrm{H}_{6}(2 \mathrm{c})$
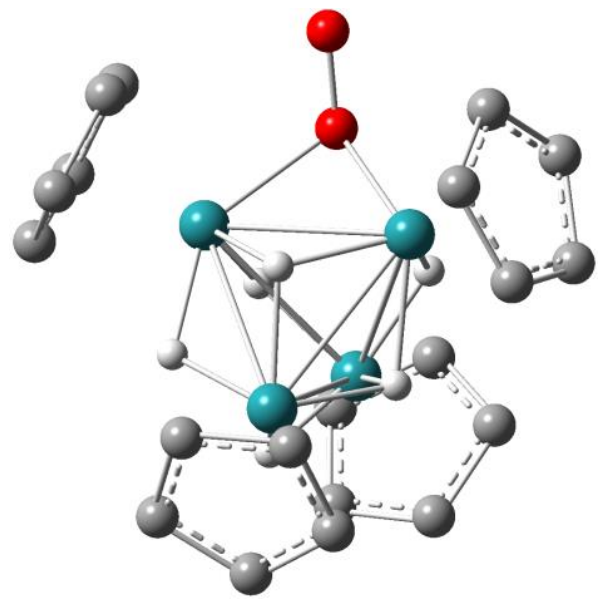

$\mu-\eta^{1}: \eta^{1}(\perp)$ coordination, singlet, $-1.2 \mathrm{kcal} / \mathrm{mol}$

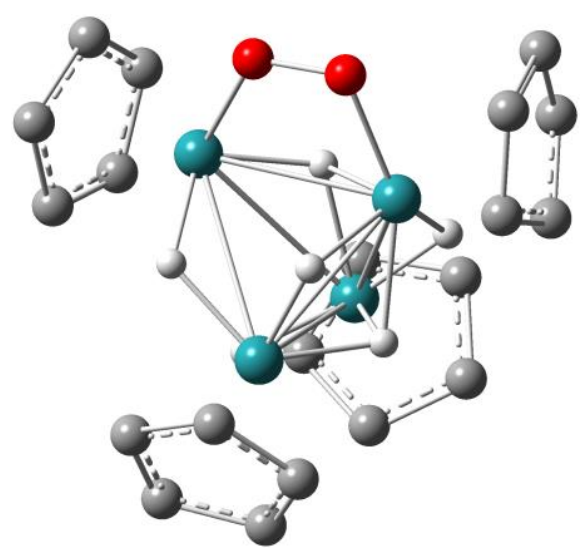

$\mu-\eta^{1}: \eta^{1}(\|)$ coordination, singlet, $23.3 \mathrm{kcal} / \mathrm{mol}$

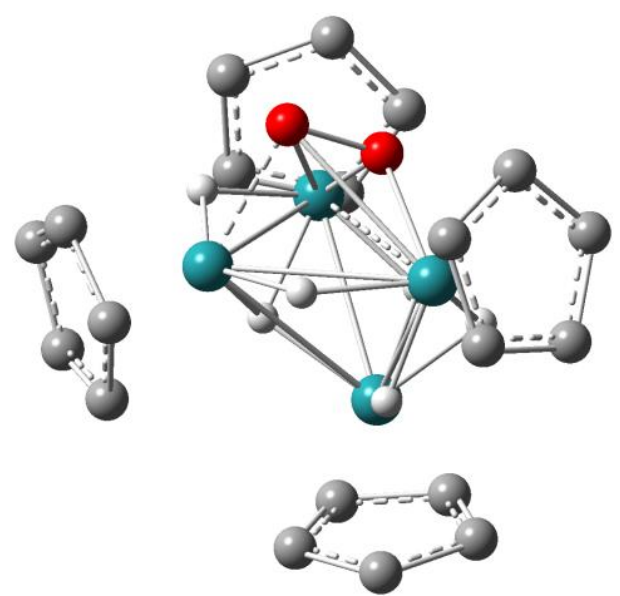

$\mu_{3}-\eta^{1}: \eta^{2}: \eta^{2}$ coordination, singlet (B1), $0.0 \mathrm{kcal} / \mathrm{mol}$

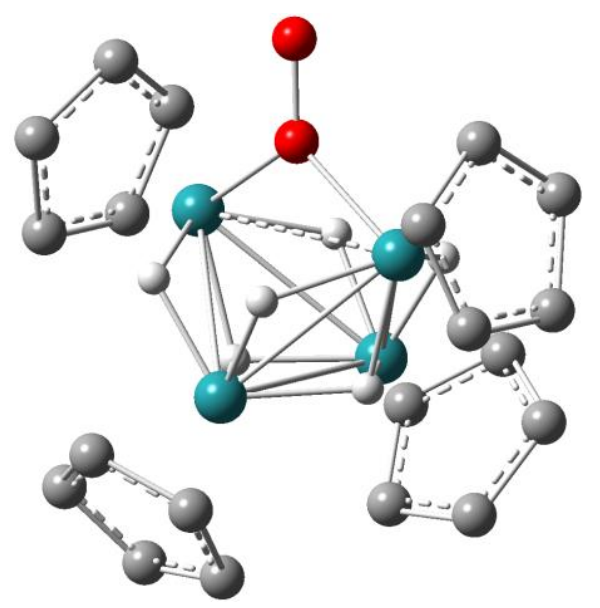

$\mu-\eta^{1}: \eta^{1}\left({ }^{\perp}\right)$ coordination, triplet, $-5.1 \mathrm{kcal} / \mathrm{mol}$
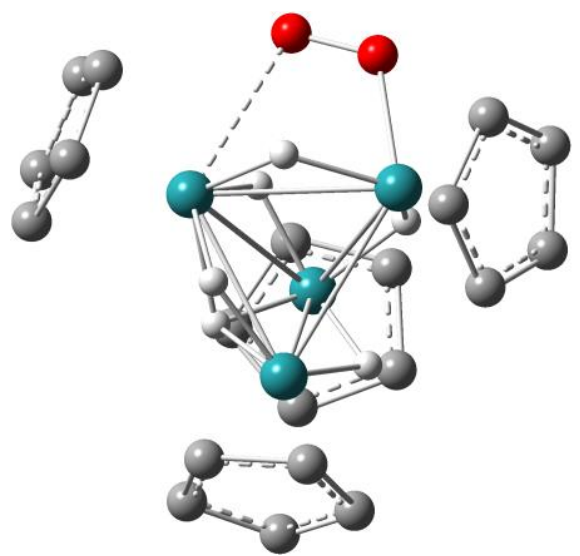

$\mu-\eta^{1}: \eta^{1}(\|)$ coordination, triplet, $7.0 \mathrm{kcal} / \mathrm{mol}$

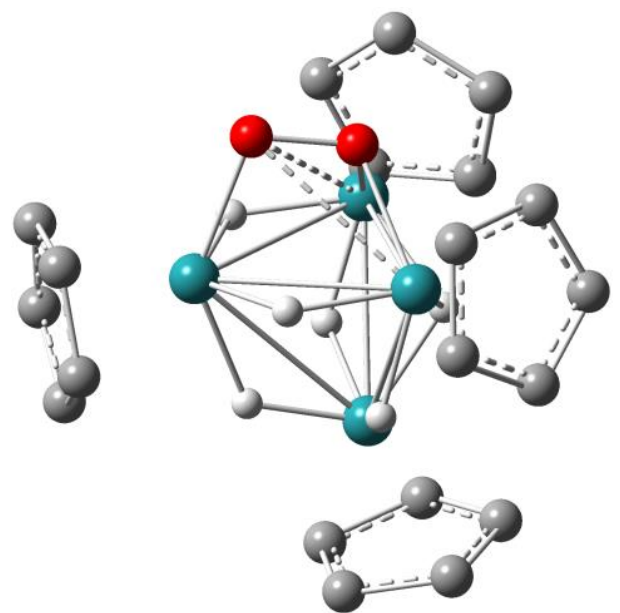

$\mu_{3}-\eta^{1}: \eta^{2}: \eta^{2}$ coordination, triplet, $12.2 \mathrm{kcal} / \mathrm{mol}$

Figure S13 Coordination modes of $\mathrm{O}_{2}$, spin state, and Gibbs free energy relative to that of $\mathbf{B} \mathbf{1}$ (Sea-green:

$\mathrm{Ru}, \quad$ Red: O, Gray: C, White: H). B3PW91-D3/SDD $(\mathrm{Ru}), 6-311+\mathrm{G}^{* *}(\mathrm{H}), 6-311+\mathrm{G}^{*}(\mathrm{C}, \mathrm{O})$ //B3PW91/SDD(Ru),6-311G**(Hydrides),6-311G*(O),6-31G*(C, H except Hydrides). 
DFT Calculations on Oxygenation Reactions of $(\mathrm{CpRu})_{4} \mathrm{H}_{4}(1 \mathrm{c})$

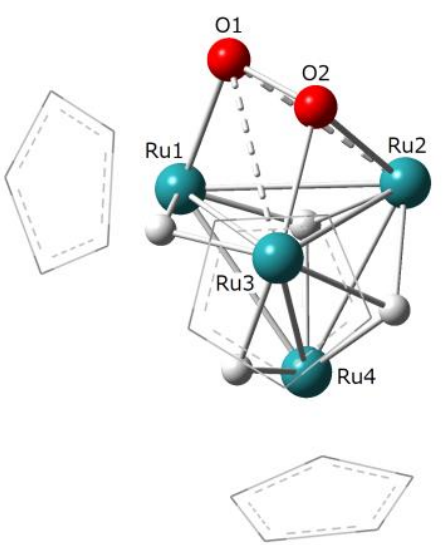

A1
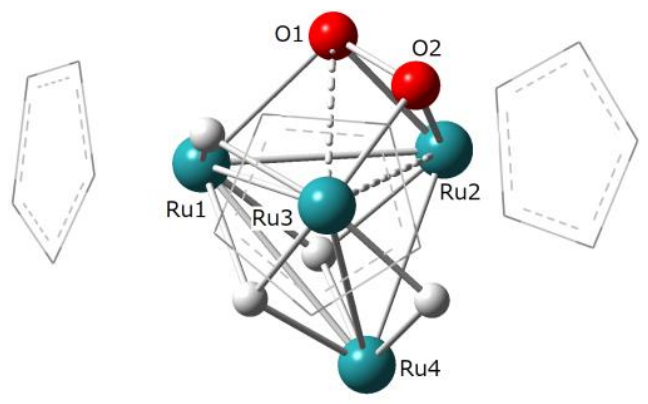

A2
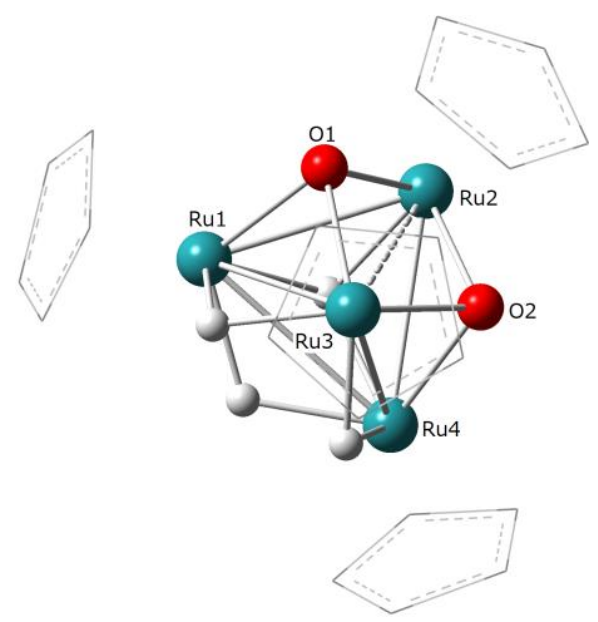

A3

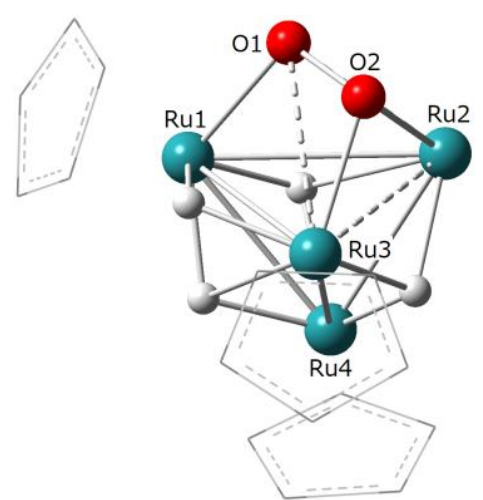

$\mathbf{T S}_{\mathrm{A} \mathbf{1} / \mathrm{A} 2}(\mathrm{Ru}-\mathrm{Ru}$ Cleavage $)$
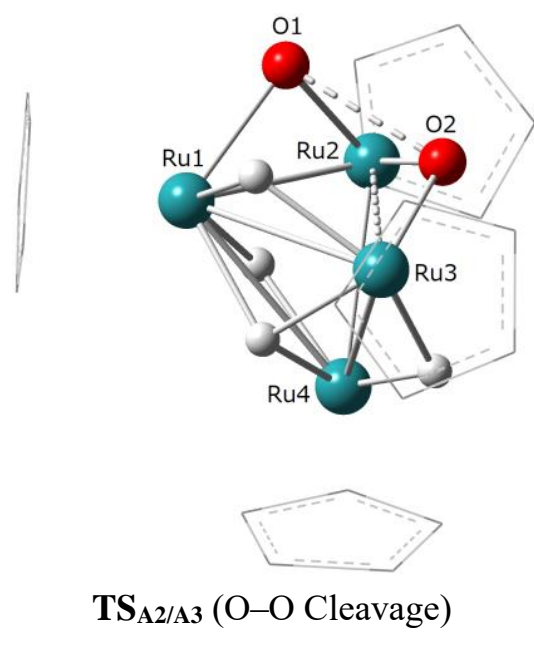

$\mathbf{T S}_{\mathrm{A} 2 / \mathrm{A} 3}(\mathrm{O}-\mathrm{O}$ Cleavage $)$
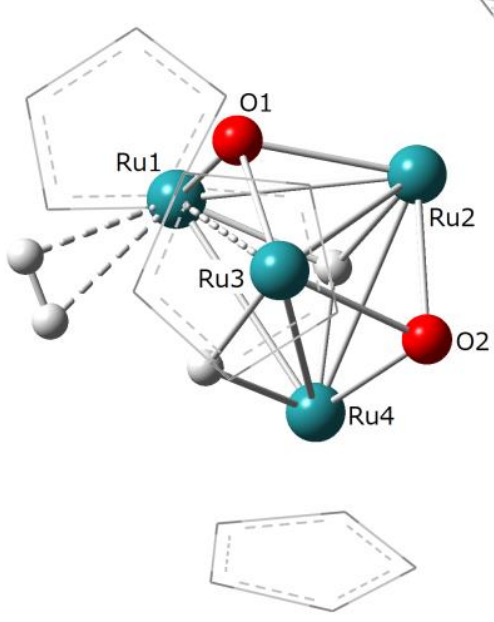

$\mathbf{T S}_{\mathrm{A} 3 / \mathrm{A} 4}(\mathrm{H}-\mathrm{H}$ Formation $)$

Figure S14 Optimized structures concerning the oxygenation of 1c (B3PW91/SDD(Ru)+ 6-311G**(Hydrides)+6-311G*(O)+6-31G*(C, H except Hydrides $)$ ). 

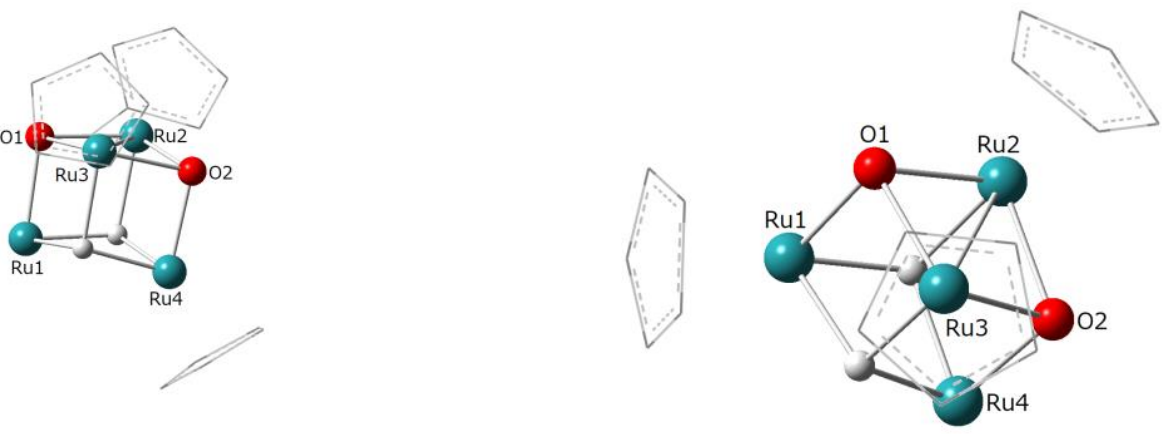

${ }_{\mathrm{H}}^{\mathrm{H}} \mathrm{O}$

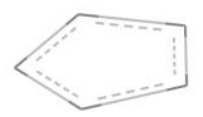

$\mathrm{A} 4+\mathrm{H}_{2}$

A4
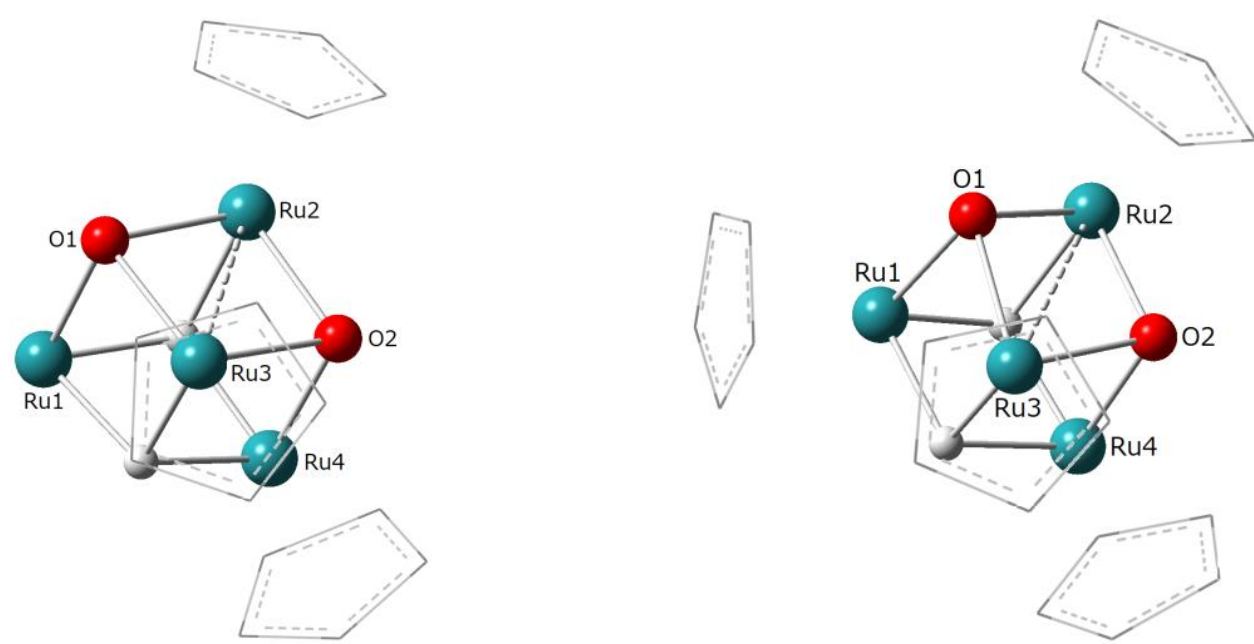

$\mathbf{T S}_{\mathrm{A} 4 / 5}(\mathrm{Ru}-\mathrm{Ru}$ Cleavage $)$

5
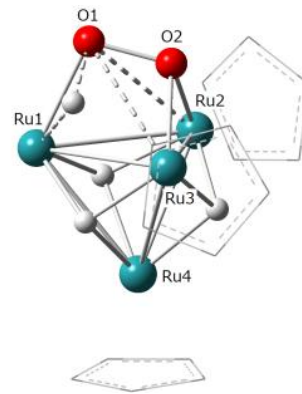

TS $_{\text {A1/A5 }}(\mathrm{O}-\mathrm{H}$ Formation)

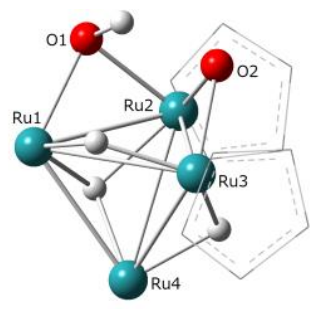

A5
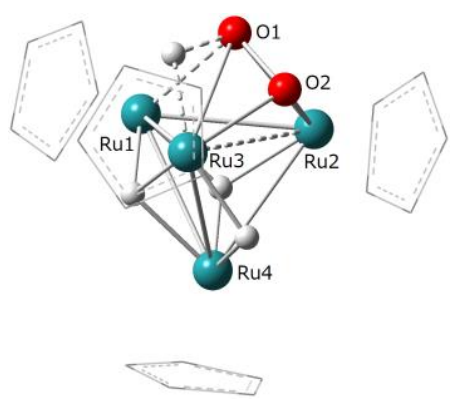

TS $_{\text {A2/A5 }}(\mathrm{O}-\mathrm{H}$ Formation)

Figure S14 (Cont.) 
Table S10 Bond distance change in the oxygenation of 1c

\begin{tabular}{llllllll} 
& $\mathbf{1 c}$ & $\mathbf{A 1}$ & $\mathbf{T S}_{\mathbf{A} 1 / \mathbf{A} 2}$ & $\mathbf{A 2}$ & $\mathbf{T S}_{\mathbf{A} 2 / \mathbf{A} 3}$ & $\mathbf{A 3}$ & $\mathbf{T S}_{\mathbf{A} 3 / \mathbf{A} 4}$ \\
\hline $\mathrm{Ru} 1-\mathrm{Ru} 2[\AA]$ & 2.727 & 2.869 & 3.179 & 2.828 & 2.742 & 2.799 & 2.978 \\
$\mathrm{Ru} 1-\mathrm{Ru} 3[\AA]$ & 2.728 & 2.845 & 2.878 & 2.853 & 2.817 & 2.792 & 3.489 \\
$\mathrm{Ru} 1-\mathrm{Ru} 4[\AA]$ & 2.728 & 2.797 & 2.817 & 2.935 & 2.947 & 2.935 & 2.934 \\
$\mathrm{Ru} 2-\mathrm{Ru} 3[\AA]$ & 2.723 & 2.736 & 2.760 & 3.538 & 3.420 & 3.239 & 2.580 \\
$\mathrm{Ru} 2-\mathrm{Ru} 4[\AA]$ & 2.726 & 2.717 & 2.721 & 2.784 & 2.707 & 2.799 & 2.865 \\
$\mathrm{Ru} 3-\mathrm{Ru} 4[\AA]$ & 2.728 & 2.837 & 2.879 & 2.826 & 2.831 & 2.794 & 2.788 \\
$\mathrm{Ru} 1-\mathrm{O} 1[\AA]$ & - & 2.067 & 2.117 & 2.062 & 1.968 & 2.004 & 2.054 \\
$\mathrm{Ru} 2-\mathrm{O} 1[\AA]$ & - & 2.806 & 2.478 & 2.027 & 1.943 & 2.044 & 2.040 \\
$\mathrm{Ru} 2-\mathrm{O} 2[\AA]$ & - & 2.027 & 2.024 & 2.113 & 2.094 & 2.046 & 2.008 \\
$\mathrm{Ru} 3-\mathrm{O} 1[\AA]$ & - & 2.873 & 2.858 & 2.951 & 3.228 & 2.052 & 2.033 \\
$\mathrm{Ru} 3-\mathrm{O} 2[\AA]$ & - & 2.052 & 2.041 & 2.100 & 1.897 & 2.053 & 2.010 \\
$\mathrm{Ru} 4-\mathrm{O} 2[\AA]$ & - & - & - & 3.302 & 3.065 & 2.003 & 1.974 \\
$\mathrm{O} 1-\mathrm{O} 2[\AA]$ & - & 1.416 & 1.434 & 1.460 & 2.220 & 2.461 & - \\
\hline
\end{tabular}

${ }^{a} \mathrm{~B} 3 \mathrm{PW} 91 / \mathrm{SDD}(\mathrm{Ru})+6-311 \mathrm{G}^{* *}($ Hydrides $)+6-311 \mathrm{G}^{*}(\mathrm{O})+6-31 \mathrm{G}^{*}(\mathrm{C}$, H except Hydrides $)$

Table S10 (Cont.)

\begin{tabular}{|c|c|c|c|c|c|c|}
\hline & $\mathrm{A} 4+\mathrm{H}_{2}$ & A4 & $\mathbf{T S}_{\mathrm{A} 4 / 5}$ & $\mathbf{T S}_{\mathrm{A} 1 / \mathrm{A} 5}$ & A5 & $\mathbf{T S}_{\mathrm{A} 2 / \mathrm{A} 5}$ \\
\hline Ru1-Ru2 [Å] & 2.908 & 2.913 & 2.855 & 2.909 & 2.770 & 2.837 \\
\hline Ru1-Ru3 [§̊] & 2.923 & 2.916 & 2.836 & 2.830 & 2.996 & 2.885 \\
\hline $\mathrm{Ru} 1-\mathrm{Ru} 4[\AA]$ & 2.884 & 2.887 & 2.821 & 2.829 & 2.722 & 2.885 \\
\hline Ru2-Ru3 [Å] & 2.623 & 2.621 & 2.855 & 2.759 & 2.733 & 3.585 \\
\hline Ru2-Ru4 [Å] & 2.923 & 2.920 & 2.830 & 2.725 & 2.999 & 2.971 \\
\hline Ru3-Ru4 [§̊] & 2.912 & 2.917 & 2.855 & 2.842 & 2.703 & 2.771 \\
\hline Ru1-O1 [§̊] & 1.969 & 1.969 & 1.969 & 2.147 & 2.037 & 2.192 \\
\hline Ru2-O1 [A] & 2.019 & 2.017 & 2.073 & 2.867 & 2.075 & 2.287 \\
\hline $\mathrm{Ru} 2-\mathrm{O} 2[\AA]$ & 2.019 & 2.020 & 1.997 & 2.041 & 1.986 & 2.083 \\
\hline Ru3-O1 [A] & 2.020 & 2.018 & 1.995 & 2.776 & 1.964 & 2.776 \\
\hline Ru3-O2 [Å] & 2.016 & 2.019 & 2.070 & 2.067 & 2.053 & 2.124 \\
\hline $\mathrm{Ru} 4-\mathrm{O} 2[\AA]$ & 1.970 & 1.970 & 1.973 & - & - & - \\
\hline $\mathrm{O} 1-\mathrm{O} 2[\AA \AA]$ & - & - & - & 1.467 & 2.410 & 1.494 \\
\hline
\end{tabular}

${ }^{a} \mathrm{~B} 3 \mathrm{PW} 91 / \mathrm{SDD}(\mathrm{Ru})+6-311 \mathrm{G} * *($ Hydrides $)+6-311 \mathrm{G}^{*}(\mathrm{O})+6-31 \mathrm{G}^{*}(\mathrm{C}, \mathrm{H}$ except Hydrides $)$ 
DFT Calculations on Oxygenation Reactions of $(\mathrm{CpRu})_{4} \mathrm{H}_{6}(2 \mathrm{c})$
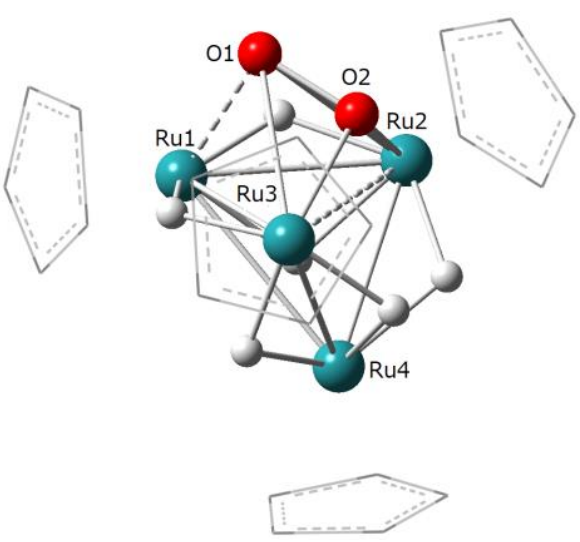

B1

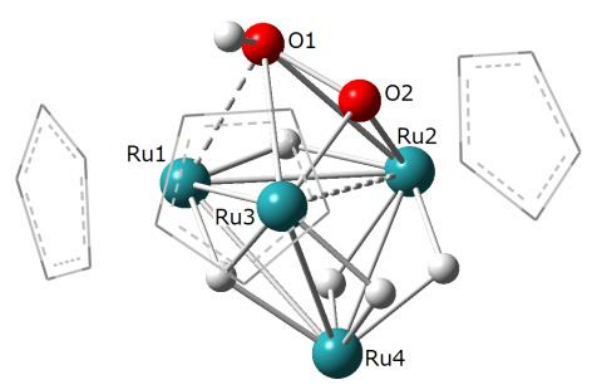

B2

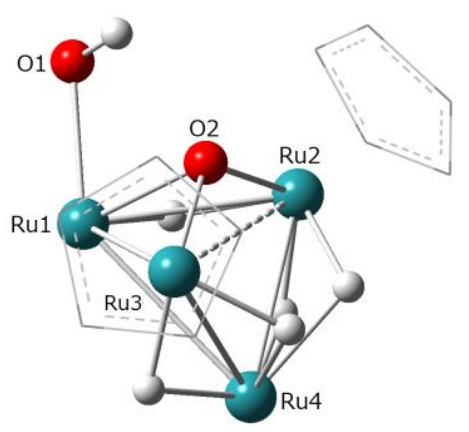

B3

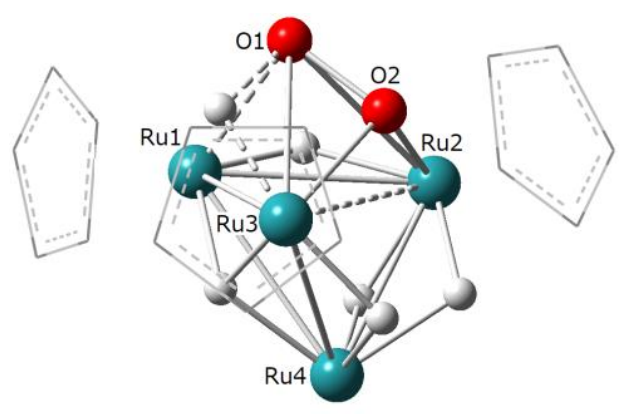

$\mathbf{T S}_{\mathbf{B} 1 / \mathbf{B} 2}(\mathrm{O}-\mathrm{H}$ Formation)
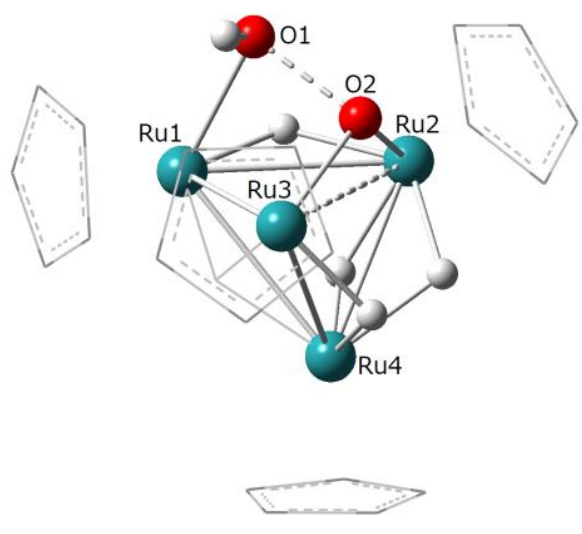

$\mathbf{T S}_{\mathbf{B} 2 / \mathbf{B} 3}(\mathrm{O}-\mathrm{O}$ Cleavage)

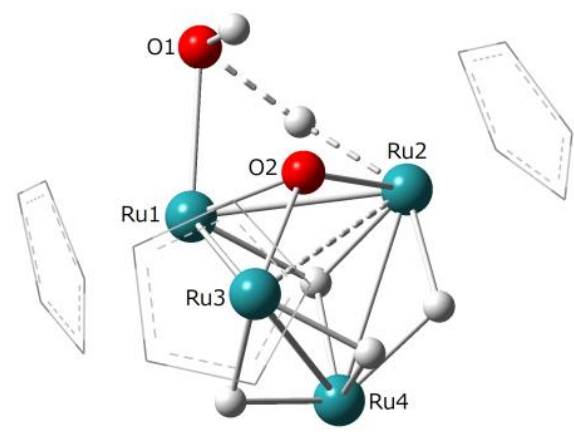

$\mathbf{T S}_{\mathbf{B} 3 / \mathbf{B} 4}(\mathrm{O}-\mathrm{H}$ Formation)

Figure S15 Optimized structures concerning the oxygenation of 2c (B3PW91/SDD $(\mathrm{Ru})+$ 6-311G**(Hydrides $)+6-311 G^{*}(\mathrm{O})+6-31 \mathrm{G} *(\mathrm{C}, \mathrm{H}$ except Hydrides $)$ ). 

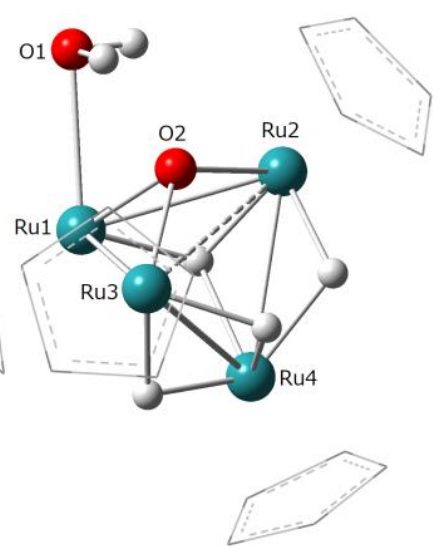

B4
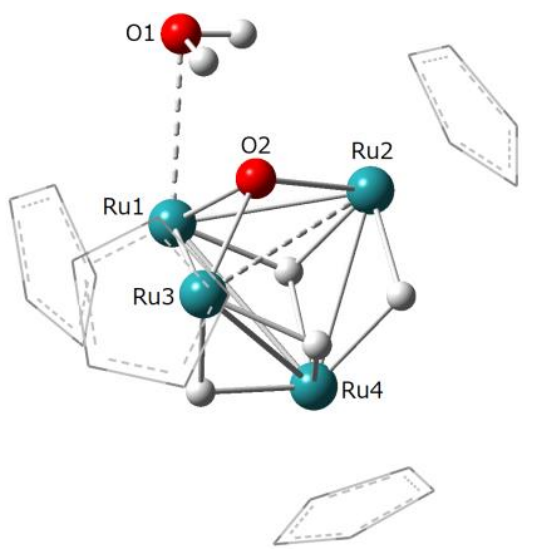

$\mathbf{T S}_{\mathbf{B} 4 / 4 \mathbf{b}}(\mathrm{Ru}-\mathrm{O}$ Cleavage)

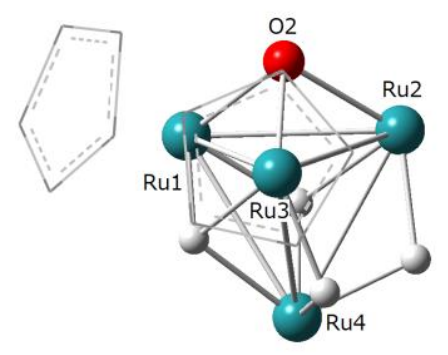

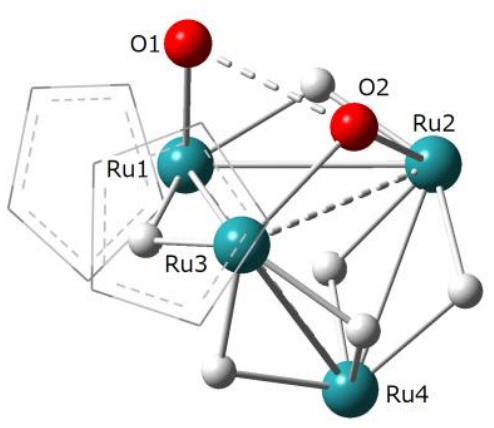

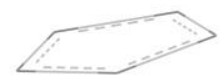

$\mathbf{T S}_{\mathbf{B} 2 / \mathbf{B 5}}$ (O-O Cleavage)

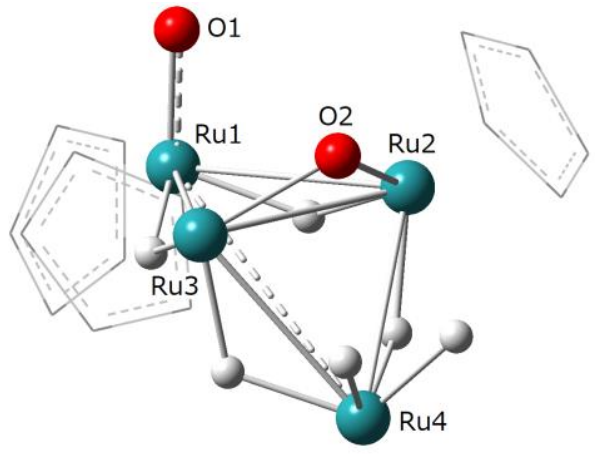

B5

Figure S15 (Cont.) 
Table S11 Bond distance change in the oxygenation of $\mathbf{2 c}$

\begin{tabular}{lllllll} 
& $\mathbf{2 c}$ & $\mathbf{B 1}$ & $\mathbf{T S}_{\mathbf{B} 1 / \mathbf{B} 2}$ & $\mathbf{B 2}$ & $\mathbf{T S}_{\mathbf{B} 2 / \mathbf{B 3}}$ & $\mathbf{B 3}$ \\
\hline $\mathrm{Ru} 1-\mathrm{Ru} 2[\AA]$ & 2.680 & 2.865 & 2.921 & 2.909 & 2.928 & 2.769 \\
$\mathrm{Ru} 1-\mathrm{Ru} 3[\AA]$ & 2.926 & 2.965 & 2.863 & 2.997 & 2.916 & 2.772 \\
$\mathrm{Ru} 1-\mathrm{Ru} 4[\AA]$ & 2.927 & 3.078 & 3.024 & 3.036 & 3.039 & 3.028 \\
$\mathrm{Ru} 2-\mathrm{Ru} 3[\AA]$ & 2.926 & 3.599 & 3.595 & 3.639 & 3.502 & 3.581 \\
$\mathrm{Ru} 2-\mathrm{Ru} 4[\AA]$ & 2.926 & 2.846 & 2.795 & 2.839 & 2.800 & 2.787 \\
$\mathrm{Ru} 3-\mathrm{Ru} 4[\AA]$ & 2.680 & 2.762 & 2.805 & 2.754 & 2.772 & 2.758 \\
$\mathrm{Ru} 1-\mathrm{O} 1[\AA]$ & - & 2.080 & 2.151 & 2.224 & 2.179 & 2.074 \\
$\mathrm{Ru} 1-\mathrm{O} 2[\AA]$ & - & - & - & 2.797 & 2.786 & 2.001 \\
$\mathrm{Ru} 2-\mathrm{O} 1[\AA]$ & - & 2.752 & 2.825 & 2.855 & 2.924 & 3.263 \\
$\mathrm{Ru} 2-\mathrm{O} 2[\AA]$ & - & 2.133 & 2.122 & 2.147 & 2.091 & 2.071 \\
$\mathrm{Ru} 3-\mathrm{O} 1[\AA]$ & - & 2.752 & 2.702 & 2.767 & 2.860 & 4.106 \\
$\mathrm{Ru} 3-\mathrm{O} 2[\AA]$ & - & 2.122 & 2.150 & 2.176 & 2.006 & 1.996 \\
$\mathrm{O} 1-\mathrm{O} 2[\AA]$ & - & 1.446 & 1.474 & 1.493 & 1.766 & 2.559 \\
\hline
\end{tabular}

${ }^{a} \mathrm{~B} 3 \mathrm{PW} 91 / \mathrm{SDD}(\mathrm{Ru})+6-311 \mathrm{G}^{* *}($ Hydrides $)+6-311 \mathrm{G}^{*}(\mathrm{O})+6-31 \mathrm{G}^{*}(\mathrm{C}, \mathrm{H}$ except Hydrides $)$

Table S11 (Cont.)

\begin{tabular}{|c|c|c|c|c|c|c|}
\hline & $\mathbf{T S}_{\mathbf{B} 3 / \mathbf{B} 4}$ & B4 & $\mathbf{T S}_{\mathbf{B} 4 / 4 \mathrm{~b}}$ & $4 \mathrm{~b}+\mathrm{H}_{2} \mathrm{O}$ & $\mathbf{T S}_{\mathbf{B} 1 / \mathbf{B} 5}$ & B5 \\
\hline Ru1-Ru2 [Å] & 2.740 & 2.752 & 2.719 & 2.819 & 2.845 & 3.140 \\
\hline Ru1-Ru3 [§̊] & 2.784 & 2.818 & 2.799 & 2.816 & 2.979 & 3.129 \\
\hline $\mathrm{Ru} 1-\mathrm{Ru} 4[\AA]]$ & 2.967 & 2.969 & 2.971 & 2.820 & 3.298 & 4.466 \\
\hline Ru2-Ru3 [Å] & 3.623 & 3.631 & 3.626 & 2.815 & 3.424 & 2.983 \\
\hline Ru2-Ru4 [Å] & 2.836 & 2.792 & 2.784 & 2.803 & 2.798 & 2.967 \\
\hline Ru3-Ru4 [̊̊] & 2.765 & 2.784 & 2.792 & 2.810 & 2.759 & 2.964 \\
\hline Ru1-O1 [§̊] & 2.140 & 2.367 & 2.556 & - & 1.863 & 1.763 \\
\hline Ru1-O2 [A] & 2.021 & 2.020 & 2.007 & 1.988 & 2.847 & 3.648 \\
\hline Ru2-O1 [Å] & - & - & - & - & - & - \\
\hline Ru2-O2 [A] & 2.109 & 2.109 & 2.104 & 1.986 & 2.022 & 1.887 \\
\hline Ru3-O1 [Å] & - & - & - & - & - & - \\
\hline Ru3-O2 [Å] & 1.995 & 2.005 & 2.008 & 1.983 & 1.987 & 1.888 \\
\hline $\mathrm{O} 1-\mathrm{O} 2[\AA \AA]$ & - & - & - & - & 2.266 & 3.419 \\
\hline
\end{tabular}

${ }^{a} \mathrm{~B} 3 \mathrm{PW} 91 / \mathrm{SDD}(\mathrm{Ru})+6-311 \mathrm{G} * *($ Hydrides $)+6-311 \mathrm{G}^{*}(\mathrm{O})+6-31 \mathrm{G}^{*}(\mathrm{C}, \mathrm{H}$ except Hydrides $)$ 


\section{DFT calculations on thermodynamics for dihydrogen elimination from tetrahydrido complex}

The possibility of dihydrogen elimination from tetrahydrido complex was considered here, because its feasibility is strongly related with the reaction mechanism (i.e. associative or dissociative mechanism). The dihydrogen elimination processes were found to be very endergonic ( $\Delta G$ values are more than $42 \mathrm{kcal} / \mathrm{mol}$ ) in both $\mathrm{Cp}_{4}$ and $\mathrm{Cp}_{2} \mathrm{Cp}_{2}$ systems (Scheme $\mathrm{S} 1$ ), supporting the relevance of the associative mechanism for bis-oxygenation of tetrahydrido complex.

(a)

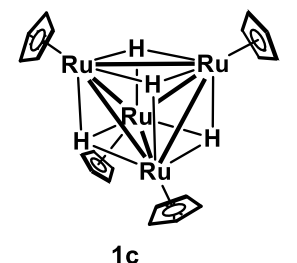

(b)

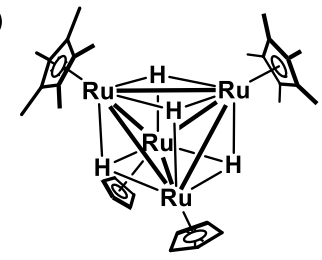

$1 \mathrm{~b}$

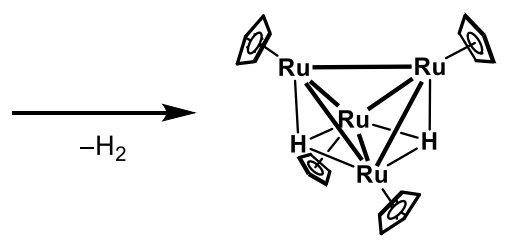

7
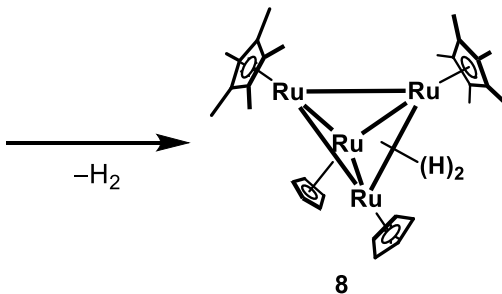

$\Delta \mathrm{G}=42.2 \mathrm{kcal} / \mathrm{mol}$

$\Delta G=44.6 \mathrm{kcal} / \mathrm{mol}($ for $8 \mathrm{a})$

$\Delta \mathrm{G}=44.0 \mathrm{kcal} / \mathrm{mol}($ for $8 \mathrm{~b})$

$\Delta \mathrm{G}=42.4 \mathrm{kcal} / \mathrm{mol}$ (for $8 \mathrm{c}$ )

Scheme S1 Free Gibbs energy changes in dihydrogen elimination from tetrahydrido complexes 1c (a) and 1b (b). The product $\mathbf{8}$ has three positional isomers 8a-8c (as shown in Figure S16) with respect to the relative positions of the $\mathrm{Cp}^{*}$ and $\mathrm{Cp}$ ligands.

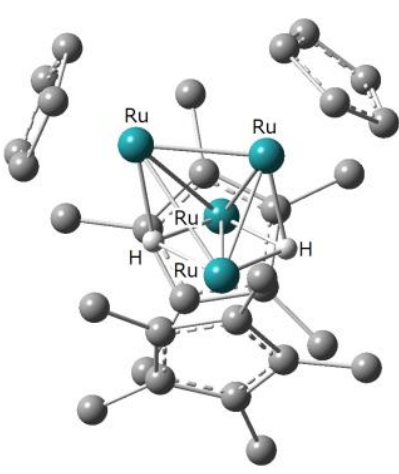

$8 \mathrm{a}$

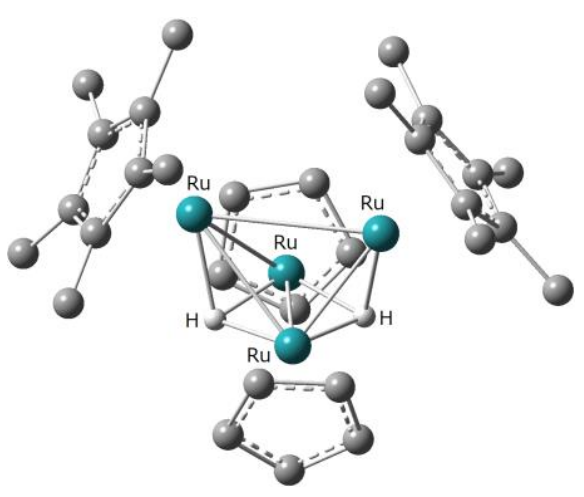

$8 b$

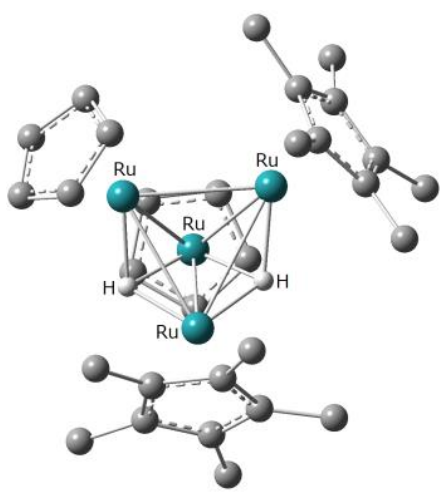

$8 \mathrm{c}$

Figure S16 Optimized structures of dihydrido complexes 8 . 


\section{Orbitals analysis on the fragment $\mathrm{C}\left(\left[(\mathrm{CpRu})_{4} \mathrm{H}_{\mathrm{n}}\right]^{2+}\right)$ in $\mathrm{TS}_{\mathrm{A} 2 / \mathrm{A} 3}$ and $\mathrm{TS}_{\mathrm{B} 1 / \mathrm{B} 5}$}

Figures S17-S18 display frontier orbitals of the fragments $C\left(\left[(\mathrm{CpRu})_{4} \mathrm{H}_{\mathrm{n}}\right]^{2+}\right)$ in $\mathbf{T S}_{\mathbf{A 2} / \mathbf{A 3}}(\mathrm{n}=4)$ and $\mathbf{T S}_{\mathbf{B} \mathbf{B} \mathbf{B 5}}(\mathrm{n}=6)$. The orbital energy levels of $\mathbf{T} \mathbf{S}_{\mathbf{A} 2 / \mathbf{A} \mathbf{3}}$ which are lower than those of $\mathbf{T} \mathbf{S}_{\mathbf{B} \mathbf{B} \mathbf{B} 5}$ are likely to induce relatively stronger $\sigma$ donation from the $\left[\mathrm{O}_{2}\right]^{2-}$ moiety and weaker $\pi$-back donation to the $\left[\mathrm{O}_{2}\right]^{2-}$ moiety.

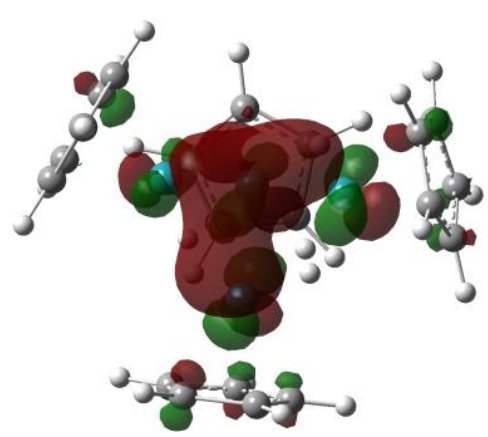

HOMO (-4.31eV)

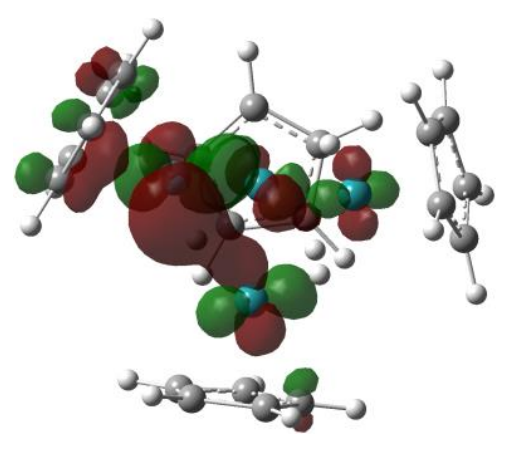

LUMO $(-2.76 \mathrm{eV})$

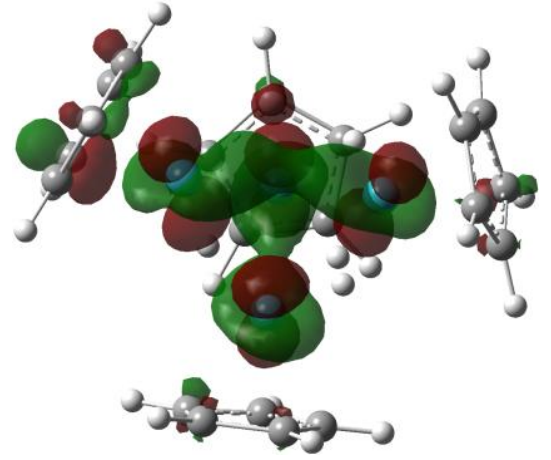

LUMO+1 $(-1.90 \mathrm{eV})$

Figure S17 Frontier orbitals (Isovalue 0.04) of the fragments $\mathrm{C}\left(\left[(\mathrm{CpRu})_{4} \mathrm{H}_{4}\right]^{2+}\right)$ in $\mathbf{T} \mathbf{S}_{\mathbf{A} 2 / \mathbf{A} 3}$

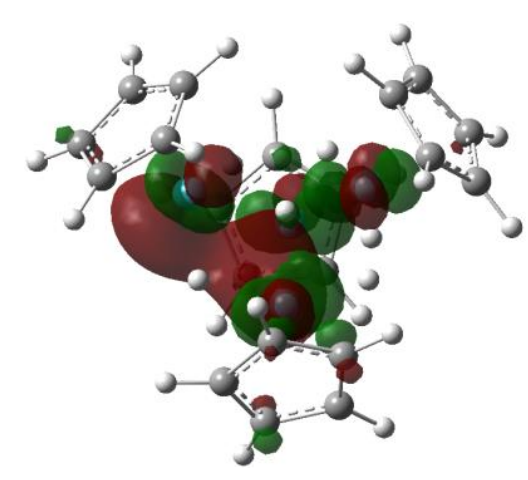

HOMO $(-3.99 \mathrm{eV})$

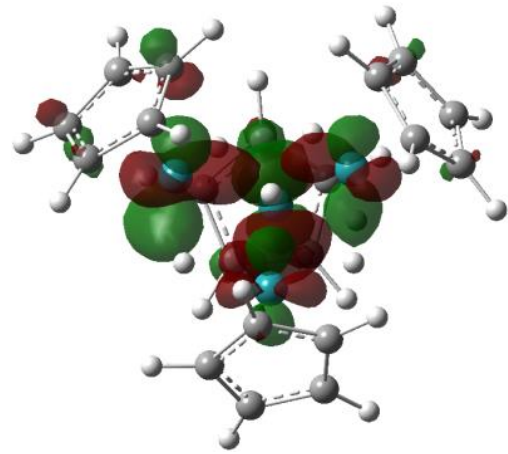

LUMO (-2.06 eV)

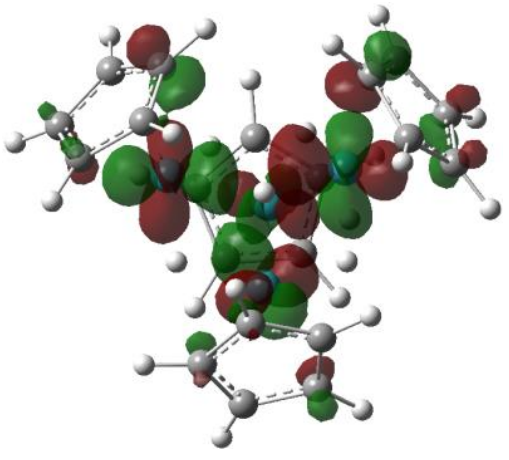

$\mathrm{LUMO}+1(-1.70 \mathrm{eV})$

Figure S18 Frontier orbitals (Isovalue 0.04$)$ of the fragments $\mathrm{C}\left(\left[(\mathrm{CpRu})_{4} \mathrm{H}_{6}\right]^{2+}\right)$ in $\mathbf{T S}_{\mathbf{B} \mathbf{1} / \mathbf{B} \mathbf{5} \text {. }}$ B3PW91-D3/SDD $(\mathrm{Ru}), 6-311+\mathrm{G}^{* *}(\mathrm{H}), 6-311+\mathrm{G}^{*}(\mathrm{C}) / / \mathrm{B} 3 \mathrm{PW} 91 / \mathrm{SDD}(\mathrm{Ru}), 6-311 \mathrm{G}^{* *}$ (Hydrides), 6-311G*(O ),6-31G*(C, H except Hydrides). 


\section{Mechanistic study on the hopping of $\mu_{3}$-oxo ligand on $\mathbf{R u}$ face}

Mechanistic study was performed on the isomerization of bis- $\mu_{3}$-oxo species 3 via the hopping of $\mu_{3}$-oxo ligand using DFT calculations. Three possible pathways were considered here; (i) via the $\mu$-oxo ligand, (ii) via the $\mu$-hydroxo ligand, and (iii) via the $\mu_{4}$-oxo intermediate accompanied with $\mathrm{Ru}-\mathrm{Ru}$ bond cleavage (Scheme S2). Here, $\left[(\mathrm{CpRu})_{4}\left(\mu_{3}-\mathrm{O}\right)_{2}\left(\mu_{3}-\mathrm{H}\right)_{2}\right]$ (5) bearing four Cp ligand was employed to save the cost of calculations (Figure S19). After $\mathrm{Ru}-\mathrm{Ru}$ bond formation affording A4, the pathway (i) occurs with 28.3 $\mathrm{kcal} / \mathrm{mol}$ of Gibbs activation energy ( $\Delta \mathrm{G}^{0 \ddagger}$ ) through the $\mu$-oxo transition state $\mathbf{T S}_{\mathbf{A 4}}$. On the other hand, the pathway (ii) requires significantly high Gibb activation energy $\left(\Delta \mathrm{G}^{0 \ddagger}=39.0 \mathrm{kcal} / \mathrm{mol}\right)$ for the formation of intermediary $\mu$-hydroxo species A6. The pathway (iii) takes place through $\mathbf{T S}_{\mathbf{A} 4 / \mathbf{A} 7}$ with $35.6 \mathrm{kcal} / \mathrm{mol}$ of Gibb activation energy $\left(\Delta \mathrm{G}^{*}\right)$ to afford $\mu_{4}$-oxo intermediate $\mathbf{A 7}$, which is higher than that of (i). Therefore, it is likely that the transfer via $\mu$-oxo ligand (i) is a main pathway for the hopping of the $\mu_{3}$-oxo ligand.

(i) Transfer of $\mu$-oxo ligand

$R u=C p R u$ or $C p * R u$

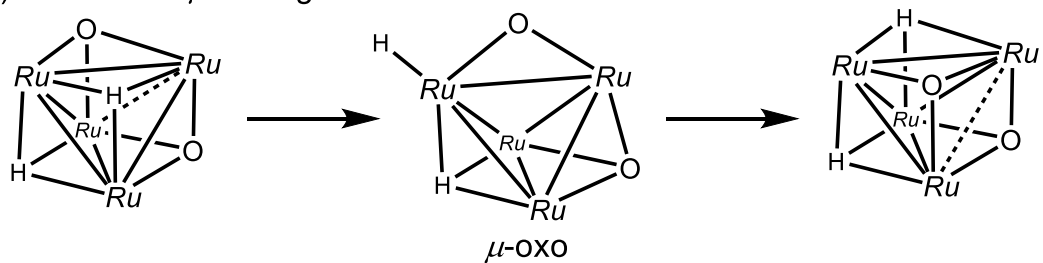

(ii) Transfer of $\mu$-hydroxo ligand

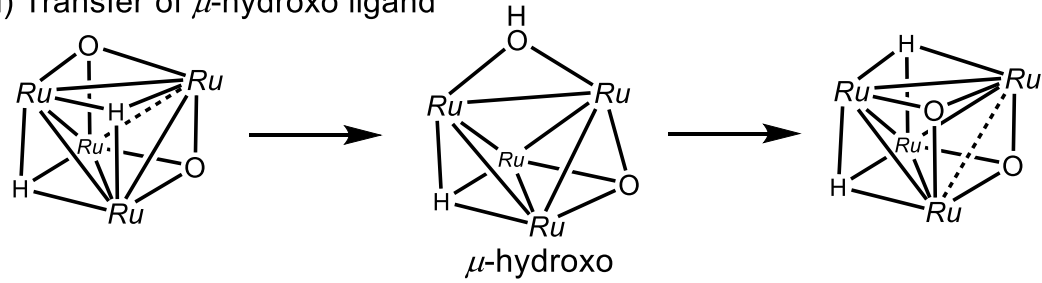

(iii) Formation of $\mu_{4}$-oxo intermediate accompanied with Ru-Ru bond cleavage

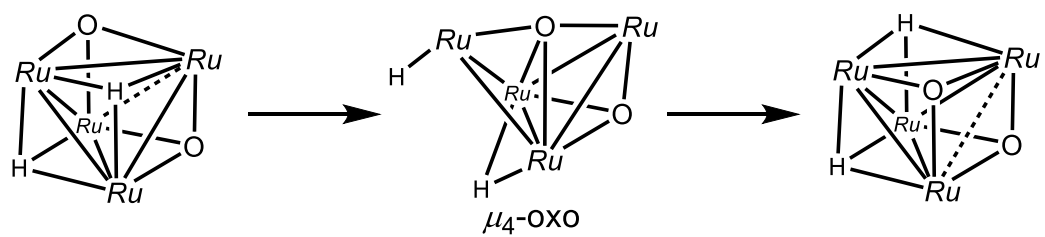

Scheme S2 Possible pathways for the isomerization of bis- $\mu_{3}$-oxo complex 3 . 
(i) Transfer via $\mu$-Oxo Transition State

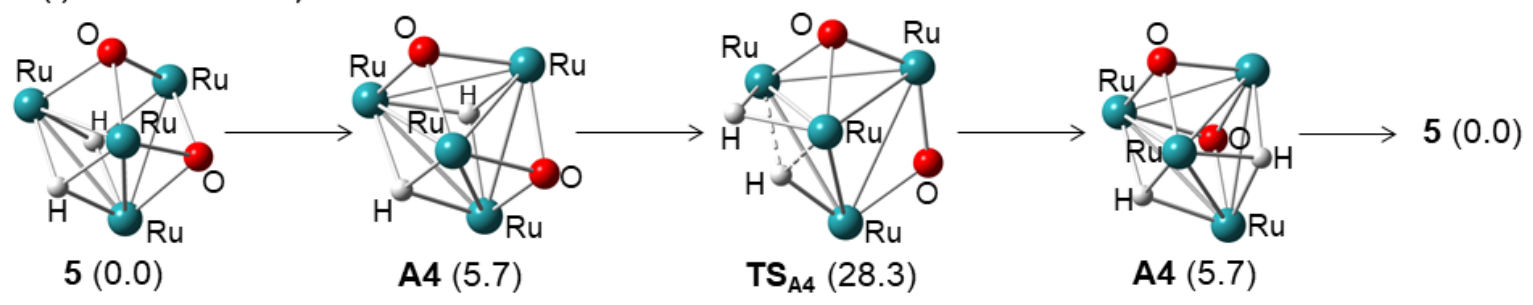

(ii) Transfer via $\mu$-Hydroxo Intermediate

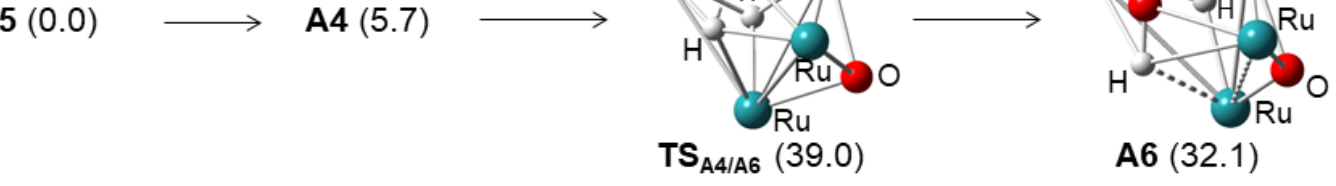

(iii) Transfer via $\mu_{4}$-Oxo Intermediate

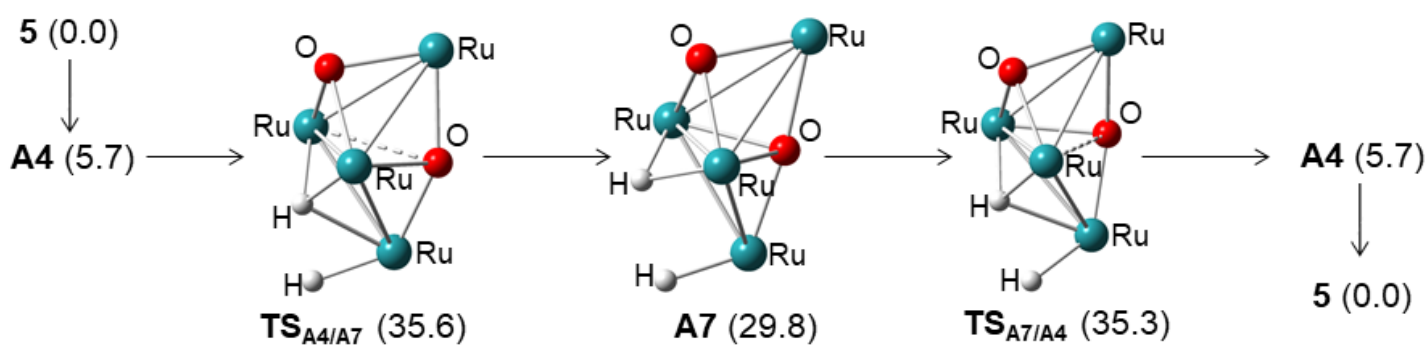

Figure S19 Gibbs energy and geometry changes (at $363.15 \mathrm{~K}$ ) in three isomerization pathways of bis- $\mu_{3}$-oxo complex $\left[(\mathrm{CpRu})_{4}\left(\mu_{3}-\mathrm{O}\right)_{2}\left(\mu_{3}-\mathrm{H}\right)_{2}\right] \quad$ (5). $\mathrm{Cp}$ ligands were omitted for clarity. B3PW91-D3/SDD $(\mathrm{Ru}), 6-311+\mathrm{G}^{* *}(\mathrm{H}), 6-311+\mathrm{G} *(\mathrm{C}, \mathrm{O}) / / \mathrm{B} 3 \mathrm{PW} 91 / \mathrm{SDD}(\mathrm{Ru}), 6-311 \mathrm{G} * *$ (Hydrides), 6-311G*(O),6-31G*(C, H except Hydrides). 


\section{Important Cartesian Coordination}

\section{$4 \mathrm{~b}$}

$\mathrm{E}(\mathrm{RB} 3 \mathrm{PW} 91)=-1231.77424804$ A.U.

Thermal correction to Gibbs Free Energy= 0.319859 A.U.

\begin{tabular}{|c|c|c|c|}
\hline $\mathrm{Ru}$ & 4.22473100 & 2.19401000 & 6.04651100 \\
\hline $\mathrm{Ru}$ & 2.15983700 & 0.51266400 & 5.19521800 \\
\hline $\mathrm{Ru}$ & 2.86743200 & 2.75676200 & 3.65201800 \\
\hline $\mathrm{Ru}$ & 4.59028100 & 0.54251000 & 3.81609500 \\
\hline $\mathrm{O}$ & 4.06114200 & 0.25010800 & 5.69987700 \\
\hline $\mathrm{C}$ & 5.37236600 & 1.94162700 & 7.92266800 \\
\hline $\mathrm{C}$ & 4.10475300 & 2.54015900 & 8.19919800 \\
\hline $\mathrm{C}$ & 4.05547400 & 3.80332500 & 7.51166900 \\
\hline $\mathrm{C}$ & 5.29228300 & 3.96087600 & 6.80990400 \\
\hline $\mathrm{C}$ & 6.10515000 & 2.80778400 & 7.05904800 \\
\hline $\mathrm{C}$ & 1.16852700 & -0.97899900 & 6.47873300 \\
\hline $\mathrm{C}$ & 1.07869800 & -1.38306000 & 5.11018300 \\
\hline $\mathrm{C}$ & 0.33138600 & -0.37206500 & 4.40678900 \\
\hline $\mathrm{C}$ & -0.03899900 & 0.63917300 & 5.34158100 \\
\hline $\mathrm{C}$ & 0.49560800 & 0.27061600 & 6.62170500 \\
\hline $\mathrm{C}$ & 1.88036500 & 3.13089000 & 1.74030100 \\
\hline $\mathrm{C}$ & 2.99249600 & 4.02687800 & 1.86620400 \\
\hline $\mathrm{C}$ & 2.76935900 & 4.85434300 & 3.00928700 \\
\hline $\mathrm{C}$ & 1.51758100 & 4.47788500 & 3.59676400 \\
\hline $\mathrm{C}$ & 0.96951700 & 3.41177900 & 2.81089800 \\
\hline $\mathrm{C}$ & 6.71321400 & 0.07301700 & 3.36006400 \\
\hline $\mathrm{C}$ & 6.05361800 & 0.53019000 & 2.17310400 \\
\hline $\mathrm{C}$ & 4.98464800 & -0.37235300 & 1.87305300 \\
\hline $\mathrm{C}$ & 4.98329200 & -1.39469700 & 2.88545200 \\
\hline $\mathrm{C}$ & 6.05563700 & -1.11766300 & 3.78791300 \\
\hline $\mathrm{H}$ & 5.69518600 & 0.96316400 & 8.25617200 \\
\hline $\mathrm{H}$ & 3.32657300 & 2.12602700 & 8.82718700 \\
\hline $\mathrm{H}$ & 3.23987400 & 4.51413800 & 7.53889300 \\
\hline $\mathrm{H}$ & 5.57251100 & 4.80840500 & 6.19742900 \\
\hline $\mathrm{H}$ & 7.09652900 & 2.62918500 & 6.66341400 \\
\hline $\mathrm{H}$ & 1.69424500 & -1.50886200 & 7.26373100 \\
\hline $\mathrm{H}$ & 1.47658700 & -2.29522500 & 4.68446800 \\
\hline $\mathrm{H}$ & 0.08041400 & -0.38867600 & 3.35367100 \\
\hline $\mathrm{H}$ & -0.61384000 & 1.53079300 & 5.12800300 \\
\hline $\mathrm{H}$ & 0.39727400 & 0.83981800 & 7.53752200 \\
\hline $\mathrm{H}$ & 1.74754900 & 2.38570100 & 0.96651400 \\
\hline $\mathrm{H}$ & 3.85084000 & 4.07265700 & 1.20823600 \\
\hline $\mathrm{H}$ & 3.43042500 & 5.63368900 & 3.36617900 \\
\hline $\mathrm{H}$ & 1.06493300 & 4.92565500 & 4.47212200 \\
\hline $\mathrm{H}$ & 0.02794400 & 2.90976000 & 2.98915600 \\
\hline $\mathrm{H}$ & 7.55838600 & 0.54642000 & 3.84264200 \\
\hline $\mathrm{H}$ & 6.32536200 & 1.40756000 & 1.60015900 \\
\hline $\mathrm{H}$ & 4.31401600 & -0.31174000 & 1.02588300 \\
\hline $\mathrm{H}$ & 4.30326400 & -2.23501700 & 2.94071300 \\
\hline $\mathrm{H}$ & 6.28714400 & -1.67824800 & 4.68517300 \\
\hline $\mathrm{H}$ & 2.74190900 & 0.90349300 & 3.52742500 \\
\hline $\mathrm{H}$ & 4.39372000 & 2.11693800 & 3.12868600 \\
\hline $\mathrm{H}$ & 2.44212700 & 2.29852500 & 5.40466200 \\
\hline $\mathrm{H}$ & 4.14306100 & 3.28825200 & 4.70940300 \\
\hline
\end{tabular}

$\mathbf{T S}_{4 \mathrm{~b}}$

$\mathrm{E}(\mathrm{RB} 3 \mathrm{PW} 91)=-1231.77080772$ A.U.

Thermal correction to Gibbs Free Energy= 0.319430 A.U.

$\begin{array}{lrrr}\mathrm{Ru} & 4.22917200 & 2.19999200 & 6.07641600 \\ \mathrm{Ru} & 2.08692400 & 0.61214200 & 5.25234200 \\ \mathrm{Ru} & 2.89925300 & 2.76367500 & 3.63492700 \\ \mathrm{Ru} & 4.52393700 & 0.47907900 & 3.88964300 \\ \mathrm{O} & 3.97137700 & 0.27673400 & 5.78605900 \\ \mathrm{C} & 5.80301000 & 2.07212500 & 7.54804200 \\ \mathrm{C} & 4.53213200 & 2.18376900 & 8.20831600 \\ \mathrm{C} & 3.96679700 & 3.45821200 & 7.86220600 \\ \mathrm{C} & 4.89319600 & 4.12647000 & 7.00705300 \\ \mathrm{C} & 6.01864300 & 3.27535200 & 6.79768600 \\ \mathrm{C} & 1.15599800 & -0.99362500 & 6.45751400 \\ \mathrm{C} & 0.91014600 & -1.23572900 & 5.07285900 \\ \mathrm{C} & 0.14346800 & -0.12985600 & 4.56097500 \\ \mathrm{C} & -0.06770800 & 0.78546400 & 5.63453800 \\ \mathrm{C} & 0.56377500 & 0.25585200 & 6.81091000 \\ \mathrm{C} & 2.41419400 & 3.08844800 & 1.52891500 \\ \mathrm{C} & 3.36198700 & 4.08486500 & 1.92788800 \\ \mathrm{C} & 2.76981700 & 4.85782200 & 2.97367600 \\ \mathrm{C} & 1.45078100 & 4.35709000 & 3.21719300 \\ \mathrm{C} & 1.23251700 & 3.25629100 & 2.32525800 \\ \mathrm{H} & 4.56990600 & 2.19146600 & 3.63684500 \\ \mathrm{H} & 2.02851300 & 2.33653300 & 5.11126000 \\ \mathrm{H} & 3.73628800 & 3.45248100 & 4.96220100 \\ \mathrm{H} & 6.60894600 & -0.05457500 & 3.38325200 \\ \mathrm{C} & 5.93949500 & 0.42252300 & 2.20617700 \\ \mathrm{H} & 4.84256100 & -0.44605600 & 1.92998200 \\ \mathrm{H} & 4.12935100 & -2.29417400 & 3.00418300 \\ \mathrm{C} & 4.83205100 & -1.47340900 & 2.93890800 \\ \mathrm{C} & 5.92585700 & -1.22801200 & 3.82302500 \\ \mathrm{H} & 6.47867500 & 1.22780400 & 7.60024500 \\ \mathrm{H} & 4.07925100 & 1.44314900 & 8.85525500 \\ \mathrm{H} & 3.01398800 & 3.84835400 & 8.19605900 \\ \mathrm{H} & 4.74863500 & 5.10175200 & 6.55959100 \\ \mathrm{H} & 6.88137500 & 3.50168500 & 6.18433100 \\ \mathrm{H} & 1.74211600 & -1.62319200 & 7.11580100 \\ \mathrm{H} & 1.22812100 & -2.10393800 & 4.50990200 \\ \mathrm{H} & -0.22217200 & -0.02464600 & 3.54788800 \\ \mathrm{H} & -0.60977100 & 1.72071800 & 5.57652400 \\ \mathrm{H} & 0.57560800 & 0.71647000 & 7.79032400 \\ \mathrm{H} & 4.55946200 & 2.34587900 & 0.75506200 \\ \mathrm{H} & 3.23709700 & 5.68820800 & 3.48764400 \\ \mathrm{H} & 0.74504900 & 4.74784400 & 3.93870700 \\ \mathrm{H} & 2.75234600 & 0.38882300 & 3.84369300 \\ \mathrm{H} & & \\ \mathrm{H} & & \end{array}$

$(\mathrm{CpRu})_{4}(\mathrm{H})_{4}(\mathbf{1 c})$

$\mathrm{E}(\mathrm{RB} 3 \mathrm{PW} 91)=-1156.52037207$ A.U.

Thermal correction to Gibbs Free Energy= 0.314276 A.U.

$\begin{array}{llll}\mathrm{Ru} & 4.29834600 \quad 2.32529100 & 5.79329600\end{array}$ 


\begin{tabular}{|c|c|c|c|}
\hline $\mathrm{Ru}$ & 2.37767100 & 0.41721100 & 5.46237000 \\
\hline $\mathrm{Ru}$ & 2.70699300 & 2.36002400 & 3.57872600 \\
\hline $\mathrm{Ru}$ & 4.59120800 & 0.41561200 & 3.86794700 \\
\hline $\mathrm{C}$ & 5.67276100 & 2.35371700 & 7.48898000 \\
\hline $\mathrm{C}$ & 4.42464600 & 2.93546000 & 7.88348400 \\
\hline $\mathrm{C}$ & 4.21464000 & 4.09810500 & 7.06503400 \\
\hline $\mathrm{C}$ & 5.31481800 & 4.22072700 & 6.16890600 \\
\hline $\mathrm{C}$ & 6.22364000 & 3.13420600 & 6.42859800 \\
\hline $\mathrm{C}$ & 1.32008400 & -1.49223300 & 5.47885000 \\
\hline $\mathrm{C}$ & 0.36079900 & -0.41815100 & 5.44135000 \\
\hline $\mathrm{C}$ & 0.53233800 & 0.35613500 & 6.62758000 \\
\hline $\mathrm{C}$ & 1.59633200 & -0.21651600 & 7.39726900 \\
\hline $\mathrm{C}$ & 2.07295500 & -1.36757000 & 6.68155800 \\
\hline $\mathrm{C}$ & 1.35780400 & 2.44966600 & 1.86375100 \\
\hline $\mathrm{C}$ & 2.60762300 & 3.05121700 & 1.51038900 \\
\hline $\mathrm{C}$ & 2.80016300 & 4.18269800 & 2.37642800 \\
\hline $\mathrm{C}$ & 1.68561900 & 4.26621300 & 3.25859900 \\
\hline $\mathrm{C}$ & 0.78661600 & 3.18612700 & 2.94563500 \\
\hline $\mathrm{C}$ & 5.82545400 & -1.38596800 & 3.94788900 \\
\hline $\mathrm{C}$ & 6.66887700 & -0.24002300 & 3.74595400 \\
\hline $\mathrm{C}$ & 6.29106200 & 0.35548100 & 2.49924500 \\
\hline $\mathrm{C}$ & 5.21609900 & -0.39956400 & 1.94126000 \\
\hline $\mathrm{C}$ & 4.93063700 & -1.48427800 & 2.84443900 \\
\hline $\mathrm{H}$ & 6.10909200 & 1.45254400 & 7.90258800 \\
\hline $\mathrm{H}$ & 3.77511900 & 2.58316700 & 8.67387800 \\
\hline $\mathrm{H}$ & 3.35514700 & 4.75573300 & 7.10656400 \\
\hline $\mathrm{H}$ & 5.44541400 & 4.99509300 & 5.42355300 \\
\hline $\mathrm{H}$ & 7.16220100 & 2.95292800 & 5.92135800 \\
\hline $\mathrm{H}$ & 1.44719900 & -2.25885200 & 4.72513800 \\
\hline $\mathrm{H}$ & -0.36829100 & -0.23976700 & 4.66160800 \\
\hline $\mathrm{H}$ & -0.02540400 & 1.24798600 & 6.88655800 \\
\hline $\mathrm{H}$ & 1.95367600 & 0.13431100 & 8.35636100 \\
\hline $\mathrm{H}$ & 2.88289800 & -2.01622300 & 6.99174100 \\
\hline $\mathrm{H}$ & 0.93260300 & 1.56292300 & 1.40927500 \\
\hline $\mathrm{H}$ & 3.27087200 & 2.73292700 & 0.71705600 \\
\hline $\mathrm{H}$ & 3.65598300 & 4.84623600 & 2.37264500 \\
\hline $\mathrm{H}$ & 1.54115600 & 5.00944400 & 4.03262600 \\
\hline $\mathrm{H}$ & -0.15952100 & 2.98103800 & 3.42936900 \\
\hline $\mathrm{H}$ & 5.85053400 & -2.04897600 & 4.80384800 \\
\hline $\mathrm{H}$ & 7.46433900 & 0.09168600 & 4.39995200 \\
\hline $\mathrm{H}$ & 6.72514500 & 1.24969800 & 2.06848000 \\
\hline $\mathrm{H}$ & 4.71619800 & -0.20304300 & 1.00182900 \\
\hline $\mathrm{H}$ & 4.16900900 & -2.24150700 & 2.70788300 \\
\hline $\mathrm{H}$ & 2.74141500 & 0.50525400 & 3.63375500 \\
\hline $\mathrm{H}$ & 4.53087100 & 2.27335700 & 3.94231400 \\
\hline $\mathrm{H}$ & 4.22378100 & 0.46309800 & 5.69050800 \\
\hline $\mathrm{H}$ & 2.47335900 & 2.27873100 & 5.42204400 \\
\hline
\end{tabular}

\section{A1}

$\mathrm{E}(\mathrm{RB} 3 \mathrm{PW} 91)=-1306.87343681 \mathrm{~A} . \mathrm{U}$.

\begin{tabular}{|c|c|c|c|}
\hline \multicolumn{4}{|c|}{ Thermal correction to Gibbs Free Energy $=0.322060$ A.U } \\
\hline $\mathrm{Ru}$ & 4.36534000 & 2.03277400 & 6.12907900 \\
\hline $\mathrm{Ru}$ & 2.14338700 & 0.32741000 & 5.50749900 \\
\hline $\mathrm{Ru}$ & 3.01310100 & 2.36084400 & 3.79577400 \\
\hline $\mathrm{Ru}$ & 4.66984600 & 0.09727600 & 4.21964800 \\
\hline $\mathrm{O}$ & 4.69897500 & 0.03889800 & 6.27064900 \\
\hline $\mathrm{O}$ & 3.51791900 & -0.56523000 & 6.76685200 \\
\hline
\end{tabular}

\begin{tabular}{|c|c|c|c|}
\hline C & 6.19385900 & 2.94340000 & 7.03953700 \\
\hline $\mathrm{C}$ & 5.37354200 & 2.41530200 & 8.08578100 \\
\hline $\mathrm{C}$ & 4.11982500 & 3.08067200 & 8.07835700 \\
\hline $\mathrm{C}$ & 4.14705000 & 4.03730000 & 7.00218700 \\
\hline $\mathrm{C}$ & 5.42531900 & 3.95178200 & 6.37031000 \\
\hline $\mathrm{C}$ & 0.55779600 & -1.19367100 & 5.15163100 \\
\hline $\mathrm{C}$ & 0.30837800 & -0.02143700 & 4.35352600 \\
\hline C & 0.10019500 & 1.07676100 & 5.24248900 \\
\hline $\mathrm{C}$ & 0.22650800 & 0.59614700 & 6.59383100 \\
\hline $\mathrm{C}$ & 0.51484400 & -0.79743700 & 6.51701500 \\
\hline $\mathrm{C}$ & 2.50823600 & 2.82008500 & 1.71600600 \\
\hline $\mathrm{C}$ & 3.54927600 & 3.69875600 & 2.16181600 \\
\hline $\mathrm{C}$ & 3.03710700 & 4.45359800 & 3.26662400 \\
\hline $\mathrm{C}$ & 1.68033200 & 4.04072800 & 3.49696200 \\
\hline $\mathrm{C}$ & 1.35391000 & 3.03369800 & 2.52821600 \\
\hline $\mathrm{C}$ & 6.73462100 & 0.13832900 & 3.22181900 \\
\hline $\mathrm{C}$ & 5.72449800 & -0.10895100 & 2.24443300 \\
\hline $\mathrm{C}$ & 5.04131500 & -1.31795800 & 2.59123900 \\
\hline C & 5.61454900 & -1.81367600 & 3.80941200 \\
\hline $\mathrm{C}$ & 6.65414300 & -0.89664800 & 4.19129000 \\
\hline $\mathrm{H}$ & 7.21141500 & 2.65263700 & 6.81374100 \\
\hline $\mathrm{H}$ & 5.63627800 & 1.58674100 & 8.73267400 \\
\hline $\mathrm{H}$ & 3.29293100 & 2.89252300 & 8.75018000 \\
\hline $\mathrm{H}$ & 3.34744100 & 4.71629400 & 6.73729100 \\
\hline $\mathrm{H}$ & 5.76037400 & 4.54947300 & 5.53232300 \\
\hline $\mathrm{H}$ & 0.74701900 & -2.19366500 & 4.78363700 \\
\hline $\mathrm{H}$ & 0.26454600 & 0.01522300 & 3.27331600 \\
\hline $\mathrm{H}$ & -0.14212500 & 2.09022400 & 4.95451000 \\
\hline $\mathrm{H}$ & 0.10977100 & 1.17652300 & 7.49946800 \\
\hline $\mathrm{H}$ & 0.74180300 & -1.43701200 & 7.36191100 \\
\hline $\mathrm{H}$ & 2.58158700 & 2.11376600 & 0.89860500 \\
\hline $\mathrm{H}$ & 4.54046200 & 3.78363000 & 1.73502900 \\
\hline $\mathrm{H}$ & 3.57443400 & 5.21469200 & 3.81746500 \\
\hline $\mathrm{H}$ & 1.01407800 & 4.43878800 & 4.25230600 \\
\hline $\mathrm{H}$ & 0.40278400 & 2.52676700 & 2.42516100 \\
\hline $\mathrm{H}$ & 7.40829200 & 0.98499300 & 3.24403800 \\
\hline $\mathrm{H}$ & 5.51164100 & 0.51300000 & 1.38465900 \\
\hline $\mathrm{H}$ & 4.24329000 & -1.78130600 & 2.02544000 \\
\hline $\mathrm{H}$ & 5.33996700 & -2.72281300 & 4.32787600 \\
\hline $\mathrm{H}$ & 7.25046300 & -0.96605700 & 5.09268200 \\
\hline $\mathrm{H}$ & 3.50783300 & 0.86122200 & 3.12668000 \\
\hline $\mathrm{H}$ & 4.69979000 & 2.12014800 & 4.38765400 \\
\hline $\mathrm{H}$ & 3.14953500 & -0.62804200 & 4.43094800 \\
\hline & 2.65085100 & 2.02589300 & 5.70466100 \\
\hline
\end{tabular}

$\mathrm{TS}_{\mathrm{A} 1 / \mathrm{A} 2}$

$\mathrm{E}(\mathrm{RB} 3 \mathrm{PW} 91)=-1306.86407426$ A.U.

Thermal correction to Gibbs Free Energy= 0.322850 A.U.

$\begin{array}{lrrr}\mathrm{Ru} & 4.47566400 & 2.03774100 & 6.25065600 \\ \mathrm{Ru} & 2.01405000 & 0.25006100 & 5.32773000 \\ \mathrm{Ru} & 3.10173700 & 2.44459700 & 3.93710000 \\ \mathrm{Ru} & 4.70333300 & 0.08221200 & 4.31632500 \\ \mathrm{O} & 4.75472300 & 0.03546600 & 6.35644900 \\ \mathrm{O} & 3.42317200 & -0.12044500 & 6.86408900 \\ \mathrm{C} & 6.23698900 & 2.85099400 & 7.13027600 \\ \mathrm{C} & 5.33145800 & 2.57895900 & 8.21283900 \\ \mathrm{C} & 4.16312300 & 3.36115200 & 8.01440600\end{array}$




$\begin{array}{rrrr}\mathrm{C} & 4.33428400 & 4.16570100 & 6.84271200 \\ \mathrm{C} & 5.61104300 & 3.83586000 & 6.28112000 \\ \mathrm{C} & 0.69973200 & -1.56676900 & 5.38476200 \\ \mathrm{C} & 0.30760300 & -0.67106000 & 4.33028500 \\ \mathrm{C} & -0.10084200 & 0.56593200 & 4.93163100 \\ \mathrm{C} & 0.04364100 & 0.43734200 & 6.35443400 \\ \mathrm{C} & 0.54490200 & -0.87216400 & 6.61396500 \\ \mathrm{C} & 2.73986000 & 2.92112900 & 1.84784100 \\ \mathrm{C} & 3.80809800 & 3.74723700 & 2.33179400 \\ \mathrm{C} & 3.28742100 & 4.56031400 & 3.38655000 \\ \mathrm{C} & 1.90264400 & 4.23829300 & 3.56309100 \\ \mathrm{C} & 1.56051800 & 3.23731500 & 2.60099200 \\ \mathrm{C} & 6.55208100 & 0.29759600 & 3.10683300 \\ \mathrm{C} & 5.44077500 & 0.12509500 & 2.21413300 \\ \mathrm{C} & 4.84064900 & -1.13084300 & 2.50831700 \\ \mathrm{C} & 5.58064300 & -1.75621400 & 3.57379200 \\ \mathrm{C} & 6.64017600 & -0.85749500 & 3.93696800 \\ \mathrm{H} & 7.22572400 & 2.43032700 & 6.99790600 \\ \mathrm{H} & 5.47492400 & 1.84519000 & 8.99590100 \\ \mathrm{H} & 3.27124400 & 3.32549100 & 8.62839000 \\ \mathrm{H} & 3.61836400 & 4.87369000 & 6.45051400 \\ \mathrm{H} & 6.04986900 & 4.27583300 & 5.39466900 \\ \mathrm{H} & 1.05779800 & -2.58121300 & 5.26802400 \\ \mathrm{H} & 2.39651300 & 1.95573200 & 5.54452600 \\ \mathrm{H} & 0.28127700 & -0.90435900 & 3.27354000 \\ \mathrm{H} & -0.48066700 & 1.43380700 & 4.40906000 \\ \mathrm{H} & -0.18465500 & 1.19213000 & 7.09524100 \\ \mathrm{H} & 0.83757000 & -1.24998600 & 7.58634200 \\ \mathrm{H} & 2.80059000 & 2.20738400 & 1.03610700 \\ \mathrm{H} & 4.82288100 & 3.76592200 & 1.95486900 \\ \mathrm{H} & 3.84587900 & 5.29178200 & 3.95687800 \\ \mathrm{H} & 1.23066500 & 4.68898300 & 4.28252700 \\ \mathrm{H} & 0.58338200 & 2.79363200 & 2.46218600 \\ \mathrm{H} & 7.20664600 & 1.15922400 & 3.14656400 \\ \mathrm{H} & 5.10896800 & 0.82985100 & 1.46491700 \\ \mathrm{H} & 5.36934700 & -1.54491200 & 2.01643200 \\ \mathrm{H} & 7.36862700 & -1.02034400 & 4.72186500 \\ \mathrm{H} & 2.32248500 & 0.91733200 & 3.74928100 \\ \mathrm{H} & & & \\ \mathrm{H} & 3.16891300 & -0.73283900 & 4.54008000 \\ \mathrm{H} & & \\ \mathrm{H} & & \end{array}$

\section{A2}

$\mathrm{E}(\mathrm{RB} 3 \mathrm{PW} 91)=-1306.87984812 \mathrm{~A} . \mathrm{U}$.

\begin{tabular}{|c|c|c|c|}
\hline \multicolumn{4}{|c|}{ Thermal correction to Gibbs Free Energy= 0.323201 A.L } \\
\hline $\mathrm{Ru}$ & 4.03842700 & 2.10687500 & 6.63268900 \\
\hline $\mathrm{Ru}$ & 2.32065200 & 0.23778700 & 5.38725300 \\
\hline $\mathrm{Ru}$ & 3.46234900 & 2.52234900 & 3.94077400 \\
\hline $\mathrm{Ru}$ & 4.72900800 & -0.00298900 & 3.87696200 \\
\hline $\mathrm{O}$ & 5.07333600 & 0.41433600 & 5.90617300 \\
\hline $\mathrm{O}$ & 3.89739200 & 0.08628400 & 6.70743800 \\
\hline $\mathrm{C}$ & 5.54951700 & 3.47533600 & 7.45993700 \\
\hline $\mathrm{C}$ & 4.91595000 & 2.75902300 & 8.52116900 \\
\hline $\mathrm{C}$ & 3.51362600 & 3.08174100 & 8.49484700 \\
\hline $\mathrm{C}$ & 3.30043800 & 3.99335000 & 7.41268600 \\
\hline $\mathrm{C}$ & 4.56552500 & 4.25405300 & 6.78368500 \\
\hline $\mathrm{C}$ & 1.01629300 & -1.47871900 & 5.63575400 \\
\hline
\end{tabular}

\begin{tabular}{rrrr}
$\mathrm{C}$ & 0.69284300 & -0.80739600 & 4.40256400 \\
$\mathrm{C}$ & 0.23260700 & 0.50595000 & 4.71434500 \\
$\mathrm{C}$ & 0.27459700 & 0.66024100 & 6.14022200 \\
$\mathrm{C}$ & 0.75756900 & -0.55672400 & 6.70313600 \\
$\mathrm{C}$ & 2.35495300 & 3.02872500 & 2.12404900 \\
$\mathrm{C}$ & 3.72280500 & 3.40043500 & 1.95690700 \\
$\mathrm{C}$ & 4.04595300 & 4.36587500 & 2.96827500 \\
$\mathrm{C}$ & 2.85286600 & 4.62145700 & 3.72989200 \\
$\mathrm{C}$ & 1.81459400 & 3.79552600 & 3.21366900 \\
$\mathrm{C}$ & 6.54192800 & -0.15295300 & 2.61201200 \\
$\mathrm{C}$ & 5.35364900 & -0.30980700 & 1.81181500 \\
$\mathrm{C}$ & 4.66859900 & -1.47017200 & 2.28029700 \\
$\mathrm{C}$ & 5.43187200 & -2.03995300 & 3.36395500 \\
$\mathrm{C}$ & 6.57773000 & -1.21749400 & 3.55716400 \\
$\mathrm{H}$ & 6.59725900 & 3.41258900 & 7.19225000 \\
$\mathrm{H}$ & 5.40541300 & 2.07977000 & 9.20809900 \\
$\mathrm{H}$ & 2.75768400 & 2.71894500 & 9.17994200 \\
$\mathrm{H}$ & 2.35030800 & 4.42762800 & 7.12863500 \\
$\mathrm{H}$ & 4.74636100 & 4.91636400 & 5.95019100 \\
$\mathrm{H}$ & 1.35802900 & -2.50035000 & 5.73964400 \\
$\mathrm{H}$ & 0.77756000 & -1.22934800 & 3.40910600 \\
$\mathrm{H}$ & -0.08473800 & 1.25393300 & 4.00083900 \\
$\mathrm{H}$ & 0.00331200 & 1.55127600 & 6.69175500 \\
$\mathrm{H}$ & 0.92237800 & -0.74514400 & 7.75696800 \\
$\mathrm{H}$ & 1.81297200 & 2.31115800 & 1.52093400 \\
$\mathrm{H}$ & 4.40361200 & 3.00866800 & 1.21271700 \\
$\mathrm{H}$ & 5.00510500 & 4.85154300 & 3.09831300 \\
$\mathrm{H}$ & 2.75681200 & 5.32307300 & 4.54868200 \\
$\mathrm{H}$ & 0.79527000 & 3.75511400 & 3.57637400 \\
$\mathrm{H}$ & 7.27993800 & 0.63273500 & 2.51430100 \\
$\mathrm{H}$ & 5.04982500 & 0.32135800 & 0.98726100 \\
$\mathrm{H}$ & 3.74798800 & -1.86932600 & 1.87365800 \\
& 5.19079500 & -2.93711900 & 3.91904600 \\
$\mathrm{H}$ & 7.32068500 & -1.34441800 & 4.33511200 \\
$\mathrm{H}$ & 5.00158400 & 1.72056100 & 3.80253000 \\
$\mathrm{H}$ & 2.63755500 & 2.08490900 & 5.46723500 \\
\hline
\end{tabular}

$\mathbf{T S}_{\mathrm{A} 2 / \mathrm{A3}}$

$\mathrm{E}(\mathrm{RB} 3 \mathrm{PW} 91)=-1306.86753185$ A.U.

Thermal correction to Gibbs Free Energy= 0.320772 A.U.

$\begin{array}{llll}\mathrm{Ru} & 4.00371600 & 2.05592200 & 6.55315100 \\ \mathrm{Ru} & 2.32993600 & 0.25407300 & 5.34137400 \\ \mathrm{Ru} & 3.48582700 & 2.57938400 & 3.94851100 \\ \mathrm{Ru} & 4.73451600 & 0.03912100 & 3.88974100 \\ \mathrm{O} & 5.27239100 & 0.70134600 & 5.58417300 \\ \mathrm{O} & 3.54750300 & 0.19688600 & 6.88689900 \\ \mathrm{C} & 5.56987800 & 3.32545600 & 7.47951000 \\ \mathrm{C} & 4.88659500 & 2.58280700 & 8.49084400 \\ \mathrm{C} & 3.50618300 & 2.98336000 & 8.47601100 \\ \mathrm{C} & 3.35728200 & 3.97143600 & 7.45318100 \\ \mathrm{C} & 4.64035400 & 4.20454000 & 6.85592100 \\ \mathrm{C} & 0.98603700 & -1.46131500 & 5.67747100 \\ \mathrm{C} & 0.71687600 & -0.88370900 & 4.38853600 \\ \mathrm{C} & 0.22475800 & 0.44247700 & 4.59299500 \\ \mathrm{C} & 0.18208900 & 0.68689100 & 6.00341100\end{array}$




$\begin{array}{lrrr}\text { C } & 0.65291800 & -0.48073600 & 6.66113300 \\ \mathrm{C} & 2.40713900 & 3.17109500 & 2.10802600 \\ \mathrm{C} & 3.77144600 & 3.55354600 & 1.99048300 \\ \mathrm{C} & 4.08147100 & 4.46434200 & 3.05759200 \\ \mathrm{C} & 2.88516700 & 4.67077500 & 3.82311500 \\ \mathrm{C} & 1.86207200 & 3.85028700 & 3.25092900 \\ \mathrm{C} & 6.38668200 & -0.11808900 & 2.42765000 \\ \mathrm{C} & 5.17539800 & -0.56250700 & 1.77942300 \\ \mathrm{C} & 4.72573100 & -1.71743600 & 2.47114600 \\ \mathrm{C} & 5.65162200 & -2.00423900 & 3.53125800 \\ \mathrm{C} & 6.67930600 & -1.02528200 & 3.48859700 \\ \mathrm{H} & 6.61255000 & 3.21310900 & 7.20989800 \\ \mathrm{H} & 5.32758100 & 1.82669100 & 9.12780100 \\ \mathrm{H} & 2.72516400 & 2.61660800 & 9.12964200 \\ \mathrm{H} & 2.43618400 & 4.48002700 & 7.19748600 \\ \mathrm{H} & 4.86692400 & 4.90816400 & 6.06755400 \\ \mathrm{H} & 1.34598900 & -2.46309500 & 5.87130700 \\ \mathrm{H} & 0.84158900 & -1.37693800 & 3.43293800 \\ \mathrm{H} & -0.07399100 & 1.13525900 & 3.81743900 \\ \mathrm{H} & -0.13129700 & 1.60420700 & 6.48502000 \\ \mathrm{H} & 0.80409700 & -0.58611300 & 7.72782500 \\ \mathrm{H} & 1.87729800 & 2.48495500 & 1.45982200 \\ \mathrm{H} & 4.46667900 & 3.20304700 & 1.23842400 \\ \mathrm{H} & 5.04003100 & 4.94078300 & 3.22153300 \\ \mathrm{H} & 2.77338400 & 5.33239300 & 4.67185200 \\ \mathrm{H} & 0.84088100 & 3.77324000 & 3.60331800 \\ \mathrm{H} & 6.99483600 & 0.72652300 & 2.12976900 \\ \mathrm{H} & 4.71158700 & -0.10953000 & 0.91301200 \\ \mathrm{H} & 3.83316000 & -2.28723500 & 2.24427700 \\ \mathrm{H} & 5.57826100 & -2.81835700 & 4.24125100 \\ \mathrm{H} & 7.49860300 & -0.93670000 & 4.19045000 \\ \mathrm{H} & 3.05956500 & 0.83650700 & 3.69476200 \\ \mathrm{H} & 4.86715500 & 1.67674500 & 3.42033700 \\ \mathrm{H} & 3.50174700 & -0.82743400 & 4.86516200 \\ \mathrm{H} & 2.59746300 & 2.12910400 & 5.47227700 \\ & & & \end{array}$

\section{A3}

$\mathrm{E}(\mathrm{RB} 3 \mathrm{PW} 91)=-1306.96361984$ A.U.

\begin{tabular}{|c|c|c|c|}
\hline \multicolumn{4}{|c|}{ Thermal correction to Gibbs Free Energy $=0.323350$ A.U } \\
\hline $\mathrm{Ru}$ & 3.93360500 & 2.27269000 & 6.31433000 \\
\hline $\mathrm{Ru}$ & 2.27768300 & 0.31868100 & 5.18533300 \\
\hline $\mathrm{Ru}$ & 3.38268000 & 2.51454700 & 3.58125900 \\
\hline $\mathrm{Ru}$ & 4.81230200 & 0.15583200 & 4.02619600 \\
\hline $\mathrm{O}$ & 5.05347400 & 2.10956700 & 4.60990400 \\
\hline $\mathrm{O}$ & 4.12636000 & 0.26998600 & 5.95711100 \\
\hline $\mathrm{C}$ & 5.17291300 & 2.81941300 & 8.01835500 \\
\hline $\mathrm{C}$ & 3.91980800 & 2.30295800 & 8.49661400 \\
\hline $\mathrm{C}$ & 2.87905100 & 3.16374200 & 8.04184600 \\
\hline $\mathrm{C}$ & 3.47906700 & 4.21837200 & 7.27081500 \\
\hline $\mathrm{C}$ & 4.88694200 & 3.99997900 & 7.25291500 \\
\hline $\mathrm{C}$ & 0.81694000 & -1.26004500 & 4.69092500 \\
\hline $\mathrm{C}$ & 0.09924000 & -0.02607500 & 4.71849400 \\
\hline $\mathrm{C}$ & 0.25184800 & 0.53538500 & 6.02002100 \\
\hline $\mathrm{C}$ & 1.03090300 & -0.37385900 & 6.81571900 \\
\hline $\mathrm{C}$ & 1.37752900 & -1.49326500 & 5.99223000 \\
\hline $\mathrm{C}$ & 1.82844900 & 3.39279200 & 2.20487400 \\
\hline $\mathrm{C}$ & 3.03083900 & 3.11445400 & 1.48705000 \\
\hline
\end{tabular}

$\begin{array}{lrrr}\text { C } & 4.09382100 & 3.89570000 & 2.05338500 \\ \mathrm{C} & 3.54643300 & 4.63382100 & 3.15200700 \\ \mathrm{C} & 2.14930000 & 4.30939300 & 3.24877900 \\ \mathrm{C} & 6.75026700 & -0.02148600 & 3.00979900 \\ \mathrm{C} & 5.80600900 & -0.73527200 & 2.21293600 \\ \mathrm{C} & 5.27007100 & -1.79751800 & 3.00416200 \\ \mathrm{C} & 5.88164900 & -1.74291800 & 4.29209300 \\ \mathrm{C} & 6.80933400 & -0.64729700 & 4.30091600 \\ \mathrm{H} & 6.15268600 & 2.39994300 & 8.20768000 \\ \mathrm{H} & 3.79119100 & 1.40667300 & 9.09097900 \\ \mathrm{H} & 1.82198100 & 3.04708700 & 8.24482600 \\ \mathrm{H} & 2.95435700 & 5.03573200 & 6.79278700 \\ \mathrm{H} & 5.61822500 & 4.60965100 & 6.73618700 \\ \mathrm{H} & 0.90219400 & -1.91950000 & 3.83629700 \\ \mathrm{H} & -0.43629400 & 0.41769100 & 3.88940800 \\ \mathrm{H} & -0.15838000 & 1.47981600 & 6.35421600 \\ \mathrm{H} & 1.30479500 & -0.23547900 & 7.85330200 \\ \mathrm{H} & 1.95472500 & -2.35756000 & 6.29404100 \\ \mathrm{H} & 0.85567200 & 2.96066400 & 2.00992000 \\ \mathrm{H} & 3.11804900 & 2.44316200 & 0.64190100 \\ \mathrm{H} & 5.12035100 & 3.92156500 & 1.71147600 \\ \mathrm{H} & 4.08555100 & 5.32011100 & 3.79208800 \\ \mathrm{H} & 1.45593200 & 4.70155600 & 3.98182400 \\ \mathrm{H} & 7.31029000 & 0.85188400 & 2.70103200 \\ \mathrm{H} & 5.53483800 & -0.50739700 & 1.19010700 \\ \mathrm{H} & 4.52261000 & -2.51218000 & 2.68439700 \\ \mathrm{H} & 5.66599300 & -2.39862800 & 5.12592700 \\ \mathrm{H} & 7.43395800 & -0.34422300 & 5.13075100 \\ \mathrm{H} & 2.10239300 & 1.33776100 & 3.77827000 \\ \mathrm{H} & 4.01495400 & 1.06049000 & 2.78869700 \\ \mathrm{H} & 3.22436900 & -0.51225400 & 3.93688800 \\ \mathrm{H} & 2.52137700 & 2.25625000 & 5.30316600 \\ & & & \end{array}$

$\mathrm{TS}_{\mathrm{A} 3 / \mathrm{A} 4}$

$\mathrm{E}(\mathrm{RB} 3 \mathrm{PW} 91)=-1306.92708925$ A.U.

Thermal correction to Gibbs Free Energy= 0.321272 A.U

$\begin{array}{llll}\mathrm{Ru} & 3.94776900 & 2.13425000 & 5.97470600 \\ \mathrm{Ru} & 2.49128100 & 0.03297900 & 5.62808800 \\ \mathrm{Ru} & 2.85930500 & 2.54321000 & 3.23297600 \\ \mathrm{Ru} & 4.64568500 & 0.30791900 & 3.88051100 \\ \mathrm{O} & 4.48219000 & 0.20294500 & 5.84470100 \\ \mathrm{O} & 2.13319300 & 1.95210300 & 5.06095700 \\ \mathrm{C} & 4.44775700 & 2.44758300 & 8.05373600 \\ \mathrm{C} & 3.39071400 & 3.34662700 & 7.68315700 \\ \mathrm{C} & 3.91086200 & 4.25049000 & 6.69948900 \\ \mathrm{C} & 5.27132400 & 3.90139400 & 6.46002700 \\ \mathrm{C} & 5.61443400 & 2.80404400 & 7.29707400 \\ \mathrm{C} & 1.83101600 & -2.03739100 & 5.83028400 \\ \mathrm{C} & 0.73213000 & -1.23485000 & 5.34332400 \\ \mathrm{C} & 0.40543000 & -0.29478800 & 6.36542000 \\ \mathrm{C} & 1.26945100 & -0.50568300 & 7.47927000 \\ \mathrm{C} & 2.15153600 & -1.57508100 & 7.14233000 \\ \mathrm{C} & 1.57667700 & 3.79997800 & 2.01713800 \\ \mathrm{C} & 2.69883700 & 3.35487400 & 1.22433200 \\ \mathrm{C} & 3.89527100 & 3.82382500 & 1.83026400 \\ \mathrm{C} & 3.52516600 & 4.53217100 & 3.03545700 \\ \mathrm{C} & 2.08770800 & 4.53754100 & 3.12666400\end{array}$




$\begin{array}{rrrr}\mathrm{C} & 6.67519500 & -0.37084800 & 3.42881900 \\ \mathrm{C} & 6.32094300 & 0.67580600 & 2.52115600 \\ \mathrm{C} & 5.21400400 & 0.23087400 & 1.72076700 \\ \mathrm{C} & 4.85873800 & -1.06904300 & 2.17877100 \\ \mathrm{C} & 5.76202000 & -1.45706900 & 3.22451800 \\ \mathrm{H} & 4.38411100 & 1.65300700 & 8.78629200 \\ \mathrm{H} & 2.38506200 & 3.35256800 & 8.08399500 \\ \mathrm{H} & 3.36051200 & 5.04253000 & 6.20898900 \\ \mathrm{H} & 5.92857400 & 4.36819900 & 5.73656600 \\ \mathrm{H} & 6.57212400 & 2.30041500 & 7.32425900 \\ \mathrm{H} & 2.29915200 & -2.86288900 & 5.30982100 \\ \mathrm{H} & 0.22619900 & -1.35012100 & 4.39369300 \\ \mathrm{H} & -0.33764500 & 0.48918400 & 6.28642400 \\ \mathrm{H} & 1.26781200 & 0.05726300 & 8.40385400 \\ \mathrm{H} & 2.95707500 & -1.94884100 & 7.76264200 \\ \mathrm{H} & 0.53152200 & 3.61336500 & 1.80177100 \\ \mathrm{H} & 2.63523000 & 2.75637800 & 0.32304300 \\ \mathrm{H} & 4.90239200 & 3.66239900 & 1.46821300 \\ \mathrm{H} & 4.20910400 & 5.03986500 & 3.70348100 \\ \mathrm{H} & 1.49722000 & 4.98930100 & 3.91439800 \\ \mathrm{H} & 7.48149200 & -0.34198500 & 4.15112800 \\ \mathrm{H} & 6.82032000 & 1.63263200 & 2.43409200 \\ \mathrm{H} & 4.73019000 & 0.78532500 & 0.92946200 \\ \mathrm{H} & 4.04143200 & -1.66989100 & 1.79859500 \\ \mathrm{H} & 5.76093300 & -2.40346000 & 3.75031200 \\ \mathrm{H} & 2.96155600 & -0.17760600 & 3.97800300 \\ \mathrm{H} & 4.43819900 & 2.09454500 & 4.13324500 \\ \mathrm{H} & 1.98642000 & 0.69273900 & 2.40742500 \\ \mathrm{H} & 1.38202200 & 1.13375800 & 2.58875900\end{array}$

\section{$\mathbf{A 4}+\mathrm{H}_{\mathbf{2}}$}

$\mathrm{E}(\mathrm{RB} 3 \mathrm{PW} 91)=-1306.94377729$ A.U.

Thermal correction to Gibbs Free Energy= 0.308965 A.U.

$\begin{array}{lrrr}\mathrm{Ru} & 4.28514000 & 2.24197900 & 6.25615900 \\ \mathrm{Ru} & 2.21537300 & 0.71392900 & 5.74735700 \\ \mathrm{Ru} & 2.75244700 & 2.89038100 & 3.87094700 \\ \mathrm{Ru} & 4.43714800 & 0.54984600 & 3.87254100 \\ \mathrm{O} & 4.18258100 & 0.27523100 & 5.80626300 \\ \mathrm{O} & 2.38211400 & 2.72651000 & 5.79747700 \\ \mathrm{C} & 4.79746800 & 2.50360000 & 8.41152400 \\ \mathrm{C} & 4.48198500 & 3.77074400 & 7.82867000 \\ \mathrm{C} & 5.41140700 & 4.03553800 & 6.77767900 \\ \mathrm{C} & 6.32007600 & 2.91407100 & 6.71040800 \\ \mathrm{C} & 5.93633400 & 1.98604000 & 7.71965000 \\ \mathrm{C} & 1.03721300 & -1.10112100 & 5.46820800 \\ \mathrm{C} & 0.16009900 & 0.04506900 & 5.39773000 \\ \mathrm{C} & 0.19447100 & 0.69298900 & 6.66560500 \\ \mathrm{C} & 1.08384100 & -0.02397900 & 7.52462200 \\ \mathrm{C} & 1.59646400 & -1.13234400 & 6.78091500 \\ \mathrm{C} & 1.10962900 & 3.70668100 & 2.72357900 \\ \mathrm{C} & 2.02611700 & 3.06355000 & 1.81488900 \\ \mathrm{C} & 3.27979600 & 3.72212300 & 1.89679200 \\ \mathrm{C} & 3.16300100 & 4.76305100 & 2.88578600 \\ \mathrm{C} & 1.81443800 & 4.77120100 & 3.37034500 \\ \mathrm{C} & 6.34747700 & -0.24662500 & 3.23721000 \\ \mathrm{C} & 5.92785700 & 0.76549200 & 2.30203700 \\ \mathrm{C} & 4.71463800 & 0.34740000 & 1.68851000\end{array}$

$\begin{array}{lrrr}\mathrm{C} & 4.34212200 & -0.90511400 & 2.28701500 \\ \mathrm{C} & 5.36565100 & -1.28765700 & 3.21668200 \\ \mathrm{H} & 4.27343100 & 2.02495700 & 9.22867400 \\ \mathrm{H} & 3.64265100 & 4.39803700 & 8.10256800 \\ \mathrm{H} & 5.44565800 & 4.92185600 & 6.15795100 \\ \mathrm{H} & 7.14836500 & 2.80530700 & 6.02266300 \\ \mathrm{H} & 6.38571900 & 1.01493700 & 7.88711800 \\ \mathrm{H} & 1.21654700 & -1.81615000 & 4.67618700 \\ \mathrm{H} & -0.42605700 & 0.34679400 & 4.53955600 \\ \mathrm{H} & -0.31302700 & 1.61641300 & 6.91619000 \\ \mathrm{H} & 1.32052100 & 0.22407700 & 8.55116800 \\ \mathrm{H} & 2.33150000 & -1.84334300 & 7.13763100 \\ \mathrm{H} & 0.06877100 & 3.44577000 & 2.86841800 \\ \mathrm{H} & 1.79433400 & 2.21059000 & 1.18956100 \\ \mathrm{H} & 4.17532700 & 3.47066400 & 1.34498500 \\ \mathrm{H} & 3.94965200 & 5.44607900 & 3.18142300 \\ \mathrm{H} & 1.41027800 & 5.44150900 & 4.11916800 \\ \mathrm{H} & 7.24961700 & -0.22689000 & 3.83576300 \\ \mathrm{H} & 6.45281000 & 1.69161300 & 2.10241800 \\ \mathrm{H} & 4.15471800 & 0.88639800 & 0.93705000 \\ \mathrm{H} & 3.45541700 & -1.48000300 & 2.04943000 \\ \mathrm{H} & 5.37812300 & -2.18955800 & 3.81630300 \\ \mathrm{H} & 2.61810200 & 1.01622600 & 3.90521100 \\ \mathrm{H} & 4.48476500 & 2.36999000 & 4.36207100 \\ & 3.76221700 & -4.83921400 & -1.07426400 \\ \mathrm{H} & 4.17532100 & -4.30209900 & -0.76344400\end{array}$

A4

$\mathrm{E}(\mathrm{RB} 3 \mathrm{PW} 91)=-1305.76475186$ A.U.

Thermal correction to Gibbs Free Energy= 0.306329 A.U.

Thermal correction to Enthalpy= 0.389367 A.U.

Entropy $($ Total $)=174.768 \mathrm{Cal} / \mathrm{Mol}-$ Kelvin

$\begin{array}{lrrr}\mathrm{Ru} & 4.24683500 & 2.27073100 & 6.06841100 \\ \mathrm{Ru} & 2.35769700 & 0.47910800 & 5.77062300 \\ \mathrm{Ru} & 2.66296400 & 2.43506800 & 3.62381800 \\ \mathrm{Ru} & 4.60897700 & 0.32380300 & 3.92789000 \\ \mathrm{O} & 4.36269900 & 0.26493300 & 5.88085400 \\ \mathrm{O} & 2.30452100 & 2.48592200 & 5.55975500 \\ \mathrm{C} & 5.76956700 & 2.37388400 & 7.65574900 \\ \mathrm{C} & 4.58303900 & 3.00051000 & 8.15005300 \\ \mathrm{C} & 4.30389400 & 4.11699000 & 7.30220000 \\ \mathrm{C} & 5.29881700 & 4.18017800 & 6.28561900 \\ \mathrm{C} & 6.22075500 & 3.08961800 & 6.50580600 \\ \mathrm{C} & 1.31530600 & -1.44145400 & 5.66820400 \\ \mathrm{C} & 0.35786400 & -0.36475500 & 5.55434000 \\ \mathrm{C} & 0.39591900 & 0.37319100 & 6.77485400 \\ \mathrm{C} & 1.36266600 & -0.22381800 & 7.64258600 \\ \mathrm{C} & 1.92050400 & -1.34556300 & 6.95441300 \\ \mathrm{C} & 1.89941500 & 2.24672300 & 1.58527800 \\ \mathrm{C} & 3.08701700 & 3.02156800 & 1.53804200 \\ \mathrm{C} & 2.88730900 & 4.17679900 & 2.37467700 \\ \mathrm{C} & 1.55016100 & 4.13325600 & 2.88873600 \\ \mathrm{C} & 0.93855200 & 2.92818700 & 2.41670900 \\ \mathrm{C} & 5.80842900 & -1.42890000 & 3.52358700 \\ \mathrm{C} & 6.61607600 & -0.25755600 & 3.36813000 \\ \mathrm{C} & 6.04498600 & 0.52736900 & 2.30321600 \\ \mathrm{C} & 4.90920800 & -0.15673600 & 1.79140000\end{array}$




$\begin{array}{rrrr}\mathrm{C} & 4.73639600 & -1.35037600 & 2.57475400 \\ \mathrm{H} & 6.21778000 & 1.47218800 & 8.05461900 \\ \mathrm{H} & 4.00489700 & 2.68938300 & 9.01056200 \\ \mathrm{H} & 3.43750000 & 4.76241500 & 7.37862400 \\ \mathrm{H} & 5.36177600 & 4.91831600 & 5.49685000 \\ \mathrm{H} & 7.10210400 & 2.87150800 & 5.91733600 \\ \mathrm{H} & 1.52232300 & -2.19223100 & 4.91698200 \\ \mathrm{H} & -0.28134800 & -0.16730200 & 4.70401900 \\ \mathrm{H} & -0.16827200 & 1.27313100 & 6.98661500 \\ \mathrm{H} & 1.62152100 & 0.10959400 & 8.63918000 \\ \mathrm{H} & 2.71979800 & -1.97568000 & 7.32470800 \\ \mathrm{H} & 1.74049500 & 1.29537100 & 1.09302800 \\ \mathrm{H} & 3.99371800 & 2.77640100 & 1.00201100 \\ \mathrm{H} & 3.61064400 & 4.96393100 & 2.54877300 \\ \mathrm{H} & 1.09455300 & 4.86560700 & 3.54404900 \\ \mathrm{H} & -0.07148400 & 2.59779000 & 2.62451300 \\ \mathrm{H} & 5.96318500 & -2.21953800 & 4.24753500 \\ \mathrm{H} & 7.50510600 & -0.01193800 & 3.93524900 \\ \mathrm{H} & 6.42011200 & 1.48277200 & 1.95765700 \\ \mathrm{H} & 4.26665600 & 0.17584700 & 0.98817400 \\ \mathrm{H} & 3.95184100 & -2.08505700 & 2.44131500 \\ \mathrm{H} & 2.74404400 & 0.57570500 & 3.90808900 \\ \mathrm{H} & 4.44346200 & 2.17448000 & 4.16887100\end{array}$

\section{$\mathbf{T S}_{\mathrm{A} 4 / 5}$}

$\mathrm{E}(\mathrm{RB} 3 \mathrm{PW} 91)=-1305.75522578$ A.U.

Thermal correction to Gibbs Free Energy= 0.307512 A.U.

$\begin{array}{lrrr}\mathrm{Ru} & 4.35408600 & 2.26335400 & 6.06054000 \\ \mathrm{Ru} & 2.19924700 & 0.44105500 & 5.62748700 \\ \mathrm{Ru} & 2.73486800 & 2.46840200 & 3.71754400 \\ \mathrm{Ru} & 4.54667700 & 0.32570200 & 4.00650000 \\ \mathrm{O} & 4.23654500 & 0.27259200 & 5.95384300 \\ \mathrm{O} & 2.33053300 & 2.43200100 & 5.64375000 \\ \mathrm{C} & 5.95322200 & 2.52632400 & 7.49950400 \\ \mathrm{C} & 4.74363600 & 2.97619400 & 8.11982800 \\ \mathrm{C} & 4.25108500 & 4.07780500 & 7.34892900 \\ \mathrm{C} & 5.14215900 & 4.33733800 & 6.27627900 \\ \mathrm{C} & 6.19046200 & 3.35148000 & 6.34690600 \\ \mathrm{C} & 1.36880600 & -1.62287400 & 5.44286700 \\ \mathrm{C} & 0.36373400 & -0.61351000 & 5.22498100 \\ \mathrm{C} & 0.21564000 & 0.14027300 & 6.43980500 \\ \mathrm{C} & 1.15193700 & -0.37589000 & 7.39286400 \\ \mathrm{C} & 1.85942800 & -1.44974000 & 6.76030800 \\ \mathrm{C} & 1.56638200 & 2.48217700 & 1.86784100 \\ \mathrm{C} & 2.89013600 & 2.89303000 & 1.53330200 \\ \mathrm{C} & 3.18598300 & 4.06115600 & 2.30420200 \\ \mathrm{C} & 2.02023100 & 4.41194800 & 3.06725000 \\ \mathrm{C} & 1.01558900 & 3.42632900 & 2.79827700 \\ \mathrm{C} & 5.99607100 & -1.30700600 & 3.79389300 \\ \mathrm{C} & 6.59025100 & -0.06635100 & 3.39987800 \\ \mathrm{C} & 5.85490200 & 0.43029500 & 2.26268100 \\ \mathrm{C} & 4.81079800 & -0.49309400 & 1.97696400 \\ \mathrm{C} & 4.88978800 & -1.56511200 & 2.93174400 \\ \mathrm{H} & 6.57219900 & 1.70282100 & 7.83379800 \\ \mathrm{H} & 4.28179700 & 2.56209300 & 9.00677500 \\ \mathrm{H} & 3.30862200 & 4.58424700 & 7.51795100 \\ \mathrm{H} & 5.03714700 & 5.10995800 & 5.52663500\end{array}$

$\begin{array}{rrrr}\mathrm{H} & 7.03503700 & 3.27773800 & 5.67332000 \\ \mathrm{H} & 1.71076300 & -2.35211000 & 4.72101200 \\ \mathrm{H} & -0.21130200 & -0.47998900 & 4.31725900 \\ \mathrm{H} & -0.47398500 & 0.95912000 & 6.60408900 \\ \mathrm{H} & 1.29895700 & -0.02641100 & 8.40664400 \\ \mathrm{H} & 2.69006400 & -1.99056500 & 7.19714000 \\ \mathrm{H} & 1.05753600 & 1.60847900 & 1.47980600 \\ \mathrm{H} & 3.56520300 & 2.38445100 & 0.85857700 \\ \mathrm{H} & 4.12206600 & 4.60588500 & 2.29129900 \\ \mathrm{H} & 1.91850500 & 5.26696400 & 3.72359900 \\ \mathrm{H} & 0.01939500 & 3.39399600 & 3.22140300 \\ \mathrm{H} & 6.30275500 & -1.91881600 & 4.63344700 \\ \mathrm{H} & 7.45770000 & 0.39929800 & 3.84996600 \\ \mathrm{H} & 6.06419500 & 1.34573200 & 1.72461000 \\ \mathrm{H} & 4.07371300 & -0.40152400 & 1.18968100 \\ \mathrm{H} & 4.23324400 & -2.42490300 & 2.97642800 \\ \mathrm{H} & 2.70530300 & 0.63136000 & 3.82941000 \\ \mathrm{H} & 4.51888000 & 2.14966400 & 4.19767100\end{array}$

\section{5}

$\mathrm{E}(\mathrm{RB} 3 \mathrm{PW} 91)=-1305.7774666$ A.U.

Thermal correction to Gibbs Free Energy= 0.309644 A.U. Thermal correction to Enthalpy= 0.390983 A.U.

Entropy $($ Total $)=171.194 \mathrm{Cal} /$ Mol-Kelvin

$\begin{array}{lrrr}\mathrm{Ru} & 4.50622300 & 2.42452500 & 5.86103500 \\ \mathrm{Ru} & 2.18910300 & 0.33432800 & 5.52176100 \\ \mathrm{Ru} & 2.69151400 & 2.39817100 & 3.83459000 \\ \mathrm{Ru} & 4.51076500 & 0.36141000 & 4.09993400 \\ \mathrm{O} & 4.15151500 & 0.43847400 & 6.03710500 \\ \mathrm{O} & 2.48469100 & 2.31326500 & 5.79952700 \\ \mathrm{C} & 6.29917800 & 2.57647800 & 7.09682500 \\ \mathrm{C} & 5.17191800 & 3.11507000 & 7.81232700 \\ \mathrm{C} & 4.71043200 & 4.25825700 & 7.07798400 \\ \mathrm{C} & 5.54924000 & 4.43511300 & 5.94259400 \\ \mathrm{C} & 6.52807200 & 3.39381000 & 5.95038700 \\ \mathrm{C} & 1.24494800 & -1.72458100 & 5.40495000 \\ \mathrm{C} & 0.31064300 & -0.72721300 & 4.98249700 \\ \mathrm{C} & 0.09069900 & 0.17117400 & 6.07233800 \\ \mathrm{C} & 0.89970300 & -0.27234400 & 7.17869200 \\ \mathrm{C} & 1.61322000 & -1.43475100 & 6.74742000 \\ \mathrm{C} & 1.80019200 & 2.53178400 & 1.83998100 \\ \mathrm{C} & 2.88742300 & 3.46008600 & 1.91724800 \\ \mathrm{C} & 2.56308100 & 4.43605600 & 2.91777500 \\ \mathrm{C} & 1.28302500 & 4.11113400 & 3.44776700 \\ \mathrm{C} & 0.80549700 & 2.93840300 & 2.79451000 \\ \mathrm{C} & 5.76140400 & -1.49094800 & 4.07039400 \\ \mathrm{C} & 6.57228900 & -0.34028200 & 3.82160900 \\ \mathrm{C} & 6.10322500 & 0.26732700 & 2.60435900 \\ \mathrm{C} & 5.01266300 & -0.51706800 & 2.11418900 \\ \mathrm{C} & 4.80039800 & -1.59750000 & 3.02588900 \\ \mathrm{H} & 6.87525700 & 1.70547700 & 7.38339100 \\ \mathrm{H} & 4.76606200 & 2.74530000 & 8.74498600 \\ \mathrm{H} & 3.85432700 & 4.87077000 & 7.33340200 \\ \mathrm{H} & 5.44906300 & 5.20685100 & 5.19032600 \\ \mathrm{H} & 7.30214400 & 3.24582300 & 5.20771800 \\ \mathrm{H} & 1.62476800 & -2.53982500 & 4.80289000 \\ \mathrm{H} & -0.15212000 & -0.66789500 & 4.00543800\end{array}$




$\begin{array}{rrrr}\mathrm{H} & -0.57973100 & 1.02148200 & 6.07512800 \\ \mathrm{H} & 0.94808000 & 0.18067700 & 8.16052800 \\ \mathrm{H} & 2.34369100 & -1.97956200 & 7.33320700 \\ \mathrm{H} & 1.72436500 & 1.69064300 & 1.16285500 \\ \mathrm{H} & 3.78534100 & 3.44486500 & 1.31278000 \\ \mathrm{H} & 3.18129100 & 5.27306300 & 3.21507800 \\ \mathrm{H} & 0.78592000 & 4.62912000 & 4.25864500 \\ \mathrm{H} & -0.14179900 & 2.44831000 & 2.97782500 \\ \mathrm{H} & 5.83344100 & -2.14188600 & 4.93289200 \\ \mathrm{H} & 7.40473300 & -0.00310600 & 4.42512200 \\ \mathrm{H} & 6.52514400 & 1.14153900 & 2.12511300 \\ \mathrm{H} & 4.44108900 & -0.32565300 & 1.21516400 \\ \mathrm{H} & 4.03480900 & -2.35812800 & 2.94097200 \\ \mathrm{H} & 2.71077000 & 0.57834400 & 3.77422900 \\ \mathrm{H} & 4.49227100 & 2.18498400 & 4.02708900\end{array}$

$\mathrm{TS}_{\mathrm{A} 1 / \mathrm{A} 5}$

$\mathrm{E}(\mathrm{RB} 3 \mathrm{PW} 91)=-1306.84131429$ A.U.

Thermal correction to Gibbs Free Energy= 0.319212 A.U.

$\begin{array}{lrrr}\mathrm{Ru} & 4.38757500 & 2.06850400 & 6.21308900 \\ \mathrm{Ru} & 2.19909500 & 0.29363700 & 5.49053100 \\ \mathrm{Ru} & 3.08226400 & 2.41907500 & 3.84646700 \\ \mathrm{Ru} & 4.74446200 & 0.15209500 & 4.26129000 \\ \mathrm{O} & 4.88815900 & 0.09279500 & 6.32250600 \\ \mathrm{O} & 3.69234600 & -0.67095800 & 6.69512300 \\ \mathrm{C} & 6.13375500 & 2.99855400 & 7.17915200 \\ \mathrm{C} & 5.20405500 & 2.59427400 & 8.19439600 \\ \mathrm{C} & 3.98565000 & 3.29952500 & 8.00722400 \\ \mathrm{C} & 4.14639700 & 4.15914000 & 6.86423200 \\ \mathrm{C} & 5.46734900 & 3.96749000 & 6.35798800 \\ \mathrm{C} & 0.68411600 & -1.33009600 & 5.24328800 \\ \mathrm{C} & 0.39324900 & -0.24810300 & 4.35601100 \\ \mathrm{C} & 0.12050000 & 0.91812600 & 5.14202000 \\ \mathrm{C} & 0.23137100 & 0.55632000 & 6.52867100 \\ \mathrm{C} & 0.60185300 & -0.81438700 & 6.57688500 \\ \mathrm{C} & 2.73754500 & 2.77719200 & 1.74824900 \\ \mathrm{C} & 3.69924400 & 3.71773000 & 2.24859500 \\ \mathrm{C} & 3.06839100 & 4.49303400 & 3.27713800 \\ \mathrm{C} & 1.71687200 & 4.02923800 & 3.41630500 \\ \mathrm{C} & 1.51290900 & 2.97270300 & 2.46701900 \\ \mathrm{C} & 6.67255200 & 0.10504900 & 3.15028700 \\ \mathrm{C} & 5.58371800 & 0.17774600 & 2.21717100 \\ \mathrm{C} & 4.77277800 & -0.98329200 & 2.39999300 \\ \mathrm{C} & 5.35913100 & -1.79245100 & 3.43317700 \\ \mathrm{C} & 6.52499900 & -1.10210000 & 3.89205200 \\ \mathrm{H} & 7.15422400 & 2.65526100 & 7.07000300 \\ \mathrm{H} & 5.37963900 & 1.83065500 & 8.94242300 \\ \mathrm{H} & 3.09297600 & 3.19848400 & 8.61089400 \\ \mathrm{H} & 3.40052300 & 4.83293500 & 6.46621100 \\ \mathrm{H} & 5.89340100 & 4.46774800 & 5.49757600 \\ \mathrm{H} & 0.91344100 & -2.34994200 & 4.96340400 \\ \mathrm{H} & 0.37642800 & -0.30112700 & 3.27515600 \\ \mathrm{H} & -0.15965500 & 1.88918400 & 4.75931900 \\ \mathrm{H} & 0.07604900 & 1.20773700 & 7.37840200 \\ \mathrm{H} & 0.83135900 & -1.37074300 & 7.47829400 \\ \mathrm{H} & 2.90160900 & 2.05401400 & 0.95916800 \\ \mathrm{H} & 4.72142600 & 3.82758100 & 1.90804800\end{array}$

$\begin{array}{rrrr}\mathrm{H} & 3.52970100 & 5.29244300 & 3.84339200 \\ \mathrm{H} & 0.97820900 & 4.42092100 & 4.10497100 \\ \mathrm{H} & 0.59285100 & 2.42344000 & 2.31061100 \\ \mathrm{H} & 7.46435600 & 0.83324700 & 3.27029300 \\ \mathrm{H} & 5.40905200 & 0.96921100 & 1.50160100 \\ \mathrm{H} & 3.87063500 & -1.21919800 & 1.84967700 \\ \mathrm{H} & 5.00567100 & -2.75595600 & 3.77604500 \\ \mathrm{H} & 7.16224000 & -1.42020900 & 4.70861300 \\ \mathrm{H} & 2.98344600 & 0.53467800 & 3.88001200 \\ \mathrm{H} & 4.80468400 & 1.99581800 & 4.43082200 \\ \mathrm{H} & 3.47122600 & -0.90268500 & 5.36950200 \\ \mathrm{H} & 2.65450500 & 1.99805700 & 5.76094000\end{array}$

A5

$\mathrm{E}(\mathrm{RB} 3 \mathrm{PW} 91)=-1306.94772379$ A.U.

Thermal correction to Gibbs Free Energy= 0.325655 A.U.

\begin{tabular}{|c|c|c|c|}
\hline $\mathrm{Ru}$ & 4.30749800 & & 6.48010400 \\
\hline $\mathrm{Ru}$ & 2.12242000 & 0.36494400 & 5.56427500 \\
\hline $\mathrm{Ru}$ & 3.04716300 & 2.20977600 & 3.78963000 \\
\hline $\mathrm{Ru}$ & 4.83394500 & 0.24541300 & 4.29465000 \\
\hline $\mathrm{O}$ & 5.40123300 & 0.17039300 & 6.17313000 \\
\hline $\mathrm{O}$ & 3.24739300 & 0.19203300 & 7.25372900 \\
\hline $\mathrm{C}$ & 6.07069600 & 2.79458700 & 7.41940400 \\
\hline $\mathrm{C}$ & 5.04585300 & 2.54885000 & 8.38253000 \\
\hline $\mathrm{C}$ & 3.88666600 & 3.31479600 & 8.00175900 \\
\hline $\mathrm{C}$ & 4.21389600 & 4.00804900 & 6.79768500 \\
\hline $\mathrm{C}$ & 5.56093500 & 3.68449200 & 6.43527900 \\
\hline $\mathrm{C}$ & 0.72178000 & -1.13756300 & 4.78121400 \\
\hline $\mathrm{C}$ & 0.42140500 & 0.11647500 & 4.15775300 \\
\hline $\mathrm{C}$ & 0.04984400 & 1.02997700 & 5.19906100 \\
\hline $\mathrm{C}$ & 0.09528800 & 0.34925600 & 6.45331900 \\
\hline $\mathrm{C}$ & 0.52489700 & -0.99344400 & 6.18510600 \\
\hline $\mathrm{C}$ & 2.46063900 & 2.62164600 & 1.75329100 \\
\hline $\mathrm{C}$ & 3.62294900 & 3.39537400 & 2.07813800 \\
\hline $\mathrm{C}$ & 3.27993800 & 4.26242600 & 3.16756400 \\
\hline $\mathrm{C}$ & 1.89309700 & 4.05889900 & 3.48301300 \\
\hline $\mathrm{C}$ & 1.39154800 & 3.05378500 & 2.61170400 \\
\hline $\mathrm{C}$ & 6.60064900 & 0.36032600 & 2.88058600 \\
\hline $\mathrm{C}$ & 5.38458300 & 0.23988900 & 2.12088900 \\
\hline $\mathrm{C}$ & 4.74922000 & -0.98101400 & 2.47270600 \\
\hline $\mathrm{C}$ & 5.57608200 & -1.63650300 & 3.45819500 \\
\hline $\mathrm{C}$ & 6.71079400 & -0.79873100 & 3.69137400 \\
\hline $\mathrm{H}$ & 7.03835800 & 2.31014500 & 7.39437900 \\
\hline $\mathrm{H}$ & 5.12603500 & 1.89905900 & 9.24480300 \\
\hline $\mathrm{H}$ & 2.94951600 & 3.36535800 & 8.54069500 \\
\hline $\mathrm{H}$ & 3.55109700 & 4.65693900 & 6.24165700 \\
\hline $\mathrm{H}$ & 6.09111300 & 4.03671100 & 5.56036500 \\
\hline $\mathrm{H}$ & 1.04997700 & -2.03713000 & 4.27662600 \\
\hline $\mathrm{H}$ & 0.44027000 & 0.31607300 & 3.09667900 \\
\hline $\mathrm{H}$ & -0.22119300 & 2.06801400 & 5.05803900 \\
\hline $\mathrm{H}$ & -0.14667500 & 0.76359000 & 7.42304800 \\
\hline $\mathrm{H}$ & 0.71366400 & -1.75840400 & 6.92819100 \\
\hline $\mathrm{H}$ & 2.38217400 & 1.87973500 & 0.96800800 \\
\hline $\mathrm{H}$ & 4.58889700 & 3.34186100 & 1.59265200 \\
\hline $\mathrm{H}$ & 3.94164000 & 4.97881400 & 3.63797300 \\
\hline $\mathrm{H}$ & 1.33171300 & 4.57342500 & 4.25300100 \\
\hline $\mathrm{H}$ & 0.37947100 & 2.66880400 & 2.60308200 \\
\hline
\end{tabular}




$\begin{array}{lrrr}\mathrm{H} & 7.30859600 & 1.17746100 & 2.83396900 \\ \mathrm{H} & 5.01444900 & 0.95747700 & 1.40347600 \\ \mathrm{H} & 3.82319300 & -1.36159100 & 2.06270800 \\ \mathrm{H} & 5.38290500 & -2.59634000 & 3.91868800 \\ \mathrm{H} & 7.48181400 & -0.98024000 & 4.42939400 \\ \mathrm{H} & 3.38185000 & -0.53833700 & 4.82879500 \\ \mathrm{H} & 4.71895100 & 2.00881900 & 4.21781000 \\ \mathrm{H} & 4.00755200 & -0.40211300 & 7.04562100 \\ \mathrm{H} & 2.67135200 & 2.12354000 & 5.59368800\end{array}$

$\mathbf{T S}_{\mathrm{A} 2 / \mathrm{A} 5}$

$\mathrm{E}(\mathrm{RB} 3 \mathrm{PW} 91)=-1306.82263152 \mathrm{~A} . \mathrm{U}$.

Thermal correction to Gibbs Free Energy= 0.319193 A.U.

\begin{tabular}{|c|c|c|c|}
\hline $\mathrm{Ru}$ & 4.03509700 & 2.14462900 & 6.70519400 \\
\hline $\mathrm{Ru}$ & 2.37486800 & 0.31927500 & 5.30448600 \\
\hline $\mathrm{Ru}$ & 3.46340100 & 2.53777200 & 3.81597200 \\
\hline $\mathrm{Ru}$ & 4.85746000 & 0.14358400 & 3.84622200 \\
\hline $\mathrm{O}$ & 5.23529300 & 0.66121700 & 5.87088500 \\
\hline $\mathrm{O}$ & 4.14578600 & -0.13184100 & 6.51493000 \\
\hline $\mathrm{C}$ & 5.45723000 & 3.44838900 & 7.70920300 \\
\hline $\mathrm{C}$ & 4.66871400 & 2.77743500 & 8.68371100 \\
\hline $\mathrm{C}$ & 3.28429900 & 3.13346000 & 8.46954900 \\
\hline $\mathrm{C}$ & 3.23146000 & 4.02637300 & 7.36107800 \\
\hline $\mathrm{C}$ & 4.57471100 & 4.20591700 & 6.86702100 \\
\hline $\mathrm{C}$ & 1.11196300 & -1.46538100 & 5.24646000 \\
\hline $\mathrm{C}$ & 0.72297800 & -0.54784800 & 4.21639300 \\
\hline $\mathrm{C}$ & 0.29140000 & 0.66759200 & 4.84025500 \\
\hline $\mathrm{C}$ & 0.39178300 & 0.49513200 & 6.26544600 \\
\hline $\mathrm{C}$ & 0.90857400 & -0.80652100 & 6.50671100 \\
\hline $\mathrm{C}$ & 2.05652800 & 3.04846000 & 2.28779800 \\
\hline $\mathrm{C}$ & 3.38684900 & 3.24832300 & 1.78235200 \\
\hline $\mathrm{C}$ & 4.01407200 & 4.24153700 & 2.60365700 \\
\hline $\mathrm{C}$ & 3.06303000 & 4.66949100 & 3.58841800 \\
\hline $\mathrm{C}$ & 1.84446200 & 3.95186700 & 3.38298600 \\
\hline $\mathrm{C}$ & 6.59922200 & -0.27697600 & 2.58035900 \\
\hline $\mathrm{C}$ & 5.38885800 & -0.41282400 & 1.81080100 \\
\hline $\mathrm{C}$ & 4.62271100 & -1.46338900 & 2.40205500 \\
\hline $\mathrm{C}$ & 5.35832000 & -2.00227100 & 3.51355000 \\
\hline $\mathrm{C}$ & 6.56825200 & -1.25273900 & 3.62024300 \\
\hline $\mathrm{H}$ & 6.52881300 & 3.35579300 & 7.58304600 \\
\hline $\mathrm{H}$ & 5.03834600 & 2.09356000 & 9.43841500 \\
\hline $\mathrm{H}$ & 2.44119900 & 2.79469800 & 9.05846200 \\
\hline $\mathrm{H}$ & 2.33727400 & 4.47162700 & 6.94467700 \\
\hline $\mathrm{H}$ & 4.87451200 & 4.82862600 & 6.03622400 \\
\hline $\mathrm{H}$ & 1.46662500 & -2.47785800 & 5.10331600 \\
\hline $\mathrm{H}$ & 0.75564400 & -0.73645100 & 3.15091900 \\
\hline $\mathrm{H}$ & -0.07371100 & 1.54811100 & 4.32945300 \\
\hline $\mathrm{H}$ & 0.14337600 & 1.23220300 & 7.01765100 \\
\hline $\mathrm{H}$ & 1.14190900 & -1.22249400 & 7.47969600 \\
\hline $\mathrm{H}$ & 1.32710100 & 2.35669800 & 1.88428100 \\
\hline $\mathrm{H}$ & 3.83384900 & 2.73571700 & 0.94098000 \\
\hline $\mathrm{H}$ & 5.02652800 & 4.61061200 & 2.49749900 \\
\hline $\mathrm{H}$ & 3.23409000 & 5.42239800 & 4.34736900 \\
\hline $\mathrm{H}$ & 0.93319600 & 4.06483900 & 3.95610200 \\
\hline $\mathrm{H}$ & 7.39897300 & 0.42917500 & 2.39740000 \\
\hline $\mathrm{H}$ & 5.11690500 & 0.15913100 & 0.93362800 \\
\hline $\mathrm{H}$ & 3.65487200 & -1.80811100 & 2.05976500 \\
\hline
\end{tabular}

$\begin{array}{rrrr}\mathrm{H} & 5.06456400 & -2.83739900 & 4.13604600 \\ \mathrm{H} & 7.31967300 & -1.37521900 & 4.39104100 \\ \mathrm{H} & 3.15491100 & 0.68756600 & 3.66987300 \\ \mathrm{H} & 5.07854500 & 1.83508800 & 3.61433800 \\ \mathrm{H} & 3.94365800 & -0.68205000 & 5.43064300 \\ \mathrm{H} & 2.72015900 & 2.11546700 & 5.44767700\end{array}$

$(\mathrm{CpRu})_{4}(\mathrm{H})_{6}(\mathbf{2 c})$

$\mathrm{E}(\mathrm{RB} 3 \mathrm{PW} 91)=-1157.7370746$ A.U .

Thermal correction to Gibbs Free Energy= 0.332464 A.U.

\begin{tabular}{|c|c|c|c|}
\hline $\mathrm{Ru}$ & 4.26766700 & 2.32755000 & 5.92499000 \\
\hline $\mathrm{Ru}$ & 2.39118000 & 0.43426500 & 5.64823400 \\
\hline $\mathrm{Ru}$ & 2.71849000 & 2.32702300 & 3.44158700 \\
\hline $\mathrm{Ru}$ & 4.59207600 & 0.43121500 & 3.72090800 \\
\hline $\mathrm{C}$ & 5.87776800 & 2.54540800 & 7.37185500 \\
\hline $\mathrm{C}$ & 4.71346300 & 3.21951000 & 7.86585600 \\
\hline $\mathrm{C}$ & 4.37569900 & 4.25708500 & 6.93793700 \\
\hline $\mathrm{C}$ & 5.34533000 & 4.24054800 & 5.88303300 \\
\hline $\mathrm{C}$ & 6.26925900 & 3.18222300 & 6.14768000 \\
\hline $\mathrm{C}$ & 1.42262300 & -1.50594500 & 5.32073300 \\
\hline $\mathrm{C}$ & 0.43938700 & -0.47076800 & 5.24864800 \\
\hline $\mathrm{C}$ & 0.40851500 & 0.20231000 & 6.51467400 \\
\hline $\mathrm{C}$ & 1.36909200 & -0.42706200 & 7.37208300 \\
\hline $\mathrm{C}$ & 2.00657400 & -1.47298700 & 6.62938900 \\
\hline $\mathrm{C}$ & 1.10972300 & 2.54526800 & 1.99313200 \\
\hline $\mathrm{C}$ & 2.27516700 & 3.21758700 & 1.49941300 \\
\hline $\mathrm{C}$ & 2.61336700 & 4.25565200 & 2.42661100 \\
\hline $\mathrm{C}$ & 1.64289400 & 4.24121500 & 3.48075800 \\
\hline $\mathrm{C}$ & 0.71799500 & 3.18364500 & 3.21641800 \\
\hline $\mathrm{C}$ & 5.55754600 & -1.51018700 & 4.05031500 \\
\hline $\mathrm{C}$ & 6.54258800 & -0.47657600 & 4.12047300 \\
\hline $\mathrm{C}$ & 6.57385900 & 0.19478000 & 2.85354700 \\
\hline $\mathrm{C}$ & 5.61175100 & -0.43409800 & 1.99750400 \\
\hline $\mathrm{C}$ & 4.97293500 & -1.47794800 & 2.74194200 \\
\hline $\mathrm{H}$ & 6.38242700 & 1.71386100 & 7.84737800 \\
\hline $\mathrm{H}$ & 4.18499100 & 2.98963300 & 8.78238300 \\
\hline $\mathrm{H}$ & 3.54242000 & 4.94255100 & 7.02618500 \\
\hline $\mathrm{H}$ & 5.36221000 & 4.90151500 & 5.02629800 \\
\hline $\mathrm{H}$ & 7.11344200 & 2.90607200 & 5.52933000 \\
\hline $\mathrm{H}$ & 1.69292200 & -2.18427000 & 4.52209400 \\
\hline $\mathrm{H}$ & -0.16833500 & -0.23011000 & 4.38614600 \\
\hline $\mathrm{H}$ & -0.23776800 & 1.02895900 & 6.78159500 \\
\hline $\mathrm{H}$ & 1.57309900 & -0.16316600 & 8.40207300 \\
\hline $\mathrm{H}$ & 2.78210300 & -2.13256100 & 6.99768700 \\
\hline $\mathrm{H}$ & 0.60449100 & 1.71385400 & 1.51798500 \\
\hline $\mathrm{H}$ & 2.80412800 & 2.98621100 & 0.58354600 \\
\hline $\mathrm{H}$ & 3.44751300 & 4.94006500 & 2.33836700 \\
\hline $\mathrm{H}$ & 1.62610800 & 4.90301100 & 4.33685300 \\
\hline $\mathrm{H}$ & -0.12704800 & 2.90910800 & 3.83431700 \\
\hline $\mathrm{H}$ & 5.28662500 & -2.18707100 & 4.84996700 \\
\hline $\mathrm{H}$ & 7.15126900 & -0.23583500 & 4.98227700 \\
\hline $\mathrm{H}$ & 7.22139800 & 1.01997200 & 2.58517000 \\
\hline $\mathrm{H}$ & 5.40757200 & -0.17120600 & 0.96729300 \\
\hline $\mathrm{H}$ & 4.19606800 & -2.13667400 & 2.37495000 \\
\hline $\mathrm{H}$ & 2.70802600 & 0.48939300 & 3.85208000 \\
\hline $\mathrm{H}$ & 4.47726500 & 2.28703000 & 4.11869000 \\
\hline $\mathrm{H}$ & 4.27599300 & 0.48946500 & 5.51654900 \\
\hline
\end{tabular}




$\begin{array}{llll}\mathrm{H} & 2.50869800 & 2.28899100 & 5.24763600 \\ \mathrm{H} & 3.16338500 & 1.38655300 & 6.86526000 \\ \mathrm{H} & 3.82173000 & 1.38355600 & 2.50282100\end{array}$

B1

$\mathrm{E}(\mathrm{RB} 3 \mathrm{PW} 91)=-1308.05173729$ A.U.

Thermal correction to Gibbs Free Energy= 0.337236 A.U.

\begin{tabular}{|c|c|c|c|}
\hline $\mathrm{Ru}$ & 4.13833600 & 2.38395300 & 6.40354700 \\
\hline $\mathrm{Ru}$ & 2.46663500 & 0.21836600 & 5.55315600 \\
\hline $\mathrm{Ru}$ & 3.25116900 & 2.55136600 & 3.70483300 \\
\hline $\mathrm{Ru}$ & 4.69555900 & 0.19943500 & 3.59764400 \\
\hline $\mathrm{O}$ & 5.10145300 & 0.64377400 & 5.63254100 \\
\hline $\mathrm{O}$ & 4.32274800 & -0.40283200 & 6.25676100 \\
\hline $\mathrm{C}$ & 5.43170400 & 2.62533400 & 8.17936100 \\
\hline $\mathrm{C}$ & 4.07573600 & 2.67586500 & 8.60064900 \\
\hline $\mathrm{C}$ & 3.43623800 & 3.78922900 & 7.94535000 \\
\hline C & 4.41144000 & 4.41162300 & 7.11474700 \\
\hline $\mathrm{C}$ & 5.65583700 & 3.69398100 & 7.25452900 \\
\hline $\mathrm{C}$ & 1.27467100 & -1.44722500 & 6.41495900 \\
\hline $\mathrm{C}$ & 1.02965700 & -1.38075800 & 5.01376200 \\
\hline $\mathrm{C}$ & 0.45039200 & -0.09706700 & 4.71651200 \\
\hline $\mathrm{C}$ & 0.35506400 & 0.62681000 & 5.94218600 \\
\hline $\mathrm{C}$ & 0.86397000 & -0.21194600 & 6.99834300 \\
\hline $\mathrm{C}$ & 2.03476500 & 2.94026100 & 1.88915400 \\
\hline $\mathrm{C}$ & 3.27114600 & 3.65763000 & 1.82006500 \\
\hline $\mathrm{C}$ & 3.31736800 & 4.57200600 & 2.92390300 \\
\hline $\mathrm{C}$ & 2.10471600 & 4.42304800 & 3.67553500 \\
\hline $\mathrm{C}$ & 1.32712800 & 3.40256100 & 3.04360500 \\
\hline $\mathrm{C}$ & 6.67105700 & -0.19337400 & 2.72913000 \\
\hline $\mathrm{C}$ & 5.67407400 & -0.10047300 & 1.69336300 \\
\hline $\mathrm{C}$ & 4.74209000 & -1.16823400 & 1.87835800 \\
\hline $\mathrm{C}$ & 5.16893900 & -1.92397600 & 3.02652200 \\
\hline $\mathrm{C}$ & 6.34785700 & -1.32983900 & 3.53628100 \\
\hline $\mathrm{H}$ & 6.15137600 & 1.86624900 & 8.45864800 \\
\hline $\mathrm{H}$ & 3.60782600 & 1.99104700 & 9.29654200 \\
\hline $\mathrm{H}$ & 2.41026900 & 4.10661800 & 8.08021500 \\
\hline $\mathrm{H}$ & 4.25145900 & 5.28657700 & 6.49749300 \\
\hline $\mathrm{H}$ & 6.59342000 & 3.93244500 & 6.76958600 \\
\hline $\mathrm{H}$ & 1.73784800 & -2.27409800 & 6.93892300 \\
\hline $\mathrm{H}$ & 1.23221100 & -2.16758200 & 4.29829800 \\
\hline $\mathrm{H}$ & 0.12844600 & 0.24549100 & 3.74229900 \\
\hline $\mathrm{H}$ & -0.04780300 & 1.62412500 & 6.06514100 \\
\hline $\mathrm{H}$ & 0.90516900 & 0.03743500 & 8.05079600 \\
\hline $\mathrm{H}$ & 1.69456900 & 2.18536500 & 1.19201300 \\
\hline $\mathrm{H}$ & 4.03471800 & 3.54041600 & 1.06173800 \\
\hline $\mathrm{H}$ & 4.11986600 & 5.26724700 & 3.13461400 \\
\hline $\mathrm{H}$ & 1.82810000 & 4.97660600 & 4.56307900 \\
\hline $\mathrm{H}$ & 0.35905900 & 3.05006500 & 3.37624900 \\
\hline $\mathrm{H}$ & 7.52558300 & 0.45807100 & 2.85766300 \\
\hline $\mathrm{H}$ & 5.64962400 & 0.63542000 & 0.89959100 \\
\hline $\mathrm{H}$ & 3.89052700 & -1.39017400 & 1.24820000 \\
\hline $\mathrm{H}$ & 4.66384700 & -2.78337700 & 3.44843500 \\
\hline $\mathrm{H}$ & 6.85897500 & -1.62838400 & 4.44271400 \\
\hline $\mathrm{H}$ & 3.33819600 & 0.95224500 & 2.92210400 \\
\hline $\mathrm{H}$ & 4.89343200 & 1.89523100 & 3.46024300 \\
\hline $\mathrm{H}$ & 3.24361300 & -0.51186000 & 4.19672500 \\
\hline $\mathrm{H}$ & 2.96775200 & 1.22469800 & 6.88980900 \\
\hline
\end{tabular}

$\begin{array}{llll}\mathrm{H} & 2.66565200 & 2.11230400 & 5.34619900 \\ \mathrm{H} & 4.50214400 & 3.00895000 & 4.83644700\end{array}$

\section{$\mathrm{TS}_{\mathrm{B} 1 / \mathrm{B} 2}$}

$\mathrm{E}(\mathrm{RB} 3 \mathrm{PW} 91)=-1308.03511086$ A.U.

Thermal correction to Gibbs Free Energy= 0.336269 A.U.

$\begin{array}{llll}\mathrm{Ru} & 4.16810400 & 2.42711000 & 6.44731100\end{array}$

$\begin{array}{llll}\mathrm{Ru} & 2.45920600 & 0.25956600 & 5.49080800\end{array}$

$\begin{array}{llll}\mathrm{Ru} & 3.28880900 & 2.62660800 & 3.80209900\end{array}$

$\begin{array}{llll}\mathrm{Ru} & 4.67467500 & 0.19072100 & 3.67913000\end{array}$

$\begin{array}{llll}\mathrm{O} & 5.15369400 & 0.69682000 & 5.71304200\end{array}$

$\begin{array}{llll}\text { O } & 4.34525000 & -0.38854800 & 6.29733600\end{array}$

$\begin{array}{llll}\text { C } & 5.51014800 & 2.55313600 & 8.24581400\end{array}$

$\begin{array}{llll}\text { C } & 4.16179800 & 2.57743000 & 8.68170000\end{array}$

$\begin{array}{llll}\text { C } & 3.52162200 & 3.72283800 & 8.08664300\end{array}$

$\begin{array}{llll}\text { C } & 4.49539500 & 4.39630900 & 7.28626400\end{array}$

$\begin{array}{llll}\text { C } & 5.73383400 & 3.66761000 & 7.37987900\end{array}$

$\begin{array}{llll}\text { C } & 1.23225500 & -1.35838400 & 6.36222200\end{array}$

$\begin{array}{llll}\text { C } & 1.16034400 & -1.44889400 & 4.93709800\end{array}$

$\begin{array}{llll}\text { C } & 0.59870500 & -0.21813800 & 4.45782600\end{array}$

$\begin{array}{llll}\text { C } & 0.30877600 & 0.61673700 & 5.58298700\end{array}$

$\begin{array}{llll}\text { C } & 0.69978000 & -0.10089300 & 6.76831200\end{array}$

$\begin{array}{llll}\text { C } & 2.40009700 & 2.88909700 & 1.81281500\end{array}$

$\begin{array}{llll}\text { C } & 3.52022600 & 3.77023800 & 1.97039800\end{array}$

$\begin{array}{llll}\text { C } & 3.23007000 & 4.67830900 & 3.04067200\end{array}$

$\begin{array}{llll}\text { C } & 1.93093100 & 4.35953200 & 3.54837900\end{array}$

$\begin{array}{llll}\text { C } & 1.42850900 & 3.24892100 & 2.80138000\end{array}$

$\begin{array}{llll}\text { C } & 6.36320400 & -0.12888400 & 2.31844700\end{array}$

$\begin{array}{llll}\text { C } & 5.11855600 & -0.22065000 & 1.59206400\end{array}$

$\begin{array}{llll}\text { C } & 4.37734900 & -1.31514500 & 2.12277100\end{array}$

$\begin{array}{llll}\text { C } & 5.15962500 & -1.91496400 & 3.17289800\end{array}$

$\begin{array}{llll}\text { C } & 6.37348500 & -1.17709000 & 3.28644800\end{array}$

$\begin{array}{llll}\mathrm{H} & 6.22670900 & 1.77144700 & 8.46529800\end{array}$

$\begin{array}{llll}\mathrm{H} & 3.69587600 & 1.85083900 & 9.33474400\end{array}$

$\begin{array}{llll}\mathrm{H} & 2.49881200 & 4.03791600 & 8.24842000\end{array}$

$\begin{array}{llll}\mathrm{H} & 4.33656800 & 5.30809200 & 6.72455100\end{array}$

$\begin{array}{llll}\mathrm{H} & 6.66940000 & 3.92599700 & 6.90133200\end{array}$

$\begin{array}{llll}\mathrm{H} & 1.64937800 & -2.10880500 & 7.02327000\end{array}$

$\begin{array}{llll}\mathrm{H} & 1.46607800 & -2.29321400 & 4.33339500\end{array}$

$\begin{array}{llll}\mathrm{H} & 0.41283200 & 0.03004200 & 3.42069900\end{array}$

$\mathrm{H} \quad-0.14974500 \quad 1.59635100 \quad 5.55193000$

$\begin{array}{llll}\mathrm{H} & 0.60192100 & 0.24803200 & 7.78830400\end{array}$

$\begin{array}{llll}\mathrm{H} & 2.30141100 & 2.10229200 & 1.07666100\end{array}$

$\begin{array}{llll}\mathrm{H} & 4.42576500 & 3.76452700 & 1.37714800\end{array}$

$\begin{array}{llll}\mathrm{H} & 3.87662200 & 5.47212900 & 3.39195600\end{array}$

$\begin{array}{llll}\mathrm{H} & 1.42022300 & 4.86705400 & 4.35669500\end{array}$

$\begin{array}{llll}\mathrm{H} & 0.46830300 & 2.77171700 & 2.95038900\end{array}$

$\begin{array}{llll}\mathrm{H} & 7.15646300 & 0.58707700 & 2.14733800\end{array}$

$\begin{array}{llll}\mathrm{H} & 4.80648500 & 0.42121100 & 0.77847600\end{array}$

$\mathrm{H} \quad 3.40491700 \quad-1.64877100 \quad 1.78351400$

$\begin{array}{llll}\mathrm{H} & 4.88953700 & -2.78471900 & 3.75792400\end{array}$

$\mathrm{H} \quad 7.14902500 \quad-1.35172700 \quad 4.02196500$

$\begin{array}{llll}\mathrm{H} & 2.98804500 & 0.80619800 & 3.79260100\end{array}$

$\begin{array}{llll}\mathrm{H} & 4.83649800 & 1.89172900 & 3.43962000\end{array}$

$\begin{array}{llll}\mathrm{H} & 3.99949500 & -0.77481100 & 4.96259100\end{array}$

$\begin{array}{llll}\mathrm{H} & 2.80972400 & 1.40084000 & 6.73030800\end{array}$

$\begin{array}{llll}\mathrm{H} & 2.83662100 & 2.90217500 & 5.48329200\end{array}$ 
B2

$\mathrm{E}(\mathrm{RB} 3 \mathrm{PW} 91)=-1308.06670676$ A.U.

Thermal correction to Gibbs Free Energy= 0.338301 A.U.

\begin{tabular}{|c|c|c|c|}
\hline $\mathrm{Ru}$ & 4.16239400 & 2.37967900 & 6.50304800 \\
\hline $\mathrm{Ru}$ & 2.39208200 & 0.29281800 & 5.51715700 \\
\hline $\mathrm{Ru}$ & 3.35561300 & 2.59641000 & 3.79006200 \\
\hline $\mathrm{Ru}$ & 4.72356300 & 0.21091200 & 3.63525000 \\
\hline $\mathrm{O}$ & 5.15905100 & 0.64618000 & 5.72205800 \\
\hline $\mathrm{O}$ & 4.33579000 & -0.46206400 & 6.29099300 \\
\hline $\mathrm{C}$ & 5.61008600 & 2.72175300 & 8.16964600 \\
\hline $\mathrm{C}$ & 4.30515400 & 2.55791600 & 8.70979300 \\
\hline $\mathrm{C}$ & 3.47123900 & 3.59861600 & 8.15932800 \\
\hline $\mathrm{C}$ & 4.27827400 & 4.39331700 & 7.28960100 \\
\hline $\mathrm{C}$ & 5.61219900 & 3.85009300 & 7.29731000 \\
\hline $\mathrm{C}$ & 1.14207900 & -1.30782900 & 6.31965700 \\
\hline $\mathrm{C}$ & 1.12676600 & -1.37641700 & 4.88404700 \\
\hline $\mathrm{C}$ & 0.59421700 & -0.13372900 & 4.41063100 \\
\hline $\mathrm{C}$ & 0.27013200 & 0.68910000 & 5.54061000 \\
\hline $\mathrm{C}$ & 0.59787600 & -0.05598400 & 6.72706800 \\
\hline $\mathrm{C}$ & 2.47769400 & 2.92866700 & 1.80621800 \\
\hline $\mathrm{C}$ & 3.59002400 & 3.81099900 & 1.99573900 \\
\hline $\mathrm{C}$ & 3.29394200 & 4.68222100 & 3.09407600 \\
\hline $\mathrm{C}$ & 1.99468400 & 4.34385400 & 3.58518400 \\
\hline $\mathrm{C}$ & 1.50035700 & 3.25462300 & 2.80431800 \\
\hline $\mathrm{C}$ & 6.33099700 & -0.18588200 & 2.23989300 \\
\hline $\mathrm{C}$ & 5.05698700 & -0.27743700 & 1.56723300 \\
\hline $\mathrm{C}$ & 4.31870500 & -1.33105300 & 2.18672700 \\
\hline $\mathrm{C}$ & 5.13205700 & -1.92186600 & 3.21882200 \\
\hline $\mathrm{C}$ & 6.36532000 & -1.19361800 & 3.24948800 \\
\hline $\mathrm{H}$ & 6.44788500 & 2.05614900 & 8.33803300 \\
\hline $\mathrm{H}$ & 3.99921200 & 1.79205000 & 9.41053100 \\
\hline $\mathrm{H}$ & 2.42699300 & 3.76793200 & 8.38931100 \\
\hline $\mathrm{H}$ & 3.95031300 & 5.26511100 & 6.73816000 \\
\hline $\mathrm{H}$ & 6.46411000 & 4.23024300 & 6.74891300 \\
\hline $\mathrm{H}$ & 1.51575100 & -2.07698200 & 6.98580400 \\
\hline $\mathrm{H}$ & 1.43934400 & -2.21641600 & 4.27797500 \\
\hline $\mathrm{H}$ & 0.46133900 & 0.14075000 & 3.37198100 \\
\hline $\mathrm{H}$ & -0.17379900 & 1.67545000 & 5.50673700 \\
\hline $\mathrm{H}$ & 0.46708100 & 0.27818900 & 7.74835900 \\
\hline $\mathrm{H}$ & 2.38421500 & 2.16947100 & 1.04112100 \\
\hline $\mathrm{H}$ & 4.49984300 & 3.82503000 & 1.40907000 \\
\hline $\mathrm{H}$ & 3.93628000 & 5.46726800 & 3.47166200 \\
\hline $\mathrm{H}$ & 1.48178000 & 4.81908400 & 4.41153900 \\
\hline $\mathrm{H}$ & 0.54001000 & 2.77173400 & 2.93329600 \\
\hline $\mathrm{H}$ & 7.12650400 & 0.51252800 & 2.01373500 \\
\hline $\mathrm{H}$ & 4.72701700 & 0.33013500 & 0.73479700 \\
\hline $\mathrm{H}$ & 3.32095500 & -1.64857400 & 1.90941000 \\
\hline $\mathrm{H}$ & 4.88570700 & -2.79835700 & 3.80693800 \\
\hline $\mathrm{H}$ & 7.18202600 & -1.35841600 & 3.94266100 \\
\hline $\mathrm{H}$ & 3.00518700 & 0.78107400 & 3.84001500 \\
\hline $\mathrm{H}$ & 4.91465000 & 1.90788300 & 3.38522400 \\
\hline $\mathrm{H}$ & 4.58232400 & -1.16987200 & 5.66739000 \\
\hline $\mathrm{H}$ & 2.86263900 & 1.31388800 & 6.85661500 \\
\hline $\mathrm{H}$ & 2.77917800 & 2.61018600 & 5.43588000 \\
\hline $\mathrm{H}$ & 4.62620000 & 2.96223200 & 4.93744200 \\
\hline
\end{tabular}

$\mathrm{TS}_{\mathrm{B} 2 / \mathrm{B} 3}$

$\mathrm{E}(\mathrm{RB} 3 \mathrm{PW} 91)=-1308.05932482$ A.U.

Thermal correction to Gibbs Free Energy= 0.338444 A.U.

\begin{tabular}{|c|c|c|c|}
\hline $\mathrm{Ru}$ & 4.17926100 & 2.40125500 & 6.42761100 \\
\hline $\mathrm{Ru}$ & 2.40899500 & 0.26367500 & 5.49539100 \\
\hline $\mathrm{Ru}$ & 3.25900400 & 2.63266900 & 3.79295400 \\
\hline $\mathrm{Ru}$ & 4.70543100 & 0.27030000 & 3.69913900 \\
\hline $\mathrm{O}$ & 5.15062400 & 0.74710600 & 5.59663100 \\
\hline $\mathrm{O}$ & 4.22614600 & -0.52223600 & 6.40536900 \\
\hline $\mathrm{C}$ & 5.60003000 & 2.54556900 & 8.15482100 \\
\hline $\mathrm{C}$ & 4.27427600 & 2.51401400 & 8.65996800 \\
\hline $\mathrm{C}$ & 3.57081900 & 3.65166200 & 8.12652600 \\
\hline $\mathrm{C}$ & 4.47864400 & 4.37578800 & 7.29752000 \\
\hline $\mathrm{C}$ & 5.74416900 & 3.68985900 & 7.31170800 \\
\hline $\mathrm{C}$ & 1.12249400 & -1.32868600 & 6.29596300 \\
\hline $\mathrm{C}$ & 1.09569100 & -1.38085400 & 4.86308800 \\
\hline $\mathrm{C}$ & 0.58486300 & -0.11957900 & 4.40795500 \\
\hline $\mathrm{C}$ & 0.28459900 & 0.69314700 & 5.55050500 \\
\hline $\mathrm{C}$ & 0.60832200 & -0.07297900 & 6.72469900 \\
\hline $\mathrm{C}$ & 2.37373300 & 2.89394400 & 1.80507700 \\
\hline $\mathrm{C}$ & 3.48463000 & 3.78486300 & 1.96800400 \\
\hline $\mathrm{C}$ & 3.18570500 & 4.68706800 & 3.04246300 \\
\hline $\mathrm{C}$ & 1.88820200 & 4.35896200 & 3.54482700 \\
\hline $\mathrm{C}$ & 1.39958900 & 3.24325000 & 2.79696400 \\
\hline $\mathrm{C}$ & 6.38441900 & -0.10795000 & 2.34078900 \\
\hline $\mathrm{C}$ & 5.15426300 & -0.25450900 & 1.60103600 \\
\hline $\mathrm{C}$ & 4.43095900 & -1.33366000 & 2.17385300 \\
\hline $\mathrm{C}$ & 5.19969100 & -1.88180500 & 3.25397400 \\
\hline $\mathrm{C}$ & 6.40195600 & -1.11244700 & 3.35327100 \\
\hline $\mathrm{H}$ & 6.35507500 & 1.78798400 & 8.32381100 \\
\hline $\mathrm{H}$ & 3.86546900 & 1.75914900 & 9.31899100 \\
\hline $\mathrm{H}$ & 2.54427900 & 3.92314200 & 8.33707200 \\
\hline $\mathrm{H}$ & 4.25889700 & 5.29168900 & 6.76376300 \\
\hline $\mathrm{H}$ & 6.64552900 & 3.99371200 & 6.79579200 \\
\hline $\mathrm{H}$ & 1.49997200 & -2.10721200 & 6.94867800 \\
\hline $\mathrm{H}$ & 1.38479900 & -2.22093500 & 4.24566700 \\
\hline $\mathrm{H}$ & 0.43959500 & 0.16571100 & 3.37384000 \\
\hline $\mathrm{H}$ & -0.14630400 & 1.68588400 & 5.53418100 \\
\hline $\mathrm{H}$ & 0.49216900 & 0.24942200 & 7.75150900 \\
\hline $\mathrm{H}$ & 2.28165300 & 2.11026200 & 1.06482100 \\
\hline $\mathrm{H}$ & 4.39223500 & 3.78822600 & 1.37785200 \\
\hline $\mathrm{H}$ & 3.82538100 & 5.48528700 & 3.39638400 \\
\hline $\mathrm{H}$ & 1.37291500 & 4.85730400 & 4.35591800 \\
\hline $\mathrm{H}$ & 0.44457700 & 2.755666700 & 2.94651400 \\
\hline $\mathrm{H}$ & 7.16895300 & 0.61083700 & 2.14242800 \\
\hline $\mathrm{H}$ & 4.84432800 & 0.34253300 & 0.75328800 \\
\hline $\mathrm{H}$ & 3.45845100 & -1.68386200 & 1.85016600 \\
\hline $\mathrm{H}$ & 4.94100100 & -2.75332000 & 3.84329500 \\
\hline $\mathrm{H}$ & 7.18789800 & -1.25341000 & 4.08577900 \\
\hline $\mathrm{H}$ & 2.99580600 & 0.75615100 & 3.83850700 \\
\hline $\mathrm{H}$ & 4.82708300 & 1.92084100 & 3.35293200 \\
\hline $\mathrm{H}$ & 4.56376600 & -1.20817900 & 5.81323300 \\
\hline $\mathrm{H}$ & 2.83984200 & 1.35720700 & 6.76065700 \\
\hline $\mathrm{H}$ & 2.79244200 & 2.81197600 & 5.46657300 \\
\hline $\mathrm{H}$ & 4.57769800 & 3.05455400 & 4.89480000 \\
\hline
\end{tabular}


B3

$\mathrm{E}(\mathrm{RB} 3 \mathrm{PW} 91)=-1308.1360604$ A.U.

Thermal correction to Gibbs Free Energy= 0.340586 A.U.

\begin{tabular}{|c|c|c|c|}
\hline $\mathrm{Ru}$ & 4.08288400 & 2.56336200 & 6.35934200 \\
\hline $\mathrm{Ru}$ & 2.63274200 & 0.29622300 & 5.70876100 \\
\hline $\mathrm{Ru}$ & 3.06678400 & 2.57557700 & 3.76375300 \\
\hline $\mathrm{Ru}$ & 4.51669300 & 0.23151500 & 3.67627100 \\
\hline $\mathrm{O}$ & 4.59102300 & 0.69871400 & 5.61552600 \\
\hline $\mathrm{O}$ & 3.30689300 & -0.37294900 & 7.55293300 \\
\hline $\mathrm{C}$ & 5.59599300 & 2.50662600 & 8.07420800 \\
\hline $\mathrm{C}$ & 4.30332600 & 2.69795400 & 8.62126100 \\
\hline $\mathrm{C}$ & 3.76884900 & 3.90996400 & 8.07397300 \\
\hline $\mathrm{C}$ & 4.74700500 & 4.45960700 & 7.18203800 \\
\hline $\mathrm{C}$ & 5.88598000 & 3.58125000 & 7.18128300 \\
\hline $\mathrm{C}$ & 1.64761300 & -1.67335100 & 5.49460200 \\
\hline $\mathrm{C}$ & 1.56167400 & -1.03364400 & 4.23063400 \\
\hline $\mathrm{C}$ & 0.79332600 & 0.17188400 & 4.38316700 \\
\hline $\mathrm{C}$ & 0.39368500 & 0.26668600 & 5.75050100 \\
\hline $\mathrm{C}$ & 0.92601200 & -0.85465900 & 6.43513400 \\
\hline $\mathrm{C}$ & 1.72768800 & 2.84305300 & 2.03889600 \\
\hline $\mathrm{C}$ & 3.00912100 & 3.43119600 & 1.77873200 \\
\hline $\mathrm{C}$ & 3.22341400 & 4.47892200 & 2.73299900 \\
\hline $\mathrm{C}$ & 2.06564700 & 4.54140800 & 3.57875300 \\
\hline $\mathrm{C}$ & 1.15500600 & 3.52808500 & 3.16148800 \\
\hline $\mathrm{C}$ & 6.46273000 & -0.23341900 & 2.82575300 \\
\hline $\mathrm{C}$ & 5.46932600 & -0.21397100 & 1.78190800 \\
\hline $\mathrm{C}$ & 4.54464300 & -1.27193300 & 2.03944300 \\
\hline $\mathrm{C}$ & 4.97117200 & -1.95604200 & 3.22424400 \\
\hline $\mathrm{C}$ & 6.14636200 & -1.32154900 & 3.70081100 \\
\hline $\mathrm{H}$ & 6.22490800 & 1.64139800 & 8.24372500 \\
\hline $\mathrm{H}$ & 3.78918000 & 2.00810800 & 9.27736500 \\
\hline $\mathrm{H}$ & 2.80915900 & 4.34783600 & 8.31595400 \\
\hline $\mathrm{H}$ & 4.66478600 & 5.39344500 & 6.64049100 \\
\hline $\mathrm{H}$ & 6.80490600 & 3.72477200 & 6.62813100 \\
\hline $\mathrm{H}$ & 2.17105700 & -2.58998400 & 5.72729300 \\
\hline $\mathrm{H}$ & 1.96970800 & -1.39871900 & 3.30039100 \\
\hline $\mathrm{H}$ & 0.51976500 & 0.84848300 & 3.58986200 \\
\hline $\mathrm{H}$ & -0.18295000 & 1.06802900 & 6.19339300 \\
\hline $\mathrm{H}$ & 0.87375000 & -1.03059200 & 7.50060300 \\
\hline $\mathrm{H}$ & 1.26460500 & 2.04930200 & 1.46588100 \\
\hline $\mathrm{H}$ & 3.69353900 & 3.14221100 & 0.99137600 \\
\hline $\mathrm{H}$ & 4.09110800 & 5.12362600 & 2.78754000 \\
\hline $\mathrm{H}$ & 1.91206300 & 5.23254700 & 4.39764300 \\
\hline $\mathrm{H}$ & 0.19108500 & 3.32493700 & 3.61170900 \\
\hline $\mathrm{H}$ & 7.31943900 & 0.42319000 & 2.90415100 \\
\hline $\mathrm{H}$ & 5.44841000 & 0.46277300 & 0.93736900 \\
\hline $\mathrm{H}$ & 3.69454200 & -1.53452500 & 1.42202200 \\
\hline $\mathrm{H}$ & 4.47382600 & -2.79725100 & 3.69071400 \\
\hline $\mathrm{H}$ & 6.67613000 & -1.57737600 & 4.61004000 \\
\hline $\mathrm{H}$ & 3.15550800 & 0.88365000 & 2.99021600 \\
\hline $\mathrm{H}$ & 4.71648400 & 1.89024300 & 3.43760900 \\
\hline $\mathrm{H}$ & 4.25104600 & -0.41707200 & 7.36526400 \\
\hline $\mathrm{H}$ & 2.56854800 & 1.77054900 & 6.60755800 \\
\hline $\mathrm{H}$ & 2.84088400 & 3.27515800 & 5.61476500 \\
\hline $\mathrm{H}$ & 4.51294500 & 3.00380600 & 4.80907600 \\
\hline
\end{tabular}

$\mathrm{TS}_{\mathrm{B} 3 / \mathrm{B} 4}$

$\mathrm{E}(\mathrm{RB} 3 \mathrm{PW} 91)=-1308.12080651$ A.U.

Thermal correction to Gibbs Free Energy= 0.340216 A.U.

\begin{tabular}{|c|c|c|c|}
\hline $\mathrm{Ru}$ & 4.13438600 & 2.46368500 & 6.42651400 \\
\hline $\mathrm{Ru}$ & 2.61534400 & 0.31537000 & 5.66228100 \\
\hline $\mathrm{Ru}$ & 3.12163000 & 2.55077600 & 3.77900400 \\
\hline $\mathrm{Ru}$ & 4.52954800 & 0.17535800 & 3.64505600 \\
\hline $\mathrm{O}$ & 4.61725700 & 0.58635300 & 5.59492200 \\
\hline $\mathrm{O}$ & 3.23910600 & -0.30231000 & 7.61442300 \\
\hline $\mathrm{C}$ & 5.55619500 & 2.69482800 & 8.11067000 \\
\hline $\mathrm{C}$ & 4.22985300 & 2.88252300 & 8.60651600 \\
\hline $\mathrm{C}$ & 3.65195600 & 4.01308100 & 7.94080800 \\
\hline $\mathrm{C}$ & 4.62130400 & 4.50438300 & 7.01939000 \\
\hline $\mathrm{C}$ & 5.81373500 & 3.69966500 & 7.12948800 \\
\hline $\mathrm{C}$ & 1.62282500 & -1.64411700 & 5.49673300 \\
\hline $\mathrm{C}$ & 1.55769900 & -1.04618700 & 4.21164800 \\
\hline $\mathrm{C}$ & 0.81871500 & 0.17885300 & 4.32729400 \\
\hline $\mathrm{C}$ & 0.38234100 & 0.31922500 & 5.68399100 \\
\hline $\mathrm{C}$ & 0.89353500 & -0.78812000 & 6.40261800 \\
\hline $\mathrm{C}$ & 1.74123600 & 2.95494100 & 2.08750300 \\
\hline $\mathrm{C}$ & 3.03426900 & 3.51614200 & 1.83027500 \\
\hline $\mathrm{C}$ & 3.30670400 & 4.49217000 & 2.84312300 \\
\hline $\mathrm{C}$ & 2.17688900 & 4.53494000 & 3.72911100 \\
\hline $\mathrm{C}$ & 1.22337600 & 3.58060300 & 3.26735300 \\
\hline $\mathrm{C}$ & 6.45456000 & -0.27929300 & 2.75703600 \\
\hline $\mathrm{C}$ & 5.44666900 & -0.23235400 & 1.72775800 \\
\hline $\mathrm{C}$ & 4.51763200 & -1.28774700 & 1.97811700 \\
\hline $\mathrm{C}$ & 4.95869400 & -2.00027600 & 3.14293400 \\
\hline $\mathrm{C}$ & 6.14639900 & -1.38610100 & 3.61224700 \\
\hline $\mathrm{H}$ & 6.23551200 & 1.90706700 & 8.41096100 \\
\hline $\mathrm{H}$ & 3.74927300 & 2.27282000 & 9.36097700 \\
\hline $\mathrm{H}$ & 2.66199300 & 4.41628200 & 8.10859000 \\
\hline $\mathrm{H}$ & 4.49030500 & 5.35221400 & 6.35894200 \\
\hline $\mathrm{H}$ & 6.73752600 & 3.84079700 & 6.58413100 \\
\hline $\mathrm{H}$ & 2.12478700 & -2.56436800 & 5.76165400 \\
\hline $\mathrm{H}$ & 1.97769600 & -1.43917800 & 3.29832200 \\
\hline $\mathrm{H}$ & 0.58810100 & 0.84815900 & 3.51389700 \\
\hline $\mathrm{H}$ & -0.19608300 & 1.13723400 & 6.09205700 \\
\hline $\mathrm{H}$ & 0.82126300 & -0.93772600 & 7.47119100 \\
\hline $\mathrm{H}$ & 1.23935500 & 2.21176100 & 1.48024600 \\
\hline $\mathrm{H}$ & 3.68736400 & 3.25834300 & 1.00633600 \\
\hline $\mathrm{H}$ & 4.19707400 & 5.10382000 & 2.91177600 \\
\hline $\mathrm{H}$ & 2.06910400 & 5.16833800 & 4.59969800 \\
\hline $\mathrm{H}$ & 0.26796400 & 3.37101500 & 3.73277400 \\
\hline $\mathrm{H}$ & 7.31547800 & 0.37150400 & 2.83796100 \\
\hline $\mathrm{H}$ & 5.41645300 & 0.46493900 & 0.90039600 \\
\hline $\mathrm{H}$ & 3.65681000 & -1.53222400 & 1.36807300 \\
\hline $\mathrm{H}$ & 4.46356400 & -2.84869000 & 3.59891100 \\
\hline $\mathrm{H}$ & 6.68795100 & -1.66223700 & 4.50848900 \\
\hline $\mathrm{H}$ & 3.19073500 & 0.90285700 & 2.95889300 \\
\hline $\mathrm{H}$ & 4.74803800 & 1.85042000 & 3.45926400 \\
\hline $\mathrm{H}$ & 4.17426100 & -0.44432000 & 7.41789800 \\
\hline $\mathrm{H}$ & 3.26550000 & 1.30750400 & 7.24973500 \\
\hline $\mathrm{H}$ & 2.61229400 & 2.29648400 & 5.61160400 \\
\hline $\mathrm{H}$ & 4.45584500 & 2.96984300 & 4.79696500 \\
\hline
\end{tabular}


B4

$\mathrm{E}(\mathrm{RB} 3 \mathrm{PW} 91)=-1308.15337830$ A.U.

Thermal correction to Gibbs Free Energy=0.341490 A.U.

\begin{tabular}{|c|c|c|c|}
\hline $\mathrm{Ru}$ & 4.14663900 & 2.51841300 & 6.35349800 \\
\hline $\mathrm{Ru}$ & 2.57279500 & 0.37237300 & 5.65128700 \\
\hline $\mathrm{Ru}$ & 3.17408700 & 2.55974200 & 3.73631700 \\
\hline $\mathrm{Ru}$ & 4.53614100 & 0.13365400 & 3.64380400 \\
\hline $\mathrm{O}$ & 4.57875200 & 0.59883500 & 5.59384400 \\
\hline $\mathrm{O}$ & 3.53183400 & -0.29961800 & 7.70850900 \\
\hline $\mathrm{C}$ & 5.56931400 & 2.79854000 & 8.03274900 \\
\hline $\mathrm{C}$ & 4.24484400 & 2.77555800 & 8.57370200 \\
\hline $\mathrm{C}$ & 3.50952000 & 3.85254500 & 7.96204300 \\
\hline $\mathrm{C}$ & 4.38566100 & 4.54241100 & 7.07598700 \\
\hline $\mathrm{C}$ & 5.67198300 & 3.88572200 & 7.11688500 \\
\hline $\mathrm{C}$ & 1.56351300 & -1.60854400 & 5.54453600 \\
\hline $\mathrm{C}$ & 1.53564900 & -1.04803400 & 4.24647300 \\
\hline $\mathrm{C}$ & 0.83854500 & 0.21159400 & 4.31949300 \\
\hline $\mathrm{C}$ & 0.36529000 & 0.39551200 & 5.66015200 \\
\hline $\mathrm{C}$ & 0.85743000 & -0.69730100 & 6.41826100 \\
\hline $\mathrm{C}$ & 1.80806200 & 2.99872800 & 2.01891700 \\
\hline $\mathrm{C}$ & 3.12555100 & 3.51642300 & 1.79304400 \\
\hline $\mathrm{C}$ & 3.40329800 & 4.48717400 & 2.81307600 \\
\hline $\mathrm{C}$ & 2.25489000 & 4.56267600 & 3.67515500 \\
\hline $\mathrm{C}$ & 1.28958800 & 3.63412900 & 3.18906900 \\
\hline $\mathrm{C}$ & 6.42888500 & -0.32805700 & 2.71485300 \\
\hline $\mathrm{C}$ & 5.39968600 & -0.28133000 & 1.70768000 \\
\hline $\mathrm{C}$ & 4.46510700 & -1.32343700 & 1.98678100 \\
\hline $\mathrm{C}$ & 4.92767300 & -2.03447800 & 3.14721900 \\
\hline $\mathrm{C}$ & 6.13145100 & -1.42898700 & 3.58534500 \\
\hline $\mathrm{H}$ & 6.36224600 & 2.09959400 & 8.27262500 \\
\hline $\mathrm{H}$ & 3.89459300 & 2.14476300 & 9.38439000 \\
\hline $\mathrm{H}$ & 2.47836800 & 4.11220000 & 8.16695200 \\
\hline $\mathrm{H}$ & 4.13945000 & 5.42058900 & 6.49271600 \\
\hline $\mathrm{H}$ & 6.55878100 & 4.17679900 & 6.56896500 \\
\hline $\mathrm{H}$ & 2.02762800 & -2.53943600 & 5.84235900 \\
\hline $\mathrm{H}$ & 1.95727900 & -1.47690600 & 3.35000900 \\
\hline $\mathrm{H}$ & 0.63838900 & 0.86068200 & 3.48121300 \\
\hline $\mathrm{H}$ & -0.21267700 & 1.22966300 & 6.03398900 \\
\hline $\mathrm{H}$ & 0.75264200 & -0.82046000 & 7.48923900 \\
\hline $\mathrm{H}$ & 1.29596600 & 2.26950800 & 1.40351600 \\
\hline $\mathrm{H}$ & 3.78429700 & 3.24843700 & 0.97685900 \\
\hline $\mathrm{H}$ & 4.30645400 & 5.07782000 & 2.89715900 \\
\hline $\mathrm{H}$ & 2.14367300 & 5.19876700 & 4.54272500 \\
\hline $\mathrm{H}$ & 0.32428300 & 3.43942000 & 3.64124200 \\
\hline $\mathrm{H}$ & 7.29647100 & 0.31648500 & 2.77207300 \\
\hline $\mathrm{H}$ & 5.35238000 & 0.41611100 & 0.88123500 \\
\hline $\mathrm{H}$ & 3.58786900 & -1.56253200 & 1.39830000 \\
\hline $\mathrm{H}$ & 4.43696900 & -2.87813700 & 3.61699200 \\
\hline $\mathrm{H}$ & 6.69830900 & -1.71011800 & 4.46443100 \\
\hline $\mathrm{H}$ & 3.19029600 & 0.95276700 & 2.97651900 \\
\hline $\mathrm{H}$ & 4.76172100 & 1.82891800 & 3.43357900 \\
\hline $\mathrm{H}$ & 4.32983000 & -0.31822900 & 7.13227700 \\
\hline $\mathrm{H}$ & 3.58141000 & 0.61268100 & 8.04492600 \\
\hline $\mathrm{H}$ & 2.54612700 & 2.18846500 & 5.73142200 \\
\hline $\mathrm{H}$ & 4.50108900 & 3.03696000 & 4.76215800 \\
\hline
\end{tabular}

$\mathbf{T S}_{\mathbf{B} 4 / 4 \mathrm{~b}}$

$\mathrm{E}(\mathrm{RB} 3 \mathrm{PW} 91)=-1308.15354523$ A.U.

Thermal correction to Gibbs Free Energy $=0.342887$ A.U.

\begin{tabular}{|c|c|c|c|}
\hline $\mathrm{Ru}$ & 4.00409000 & 2.38584500 & 6.39175800 \\
\hline $\mathrm{Ru}$ & 2.63730800 & 0.17841400 & 5.34673300 \\
\hline $\mathrm{Ru}$ & 3.17109300 & 2.62104900 & 3.73450400 \\
\hline $\mathrm{Ru}$ & 4.64716600 & 0.27680900 & 3.51756400 \\
\hline $\mathrm{O}$ & 4.57730100 & 0.63963600 & 5.58824200 \\
\hline $\mathrm{O}$ & 4.06196400 & -1.87391800 & 5.97877500 \\
\hline $\mathrm{C}$ & 5.20627700 & 2.36110100 & 8.28071800 \\
\hline $\mathrm{C}$ & 3.84395600 & 2.47185900 & 8.65158600 \\
\hline $\mathrm{C}$ & 3.31085000 & 3.66589400 & 8.05757100 \\
\hline $\mathrm{C}$ & 4.36084700 & 4.29880400 & 7.32669100 \\
\hline $\mathrm{C}$ & 5.53943800 & 3.47812300 & 7.44296200 \\
\hline $\mathrm{C}$ & 1.36292900 & -0.45908800 & 7.05078500 \\
\hline $\mathrm{C}$ & 1.05328100 & -1.19575000 & 5.84550300 \\
\hline $\mathrm{C}$ & 0.54294100 & -0.30377000 & 4.86530400 \\
\hline $\mathrm{C}$ & 0.62295500 & 1.01097000 & 5.42940900 \\
\hline $\mathrm{C}$ & 1.08087000 & 0.90257800 & 6.79261900 \\
\hline $\mathrm{C}$ & 2.59253900 & 2.98091500 & 1.64681500 \\
\hline $\mathrm{C}$ & 3.51876900 & 3.97985000 & 2.10512900 \\
\hline $\mathrm{C}$ & 2.89636400 & 4.70304800 & 3.17681100 \\
\hline $\mathrm{C}$ & 1.58569500 & 4.15910600 & 3.37782100 \\
\hline $\mathrm{C}$ & 1.41597000 & 3.08865100 & 2.44806500 \\
\hline $\mathrm{C}$ & 6.53265400 & 0.01101900 & 2.44109500 \\
\hline $\mathrm{C}$ & 5.42780400 & 0.07602200 & 1.51266200 \\
\hline $\mathrm{C}$ & 4.56984600 & -1.03248200 & 1.76527200 \\
\hline $\mathrm{C}$ & 5.13986600 & -1.80082800 & 2.84027800 \\
\hline $\mathrm{C}$ & 6.33909500 & -1.14361000 & 3.25519100 \\
\hline $\mathrm{H}$ & 5.86792000 & 1.54271600 & 8.53667000 \\
\hline $\mathrm{H}$ & 3.29656900 & 1.76398200 & 9.26213200 \\
\hline $\mathrm{H}$ & 2.30370400 & 4.04483300 & 8.17878400 \\
\hline $\mathrm{H}$ & 4.29036500 & 5.23482700 & 6.78763200 \\
\hline $\mathrm{H}$ & 6.51232100 & 3.69362900 & 7.02059000 \\
\hline $\mathrm{H}$ & 1.75301700 & -0.88291500 & 7.96652400 \\
\hline $\mathrm{H}$ & 1.21617100 & -2.25795800 & 5.71051200 \\
\hline $\mathrm{H}$ & 0.20973100 & -0.56223200 & 3.86941300 \\
\hline $\mathrm{H}$ & 0.32222100 & 1.92818000 & 4.94517300 \\
\hline $\mathrm{H}$ & 1.18870800 & 1.72630600 & 7.48225900 \\
\hline $\mathrm{H}$ & 2.75483300 & 2.27143400 & 0.84681600 \\
\hline $\mathrm{H}$ & 4.50347500 & 4.17369100 & 1.69956900 \\
\hline $\mathrm{H}$ & 3.33218700 & 5.53278400 & 3.71848600 \\
\hline $\mathrm{H}$ & 0.85625500 & 4.50285200 & 4.10038100 \\
\hline $\mathrm{H}$ & 0.54070100 & 2.45565100 & 2.36338700 \\
\hline $\mathrm{H}$ & 7.36772600 & 0.69735300 & 2.49486400 \\
\hline $\mathrm{H}$ & 5.28963900 & 0.82505400 & 0.74298100 \\
\hline $\mathrm{H}$ & 3.66423300 & -1.27493900 & 1.22361900 \\
\hline $\mathrm{H}$ & 4.77314000 & -2.75733600 & 3.19734100 \\
\hline $\mathrm{H}$ & 6.98927500 & -1.46196500 & 4.06176900 \\
\hline $\mathrm{H}$ & 2.91842200 & 0.61558600 & 3.59932300 \\
\hline $\mathrm{H}$ & 4.76148900 & 1.99004100 & 3.39424000 \\
\hline $\mathrm{H}$ & 4.28586600 & -1.98787400 & 5.04405600 \\
\hline $\mathrm{H}$ & 4.58342000 & -1.06969300 & 6.19226900 \\
\hline $\mathrm{H}$ & 2.71688300 & 2.95643300 & 5.43153800 \\
\hline $\mathrm{H}$ & 4.48548600 & 3.01996000 & 4.87836700 \\
\hline
\end{tabular}


$4 \mathrm{~b}+\mathrm{H}_{2} \mathrm{O}$

$\mathrm{E}(\mathrm{RB} 3 \mathrm{PW} 91)=-1308.22660606$ A.U.

Thermal correction to Gibbs Free Energy= 0.340716 A.U.

\begin{tabular}{|c|c|c|c|}
\hline $\mathrm{Ru}$ & 4.22919900 & 2.18873000 & 6.05510100 \\
\hline $\mathrm{Ru}$ & 2.14588300 & 0.50177700 & 5.20093000 \\
\hline $\mathrm{Ru}$ & 2.86972100 & 2.75106700 & 3.66284400 \\
\hline $\mathrm{Ru}$ & 4.59130500 & 0.53602000 & 3.80667500 \\
\hline $\mathrm{O}$ & 4.05596500 & 0.24181200 & 5.69381600 \\
\hline $\mathrm{O}$ & 5.53718300 & -1.43151200 & 7.17738500 \\
\hline $\mathrm{C}$ & 5.42486700 & 1.92609400 & 7.89592600 \\
\hline $\mathrm{C}$ & 4.15058900 & 2.49457500 & 8.20993300 \\
\hline $\mathrm{C}$ & 4.05802700 & 3.76844700 & 7.54505100 \\
\hline $\mathrm{C}$ & 5.27514800 & 3.96502800 & 6.82021800 \\
\hline $\mathrm{C}$ & 6.11657300 & 2.82448600 & 7.03107700 \\
\hline $\mathrm{C}$ & 1.11310100 & -0.93307400 & 6.50833800 \\
\hline $\mathrm{C}$ & 1.06162900 & -1.38829400 & 5.15242700 \\
\hline $\mathrm{C}$ & 0.34184200 & -0.39970800 & 4.39109100 \\
\hline $\mathrm{C}$ & -0.05282800 & 0.64643600 & 5.27655400 \\
\hline $\mathrm{C}$ & 0.44323000 & 0.32459900 & 6.58346100 \\
\hline $\mathrm{C}$ & 1.89464000 & 3.13565400 & 1.74679900 \\
\hline $\mathrm{C}$ & 3.00432700 & 4.03214900 & 1.88597900 \\
\hline $\mathrm{C}$ & 2.77133700 & 4.85277400 & 3.03231900 \\
\hline $\mathrm{C}$ & 1.51598600 & 4.47157000 & 3.60776400 \\
\hline $\mathrm{C}$ & 0.97528500 & 3.40939500 & 2.81184800 \\
\hline $\mathrm{C}$ & 6.71338700 & 0.09752600 & 3.33810600 \\
\hline $\mathrm{C}$ & 6.03697400 & 0.53075200 & 2.15117400 \\
\hline $\mathrm{C}$ & 4.97869200 & -0.39046100 & 1.87314400 \\
\hline $\mathrm{C}$ & 5.00366000 & -1.40093600 & 2.89855900 \\
\hline $\mathrm{C}$ & 6.08075600 & -1.09835700 & 3.78885900 \\
\hline $\mathrm{H}$ & 5.77170100 & 0.94202000 & 8.19023800 \\
\hline $\mathrm{H}$ & 3.39550800 & 2.05343200 & 8.84794300 \\
\hline $\mathrm{H}$ & 3.22809800 & 4.46085400 & 7.60139500 \\
\hline $\mathrm{H}$ & 5.52275000 & 4.82827600 & 6.21563000 \\
\hline $\mathrm{H}$ & 7.10016900 & 2.67069800 & 6.60662000 \\
\hline $\mathrm{H}$ & 1.59887200 & -1.44002000 & 7.33325400 \\
\hline $\mathrm{H}$ & 1.46414100 & -2.31851800 & 4.77228600 \\
\hline $\mathrm{H}$ & 0.12449100 & -0.45225800 & 3.33167900 \\
\hline $\mathrm{H}$ & -0.61684500 & 1.53144800 & 5.01381500 \\
\hline $\mathrm{H}$ & 0.32340600 & 0.92943800 & 7.47356400 \\
\hline $\mathrm{H}$ & 1.76868900 & 2.39459300 & 0.96792100 \\
\hline $\mathrm{H}$ & 3.86739000 & 4.08319300 & 1.23467900 \\
\hline $\mathrm{H}$ & 3.42857100 & 5.63087800 & 3.39875500 \\
\hline $\mathrm{H}$ & 1.05666600 & 4.91329500 & 4.48272500 \\
\hline $\mathrm{H}$ & 0.03170400 & 2.90713600 & 2.97752200 \\
\hline $\mathrm{H}$ & 7.55247100 & 0.59162400 & 3.81040900 \\
\hline $\mathrm{H}$ & 6.28891900 & 1.40558300 & 1.56538700 \\
\hline $\mathrm{H}$ & 4.29882500 & -0.34989800 & 1.03205900 \\
\hline $\mathrm{H}$ & 4.33607100 & -2.25003300 & 2.97072000 \\
\hline $\mathrm{H}$ & 6.32120100 & -1.62989500 & 4.70261600 \\
\hline $\mathrm{H}$ & 2.74128700 & 0.90180400 & 3.53084600 \\
\hline $\mathrm{H}$ & 4.39234900 & 2.11619100 & 3.12893900 \\
\hline $\mathrm{H}$ & 4.94851400 & -2.11456000 & 7.50383600 \\
\hline $\mathrm{H}$ & 4.95550300 & -0.84145000 & 6.64565400 \\
\hline $\mathrm{H}$ & 2.44709100 & 2.29270500 & 5.41498500 \\
\hline $\mathrm{H}$ & 4.14160500 & 3.28756400 & 4.71970000 \\
\hline
\end{tabular}

$\mathbf{T S}_{\mathrm{B} 1 / \mathrm{B5}}$

$\mathrm{E}(\mathrm{RB} 3 \mathrm{PW} 91)=-1308.00189567$ A.U.

Thermal correction to Gibbs Free Energy= 0.334046 A.U.

\begin{tabular}{|c|c|c|c|}
\hline $\mathrm{Ru}$ & 4.11328400 & 2.33142000 & 6.28934500 \\
\hline $\mathrm{Ru}$ & 2.52079100 & 0.08063900 & 5.58923500 \\
\hline $\mathrm{Ru}$ & 3.29455600 & 2.61723500 & 3.62951100 \\
\hline $\mathrm{Ru}$ & 4.75325000 & 0.27526800 & 3.62693700 \\
\hline $\mathrm{O}$ & 5.24899500 & 0.88256800 & 5.45292100 \\
\hline $\mathrm{O}$ & 4.01951100 & -0.92237700 & 6.05856400 \\
\hline $\mathrm{C}$ & 5.45598400 & 2.67080100 & 8.03296600 \\
\hline $\mathrm{C}$ & 4.12750100 & 2.50192500 & 8.52063800 \\
\hline $\mathrm{C}$ & 3.30983200 & 3.56219400 & 7.99743600 \\
\hline $\mathrm{C}$ & 4.12725400 & 4.35666100 & 7.15517400 \\
\hline $\mathrm{C}$ & 5.46788000 & 3.80609600 & 7.17349800 \\
\hline $\mathrm{C}$ & 1.11405100 & -1.49358500 & 6.31973000 \\
\hline $\mathrm{C}$ & 0.77745600 & -1.13968600 & 4.97284100 \\
\hline $\mathrm{C}$ & 0.33167500 & 0.22588700 & 4.96605100 \\
\hline $\mathrm{C}$ & 0.47361000 & 0.71944000 & 6.28809900 \\
\hline $\mathrm{C}$ & 0.94363000 & -0.34679500 & 7.13647700 \\
\hline $\mathrm{C}$ & 2.04520000 & 2.93349300 & 1.82653500 \\
\hline $\mathrm{C}$ & 3.27686300 & 3.65016700 & 1.70827100 \\
\hline $\mathrm{C}$ & 3.34611600 & 4.59588700 & 2.78551300 \\
\hline $\mathrm{C}$ & 2.14259300 & 4.47853300 & 3.56062300 \\
\hline $\mathrm{C}$ & 1.35737700 & 3.43836100 & 2.97758200 \\
\hline $\mathrm{C}$ & 6.75652600 & -0.21639000 & 2.80341800 \\
\hline $\mathrm{C}$ & 5.77873700 & -0.21832100 & 1.74791600 \\
\hline $\mathrm{C}$ & 4.85186500 & -1.27086000 & 2.01057200 \\
\hline $\mathrm{C}$ & 5.25965500 & -1.92018300 & 3.22469400 \\
\hline $\mathrm{C}$ & 6.42510500 & -1.27729600 & 3.70094400 \\
\hline $\mathrm{H}$ & 6.28845700 & 2.00538800 & 8.22140800 \\
\hline $\mathrm{H}$ & 3.80202900 & 1.72195000 & 9.19733400 \\
\hline $\mathrm{H}$ & 2.25848300 & 3.71730700 & 8.20314900 \\
\hline $\mathrm{H}$ & 3.81390100 & 5.23981600 & 6.61335900 \\
\hline $\mathrm{H}$ & 6.33083000 & 4.20334300 & 6.65505400 \\
\hline $\mathrm{H}$ & 1.50396100 & -2.45006200 & 6.64321000 \\
\hline $\mathrm{H}$ & 0.79558800 & -1.80573800 & 4.11932600 \\
\hline $\mathrm{H}$ & -0.02919200 & 0.77106600 & 4.10499400 \\
\hline $\mathrm{H}$ & 0.25915000 & 1.72950100 & 6.61377200 \\
\hline $\mathrm{H}$ & 1.12192100 & -0.28873000 & 8.20227200 \\
\hline $\mathrm{H}$ & 1.69243300 & 2.15491700 & 1.16233400 \\
\hline $\mathrm{H}$ & 4.02896900 & 3.50509600 & 0.94331200 \\
\hline $\mathrm{H}$ & 4.15259200 & 5.29699800 & 2.95924600 \\
\hline $\mathrm{H}$ & 1.87914400 & 5.06502000 & 4.43085800 \\
\hline $\mathrm{H}$ & 0.39610500 & 3.09768100 & 3.34031400 \\
\hline $\mathrm{H}$ & 7.60025100 & 0.45553300 & 2.89257700 \\
\hline $\mathrm{H}$ & 5.76839100 & 0.44356000 & 0.89109300 \\
\hline $\mathrm{H}$ & 4.00852400 & -1.54804100 & 1.39112900 \\
\hline $\mathrm{H}$ & 4.73580000 & -2.72164700 & 3.72810500 \\
\hline $\mathrm{H}$ & 6.91121600 & -1.48330900 & 4.64537200 \\
\hline $\mathrm{H}$ & 3.51517900 & 0.97731500 & 2.79191500 \\
\hline $\mathrm{H}$ & 4.93860300 & 1.94612300 & 3.34730100 \\
\hline $\mathrm{H}$ & 3.18002800 & -0.28596900 & 4.01837200 \\
\hline $\mathrm{H}$ & 3.35414400 & 0.95425900 & 6.89166000 \\
\hline $\mathrm{H}$ & 2.68735600 & 2.09026700 & 5.27037700 \\
\hline $\mathrm{H}$ & 4.51246500 & 3.08792800 & 4.78522700 \\
\hline
\end{tabular}


B5

$\mathrm{E}($ RB3PW91 $)=-1308.05347832$ A.U.

Thermal correction to Gibbs Free Energy= 0.331918 A.U.

\begin{tabular}{|c|c|c|c|}
\hline $\mathrm{Ru}$ & 4.28017600 & 2.25413000 & 6.21328600 \\
\hline $\mathrm{Ru}$ & 2.76954700 & -0.48663500 & 5.95523200 \\
\hline $\mathrm{Ru}$ & 3.55096700 & 3.14802200 & 3.48019000 \\
\hline $\mathrm{Ru}$ & 4.86442700 & 0.51768500 & 3.85942800 \\
\hline $\mathrm{O}$ & 5.70638500 & 1.54241500 & 5.20296700 \\
\hline $\mathrm{O}$ & 4.21054600 & -1.10966600 & 6.75733400 \\
\hline $\mathrm{C}$ & 4.54921100 & 1.86224100 & 8.36048200 \\
\hline $\mathrm{C}$ & 3.25662300 & 2.48714000 & 8.21732300 \\
\hline $\mathrm{C}$ & 3.43918600 & 3.76847100 & 7.65369800 \\
\hline $\mathrm{C}$ & 4.84844100 & 3.96584100 & 7.44332300 \\
\hline $\mathrm{C}$ & 5.53048400 & 2.79912000 & 7.92203900 \\
\hline $\mathrm{C}$ & 1.18793700 & -1.81397000 & 6.55265000 \\
\hline $\mathrm{C}$ & 1.25522700 & -1.82936000 & 5.11850800 \\
\hline $\mathrm{C}$ & 0.83315400 & -0.54131400 & 4.63738800 \\
\hline $\mathrm{C}$ & 0.56468300 & 0.27729600 & 5.74489000 \\
\hline $\mathrm{C}$ & 0.81393000 & -0.48300500 & 6.94024000 \\
\hline $\mathrm{C}$ & 1.70132800 & 3.24844600 & 2.09020900 \\
\hline $\mathrm{C}$ & 2.89169300 & 3.70633000 & 1.43979900 \\
\hline $\mathrm{C}$ & 3.37012400 & 4.85139300 & 2.16268800 \\
\hline $\mathrm{C}$ & 2.47967700 & 5.07741300 & 3.26658800 \\
\hline $\mathrm{C}$ & 1.45037900 & 4.08516800 & 3.20429800 \\
\hline $\mathrm{C}$ & 6.18285700 & 0.08328100 & 2.17376700 \\
\hline $\mathrm{C}$ & 4.90343500 & -0.48704300 & 1.84521800 \\
\hline $\mathrm{C}$ & 4.60170500 & -1.47033600 & 2.81269000 \\
\hline $\mathrm{C}$ & 5.68410900 & -1.52023900 & 3.76584100 \\
\hline $\mathrm{C}$ & 6.67650800 & -0.59013700 & 3.33868700 \\
\hline $\mathrm{H}$ & 4.73330500 & 0.86320300 & 8.73383200 \\
\hline $\mathrm{H}$ & 2.30860900 & 2.03387500 & 8.47367000 \\
\hline $\mathrm{H}$ & 2.65818800 & 4.47018700 & 7.39055800 \\
\hline $\mathrm{H}$ & 5.30855000 & 4.85391300 & 7.02845700 \\
\hline $\mathrm{H}$ & 6.59858500 & 2.62700800 & 7.88179700 \\
\hline $\mathrm{H}$ & 1.39244500 & -2.64515900 & 7.21641300 \\
\hline $\mathrm{H}$ & 1.49639400 & -2.68665900 & 4.50223500 \\
\hline $\mathrm{H}$ & 0.80090800 & -0.23501600 & 3.59981800 \\
\hline $\mathrm{H}$ & 0.28852600 & 1.32309200 & 5.70845300 \\
\hline $\mathrm{H}$ & 0.66110100 & -0.13529800 & 7.95440600 \\
\hline $\mathrm{H}$ & 1.10117800 & 2.39929000 & 1.78782600 \\
\hline $\mathrm{H}$ & 3.31840700 & 3.29942300 & 0.53234200 \\
\hline $\mathrm{H}$ & 4.22150000 & 5.46397100 & 1.89550800 \\
\hline $\mathrm{H}$ & 2.54184800 & 5.88604600 & 3.98307000 \\
\hline $\mathrm{H}$ & 0.62749200 & 3.98201200 & 3.90071600 \\
\hline $\mathrm{H}$ & 6.69086200 & 0.86108500 & 1.61747400 \\
\hline $\mathrm{H}$ & 4.27224100 & -0.18559600 & 1.01920600 \\
\hline $\mathrm{H}$ & 3.69941100 & -2.06529700 & 2.85610700 \\
\hline $\mathrm{H}$ & 5.72957700 & -2.15195300 & 4.64347600 \\
\hline $\mathrm{H}$ & 7.60440300 & -0.37244700 & 3.85208500 \\
\hline $\mathrm{H}$ & 3.52804800 & 1.47350800 & 3.19237100 \\
\hline $\mathrm{H}$ & 5.04877500 & 2.82489700 & 3.06783900 \\
\hline $\mathrm{H}$ & 3.29880600 & -0.09347100 & 4.35829600 \\
\hline $\mathrm{H}$ & 2.85667600 & 1.22363400 & 6.14606900 \\
\hline $\mathrm{H}$ & 3.07323100 & 2.87743700 & 5.09029800 \\
\hline $\mathrm{H}$ & 4.75382100 & 3.75628200 & 4.31747700 \\
\hline
\end{tabular}

1b

$\mathrm{E}(\mathrm{RB} 3 \mathrm{PW} 91)=-1549.29186336$ A.U.

Thermal correction to Enthalpy=0.577936 A.U.

\begin{tabular}{|c|c|c|c|}
\hline $\mathrm{Ru}$ & 4.33516300 & & 5.76768800 \\
\hline $\mathrm{Ru}$ & 2.47928700 & 0.38008600 & 5.57710800 \\
\hline $\mathrm{Ru}$ & 2.68010400 & 2.25308000 & 3.58908600 \\
\hline $\mathrm{Ru}$ & 4.64188200 & 0.35538100 & 3.89831900 \\
\hline $\mathrm{C}$ & 5.76631900 & 2.67055100 & 7.41010600 \\
\hline $\mathrm{C}$ & 4.44835600 & 3.07248600 & 7.83423300 \\
\hline $\mathrm{C}$ & 4.03762300 & 4.17047700 & 6.99086200 \\
\hline $\mathrm{C}$ & 5.07831500 & 4.42383700 & 6.03872700 \\
\hline $\mathrm{C}$ & 6.15687700 & 3.49136100 & 6.30265900 \\
\hline $\mathrm{C}$ & 1.38079700 & -1.50829700 & 5.62985700 \\
\hline $\mathrm{C}$ & 0.44855900 & -0.41196600 & 5.61168900 \\
\hline $\mathrm{C}$ & 0.66773300 & 0.35992800 & 6.79322300 \\
\hline $\mathrm{C}$ & 1.73473800 & -0.23731100 & 7.53691400 \\
\hline $\mathrm{C}$ & 2.16935900 & -1.39901600 & 6.81068700 \\
\hline $\mathrm{C}$ & 1.28033500 & 2.19382700 & 1.91532600 \\
\hline $\mathrm{C}$ & 2.46142400 & 2.89234100 & 1.51073200 \\
\hline $\mathrm{C}$ & 2.58743000 & 4.05181900 & 2.35152900 \\
\hline $\mathrm{C}$ & 1.49547800 & 4.05904400 & 3.26597200 \\
\hline $\mathrm{C}$ & 0.68180400 & 2.90194000 & 3.00274800 \\
\hline $\mathrm{C}$ & 5.52394000 & -1.63467800 & 3.81609200 \\
\hline $\mathrm{C}$ & 6.58963600 & -0.65393200 & 3.87937300 \\
\hline $\mathrm{C}$ & 6.48747600 & 0.18229700 & 2.72153200 \\
\hline $\mathrm{C}$ & 5.36035000 & -0.26049000 & 1.93676500 \\
\hline $\mathrm{C}$ & 4.78168200 & -1.39613600 & 2.61295800 \\
\hline $\mathrm{C}$ & 5.11776600 & 5.53681500 & 5.03877000 \\
\hline $\mathrm{C}$ & 2.77007400 & 4.95118200 & 7.14646800 \\
\hline $\mathrm{C}$ & 3.72438500 & 2.58453200 & 9.04965400 \\
\hline $\mathrm{C}$ & 6.60507300 & 1.62511100 & 8.07624300 \\
\hline $\mathrm{C}$ & 7.49767900 & 3.51251700 & 5.63923800 \\
\hline $\mathrm{C}$ & 7.68871400 & -0.64516500 & 4.89481800 \\
\hline $\mathrm{C}$ & 5.33888300 & -2.77835600 & 4.76416700 \\
\hline $\mathrm{C}$ & 3.64427600 & -2.22179200 & 2.09808200 \\
\hline $\mathrm{C}$ & 4.98620800 & 0.23718500 & 0.57584300 \\
\hline $\mathrm{C}$ & 7.42859200 & 1.27970100 & 2.33442900 \\
\hline $\mathrm{H}$ & 2.78768100 & 0.40690000 & 3.75062100 \\
\hline $\mathrm{H}$ & 4.51484200 & 2.22884300 & 3.89641900 \\
\hline $\mathrm{H}$ & 4.33788100 & 0.51886700 & 5.74579700 \\
\hline $\mathrm{H}$ & 2.50949500 & 2.22329900 & 5.43362100 \\
\hline $\mathrm{H}$ & 1.46640000 & -2.27777400 & 4.87342800 \\
\hline $\mathrm{H}$ & -0.29562000 & -0.21772700 & 4.85025800 \\
\hline $\mathrm{H}$ & 0.13368200 & 1.26214400 & 7.06576300 \\
\hline $\mathrm{H}$ & 2.11956600 & 0.10251100 & 8.48853400 \\
\hline $\mathrm{H}$ & 2.96678100 & -2.06910700 & 7.10600600 \\
\hline $\mathrm{H}$ & 0.91444500 & 1.26859500 & 1.48682500 \\
\hline $\mathrm{H}$ & 3.12262600 & 2.61682200 & 0.70084800 \\
\hline $\mathrm{H}$ & 3.38018100 & 4.78750300 & 2.30354700 \\
\hline $\mathrm{H}$ & 1.30955800 & 4.80197800 & 4.03105900 \\
\hline $\mathrm{H}$ & -0.22863600 & 2.62878300 & 3.52017600 \\
\hline $\mathrm{H}$ & 4.10998300 & 5.84087900 & 4.74166500 \\
\hline $\mathrm{H}$ & 5.62587600 & 6.42085900 & 5.45134100 \\
\hline $\mathrm{H}$ & 5.65284800 & 5.23859400 & 4.13183300 \\
\hline $\mathrm{H}$ & 2.89780500 & 5.75692800 & 7.88380700 \\
\hline $\mathrm{H}$ & 2.46376200 & 5.40912000 & 6.20204000 \\
\hline $\mathrm{H}$ & 1.94803600 & 4.31417100 & 7.48640800 \\
\hline
\end{tabular}




$\begin{array}{lrrr}\mathrm{H} & 3.97226300 & 3.19622000 & 9.92997700 \\ \mathrm{H} & 2.63976400 & 2.62578800 & 8.91145600 \\ \mathrm{H} & 3.98908200 & 1.54903400 & 9.28322600 \\ \mathrm{H} & 7.15318700 & 2.05267500 & 8.92840700 \\ \mathrm{H} & 5.99041000 & 0.80269100 & 8.45509700 \\ \mathrm{H} & 7.33996300 & 1.19931100 & 7.38808800 \\ \mathrm{H} & 8.16441700 & 4.23917200 & 6.12680800 \\ \mathrm{H} & 7.98454900 & 2.53470400 & 5.68811400 \\ \mathrm{H} & 7.41828700 & 3.79264500 & 4.58464900 \\ \mathrm{H} & 7.31680900 & -0.90184100 & 5.89140100 \\ \mathrm{H} & 8.46700500 & -1.37704800 & 4.63246700 \\ \mathrm{H} & 8.16966300 & 0.33475100 & 4.96020500 \\ \mathrm{H} & 5.96972400 & -3.63331900 & 4.47922700 \\ \mathrm{H} & 5.60542400 & -2.49536700 & 5.78722200 \\ \mathrm{H} & 4.30050300 & -3.12172400 & 4.77772300 \\ \mathrm{H} & 4.00844500 & -2.99713000 & 1.40862400 \\ \mathrm{H} & 3.11365300 & -2.72246300 & 2.91263800 \\ \mathrm{H} & 2.91809100 & -1.60764200 & 1.55707400 \\ \mathrm{H} & 5.52296200 & -0.31576900 & -0.20950700 \\ \mathrm{H} & 3.91426900 & 0.12112800 & 0.38986700 \\ \mathrm{H} & 5.23039600 & 1.29730600 & 0.45841400 \\ \mathrm{H} & 8.20804200 & 0.90272700 & 1.65643500 \\ \mathrm{H} & 6.90367400 & 2.09088600 & 1.82061200 \\ \mathrm{H} & 7.92517600 & 1.70931600 & 3.20804500\end{array}$

\section{3a}

$\mathrm{E}(\mathrm{RB} 3 \mathrm{PW} 91)=-1698.68232141 \mathrm{~A} . \mathrm{U}$.

Thermal correction to Enthalpy= 0.003777 A.U. Entropy $($ Total $)=536.266 \mathrm{Cal} / \mathrm{Mol}-$ Kelvin

$\begin{array}{lrrr}\mathrm{Ru} & 4.50622300 & 2.42452500 & 5.86103500 \\ \mathrm{Ru} & 2.18910300 & 0.33432800 & 5.52176100 \\ \mathrm{Ru} & 2.69151400 & 2.39817100 & 3.83459000 \\ \mathrm{Ru} & 4.51076500 & 0.36141000 & 4.09993400 \\ \mathrm{O} & 4.15151500 & 0.43847400 & 6.03710500 \\ \mathrm{O} & 2.48469100 & 2.31326500 & 5.79952700 \\ \mathrm{C} & 6.29917800 & 2.57647800 & 7.09682500 \\ \mathrm{C} & 5.17191800 & 3.11507000 & 7.81232700 \\ \mathrm{C} & 4.71043200 & 4.25825700 & 7.07798400 \\ \mathrm{C} & 5.54924000 & 4.43511300 & 5.94259400 \\ \mathrm{C} & 6.52807200 & 3.39381000 & 5.95038700 \\ \mathrm{C} & 1.24494800 & -1.72458100 & 5.40495000 \\ \mathrm{C} & 0.31064300 & -0.72721300 & 4.98249700 \\ \mathrm{C} & 0.09069900 & 0.17117400 & 6.07233800 \\ \mathrm{C} & 0.89970300 & -0.27234400 & 7.17869200 \\ \mathrm{C} & 1.61322000 & -1.43475100 & 6.74742000 \\ \mathrm{C} & 1.80019200 & 2.53178400 & 1.83998100 \\ \mathrm{C} & 2.88742300 & 3.46008600 & 1.91724800 \\ \mathrm{C} & 2.56308100 & 4.43605600 & 2.91777500 \\ \mathrm{C} & 1.28302500 & 4.11113400 & 3.44776700 \\ \mathrm{C} & 0.80549700 & 2.93840300 & 2.79451000 \\ \mathrm{C} & 5.76140400 & -1.49094800 & 4.07039400 \\ \mathrm{C} & 6.57228900 & -0.34028200 & 3.82160900 \\ \mathrm{C} & 6.10322500 & 0.26732700 & 2.60435900 \\ \mathrm{C} & 5.01266300 & -0.51706800 & 2.11418900 \\ \mathrm{C} & 4.80039800 & -1.59750000 & 3.02588900 \\ \mathrm{C} & 0.96853300 & 0.37220300 & 8.57562500 \\ \mathrm{C} & -0.86279100 & 1.38048800 & 6.07630600\end{array}$

\begin{tabular}{|c|c|c|c|}
\hline C & -0.34755700 & -0.64284300 & 3.59280100 \\
\hline $\mathrm{C}$ & 1.78539500 & -2.88459400 & 4.54827700 \\
\hline C & 2.65163800 & -2.20924000 & 7.58015900 \\
\hline $\mathrm{C}$ & 5.86384200 & -2.41659600 & 5.29688500 \\
\hline $\mathrm{C}$ & 3.71128600 & -2.67955500 & 2.90508800 \\
\hline $\mathrm{C}$ & 4.19944500 & -0.24472900 & 0.83508400 \\
\hline $\mathrm{C}$ & 6.70342700 & 1.51093900 & 1.92260700 \\
\hline C & 7.75702000 & 0.13958600 & 4.68052600 \\
\hline $\mathrm{H}$ & 6.87525700 & 1.70547700 & 7.38339100 \\
\hline $\mathrm{H}$ & 4.76606200 & 2.74530000 & 8.74498600 \\
\hline $\mathrm{H}$ & 3.85432700 & 4.87077000 & 7.33340200 \\
\hline $\mathrm{H}$ & 5.44906300 & 5.20685100 & 5.19032600 \\
\hline $\mathrm{H}$ & 7.30214400 & 3.24582300 & 5.20771800 \\
\hline $\mathrm{H}$ & 1.72436500 & 1.69064300 & 1.16285500 \\
\hline $\mathrm{H}$ & 3.78534100 & 3.44486500 & 1.31278000 \\
\hline $\mathrm{H}$ & 3.18129100 & 5.27306300 & 3.21507800 \\
\hline $\mathrm{H}$ & 0.78592000 & 4.62912000 & 4.25864500 \\
\hline $\mathrm{H}$ & -0.14179900 & 2.44831000 & 2.97782500 \\
\hline $\mathrm{H}$ & 2.71077000 & 0.57834400 & 3.77422900 \\
\hline $\mathrm{H}$ & 4.49227100 & 2.18498400 & 4.02708900 \\
\hline $\mathrm{H}$ & 0.29752600 & -0.13587700 & 9.23631000 \\
\hline $\mathrm{H}$ & 1.96655500 & 0.29746500 & 8.95412900 \\
\hline $\mathrm{H}$ & 0.68934000 & 1.40285700 & 8.50703100 \\
\hline $\mathrm{H}$ & -1.59565400 & 1.25433100 & 6.84565500 \\
\hline $\mathrm{H}$ & -0.30411800 & 2.27457400 & 6.25904800 \\
\hline $\mathrm{H}$ & -1.35109200 & 1.45279700 & 5.12697300 \\
\hline $\mathrm{H}$ & -1.40937700 & -0.72594200 & 3.69542300 \\
\hline $\mathrm{H}$ & -0.10537900 & 0.29581500 & 3.13984300 \\
\hline $\mathrm{H}$ & 0.01476600 & -1.43978300 & 2.97756900 \\
\hline $\mathrm{H}$ & 0.97147500 & -3.50126600 & 4.22867000 \\
\hline $\mathrm{H}$ & 2.29151500 & -2.49000400 & 3.69209900 \\
\hline $\mathrm{H}$ & 2.46869900 & -3.46849400 & 5.12884200 \\
\hline $\mathrm{H}$ & 2.48474700 & -3.26041100 & 7.47021900 \\
\hline $\mathrm{H}$ & 3.63597300 & -1.96666700 & 7.23789700 \\
\hline $\mathrm{H}$ & 2.55569200 & -1.93876000 & 8.61095300 \\
\hline $\mathrm{H}$ & 5.23257300 & -2.04586400 & 6.07724200 \\
\hline $\mathrm{H}$ & 5.55315300 & -3.40389000 & 5.02554700 \\
\hline $\mathrm{H}$ & 6.87697500 & -2.44318100 & 5.64003900 \\
\hline $\mathrm{H}$ & 4.17177900 & -3.64286200 & 2.83519200 \\
\hline $\mathrm{H}$ & 3.07977000 & -2.64790000 & 3.76827100 \\
\hline $\mathrm{H}$ & 3.12558900 & -2.49971800 & 2.02786600 \\
\hline $\mathrm{H}$ & 4.27488200 & -1.08624900 & 0.17853800 \\
\hline $\mathrm{H}$ & 3.17334900 & -0.08386900 & 1.09228100 \\
\hline $\mathrm{H}$ & 4.58507800 & 0.62515400 & 0.34570600 \\
\hline $\mathrm{H}$ & 7.06222500 & 1.24622200 & 0.94993600 \\
\hline $\mathrm{H}$ & 5.95081200 & 2.26607500 & 1.83182500 \\
\hline $\mathrm{H}$ & 7.51426600 & 1.88458900 & 2.51237400 \\
\hline $\mathrm{H}$ & 8.67282000 & -0.21213900 & 4.25332600 \\
\hline $\mathrm{H}$ & 7.76530400 & 1.20911800 & 4.71105000 \\
\hline $\mathrm{H}$ & 7.65609300 & -0.24480800 & 5.67398300 \\
\hline
\end{tabular}

3b

$\mathrm{E}(\mathrm{RB} 3 \mathrm{PW} 91)=-1698.68716231$ A.U.

Thermal correction to Enthalpy $=0.003777$ A.U.

Entropy $($ Total $)=536.250 \mathrm{Cal} / \mathrm{Mol}-\mathrm{Kelvin}$

$\begin{array}{llll}\mathrm{Ru} & 4.50622300 & 2.42452500 & 5.86103500 \\ \mathrm{Ru} & 2.18910300 & 0.33432800 & 5.52176100\end{array}$




\begin{tabular}{|c|c|c|c|}
\hline $\mathrm{Ru}$ & 2.69151400 & 2.39817100 & 3.83459000 \\
\hline $\mathrm{Ru}$ & 4.51076500 & 0.36141000 & 4.09993400 \\
\hline $\mathrm{O}$ & 4.15151500 & 0.43847400 & 6.03710500 \\
\hline $\mathrm{O}$ & 2.48469100 & 2.31326500 & 5.79952700 \\
\hline $\mathrm{C}$ & 6.29917800 & 2.57647800 & 7.09682500 \\
\hline $\mathrm{C}$ & 5.17191800 & 3.11507000 & 7.81232700 \\
\hline $\mathrm{C}$ & 4.71043200 & 4.25825700 & 7.07798400 \\
\hline $\mathrm{C}$ & 5.54924000 & 4.43511300 & 5.94259400 \\
\hline $\mathrm{C}$ & 6.52807200 & 3.39381000 & 5.95038700 \\
\hline $\mathrm{C}$ & 1.24494800 & -1.72458100 & 5.40495000 \\
\hline $\mathrm{C}$ & 0.31064300 & -0.72721300 & 4.98249700 \\
\hline $\mathrm{C}$ & 0.09069900 & 0.17117400 & 6.07233800 \\
\hline $\mathrm{C}$ & 0.89970300 & -0.27234400 & 7.17869200 \\
\hline $\mathrm{C}$ & 1.61322000 & -1.43475100 & 6.74742000 \\
\hline $\mathrm{C}$ & 1.80019200 & 2.53178400 & 1.83998100 \\
\hline $\mathrm{C}$ & 2.88742300 & 3.46008600 & 1.91724800 \\
\hline $\mathrm{C}$ & 2.56308100 & 4.43605600 & 2.91777500 \\
\hline $\mathrm{C}$ & 1.28302500 & 4.11113400 & 3.44776700 \\
\hline $\mathrm{C}$ & 0.80549700 & 2.93840300 & 2.79451000 \\
\hline $\mathrm{C}$ & 5.76140400 & -1.49094800 & 4.07039400 \\
\hline $\mathrm{C}$ & 6.57228900 & -0.34028200 & 3.82160900 \\
\hline $\mathrm{C}$ & 6.10322500 & 0.26732700 & 2.60435900 \\
\hline $\mathrm{C}$ & 5.01266300 & -0.51706800 & 2.11418900 \\
\hline $\mathrm{C}$ & 4.80039800 & -1.59750000 & 3.02588900 \\
\hline $\mathrm{C}$ & 0.57617100 & 4.84768000 & 4.60078800 \\
\hline $\mathrm{C}$ & 3.44281200 & 5.62714100 & 3.34084600 \\
\hline $\mathrm{C}$ & -0.54252600 & 2.24099000 & 3.05537100 \\
\hline $\mathrm{C}$ & 1.69231600 & 1.33512900 & 0.87666300 \\
\hline $\mathrm{C}$ & 4.16479600 & 3.43843300 & 1.05733500 \\
\hline $\mathrm{C}$ & 6.70342700 & 1.51093900 & 1.92260700 \\
\hline $\mathrm{C}$ & 4.19944500 & -0.24472900 & 0.83508400 \\
\hline $\mathrm{C}$ & 3.71128600 & -2.67955500 & 2.90508800 \\
\hline $\mathrm{C}$ & 5.86384200 & -2.41659600 & 5.29688500 \\
\hline $\mathrm{C}$ & 7.75702000 & 0.13958600 & 4.68052600 \\
\hline $\mathrm{H}$ & 6.87525700 & 1.70547700 & 7.38339100 \\
\hline $\mathrm{H}$ & 4.76606200 & 2.74530000 & 8.74498600 \\
\hline $\mathrm{H}$ & 3.85432700 & 4.87077000 & 7.33340200 \\
\hline $\mathrm{H}$ & 5.44906300 & 5.20685100 & 5.19032600 \\
\hline $\mathrm{H}$ & 7.30214400 & 3.24582300 & 5.20771800 \\
\hline $\mathrm{H}$ & 1.62476800 & -2.53982500 & 4.80289000 \\
\hline $\mathrm{H}$ & -0.15212000 & -0.66789500 & 4.00543800 \\
\hline $\mathrm{H}$ & -0.57973100 & 1.02148200 & 6.07512800 \\
\hline $\mathrm{H}$ & 0.94808000 & 0.18067700 & 8.16052800 \\
\hline $\mathrm{H}$ & 2.34369100 & -1.97956200 & 7.33320700 \\
\hline $\mathrm{H}$ & 2.71077000 & 0.57834400 & 3.77422900 \\
\hline $\mathrm{H}$ & 4.49227100 & 2.18498400 & 4.02708900 \\
\hline $\mathrm{H}$ & -0.48350400 & 4.74726400 & 4.49167600 \\
\hline $\mathrm{H}$ & 0.88036200 & 4.42329300 & 5.53473900 \\
\hline $\mathrm{H}$ & 0.84052900 & 5.88423800 & 4.57707400 \\
\hline $\mathrm{H}$ & 2.91717900 & 6.54118200 & 3.15880100 \\
\hline $\mathrm{H}$ & 3.67184900 & 5.54891200 & 4.38311400 \\
\hline $\mathrm{H}$ & 4.35064900 & 5.61890300 & 2.77457400 \\
\hline $\mathrm{H}$ & -0.59214100 & 1.93204200 & 4.07859600 \\
\hline $\mathrm{H}$ & -1.34200500 & 2.92157500 & 2.84912600 \\
\hline $\mathrm{H}$ & -0.63004500 & 1.38478600 & 2.41963900 \\
\hline $\mathrm{H}$ & 1.58269300 & 0.43226000 & 1.44032200 \\
\hline $\mathrm{H}$ & 0.84123400 & 1.46442300 & 0.24117700 \\
\hline
\end{tabular}

$\begin{array}{rrrr}\mathrm{H} & 2.57806900 & 1.27726300 & 0.27917400 \\ \mathrm{H} & 4.02533900 & 4.05752900 & 0.19584100 \\ \mathrm{H} & 4.98815600 & 3.80764200 & 1.63236800 \\ \mathrm{H} & 4.36841700 & 2.43508300 & 0.74632600 \\ \mathrm{H} & 7.11956500 & 2.15898100 & 2.66541900 \\ \mathrm{H} & 7.47180600 & 1.20869200 & 1.24206200 \\ \mathrm{H} & 5.93593300 & 2.02921300 & 1.38665400 \\ \mathrm{H} & 3.71890700 & 0.70803000 & 0.91403800 \\ \mathrm{H} & 4.85462100 & -0.24473500 & -0.01087300 \\ \mathrm{H} & 3.45978000 & -1.00825900 & 0.71336100 \\ \mathrm{H} & 2.74658900 & -2.21669600 & 2.90971000 \\ \mathrm{H} & 3.84390500 & -3.21852700 & 1.99030800 \\ \mathrm{H} & 3.78664300 & -3.35525800 & 3.73131200 \\ \mathrm{H} & 4.94968800 & -2.37627900 & 5.85150000 \\ \mathrm{H} & 6.03999900 & -3.42078900 & 4.97213100 \\ \mathrm{H} & 6.67301400 & -2.09586600 & 5.91919800 \\ \mathrm{H} & 7.38690500 & 0.63277500 & 5.55498600 \\ \mathrm{H} & 8.35141600 & -0.70167200 & 4.97014600 \\ \mathrm{H} & 8.35589700 & 0.82106900 & 4.11322800\end{array}$

3c

$\mathrm{E}(\mathrm{RB} 3 \mathrm{PW} 91)=-1698.68868843$ A.U.

Thermal correction to Enthalpy=0 0.003777 A.U.

Entropy $($ Total $)=536.265 \mathrm{Cal} / \mathrm{Mol}-$ Kelvin

$\begin{array}{lrrr}\mathrm{Ru} & 4.50622300 & 2.42452500 & 5.86103500 \\ \mathrm{Ru} & 2.18910300 & 0.33432800 & 5.52176100 \\ \mathrm{Ru} & 2.69151400 & 2.39817100 & 3.83459000 \\ \mathrm{Ru} & 4.51076500 & 0.36141000 & 4.09993400 \\ \mathrm{O} & 4.15151500 & 0.43847400 & 6.03710500 \\ \mathrm{O} & 2.48469100 & 2.31326500 & 5.79952700 \\ \mathrm{C} & 6.29917800 & 2.57647800 & 7.09682500 \\ \mathrm{C} & 5.17191800 & 3.11507000 & 7.81232700 \\ \mathrm{C} & 4.71043200 & 4.25825700 & 7.07798400 \\ \mathrm{C} & 5.54924000 & 4.43511300 & 5.94259400 \\ \mathrm{C} & 6.52807200 & 3.39381000 & 5.95038700 \\ \mathrm{C} & 1.24494800 & -1.72458100 & 5.40495000 \\ \mathrm{C} & 0.31064300 & -0.72721300 & 4.98249700 \\ \mathrm{C} & 0.09069900 & 0.17117400 & 6.07233800 \\ \mathrm{C} & 0.89970300 & -0.27234400 & 7.17869200 \\ \mathrm{C} & 1.61322000 & -1.43475100 & 6.74742000 \\ \mathrm{C} & 1.80019200 & 2.53178400 & 1.83998100 \\ \mathrm{C} & 2.88742300 & 3.46008600 & 1.91724800 \\ \mathrm{C} & 2.56308100 & 4.43605600 & 2.91777500 \\ \mathrm{C} & 1.28302500 & 4.11113400 & 3.44776700 \\ \mathrm{C} & 0.80549700 & 2.93840300 & 2.79451000 \\ \mathrm{C} & 5.76140400 & -1.49094800 & 4.07039400 \\ \mathrm{C} & 6.57228900 & -0.34028200 & 3.82160900 \\ \mathrm{C} & 6.10322500 & 0.26732700 & 2.60435900 \\ \mathrm{C} & 5.01266300 & -0.51706800 & 2.11418900 \\ \mathrm{C} & 4.80039800 & -1.59750000 & 3.02588900 \\ \mathrm{C} & 0.96853300 & 0.37220300 & 8.57562500 \\ \mathrm{C} & -0.86279100 & 1.38048800 & 6.07630600 \\ \mathrm{C} & -0.34755700 & -0.64284300 & 3.59280100 \\ \mathrm{C} & 1.78539500 & -2.88459400 & 4.54827700 \\ \mathrm{C} & 2.65163800 & -2.20924000 & 7.58015900 \\ \mathrm{C} & 4.59441000 & 2.58891000 & 9.13944400 \\ \mathrm{C} & 3.49329800 & 5.12907300 & 7.44111500\end{array}$




\begin{tabular}{|c|c|c|c|}
\hline C & 5.40670800 & 5.53314600 & 4.87226300 \\
\hline C & 7.62889800 & 3.18335400 & 4.89422000 \\
\hline C & 7.11843900 & 1.33779900 & 7.50436000 \\
\hline $\mathrm{H}$ & 1.72436500 & 1.69064300 & 1.16285500 \\
\hline $\mathrm{H}$ & 3.78534100 & 3.44486500 & 1.31278000 \\
\hline $\mathrm{H}$ & 3.18129100 & 5.27306300 & 3.21507800 \\
\hline $\mathrm{H}$ & 0.78592000 & 4.62912000 & 4.25864500 \\
\hline $\mathrm{H}$ & -0.14179900 & 2.44831000 & 2.97782500 \\
\hline $\mathrm{H}$ & 5.83344100 & -2.14188600 & 4.93289200 \\
\hline $\mathrm{H}$ & 7.40473300 & -0.00310600 & 4.42512200 \\
\hline $\mathrm{H}$ & 6.52514400 & 1.14153900 & 2.12511300 \\
\hline $\mathrm{H}$ & 4.44108900 & -0.32565300 & 1.21516400 \\
\hline $\mathrm{H}$ & 4.03480900 & -2.35812800 & 2.94097200 \\
\hline $\mathrm{H}$ & 2.71077000 & 0.57834400 & 3.77422900 \\
\hline $\mathrm{H}$ & 4.49227100 & 2.18498400 & 4.02708900 \\
\hline $\mathrm{H}$ & 0.29752600 & -0.13587700 & 9.23631000 \\
\hline $\mathrm{H}$ & 1.96655500 & 0.29746500 & 8.95412900 \\
\hline $\mathrm{H}$ & 0.68934000 & 1.40285700 & 8.50703100 \\
\hline $\mathrm{H}$ & -1.59565400 & 1.25433100 & 6.84565500 \\
\hline $\mathrm{H}$ & -0.30411800 & 2.27457400 & 6.25904800 \\
\hline $\mathrm{H}$ & -1.35109200 & 1.45279700 & 5.12697300 \\
\hline $\mathrm{H}$ & -1.40937700 & -0.72594200 & 3.69542300 \\
\hline $\mathrm{H}$ & -0.10537900 & 0.29581500 & 3.13984300 \\
\hline $\mathrm{H}$ & 0.01476600 & -1.43978300 & 2.97756900 \\
\hline $\mathrm{H}$ & 0.97147500 & -3.50126600 & 4.22867000 \\
\hline $\mathrm{H}$ & 2.29151500 & -2.49000400 & 3.69209900 \\
\hline $\mathrm{H}$ & 2.46869900 & -3.46849400 & 5.12884200 \\
\hline $\mathrm{H}$ & 2.48474700 & -3.26041100 & 7.47021900 \\
\hline $\mathrm{H}$ & 3.63597300 & -1.96666700 & 7.23789700 \\
\hline $\mathrm{H}$ & 2.55569200 & -1.93876000 & 8.61095300 \\
\hline $\mathrm{H}$ & 5.06318900 & 3.09442200 & 9.95773700 \\
\hline $\mathrm{H}$ & 3.54000000 & 2.76897100 & 9.16587500 \\
\hline $\mathrm{H}$ & 4.77878400 & 1.53775800 & 9.21680800 \\
\hline $\mathrm{H}$ & 2.60988700 & 4.52534900 & 7.44047900 \\
\hline $\mathrm{H}$ & 3.63514300 & 5.55262500 & 8.41342300 \\
\hline $\mathrm{H}$ & 3.38919400 & 5.91429400 & 6.72174600 \\
\hline $\mathrm{H}$ & 4.45382600 & 5.44336300 & 4.39387400 \\
\hline $\mathrm{H}$ & 5.48419500 & 6.49469600 & 5.33520700 \\
\hline $\mathrm{H}$ & 6.18307000 & 5.42429700 & 4.14403700 \\
\hline $\mathrm{H}$ & 7.17870800 & 3.02358700 & 3.93677300 \\
\hline $\mathrm{H}$ & 8.25484500 & 4.05022200 & 4.85384300 \\
\hline $\mathrm{H}$ & 8.21800100 & 2.33002800 & 5.15821200 \\
\hline $\mathrm{H}$ & 6.88783900 & 0.52559400 & 6.84705800 \\
\hline $\mathrm{H}$ & 8.16235400 & 1.56356100 & 7.43976400 \\
\hline U & 6.87435100 & 1.06360000 & 8.50941500 \\
\hline
\end{tabular}

$\mathbf{T S}_{\mathrm{A} 4}$

$\mathrm{E}(\mathrm{RPBE}-\mathrm{PBE})=-1305.72667286$ A.U.

Thermal correction to Enthalpy $=0.388398$ A.U.

Entropy $($ Total $)=176.491 \mathrm{Cal} / \mathrm{Mol}-$ Kelvin

$\begin{array}{llll}\mathrm{Ru} & 4.47539900 & 2.08931900 & 6.15167600 \\ \mathrm{Ru} & 2.50355800 & 0.18104600 & 5.39077200 \\ \mathrm{Ru} & 2.66552700 & 2.58199300 & 3.59368200 \\ \mathrm{Ru} & 4.66474500 & 0.40581300 & 3.90496000 \\ \mathrm{O} & 4.45025300 & 0.15354900 & 5.88301900 \\ \mathrm{O} & 3.64370800 & 3.36056000 & 4.99264600 \\ \mathrm{C} & 5.72634200 & 1.57693200 & 7.99534200\end{array}$

\begin{tabular}{|c|c|c|c|}
\hline C & 4.52974500 & 2.24532700 & 8.37098200 \\
\hline $\mathrm{C}$ & 4.55116400 & 3.55561400 & 7.77422000 \\
\hline $\mathrm{C}$ & 5.74270500 & 3.66153200 & 6.99702700 \\
\hline $\mathrm{C}$ & 6.45673500 & 2.41736000 & 7.11075400 \\
\hline $\mathrm{C}$ & 1.26718600 & -1.49551300 & 5.97353800 \\
\hline $\mathrm{C}$ & 0.52478500 & -0.67710900 & 5.04328800 \\
\hline $\mathrm{C}$ & 0.34707300 & 0.60165200 & 5.64499900 \\
\hline $\mathrm{C}$ & 0.95532900 & 0.58119100 & 6.94284300 \\
\hline $\mathrm{C}$ & 1.51518300 & -0.71447900 & 7.14470800 \\
\hline $\mathrm{C}$ & 0.83725500 & 2.27402200 & 2.36935700 \\
\hline $\mathrm{C}$ & 1.95821600 & 2.29534100 & 1.50025500 \\
\hline $\mathrm{C}$ & 2.61756200 & 3.56202500 & 1.65996900 \\
\hline $\mathrm{C}$ & 1.86590800 & 4.34495600 & 2.59589100 \\
\hline $\mathrm{C}$ & 0.78808700 & 3.53018900 & 3.06827400 \\
\hline $\mathrm{C}$ & 6.76351700 & -0.13834800 & 3.54626900 \\
\hline $\mathrm{C}$ & 6.56947400 & 1.18170900 & 3.04198500 \\
\hline $\mathrm{C}$ & 5.57820900 & 1.11889800 & 2.01012500 \\
\hline $\mathrm{C}$ & 5.15519900 & -0.23344100 & 1.87213000 \\
\hline C & 5.88660400 & -1.01940800 & 2.83883800 \\
\hline $\mathrm{H}$ & 5.96135200 & 0.54305300 & 8.21324400 \\
\hline $\mathrm{H}$ & 3.75462100 & 1.85072000 & 9.01505000 \\
\hline $\mathrm{H}$ & 3.78407100 & 4.31228000 & 7.87299500 \\
\hline $\mathrm{H}$ & 6.03802000 & 4.51031000 & 6.39442100 \\
\hline $\mathrm{H}$ & 7.39922400 & 2.17659900 & 6.63630300 \\
\hline $\mathrm{H}$ & 1.55366400 & -2.52943400 & 5.82688400 \\
\hline $\mathrm{H}$ & 0.15449900 & -0.98767800 & 4.07482800 \\
\hline $\mathrm{H}$ & -0.15616200 & 1.45021000 & 5.20300000 \\
\hline $\mathrm{H}$ & 0.99343800 & 1.41091600 & 7.63620400 \\
\hline $\mathrm{H}$ & 2.06905300 & -1.04021100 & 8.01656000 \\
\hline $\mathrm{H}$ & 0.15260100 & 1.44677600 & 2.50323000 \\
\hline $\mathrm{H}$ & 2.28119000 & 1.48713500 & 0.85702100 \\
\hline $\mathrm{H}$ & 3.49890500 & 3.89194800 & 1.12295800 \\
\hline $\mathrm{H}$ & 2.10113700 & 5.34959400 & 2.92401500 \\
\hline $\mathrm{H}$ & 0.03888000 & 3.82515300 & 3.79320000 \\
\hline $\mathrm{H}$ & 7.43182300 & -0.42104900 & 4.35026400 \\
\hline $\mathrm{H}$ & 7.06519000 & 2.07943100 & 3.386436 \\
\hline $\mathrm{H}$ & 5.21243800 & 1.96164300 & 1.44058200 \\
\hline $\mathrm{H}$ & 4.43284500 & -0.60910100 & 1.15895300 \\
\hline $\mathrm{H}$ & 5.81222700 & -2.09061600 & 2.97884800 \\
\hline $\mathrm{H}$ & 3.29330300 & -0.71805400 & 4.07009300 \\
\hline $\mathrm{H}$ & 2.83819500 & 0.87101800 & 3.685819 \\
\hline
\end{tabular}

$\mathrm{TS}_{\mathrm{A} / \mathrm{A} \mathbf{A}}$

$\mathrm{E}(\mathrm{RPBE}-\mathrm{PBE})=1305.71011166 \mathrm{~A} . \mathrm{U}$.

Theion to Enthalpy= 0.387084 A.U.

Ent $=173.518 \mathrm{Cal} / \mathrm{Mol}-$ Kelvin

$\begin{array}{llll}\mathrm{Ru} & 4.64490900 & 2.06227900 & 6.31834400 \\ \mathrm{Ru} & 2.45741400 & 0.70573700 & 5.70716100 \\ \mathrm{Ru} & 2.42414200 & 2.07655400 & 3.21659500 \\ \mathrm{Ru} & 4.64090600 & 0.51353500 & 4.00349200 \\ \mathrm{O} & 4.41151600 & 0.13369600 & 5.89402600 \\ \mathrm{O} & 3.01883600 & 2.68731200 & 5.14447700 \\ \mathrm{C} & 5.75812800 & 1.90373600 & 8.18763400 \\ \mathrm{C} & 4.87257100 & 3.01783500 & 8.35663300 \\ \mathrm{C} & 5.19002400 & 3.98105700 & 7.36462800 \\ \mathrm{C} & 6.27637900 & 3.48898100 & 6.57506800 \\ \mathrm{C} & 6.64032300 & 2.19743000 & 7.09486700\end{array}$




$\begin{array}{rrrr}\mathrm{C} & 1.03528100 & -0.94439600 & 5.84377100 \\ \mathrm{C} & 0.31311200 & 0.27307800 & 5.56821400 \\ \mathrm{C} & 0.52682300 & 1.16228500 & 6.66413300 \\ \mathrm{C} & 1.36037000 & 0.50655900 & 7.62438400 \\ \mathrm{C} & 1.67388000 & -0.79095700 & 7.10935000 \\ \mathrm{C} & 1.99397800 & 1.81583800 & 1.12499500 \\ \mathrm{C} & 2.42198500 & 3.16728300 & 1.37553200 \\ \mathrm{C} & 1.47830600 & 3.77440300 & 2.27041900 \\ \mathrm{C} & 0.48436300 & 2.80354300 & 2.60017600 \\ \mathrm{C} & 0.81615300 & 1.58659800 & 1.89960300 \\ \mathrm{C} & 5.66460000 & -1.21299100 & 3.06211500 \\ \mathrm{C} & 6.62613100 & -0.24859100 & 3.49885100 \\ \mathrm{C} & 6.39191200 & 0.96961000 & 2.77313100 \\ \mathrm{C} & 5.30545600 & 0.75248300 & 1.87576600 \\ \mathrm{C} & 4.83457400 & -0.58125100 & 2.08297000 \\ \mathrm{H} & 5.76704600 & 1.00100800 & 8.78554500 \\ \mathrm{H} & 4.07874700 & 3.09787600 & 9.08881600 \\ \mathrm{H} & 4.65309800 & 4.90580100 & 7.19032300 \\ \mathrm{H} & 6.75621200 & 4.00802000 & 5.75561400 \\ \mathrm{H} & 7.44943900 & 1.57241000 & 6.73891800 \\ \mathrm{H} & 1.07539200 & -1.82247700 & 5.21213300 \\ \mathrm{H} & -0.29283900 & 0.47169200 & 4.69534300 \\ \mathrm{H} & 0.15063200 & 2.17482800 & 6.74161900 \\ \mathrm{H} & 1.69725600 & 0.91972600 & 8.56576700 \\ \mathrm{H} & 2.32876300 & -1.51548300 & 7.57741100 \\ \mathrm{H} & 2.47742200 & 1.09912000 & 0.47362800 \\ \mathrm{H} & 3.28926200 & 3.65413600 & 0.94613400 \\ \mathrm{H} & 1.53596200 & 4.78234700 & 2.66283900 \\ \mathrm{H} & -0.36627800 & 2.95651200 & 3.25286600 \\ \mathrm{H} & 0.25796100 & 0.65889800 & 1.93724000 \\ \mathrm{H} & 5.56845100 & -2.23099500 & 3.41797100 \\ \mathrm{H} & 7.39683800 & -0.40539000 & 4.24312100 \\ \mathrm{H} & 6.95463800 & 1.88869100 & 2.87543700 \\ \mathrm{H} & 4.90285900 & 1.47484600 & 1.18199400 \\ \mathrm{H} & 3.99552900 & -1.04079800 & 1.57613600 \\ & 2.78407300 & 0.39056800 & 3.89155400 \\ & 4.02744900 & 2.36839900 & 4.53170900\end{array}$

\section{A6}

$\mathrm{E}(\mathrm{RPBE}-\mathrm{PBE})=-1305.72403898$ A.U.

Thermal correction to Enthalpy $=0.393388$ A.U.

Entropy $($ Total $)=179.191 \mathrm{Cal} / \mathrm{Mol}-$ Kelvin

$\begin{array}{lrrr}\mathrm{Ru} & 4.72420300 & 2.20784000 & 6.74520900 \\ \mathrm{Ru} & 2.71176800 & 0.77197700 & 5.48435300 \\ \mathrm{Ru} & 2.32766400 & 1.98873700 & 3.13604100 \\ \mathrm{Ru} & 4.67279800 & 0.11911400 & 3.66781900 \\ \mathrm{O} & 4.74042800 & 0.53788800 & 5.55980500 \\ \mathrm{O} & 3.47782700 & 2.71476000 & 4.87272100 \\ \mathrm{C} & 5.22227100 & 2.05754600 & 8.79541400 \\ \mathrm{C} & 4.40741300 & 3.23588100 & 8.59913000 \\ \mathrm{C} & 5.09284100 & 4.09742900 & 7.69469200 \\ \mathrm{C} & 6.33522700 & 3.47035000 & 7.32424500 \\ \mathrm{C} & 6.41507600 & 2.21201100 & 8.01623900 \\ \mathrm{C} & 1.27658700 & -0.83560600 & 5.82556500 \\ \mathrm{C} & 0.53592300 & 0.37858400 & 5.62606700 \\ \mathrm{C} & 0.92641600 & 1.29390400 & 6.64675200 \\ \mathrm{C} & 1.87261000 & 0.64419900 & 7.51095900\end{array}$

$\begin{array}{lrrr}\mathrm{C} & 2.09166700 & -0.67401100 & 6.98447000 \\ \mathrm{C} & 1.72918100 & 1.74878400 & 1.10541300 \\ \mathrm{C} & 2.24024900 & 3.08382600 & 1.28165400 \\ \mathrm{C} & 1.42821700 & 3.73082900 & 2.26668300 \\ \mathrm{C} & 0.41771400 & 2.80879200 & 2.69779100 \\ \mathrm{C} & 0.59838600 & 1.57899400 & 1.96573900 \\ \mathrm{C} & 5.62329600 & -1.42866300 & 2.52866600 \\ \mathrm{C} & 6.62617200 & -0.49420400 & 2.97492600 \\ \mathrm{C} & 6.29747000 & 0.77594800 & 2.41704300 \\ \mathrm{C} & 5.12248000 & 0.61385700 & 1.59522700 \\ \mathrm{C} & 4.72793400 & -0.75476500 & 1.64136300 \\ \mathrm{H} & 4.98734700 & 1.21527000 & 9.43527700 \\ \mathrm{H} & 3.44308100 & 3.43156900 & 9.05166400 \\ \mathrm{H} & 4.72104400 & 5.03967000 & 7.31136800 \\ \mathrm{H} & 7.07829500 & 3.87723400 & 6.64956400 \\ \mathrm{H} & 7.22041200 & 1.49201100 & 7.93659600 \\ \mathrm{H} & 1.22959000 & -1.72127600 & 5.20472000 \\ \mathrm{H} & -0.18280900 & 0.56581400 & 4.84131400 \\ \mathrm{H} & 0.57423200 & 2.31287000 & 6.75068000 \\ \mathrm{H} & 2.30182600 & 1.05574100 & 8.41289500 \\ \mathrm{H} & 2.78377500 & -1.40926600 & 7.37595800 \\ \mathrm{H} & 2.12868900 & 0.99935400 & 0.43320000 \\ \mathrm{H} & 3.08466000 & 3.52254100 & 0.76450800 \\ \mathrm{H} & 1.57968300 & 4.73004300 & 2.65646300 \\ \mathrm{H} & -0.35237800 & 3.01168100 & 3.43213100 \\ \mathrm{H} & -0.01289500 & 0.68859300 & 2.04376300 \\ \mathrm{H} & 5.56479000 & -2.47402600 & 2.80797900 \\ \mathrm{H} & 7.44977200 & -0.71112900 & 3.64314000 \\ \mathrm{H} & 6.83923300 & 1.70137200 & 2.56970300 \\ \mathrm{H} & 3.64061500 & 1.39813600 & 1.03016500 \\ \mathrm{H} & 3.87044900 & -1.19434600 & 1.15005900 \\ & 2.84365100 & 0.24126000 & 3.71269600 \\ & 4.31510400 & 2.55425800 & 4.40063600\end{array}$

$\mathrm{TS}_{\mathrm{A} / \mathrm{A} \mathbf{A}}$

$\mathrm{E}(\mathrm{RPBE}-\mathrm{PBE})=-1305.71703571$ A.U.

Thermal correction to Enthalpy= 0.388945 A.U.

Entropy $($ Total $)=174.088 \mathrm{Cal} / \mathrm{Mol}-$ Kelvin

$\begin{array}{lrrr}\mathrm{Ru} & 4.05152900 & 2.64366500 & 6.35163900 \\ \mathrm{Ru} & 2.40361900 & 0.41889500 & 5.55961900 \\ \mathrm{Ru} & 2.65637100 & 2.46262400 & 3.78159600 \\ \mathrm{Ru} & 4.14379500 & 0.17734300 & 3.43038800 \\ \mathrm{O} & 4.36160900 & 1.01149600 & 5.18489300 \\ \mathrm{O} & 2.16480400 & 2.44061100 & 5.66902400 \\ \mathrm{C} & 5.32920900 & 2.78646900 & 8.09564300 \\ \mathrm{C} & 4.13957200 & 3.59362200 & 8.27427800 \\ \mathrm{C} & 4.13610800 & 4.59317600 & 7.25652100 \\ \mathrm{C} & 5.29887500 & 4.38589300 & 6.43456500 \\ \mathrm{C} & 6.05144000 & 3.29327100 & 6.98349000 \\ \mathrm{C} & 1.94266200 & -1.74782500 & 5.65219600 \\ \mathrm{C} & 0.71578800 & -1.00625300 & 5.56504900 \\ \mathrm{C} & 0.62205800 & -0.16485700 & 6.70726900 \\ \mathrm{C} & 1.79514200 & -0.36805100 & 7.51119800 \\ \mathrm{C} & 2.61223200 & -1.34168300 & 6.84574400 \\ \mathrm{C} & 1.63876200 & 2.65198900 & 1.85840900 \\ \mathrm{C} & 2.99764700 & 3.00596000 & 1.61947600 \\ \mathrm{C} & 3.32094000 & 4.10361000 & 2.47026200\end{array}$




$\begin{array}{rrrr}\mathrm{C} & 2.14302900 & 4.48112100 & 3.19729500 \\ \mathrm{C} & 1.09755900 & 3.56817800 & 2.82905500 \\ \mathrm{C} & 5.48274100 & -1.64405900 & 3.37337500 \\ \mathrm{C} & 6.31271600 & -0.49539200 & 3.44695700 \\ \mathrm{C} & 6.05151300 & 0.34553300 & 2.32318100 \\ \mathrm{C} & 5.05826800 & -0.30484900 & 1.52193500 \\ \mathrm{C} & 4.69741500 & -1.53377600 & 2.18212400 \\ \mathrm{H} & 5.61669600 & 1.93767200 & 8.70455200 \\ \mathrm{H} & 3.39780700 & 3.47511400 & 9.05438300 \\ \mathrm{H} & 3.37694100 & 5.35115800 & 7.10812500 \\ \mathrm{H} & 5.57872200 & 4.97490900 & 5.57011600 \\ \mathrm{H} & 6.96826700 & 2.88509100 & 6.57696400 \\ \mathrm{H} & 2.29693400 & -2.47695300 & 4.93654200 \\ \mathrm{H} & -0.00977800 & -1.07084700 & 4.76404000 \\ \mathrm{H} & -0.17616800 & 0.53706000 & 6.91297200 \\ \mathrm{H} & 2.01711300 & 0.11537000 & 8.45347600 \\ \mathrm{H} & 3.57473900 & -1.70052000 & 7.18878500 \\ \mathrm{H} & 1.10235300 & 1.83874000 & 1.38562600 \\ \mathrm{H} & 3.68061000 & 2.49843800 & 0.95333200 \\ \mathrm{H} & 4.29283100 & 4.57432700 & 2.55001200 \\ \mathrm{H} & 2.06544800 & 5.29763800 & 3.90363800 \\ \mathrm{H} & 0.07752200 & 3.57891600 & 3.19237200 \\ \mathrm{H} & 5.44034500 & -2.44911700 & 4.09531100 \\ \mathrm{H} & 6.95302000 & -0.24009900 & 4.28193300 \\ \mathrm{H} & 6.53241000 & 1.29016300 & 2.10468600 \\ \mathrm{H} & 4.68031800 & 0.04032000 & 0.56891000 \\ \mathrm{H} & 3.99011500 & -2.26702600 & 1.81790800 \\ \mathrm{H} & 2.18167500 & 0.68725900 & 3.82822200 \\ \mathrm{H} & 2.88666200 & -0.02294800 & 2.50137600\end{array}$

\section{A7}

$\mathrm{E}(\mathrm{RPBE}-\mathrm{PBE})=-1305.72585952 \mathrm{~A} . \mathrm{U}$.

Thermal correction to Enthalpy= 0.390556 A.U. Entropy $($ Total $)=177.598 \mathrm{Cal} / \mathrm{Mol}-$ Kelvin

$\begin{array}{lrrr}\mathrm{Ru} & 4.03191500 & 2.82074900 & 6.39080300 \\ \mathrm{Ru} & 2.35809700 & 0.46125200 & 5.43183700 \\ \mathrm{Ru} & 2.70349400 & 2.40773100 & 3.72604700 \\ \mathrm{Ru} & 4.07704900 & 0.10446800 & 3.37098900 \\ \mathrm{O} & 4.16982500 & 1.38158400 & 4.85600400 \\ \mathrm{O} & 2.15482300 & 2.47618200 & 5.69044300 \\ \mathrm{C} & 5.39470100 & 2.75868600 & 8.04412400 \\ \mathrm{C} & 4.14646500 & 3.37485100 & 8.43327400 \\ \mathrm{C} & 3.96093800 & 4.54431800 & 7.63091100 \\ \mathrm{C} & 5.08010200 & 4.62432400 & 6.72668600 \\ \mathrm{C} & 5.98656000 & 3.54548400 & 7.01522500 \\ \mathrm{C} & 2.21084700 & -1.77031100 & 5.70854500 \\ \mathrm{C} & 0.93075600 & -1.21218700 & 5.41161300 \\ \mathrm{C} & 0.59414200 & -0.27921700 & 6.44152900 \\ \mathrm{C} & 1.68716500 & -0.25731800 & 7.37995900 \\ \mathrm{C} & 2.68180000 & -1.16949500 & 6.91687200 \\ \mathrm{C} & 1.63922900 & 2.85109400 & 1.85062100 \\ \mathrm{C} & 3.03283600 & 2.95896800 & 1.56234000 \\ \mathrm{C} & 3.57770500 & 3.97796300 & 2.40286500 \\ \mathrm{C} & 2.51942000 & 4.51822400 & 3.19352400 \\ \mathrm{C} & 1.30945500 & 3.81453000 & 2.85450600 \\ \mathrm{C} & 5.49950800 & -1.62437300 & 3.50252600 \\ \mathrm{C} & 6.27471000 & -0.44853500 & 3.29605500\end{array}$

$\begin{array}{lrrc}\text { C } & 5.88463700 & 0.15949100 & 2.06711300 \\ \mathrm{C} & 4.85693700 & -0.65533800 & 1.49136800 \\ \mathrm{C} & 4.61820700 & -1.76102700 & 2.38367200 \\ \mathrm{H} & 5.80420100 & 1.84580700 & 8.45984600 \\ \mathrm{H} & 3.47302200 & 3.01652200 & 9.20222700 \\ \mathrm{H} & 3.12023000 & 5.22520900 & 7.67461200 \\ \mathrm{H} & 5.22897900 & 5.38750900 & 5.97185700 \\ \mathrm{H} & 6.91684800 & 3.33720200 & 6.50242400 \\ \mathrm{H} & 2.73641800 & -2.50443700 & 5.11438700 \\ \mathrm{H} & 0.32209900 & -1.45072500 & 4.54864800 \\ \mathrm{H} & -0.31820600 & 0.29917400 & 6.50553100 \\ \mathrm{H} & 1.75239900 & 0.35577600 & 8.26951000 \\ \mathrm{H} & 3.63757400 & -1.35986000 & 7.38897800 \\ \mathrm{H} & 0.95112100 & 2.15247800 & 1.39168300 \\ \mathrm{H} & 3.58006400 & 2.36330600 & 0.84533300 \\ \mathrm{H} & 4.61715300 & 4.27870000 & 2.44275600 \\ \mathrm{H} & 2.61317000 & 5.30807000 & 3.92737900 \\ \mathrm{H} & 0.32799600 & 3.98517400 & 3.27717200 \\ \mathrm{H} & 5.56815200 & -2.29324700 & 4.35062000 \\ \mathrm{H} & 6.98213400 & -0.03320200 & 4.00377100 \\ \mathrm{H} & 6.30150300 & 1.06300800 & 1.64179200 \\ \mathrm{H} & 4.37743200 & -0.49587600 & 0.53464400 \\ \mathrm{H} & 3.92294800 & -2.57329600 & 2.21797600 \\ \mathrm{H} & 1.73070700 & 0.96043400 & 3.87350800 \\ \mathrm{H} & 2.72271600 & -0.25845400 & 2.65435800\end{array}$

$\mathbf{T S}_{\text {A } 7 / \mathrm{A} 4}$

$\mathrm{E}(\mathrm{RPBE}-\mathrm{PBE})=-1305.71705441$ A.U.

Thermal correction to Enthalpy= 0.38891 A.U. Entropy $($ Total $)=174.768 \mathrm{Cal} / \mathrm{Mol}-$ Kelvin

$\begin{array}{lrrr}\mathrm{Ru} & 4.04173300 & 2.79083100 & 6.23688600 \\ \mathrm{Ru} & 2.39165900 & 0.48501700 & 5.48787400 \\ \mathrm{Ru} & 2.72150700 & 2.51852100 & 3.70064900 \\ \mathrm{Ru} & 4.18055300 & 0.18259400 & 3.42846100 \\ \mathrm{O} & 4.47502900 & 1.77135800 & 4.53169500 \\ \mathrm{O} & 2.14952300 & 2.41255900 & 5.65588800 \\ \mathrm{C} & 5.04330300 & 2.48329100 & 8.10456800 \\ \mathrm{C} & 3.92122400 & 3.36545200 & 8.29760400 \\ \mathrm{C} & 4.13697400 & 4.52735300 & 7.49690100 \\ \mathrm{C} & 5.40969200 & 4.36669700 & 6.82760400 \\ \mathrm{C} & 5.97754200 & 3.12743800 & 7.22596900 \\ \mathrm{C} & 2.29278000 & -1.75595600 & 5.71827000 \\ \mathrm{C} & 1.04627800 & -1.22758800 & 5.27540700 \\ \mathrm{C} & 0.56776700 & -0.30550800 & 6.27118200 \\ \mathrm{C} & 1.54105100 & -0.25433700 & 7.32539500 \\ \mathrm{C} & 2.62092200 & -1.12811600 & 6.95597200 \\ \mathrm{C} & 1.27642000 & 3.00193900 & 2.10054000 \\ \mathrm{C} & 2.59250100 & 2.91455200 & 1.52897800 \\ \mathrm{C} & 3.40771500 & 3.90543200 & 2.15370500 \\ \mathrm{C} & 2.59825300 & 4.62357700 & 3.09686000 \\ \mathrm{C} & 1.28178600 & 4.05225000 & 3.05817800 \\ \mathrm{C} & 5.80998400 & -1.32996100 & 3.62774000 \\ \mathrm{C} & 6.41068300 & -0.11524800 & 3.18618200 \\ \mathrm{C} & 5.84633000 & 0.25824500 & 1.93541100 \\ \mathrm{C} & 4.88165600 & -0.73978600 & 1.58009600 \\ \mathrm{C} & 4.87000900 & -1.72908200 & 2.62722300 \\ \mathrm{H} & 5.17618400 & 1.51399700 & 8.56885700\end{array}$




$\begin{array}{rrrr}\mathrm{H} & 3.05566100 & 3.16917600 & 8.91800400 \\ \mathrm{H} & 3.47644900 & 5.38189600 & 7.41909300 \\ \mathrm{H} & 5.85285700 & 5.07085600 & 6.13343600 \\ \mathrm{H} & 6.91156400 & 2.70921300 & 6.87259900 \\ \mathrm{H} & 2.91108600 & -2.46166400 & 5.18195000 \\ \mathrm{H} & 0.54193600 & -1.48038100 & 4.35097600 \\ \mathrm{H} & -0.36684700 & 0.24005600 & 6.23308500 \\ \mathrm{H} & 1.48322400 & 0.33969600 & 8.22818300 \\ \mathrm{H} & 3.52658600 & -1.29227600 & 7.52621600 \\ \mathrm{H} & 0.42999100 & 2.37579900 & 1.84847100 \\ \mathrm{H} & 2.90880300 & 2.21698600 & 0.76538700 \\ \mathrm{H} & 4.45771300 & 4.08244700 & 1.95682800 \\ \mathrm{H} & 2.91807700 & 5.45296900 & 3.71364800 \\ \mathrm{H} & 0.44665700 & 4.34214000 & 3.68354900 \\ \mathrm{H} & 6.03442400 & -1.85864300 & 4.54473000 \\ \mathrm{H} & 7.10292600 & 0.48402400 & 3.76407600 \\ \mathrm{H} & 6.09592500 & 1.14017000 & 1.36048700 \\ \mathrm{H} & 4.30406900 & -0.77396200 & 0.66596900 \\ \mathrm{H} & 4.27832000 & -2.63474800 & 2.63832400 \\ \mathrm{H} & 2.28818500 & 0.80197500 & 3.64839400 \\ \mathrm{H} & 2.87794900 & -0.57893200 & 2.97646100\end{array}$

$\mathrm{E}(\mathrm{RPBE}-\mathrm{PBE})=-1155.25747815$ A.U.

Thermal correction to Enthalpy= 0.298738 A.U.

$\begin{array}{lrrr}\mathrm{Ru} & 4.28711700 & 2.28970900 & 5.54058400 \\ \mathrm{Ru} & 2.60106100 & 0.62802500 & 5.70480000 \\ \mathrm{Ru} & 2.80351600 & 2.62873700 & 3.64422600 \\ \mathrm{Ru} & 4.84958200 & 0.55148200 & 3.91858500 \\ \mathrm{C} & 5.24388600 & 2.18748800 & 7.63062300 \\ \mathrm{C} & 4.05013000 & 2.97481400 & 7.69877100 \\ \mathrm{C} & 4.23974600 & 4.13869500 & 6.89416800 \\ \mathrm{C} & 5.52817100 & 4.03948600 & 6.29657200 \\ \mathrm{C} & 6.15826400 & 2.84321900 & 6.76561900 \\ \mathrm{C} & 1.60849100 & -1.28736800 & 5.34197800 \\ \mathrm{C} & 0.62069900 & -0.24642500 & 5.35828100 \\ \mathrm{C} & 0.64574100 & 0.37003400 & 6.64642200 \\ \mathrm{C} & 1.65742400 & -0.28896900 & 7.42350300 \\ \mathrm{C} & 2.25041500 & -1.31927200 & 6.61911900 \\ \mathrm{C} & 1.37008600 & 2.31563600 & 2.00064400 \\ \mathrm{C} & 2.49783900 & 3.04286500 & 1.51160300 \\ \mathrm{C} & 2.58591600 & 4.27293400 & 2.24596200 \\ \mathrm{C} & 1.51120400 & 4.29690100 & 3.19329900 \\ \mathrm{C} & 0.76315200 & 3.08118500 & 3.04875300 \\ \mathrm{C} & 5.77211500 & -1.39996400 & 3.91377100 \\ \mathrm{C} & 6.75242600 & -0.40471900 & 3.57262600 \\ \mathrm{C} & 6.33554700 & 0.22822600 & 2.35777300 \\ \mathrm{C} & 5.10583900 & -0.37560700 & 1.94112600 \\ \mathrm{C} & 4.76186800 & -1.38109400 & 2.89915300 \\ \mathrm{H} & 5.40836100 & 1.24326800 & 8.13269200 \\ \mathrm{H} & 3.17537800 & 2.74966300 & 8.29358800 \\ \mathrm{H} & 3.52044000 & 4.93040900 & 6.73582500 \\ \mathrm{H} & 5.95386600 & 4.74147500 & 5.59060100 \\ \mathrm{H} & 7.14453400 & 2.49409000 & 6.49168800 \\ \mathrm{H} & 1.83822700 & -1.92695200 & 4.50063800 \\ \mathrm{H} & -0.02153800 & 0.03009000 & 4.53291500 \\ \mathrm{H} & 0.01886500 & 1.18933100 & 6.97466100\end{array}$

$\begin{array}{cccc}\mathrm{H} & 1.92775500 & -0.05006200 & 8.44534600 \\ \mathrm{H} & 3.03705700 & -1.99746700 & 6.92288800 \\ \mathrm{H} & 1.05160500 & 1.33810600 & 1.66214500 \\ \mathrm{H} & 3.17313400 & 2.72244400 & 0.72923700 \\ \mathrm{H} & 3.33231300 & 5.04464300 & 2.10628000 \\ \mathrm{H} & 1.30605000 & 5.08649400 & 3.90579500 \\ \mathrm{H} & -0.11104400 & 2.80190300 & 3.62131700 \\ \mathrm{H} & 5.79641900 & -2.05091700 & 4.77801500 \\ \mathrm{H} & 7.64605600 & -0.17200000 & 4.13815300 \\ \mathrm{H} & 6.85317200 & 1.03150900 & 1.84890400 \\ \mathrm{H} & 4.53101600 & -0.11006500 & 1.06395300 \\ \mathrm{H} & 3.88069400 & -2.00805600 & 2.86879200 \\ \mathrm{H} & 2.93713300 & 0.76332400 & 3.88681300 \\ \mathrm{H} & 4.68303700 & 2.42326700 & 3.73964900\end{array}$

\section{$\mathbf{8 a}$}

$\mathrm{E}(\mathrm{RPBE}-\mathrm{PBE})=-1548.45498728$ A.U.

Thermal correction to Enthalpy=0.561442 A.U

\begin{tabular}{|c|c|c|c|}
\hline $\mathrm{Ru}$ & 4.24019000 & 2.32189100 & 6.02205300 \\
\hline $\mathrm{Ru}$ & 2.63790300 & 0.57317900 & 5.39756700 \\
\hline $\mathrm{Ru}$ & 2.55158900 & 2.18277500 & 3.67799200 \\
\hline $\mathrm{Ru}$ & 4.55752700 & 0.09240800 & 3.97655600 \\
\hline $\mathrm{C}$ & 5.83160800 & 2.66466400 & 7.47751500 \\
\hline $\mathrm{C}$ & 4.54687500 & 3.02035700 & 8.02604800 \\
\hline $\mathrm{C}$ & 4.00056900 & 4.08731400 & 7.21662600 \\
\hline $\mathrm{C}$ & 4.95694800 & 4.39074500 & 6.18362300 \\
\hline C & 6.08509700 & 3.51425100 & 6.34552100 \\
\hline $\mathrm{C}$ & 1.22859800 & -1.24034800 & 5.29890700 \\
\hline $\mathrm{C}$ & 0.45976500 & -0.05159200 & 5.11329000 \\
\hline $\mathrm{C}$ & 0.52795800 & 0.71445600 & 6.31973900 \\
\hline $\mathrm{C}$ & 1.35952000 & 0.02086000 & 7.23745600 \\
\hline $\mathrm{C}$ & 1.80301700 & -1.18042100 & 6.59877400 \\
\hline $\mathrm{C}$ & 1.51591100 & 2.38161300 & 1.76397300 \\
\hline $\mathrm{C}$ & 2.83041400 & 2.92148000 & 1.62442000 \\
\hline $\mathrm{C}$ & 2.96285100 & 4.01587600 & 2.53947800 \\
\hline $\mathrm{C}$ & 1.73087700 & 4.15901700 & 3.25217100 \\
\hline $\mathrm{C}$ & 0.83690500 & 3.14487200 & 2.77274700 \\
\hline $\mathrm{C}$ & 5.71137900 & -1.72757500 & 3.88696800 \\
\hline $\mathrm{C}$ & 6.63126900 & -0.61804500 & 3.80280100 \\
\hline $\mathrm{C}$ & 6.31537600 & 0.13764400 & 2.62291200 \\
\hline $\mathrm{C}$ & 5.19671600 & -0.49345700 & 1.97405500 \\
\hline $\mathrm{C}$ & 4.82537200 & -1.64966200 & 2.75272500 \\
\hline $\mathrm{C}$ & 4.87638600 & 5.52907500 & 5.21667300 \\
\hline $\mathrm{C}$ & 2.72578000 & 4.82409300 & 7.48395200 \\
\hline $\mathrm{C}$ & 3.94309400 & 2.47146900 & 9.28125900 \\
\hline $\mathrm{C}$ & 6.78066300 & 1.66701700 & 8.06404800 \\
\hline $\mathrm{C}$ & 7.35561900 & 3.58648900 & 5.55971400 \\
\hline $\mathrm{C}$ & 7.80300200 & -0.40260900 & 4.70591100 \\
\hline $\mathrm{C}$ & 5.75920600 & -2.82682100 & 4.90289200 \\
\hline $\mathrm{C}$ & 3.77704800 & -2.64837300 & 2.37315300 \\
\hline $\mathrm{C}$ & 4.63368700 & -0.14790900 & 0.63151800 \\
\hline $\mathrm{C}$ & 7.07171400 & 1.32045900 & 2.10417300 \\
\hline $\mathrm{H}$ & 4.36042300 & 2.01228600 & 4.06851200 \\
\hline $\mathrm{H}$ & 4.39546200 & 0.42672100 & 5.86370300 \\
\hline $\mathrm{H}$ & 1.37813600 & -2.02278100 & 4.56811700 \\
\hline $\mathrm{H}$ & -0.11898000 & 0.19879800 & 4.23465400 \\
\hline $\mathrm{H}$ & 0.04500000 & 1.66744600 & 6.49146600 \\
\hline
\end{tabular}




$\begin{array}{crrr}\text { H } & 1.62428800 & 0.34504500 & 8.23453500 \\ \mathrm{H} & 2.48045100 & -1.90891600 & 7.02624100 \\ \mathrm{H} & 1.10634500 & 1.54750500 & 1.20854600 \\ \mathrm{H} & 3.59383000 & 2.56499800 & 0.94841400 \\ \mathrm{H} & 3.84977800 & 4.61730000 & 2.68007000 \\ \mathrm{H} & 1.50482500 & 4.90369600 & 4.00315900 \\ \mathrm{H} & -0.17762900 & 2.98242900 & 3.11743800 \\ \mathrm{H} & 3.84307500 & 5.75189500 & 4.93644500 \\ \mathrm{H} & 5.30397800 & 6.44202800 & 5.65682000 \\ \mathrm{H} & 5.43477200 & 5.31554800 & 4.29990200 \\ \mathrm{H} & 2.89299300 & 5.66061400 & 8.17899100 \\ \mathrm{H} & 2.30284900 & 5.23445900 & 6.56266800 \\ \mathrm{H} & 1.97134400 & 4.16669800 & 7.92659600 \\ \mathrm{H} & 4.28045800 & 3.04050000 & 10.16050600 \\ \mathrm{H} & 2.85019900 & 2.52272000 & 9.25472400 \\ \mathrm{H} & 4.22255800 & 1.42467100 & 9.43632800 \\ \mathrm{H} & 7.40465600 & 2.13080900 & 8.84193000 \\ \mathrm{H} & 6.24641600 & 0.82895900 & 8.52229800 \\ \mathrm{H} & 7.44890800 & 1.25485500 & 7.30317200 \\ \mathrm{H} & 8.01295700 & 4.37667000 & 5.95188400 \\ \mathrm{H} & 7.91044700 & 2.64613400 & 5.61022100 \\ \mathrm{H} & 7.16630800 & 3.80732600 & 4.50474800 \\ \mathrm{H} & 7.58039800 & -0.70840000 & 5.73241600 \\ \mathrm{H} & 8.66917600 & -0.98859600 & 4.36482100 \\ \mathrm{H} & 8.10408600 & 0.64770300 & 4.72733500 \\ \mathrm{H} & 6.46098400 & -3.61642400 & 4.59608500 \\ \mathrm{H} & 6.08385000 & -2.45413400 & 5.87938000 \\ \mathrm{H} & 4.77783800 & -3.29141500 & 5.03849200 \\ \mathrm{H} & 4.19091400 & -3.40879400 & 1.69405100 \\ \mathrm{H} & 3.38255700 & -3.16951000 & 3.25047400 \\ \mathrm{H} & 2.93516000 & -2.16938200 & 1.86384900 \\ \mathrm{H} & 5.04048400 & -0.81656700 & -0.14139300 \\ \mathrm{H} & 3.54364800 & -0.24100500 & 0.61349400 \\ & 4.88851900 & 0.87462500 & 0.33994600 \\ \mathrm{H} & 7.84698700 & 1.01007700 & 1.38842800 \\ \mathrm{H} & 7.56616600 & 1.86449700 & 2.91347400\end{array}$

8b

$\mathrm{E}(\mathrm{RPBE}-\mathrm{PBE})=-1548.45992102$ A.U.

Thermal correction to Enthalpy= 0.565455 A.U.

$\begin{array}{lrrr}\mathrm{Ru} & 4.10131600 & 2.77413800 & 5.65113300 \\ \mathrm{Ru} & 2.35579900 & 0.44349600 & 5.40077400 \\ \mathrm{Ru} & 2.55707000 & 2.21108600 & 3.68541500 \\ \mathrm{Ru} & 4.33206000 & -0.09599300 & 3.93014400 \\ \mathrm{C} & 5.47487400 & 2.68704100 & 7.32723400 \\ \mathrm{C} & 4.20534000 & 3.22079200 & 7.74759600 \\ \mathrm{C} & 3.95090200 & 4.42631400 & 6.99296800 \\ \mathrm{C} & 5.07485100 & 4.64505300 & 6.12242000 \\ \mathrm{C} & 6.00637000 & 3.56511800 & 6.31471700 \\ \mathrm{C} & 1.38323000 & -1.50952300 & 5.69028600 \\ \mathrm{C} & 0.56929400 & -0.50883800 & 6.30604000 \\ \mathrm{C} & 1.34853000 & 0.09397600 & 7.33943100 \\ \mathrm{C} & 2.62237300 & -0.55461400 & 7.38841700 \\ \mathrm{C} & 2.64254900 & -1.55377100 & 6.37280000 \\ \mathrm{C} & 2.14947100 & 2.57551400 & 1.54987800 \\ \mathrm{C} & 3.33757600 & 3.29394900 & 1.88646300\end{array}$

\begin{tabular}{|c|c|c|c|}
\hline & 3.00055500 & 4.25014700 & 2.88797900 \\
\hline & 1.60099800 & 4.12033800 & 3.16864000 \\
\hline$C_{1}$ & 1.06679300 & 3.09813300 & 2.32635200 \\
\hline & 5.55406300 & -1.86968200 & 3.76061500 \\
\hline & 6.40031200 & -0.70756400 & 3.82099500 \\
\hline & 6.08850700 & 0.13484600 & 2.69185000 \\
\hline & 5.04090700 & -0.50770800 & 1.94119800 \\
\hline & 4.70056400 & -1.74205300 & 2.60987800 \\
\hline & 5.30509100 & 5.84182700 & 5.25453800 \\
\hline & 2.77912600 & 5.33951000 & 7.17583300 \\
\hline & 3.36759800 & 2.72923300 & 8.88350100 \\
\hline & 6.18726600 & 1.52582000 & 7.94208400 \\
\hline & 7.37003900 & 3.49577000 & 5.70485500 \\
\hline & 7.52553700 & -0.51376800 & 4.78491100 \\
\hline & 5.62823000 & -3.04316700 & 4.68571900 \\
\hline & 3.71233100 & -2.75564400 & 2.12297000 \\
\hline & 4.49170200 & -0.07144900 & 0.62122300 \\
\hline & 6.82330300 & 1.37940200 & 2.31016700 \\
\hline & 2.40540700 & 0.39329000 & 3.60484500 \\
\hline & 2.17277400 & 2.25862700 & 5.44252600 \\
\hline & 1.09255900 & -2.13446300 & 4.85536700 \\
\hline & -0.43804600 & -0.23683800 & 6.01942900 \\
\hline & 1.02997800 & 0.91191300 & 7.97209300 \\
\hline & 3.42570600 & -0.33222100 & 8.07495900 \\
\hline & 3.45490300 & -2.23409400 & 6.16632200 \\
\hline & 2.07944100 & 1.76747600 & 0.83374800 \\
\hline & 4.31944100 & 3.14471200 & 1.46080600 \\
\hline & 3.67580300 & 4.96349700 & 3.33437900 \\
\hline & 1.04274700 & 4.69872500 & 3.89378400 \\
\hline & 0.03869200 & 2.76138700 & 2.30152900 \\
\hline & 4.36800300 & 6.34578300 & 4.99740900 \\
\hline & 5.93641400 & 6.57803100 & 5.77300800 \\
\hline & 5.81357300 & 5.57571600 & 4.32150000 \\
\hline & 2.97154300 & 6.07328000 & 7.97235800 \\
\hline & 2.55591800 & 5.89634100 & 6.26015500 \\
\hline & 1.87821300 & 4.78074300 & 7.44725500 \\
\hline & 3.62153600 & 3.27048300 & 9.80685900 \\
\hline & 2.30121000 & 2.88378700 & 8.69409500 \\
\hline & 3.52414500 & 1.66478100 & 9.07425400 \\
\hline & 6.74303800 & 1.84004700 & 8.83830900 \\
\hline & 5.49170200 & 0.73737700 & 8.24100000 \\
\hline & 6.90243400 & 1.08307800 & 7.24491600 \\
\hline & 8.08353700 & 4.09870700 & 6.28632400 \\
\hline $\mathrm{H}$ & 7.75067900 & 2.47179900 & 5.68328600 \\
\hline & 7.37661000 & 3.87938500 & 4.67962400 \\
\hline $\mathrm{H}$ & 7.26328000 & -0.86707100 & 5.78679400 \\
\hline & 8.41666300 & -1.07032100 & 4.45794300 \\
\hline $\mathrm{H}$ & 7.80589600 & 0.53930400 & 4.86007700 \\
\hline & 6.41027300 & -3.74475700 & 4.36078100 \\
\hline & 5.86962600 & -2.73637500 & 5.70927800 \\
\hline & 4.68613300 & -3.60001900 & 4.71449500 \\
\hline & 4.17815900 & -3.44610300 & 1.40457000 \\
\hline & 3.31337400 & -3.35655300 & 2.94627300 \\
\hline & 2.86341600 & -2.27831800 & 1.62373000 \\
\hline & 5.04270100 & -0.55112200 & -0.20108200 \\
\hline & 3.43747100 & -0.34573700 & 0.51567900 \\
\hline & 4.57112000 & 1.00990100 & 0.48447100 \\
\hline
\end{tabular}




$\begin{array}{llll}\mathrm{H} & 7.77208900 & 1.13776000 & 1.80811800 \\ \mathrm{H} & 6.23522300 & 1.99652100 & 1.62556800 \\ \mathrm{H} & 7.04670700 & 1.98897000 & 3.18948800\end{array}$

$8 c$

$\mathrm{E}(\mathrm{RPBE}-\mathrm{PBE})=-1548.46058242$ A.U.

Thermal correction to Enthalpy= 0.563447 A.U.

\begin{tabular}{|c|c|c|c|}
\hline $\mathrm{Ru}$ & 4.17545700 & 2.43753200 & 6.08147200 \\
\hline $\mathrm{Ru}$ & 2.51475600 & 0.65612900 & 5.35975000 \\
\hline $\mathrm{Ru}$ & 2.53101100 & 2.23292000 & 3.54282000 \\
\hline $\mathrm{Ru}$ & 4.44689800 & 0.02824200 & 3.96012200 \\
\hline $\mathrm{C}$ & 5.81381200 & 2.71329500 & 7.47062400 \\
\hline $\mathrm{C}$ & 4.54838300 & 2.96211100 & 8.11749700 \\
\hline C & 3.91951100 & 4.06732400 & 7.43466500 \\
\hline C & 4.80217700 & 4.50397200 & 6.38093200 \\
\hline $\mathrm{C}$ & 5.96845900 & 3.66392800 & 6.39870600 \\
\hline $\mathrm{C}$ & 1.40838200 & -1.29826000 & 5.10732300 \\
\hline $\mathrm{C}$ & 0.47948300 & -0.22198600 & 4.97470800 \\
\hline $\mathrm{C}$ & 0.43615900 & 0.46386400 & 6.23248100 \\
\hline $\mathrm{C}$ & 1.30510200 & -0.19486600 & 7.14567000 \\
\hline $\mathrm{C}$ & 1.92743500 & -1.26232700 & 6.44061900 \\
\hline $\mathrm{C}$ & 1.10784000 & 3.05438100 & 2.13773200 \\
\hline $\mathrm{C}$ & 2.15038000 & 2.37050200 & 1.42401500 \\
\hline $\mathrm{C}$ & 3.38479400 & 3.03149100 & 1.72123100 \\
\hline $\mathrm{C}$ & 3.10508400 & 4.11845700 & 2.60898400 \\
\hline $\mathrm{C}$ & 1.69732600 & 4.13985000 & 2.85708500 \\
\hline $\mathrm{C}$ & 5.69489400 & -1.73575900 & 3.93760200 \\
\hline $\mathrm{C}$ & 6.54054400 & -0.57769300 & 3.84555900 \\
\hline $\mathrm{C}$ & 6.19025500 & 0.14651200 & 2.65173700 \\
\hline $\mathrm{C}$ & 5.12856000 & -0.57106900 & 1.99256900 \\
\hline $\mathrm{C}$ & 4.81583900 & -1.72733200 & 2.79496400 \\
\hline $\mathrm{C}$ & 4.61111000 & 5.72136700 & 5.53347700 \\
\hline $\mathrm{C}$ & 2.62565200 & 4.71648800 & 7.81714700 \\
\hline $\mathrm{C}$ & 4.04191700 & 2.27468500 & 9.34665900 \\
\hline $\mathrm{C}$ & 6.83562600 & 1.72317500 & 7.93364900 \\
\hline $\mathrm{C}$ & 7.18221100 & 3.85524000 & 5.54505500 \\
\hline $\mathrm{C}$ & 7.68815900 & -0.26876500 & 4.75157800 \\
\hline $\mathrm{C}$ & 5.77439000 & -2.80182400 & 4.98496800 \\
\hline $\mathrm{C}$ & 3.82833400 & -2.79200800 & 2.43449600 \\
\hline $\mathrm{C}$ & 4.56961800 & -0.29176500 & 0.63377900 \\
\hline $\mathrm{C}$ & 6.91888700 & 1.34239400 & 2.12727500 \\
\hline $\mathrm{H}$ & 4.27306300 & 0.55392800 & 5.81637000 \\
\hline $\mathrm{H}$ & 2.44626100 & 2.50229100 & 5.40552100 \\
\hline $\mathrm{H}$ & 1.65535300 & -2.02770600 & 4.34957800 \\
\hline $\mathrm{H}$ & -0.10911600 & 0.01549400 & 4.09980300 \\
\hline $\mathrm{H}$ & -0.15205500 & 1.34796300 & 6.44450100 \\
\hline $\mathrm{H}$ & 1.50217500 & 0.09971900 & 8.16742100 \\
\hline $\mathrm{H}$ & 2.68121400 & -1.92954300 & 6.83896100 \\
\hline $\mathrm{H}$ & 0.05698100 & 2.79152300 & 2.13404600 \\
\hline $\mathrm{H}$ & 2.02125600 & 1.51178800 & 0.77941100 \\
\hline $\mathrm{H}$ & 4.36252900 & 2.76568700 & 1.35083700 \\
\hline $\mathrm{H}$ & 3.83608500 & 4.79591000 & 3.02394300 \\
\hline $\mathrm{H}$ & 1.17412300 & 4.82889700 & 3.50770300 \\
\hline $\mathrm{H}$ & 5.17660700 & 5.65393500 & 4.59910500 \\
\hline $\mathrm{H}$ & 3.55847600 & 5.88011000 & 5.28006300 \\
\hline $\mathrm{H}$ & 4.96241200 & 6.61990200 & 6.06222200 \\
\hline $\mathrm{H}$ & 2.78981500 & 5.52084100 & 8.54915800 \\
\hline
\end{tabular}

$\begin{array}{lrrr}\mathrm{H} & 2.12723600 & 5.15385400 & 6.94658500 \\ \mathrm{H} & 1.93395000 & 3.99493700 & 8.26254700 \\ \mathrm{H} & 4.44024700 & 2.74910800 & 10.25609700 \\ \mathrm{H} & 2.94989000 & 2.31251800 & 9.40442700 \\ \mathrm{H} & 4.33729000 & 1.22104700 & 9.36272000 \\ \mathrm{H} & 7.45057400 & 2.14843400 & 8.74031500 \\ \mathrm{H} & 6.36567300 & 0.81306500 & 8.31984000 \\ \mathrm{H} & 7.50837800 & 1.43057700 & 7.12412000 \\ \mathrm{H} & 7.83532000 & 4.63645600 & 5.96209600 \\ \mathrm{H} & 7.77431900 & 2.93888500 & 5.47527900 \\ \mathrm{H} & 6.91515600 & 4.15514100 & 4.52668100 \\ \mathrm{H} & 7.47658800 & -0.55787200 & 5.78491100 \\ \mathrm{H} & 8.58847800 & -0.81377100 & 4.43107700 \\ \mathrm{H} & 7.92984900 & 0.79733300 & 4.74247200 \\ \mathrm{H} & 6.49742200 & -3.58095700 & 4.70185100 \\ \mathrm{H} & 6.08877200 & -2.39135000 & 5.94972000 \\ \mathrm{H} & 4.80617500 & -3.29011200 & 5.13412700 \\ \mathrm{H} & 4.28213500 & -3.53239100 & 1.75943600 \\ \mathrm{H} & 3.47590900 & -3.33080800 & 3.31967600 \\ \mathrm{H} & 2.95399700 & -2.37215600 & 1.92698700 \\ \mathrm{H} & 5.13601600 & -0.83790900 & -0.13524600 \\ \mathrm{H} & 3.52378000 & -0.60413000 & 0.55608800 \\ \mathrm{H} & 4.61774400 & 0.77023100 & 0.38188300 \\ \mathrm{H} & 7.86250200 & 1.04466100 & 1.64636000 \\ \mathrm{H} & 6.32911100 & 1.87821700 & 1.37906700 \\ \mathrm{H} & 7.15684700 & 2.04905500 & 2.92738600\end{array}$




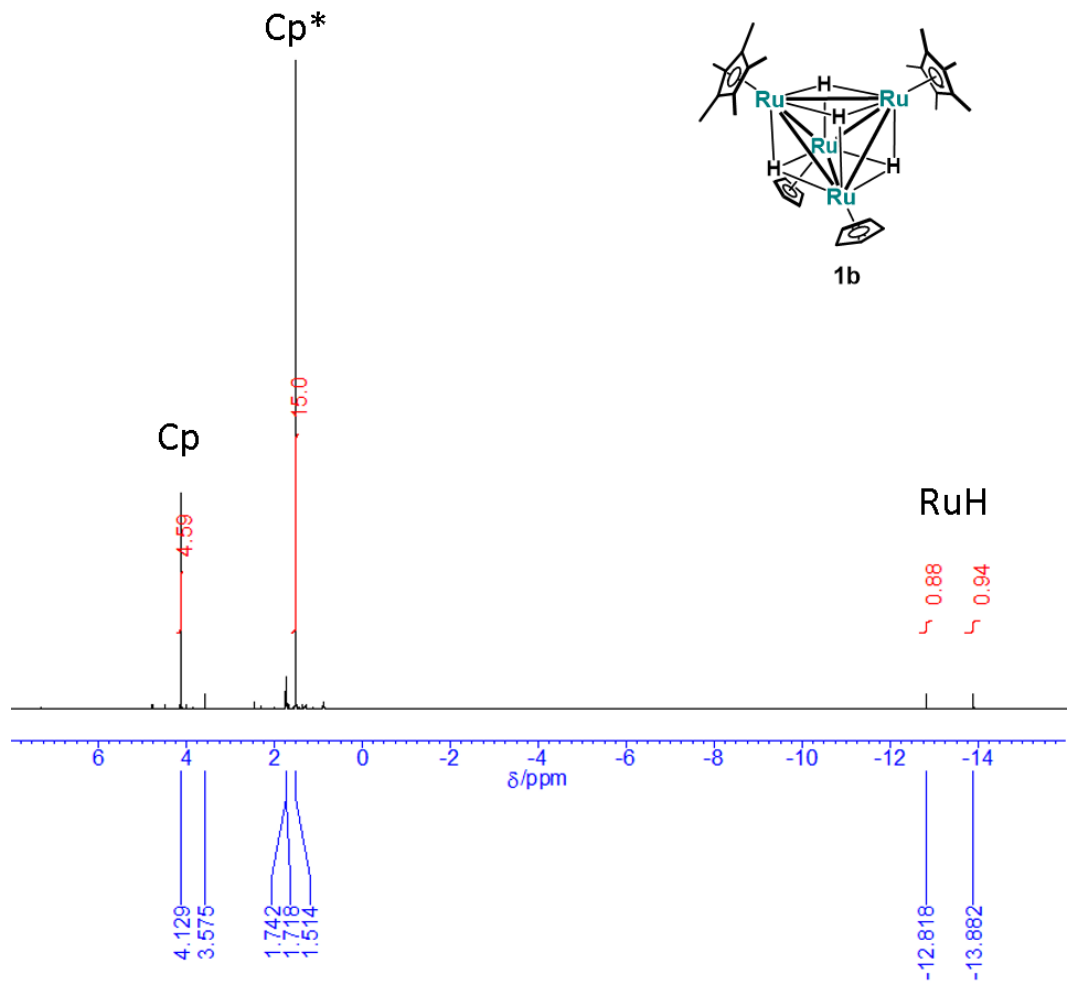

Figure S20 ${ }^{1} \mathrm{H}$ NMR spectrum of $\mathbf{1 b}\left(400 \mathrm{MHz},\left[\mathrm{D}_{8}\right]\right.$ tetrahydrofuran, $\left.25^{\circ} \mathrm{C}\right)$.

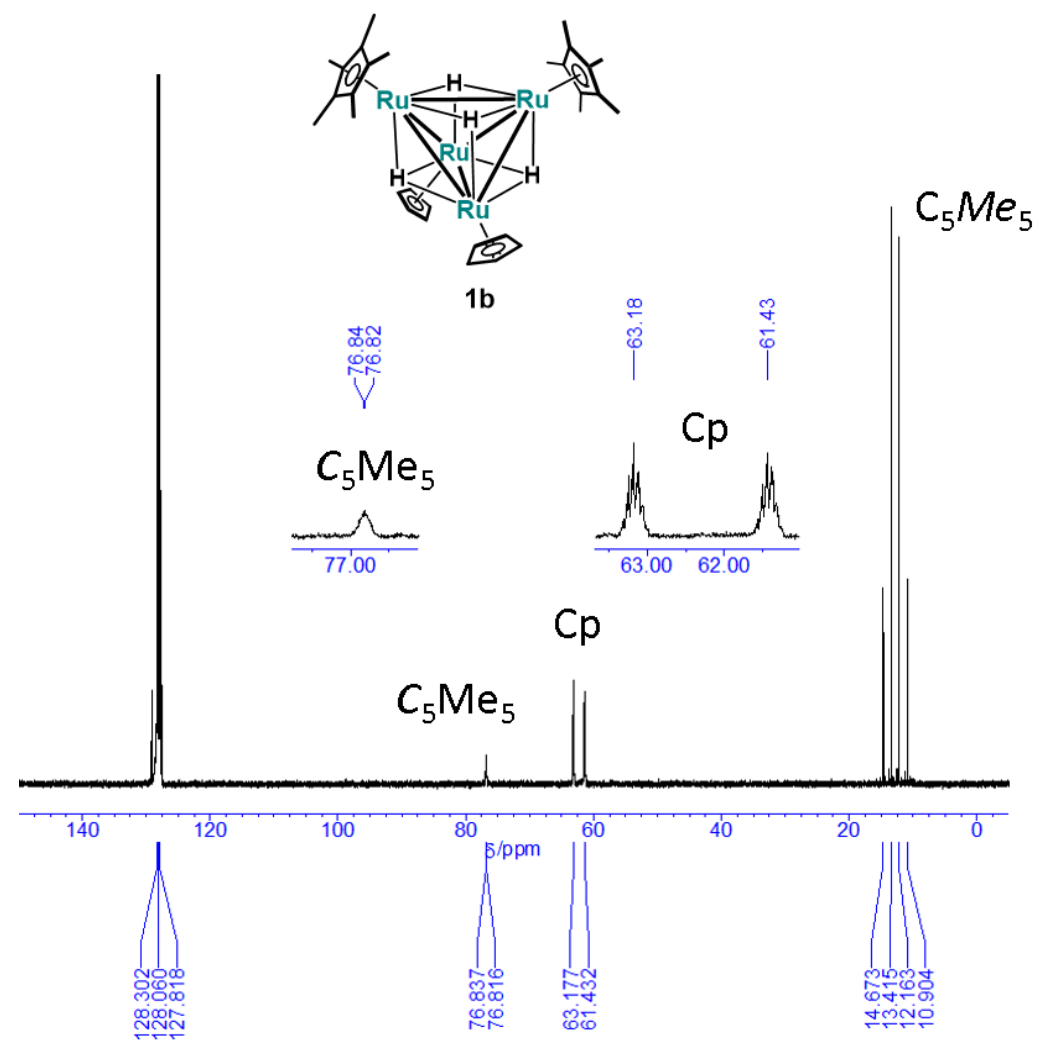

Figure S21 ${ }^{13} \mathrm{C}$ NMR spectrum of $\mathbf{1 b}\left(100 \mathrm{MHz},\left[\mathrm{D}_{6}\right]\right.$ benzene, $\left.25^{\circ} \mathrm{C}\right)$. 


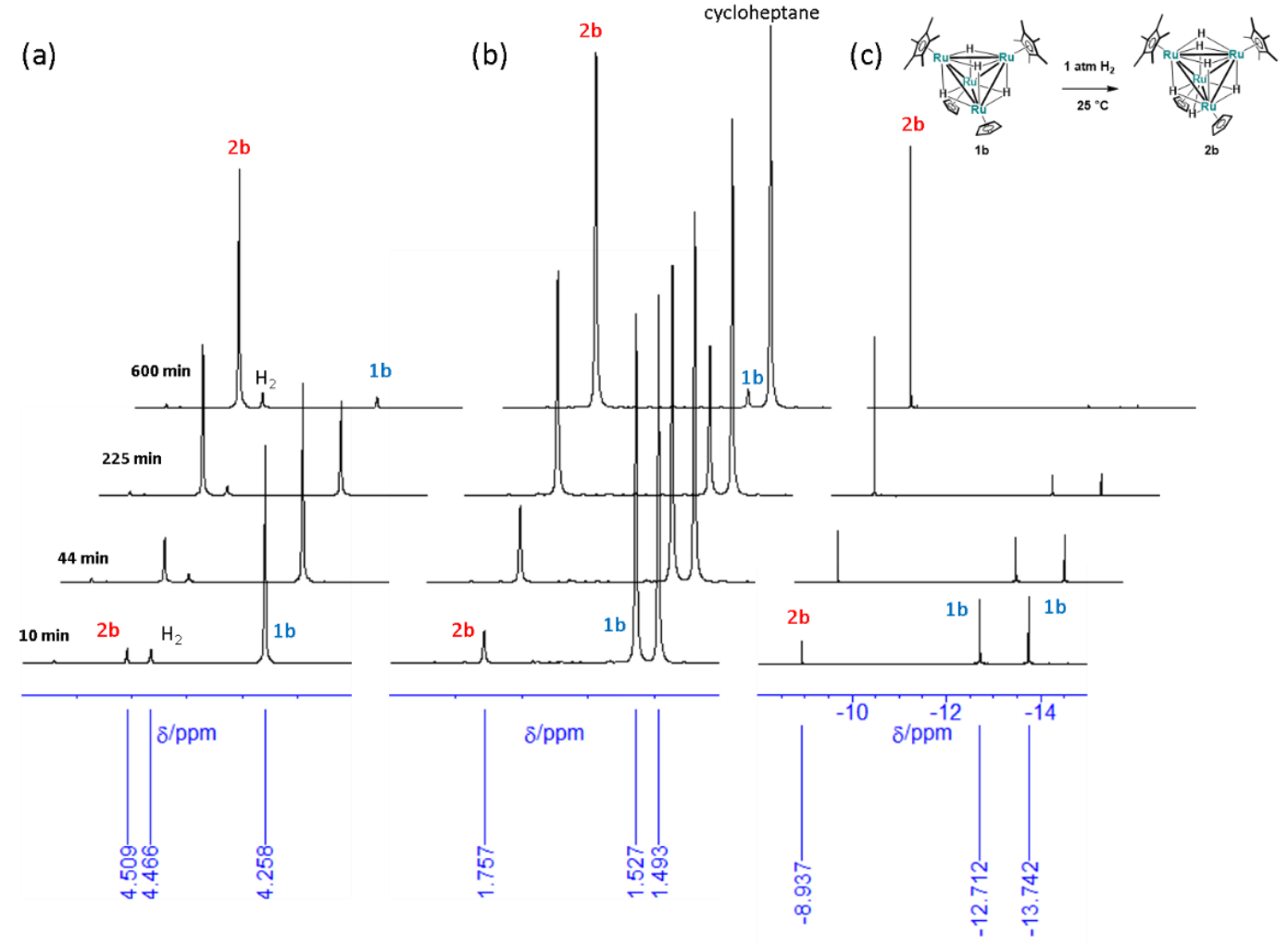

Figure S22 ${ }^{1} \mathrm{H}$ NMR spectra of the hydrogenation of $\mathbf{1 b}$ recorded at given time, showing (a) $\mathrm{Cp}$, (b) $\mathrm{Cp}$ *, and (c) hydrido regions $\left(400 \mathrm{MHz},\left[\mathrm{D}_{8}\right]\right.$ benzene, $25^{\circ} \mathrm{C}$ ). The singlet resonating at $\delta 1.49$ was derived from cycloheptane used as an internal standard.

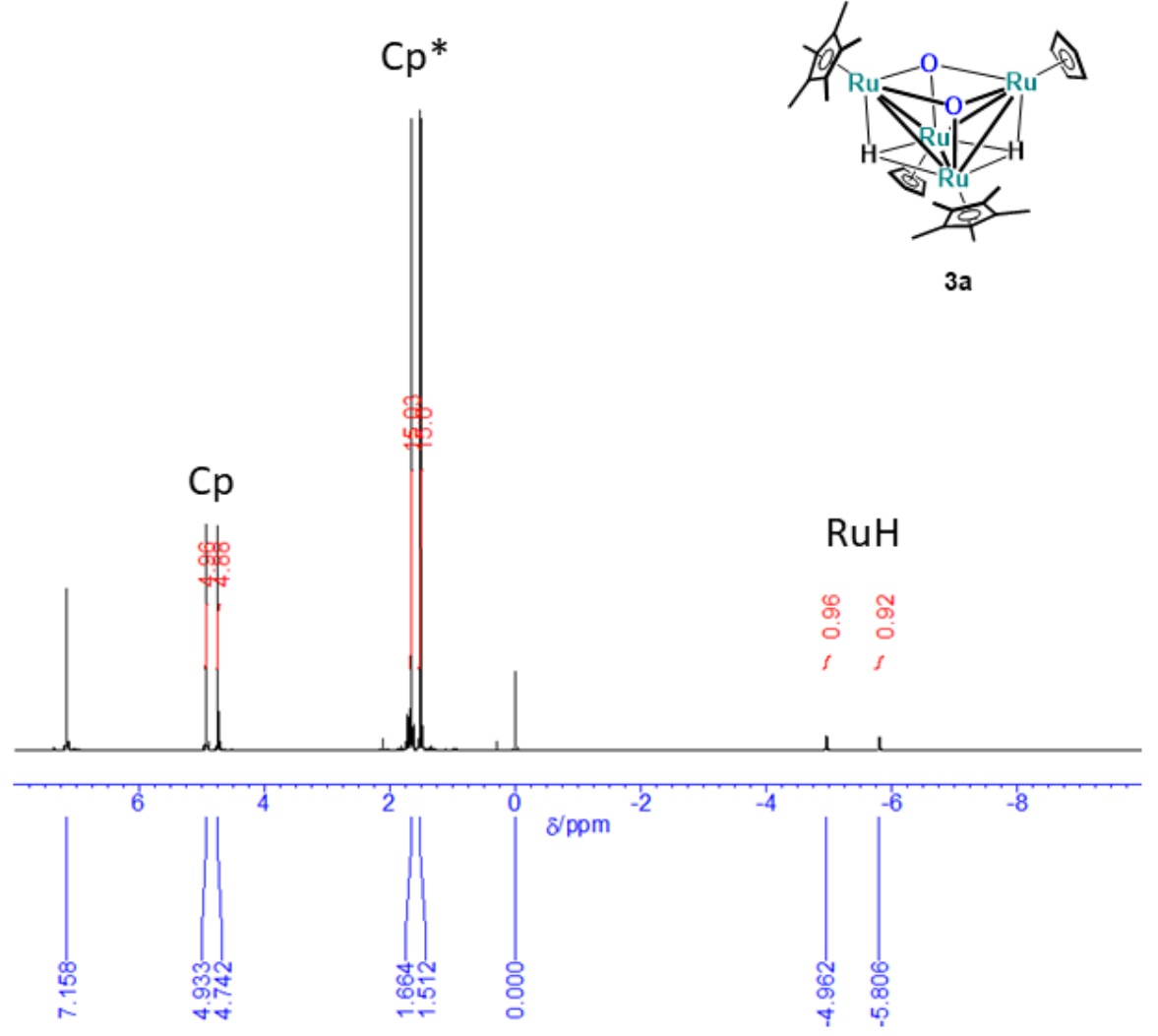

Figure S23 ${ }^{1} \mathrm{H}$ NMR spectrum of $\mathbf{3 a}\left(400 \mathrm{MHz},\left[\mathrm{D}_{6}\right]\right.$ benzene, $\left.25^{\circ} \mathrm{C}\right)$. 


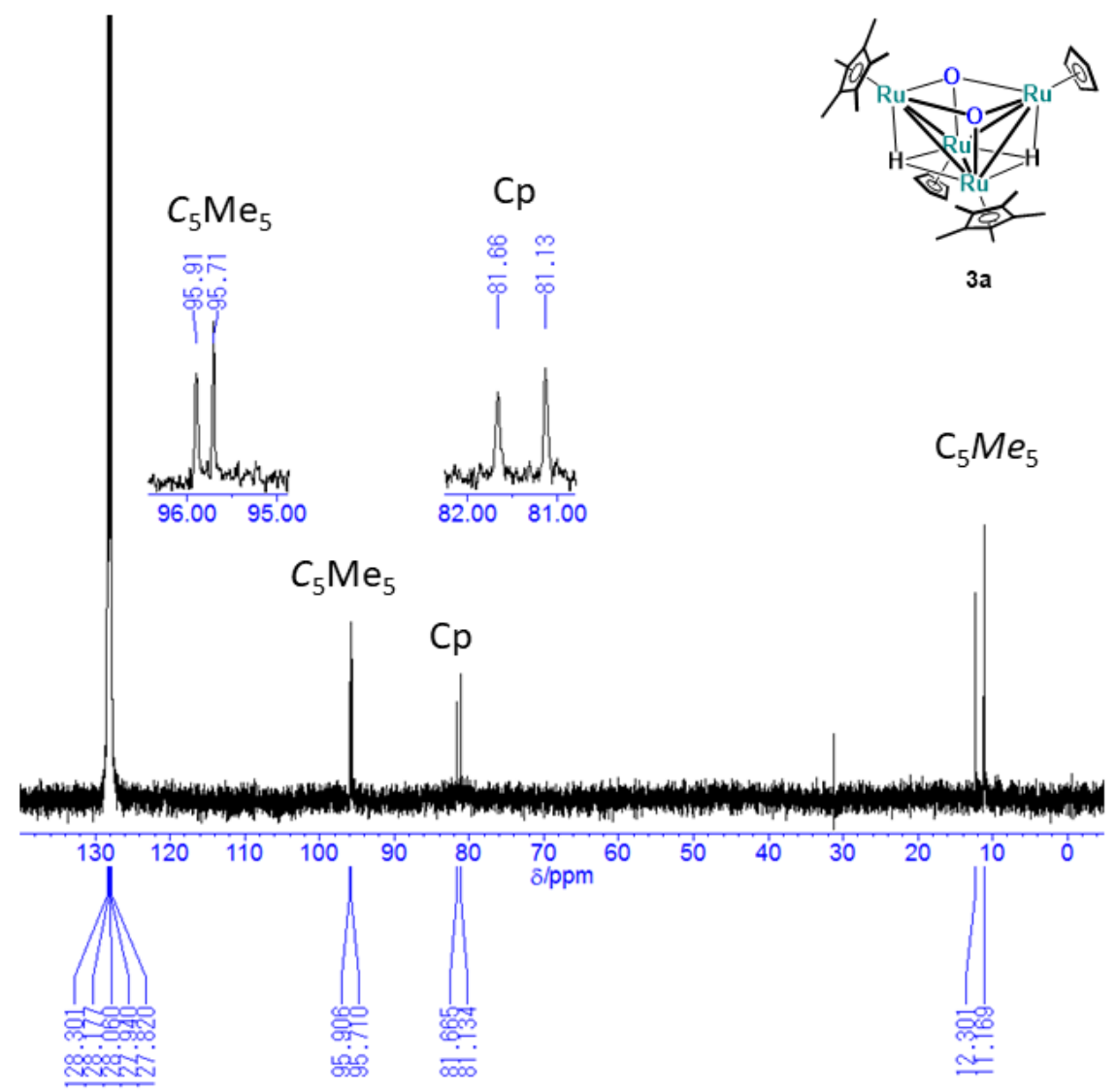

Figure S24 ${ }^{13} \mathrm{C}\left\{{ }^{1} \mathrm{H}\right\}$ NMR spectrum of $\mathbf{3 a}\left(100 \mathrm{MHz},\left[\mathrm{D}_{6}\right]\right.$ benzene, $\left.25{ }^{\circ} \mathrm{C}\right)$.

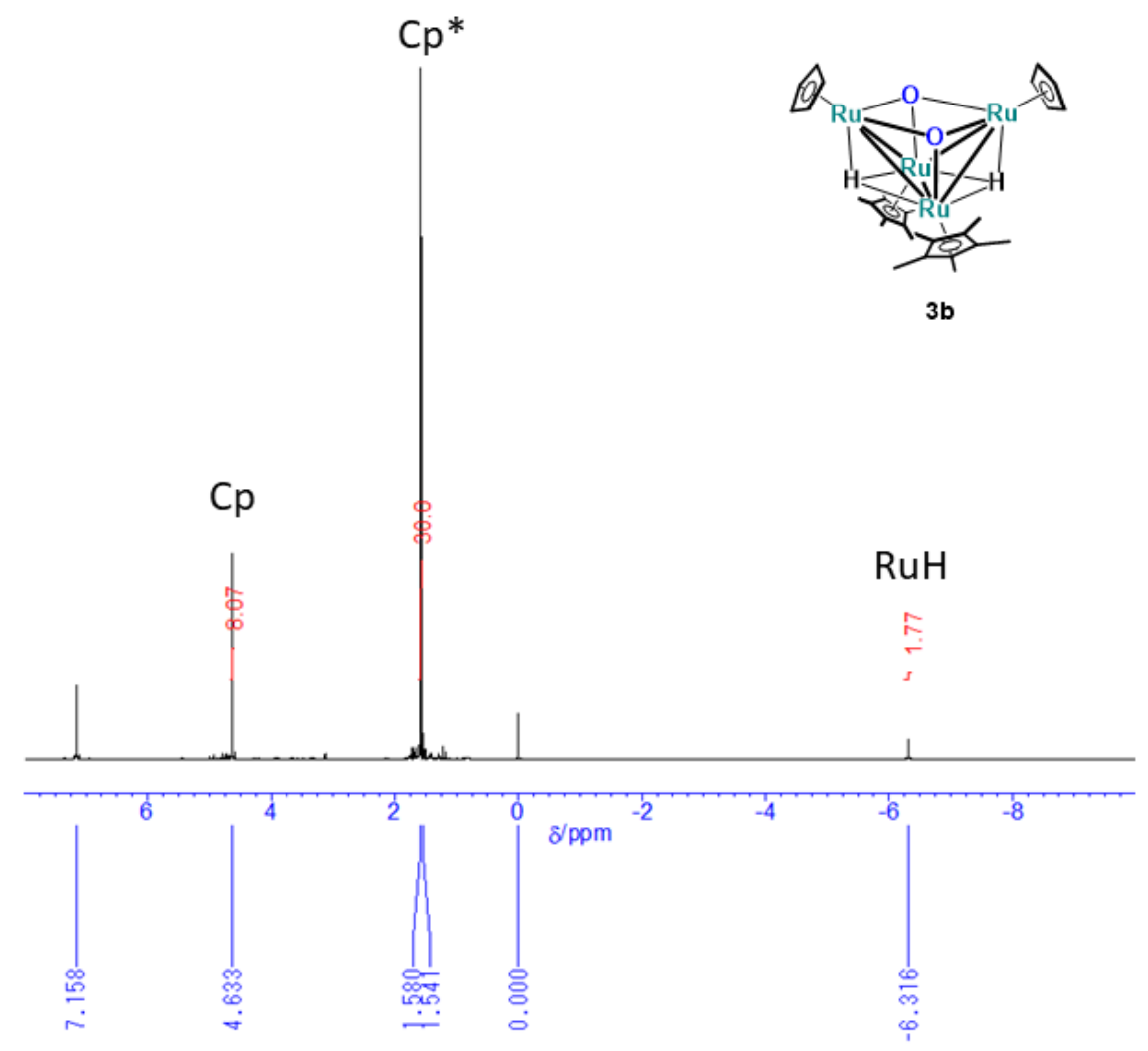

Figure S25 $\quad{ }^{1} \mathrm{H}$ NMR spectrum of $\mathbf{3 b}\left(400 \mathrm{MHz},\left[\mathrm{D}_{6}\right]\right.$ benzene, $\left.25{ }^{\circ} \mathrm{C}\right)$. 


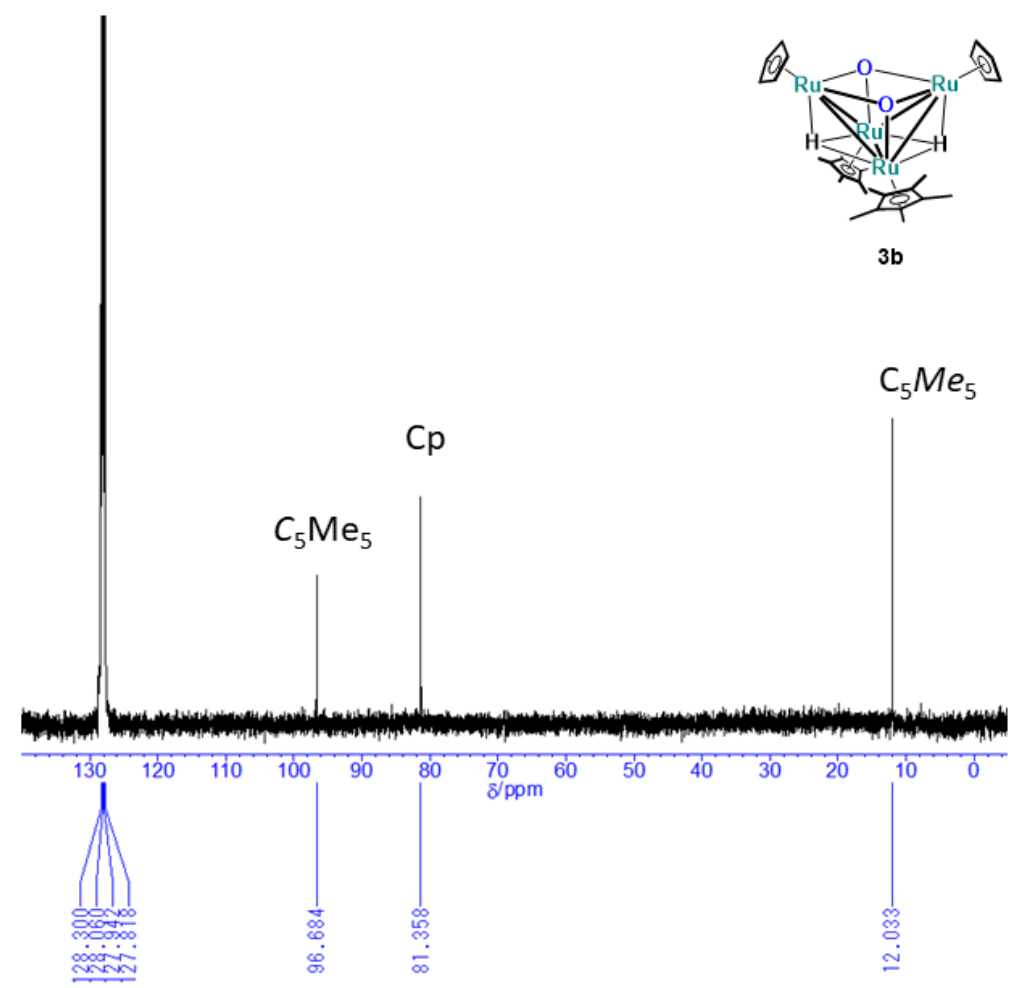

Figure S26 ${ }^{13} \mathrm{C}\left\{{ }^{1} \mathrm{H}\right\}$ NMR spectrum of $\mathbf{3 b}\left(100 \mathrm{MHz},\left[\mathrm{D}_{6}\right]\right.$ benzene, $\left.25{ }^{\circ} \mathrm{C}\right)$.

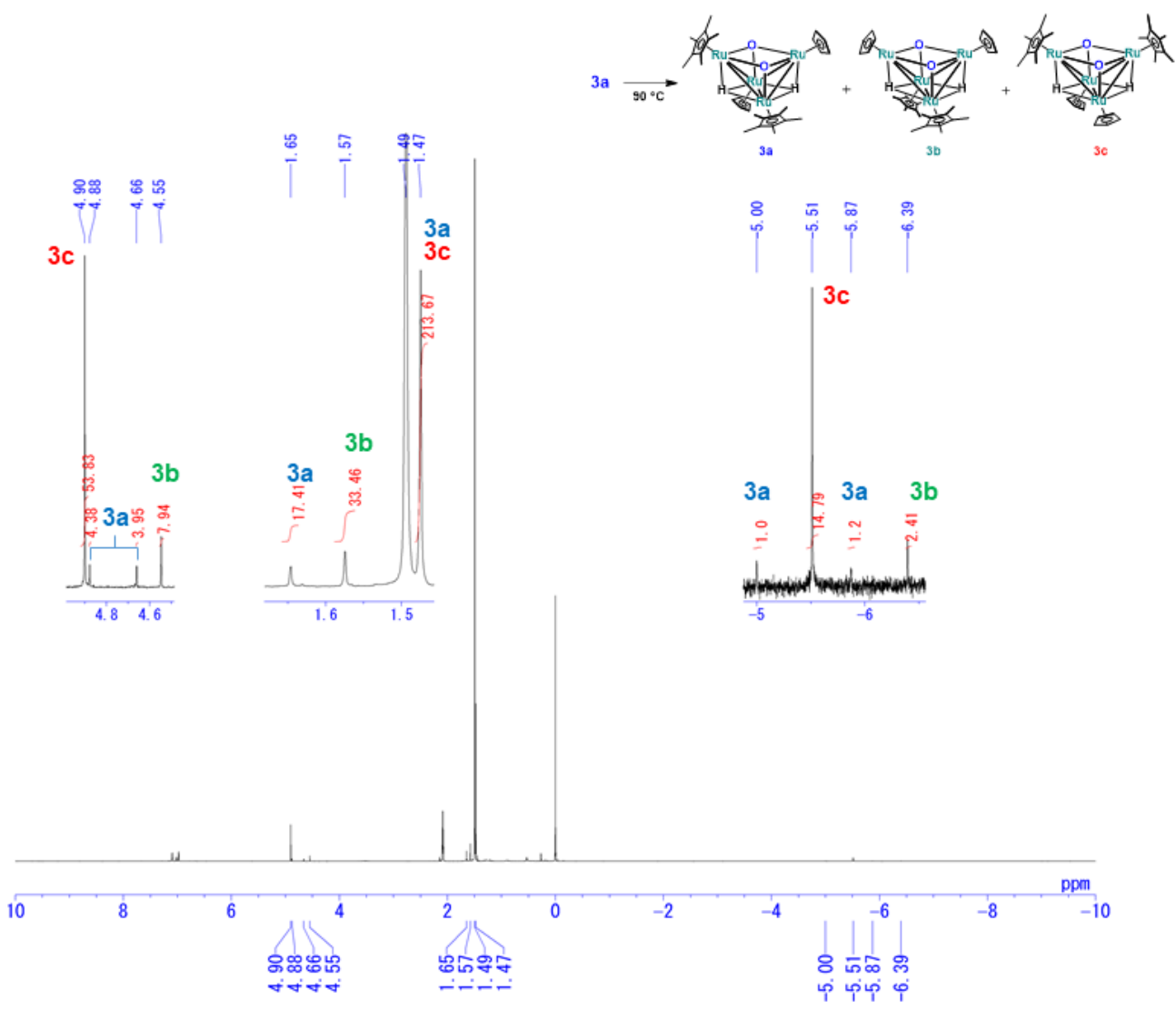

Figure S27 ${ }^{1} \mathrm{H}$ NMR spectrum of the equilibrated mixture of $\mathbf{3 a}, \mathbf{3 b}$, and $\mathbf{3 c}$ obtained by the thermolysis of $3 \mathbf{a}$ at $90{ }^{\circ} \mathrm{C}$ for $12 \mathrm{~h}\left(400 \mathrm{MHz},\left[\mathrm{D}_{8}\right]\right.$ toluene, $\left.25^{\circ} \mathrm{C}\right)$. The singlet resonating at $\delta 1.49$ was derived from cyclooctane used as an internal standard. 


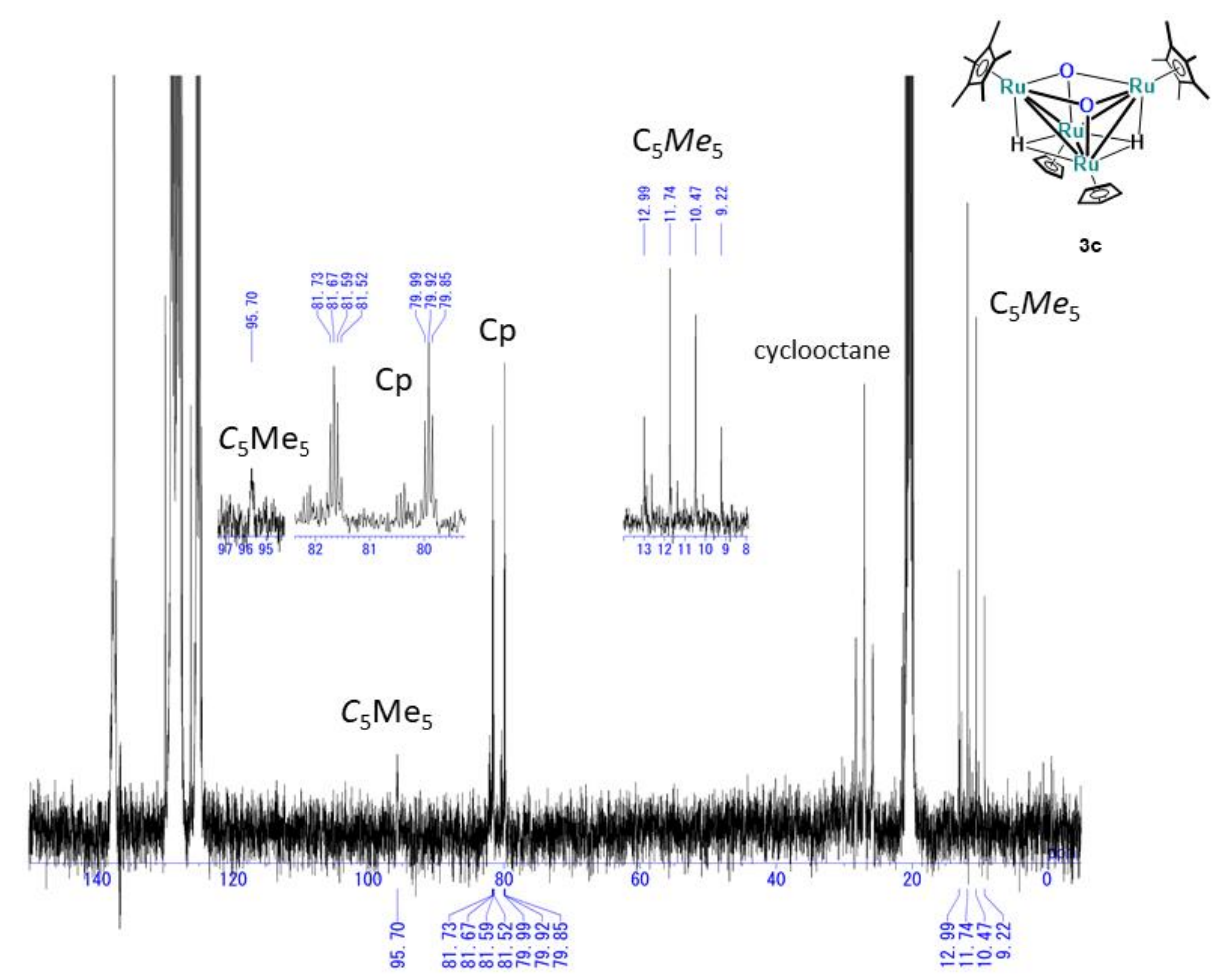

Figure S28 ${ }^{13} \mathrm{C}$ NMR spectrum of $\mathbf{3 c}$ obtained by the thermolysis of $\mathbf{3 a}$ at $90{ }^{\circ} \mathrm{C}$ for $12 \mathrm{~h}(100 \mathrm{MHz}$, $\left[\mathrm{D}_{8}\right]$ toluene, $\left.25^{\circ} \mathrm{C}\right)$.

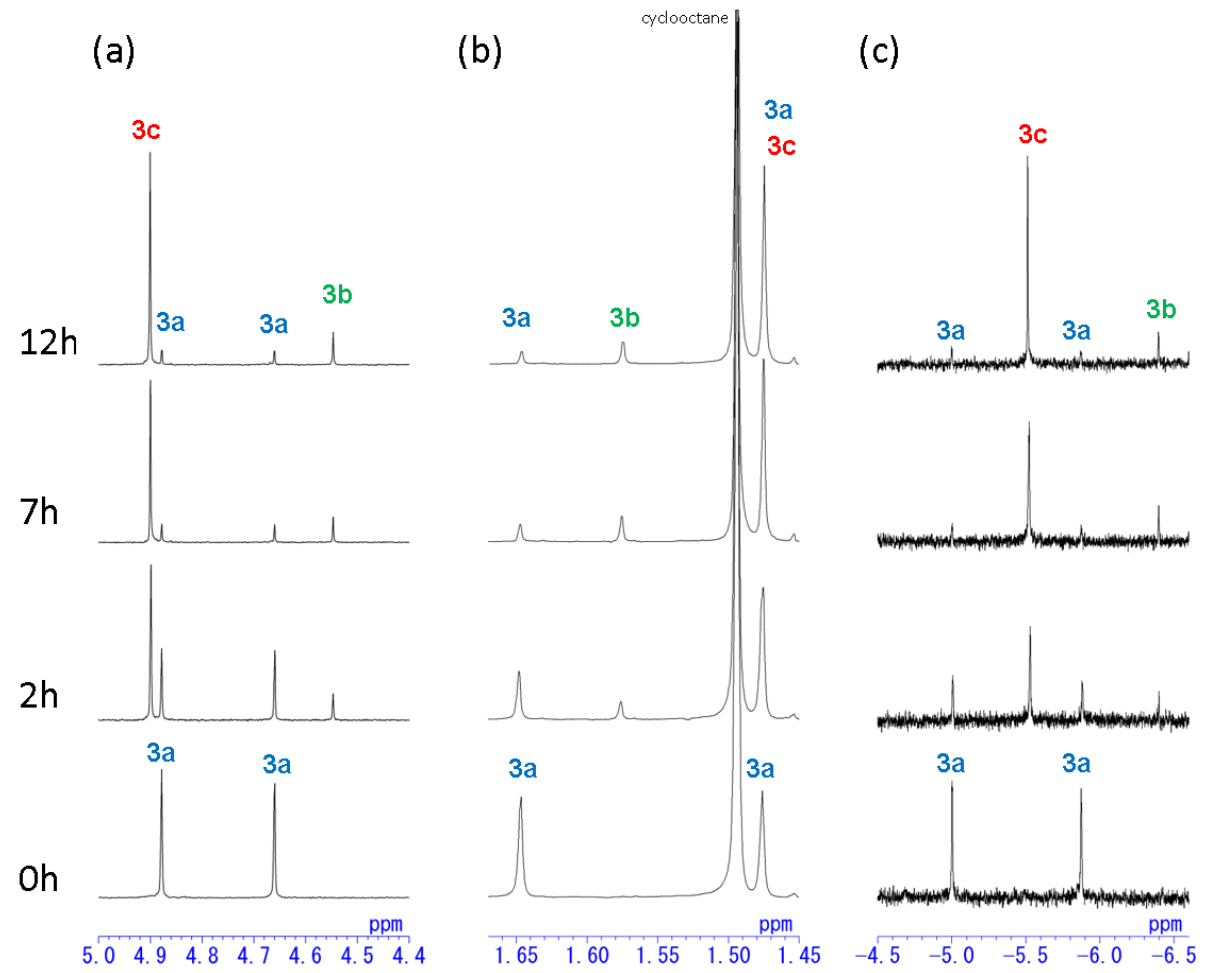

Figure S29 ${ }^{1} \mathrm{H}$ NMR spectra of the mixture of $\mathbf{3 a}, \mathbf{3 b}$, and $\mathbf{3 c}$ obtained upon thermolysis of $\mathbf{3 a}$ for 2,7 , and 12 hours, showing (a) $\mathrm{Cp}$, (b) $\mathrm{Cp}^{*}$, and (c) hydrido regions ( $400 \mathrm{MHz},\left[\mathrm{D}_{8}\right]$ toluene, $25^{\circ} \mathrm{C}$ ). The singlet resonating at $\delta 1.49$ was derived from cyclooctane used as an internal standard. 


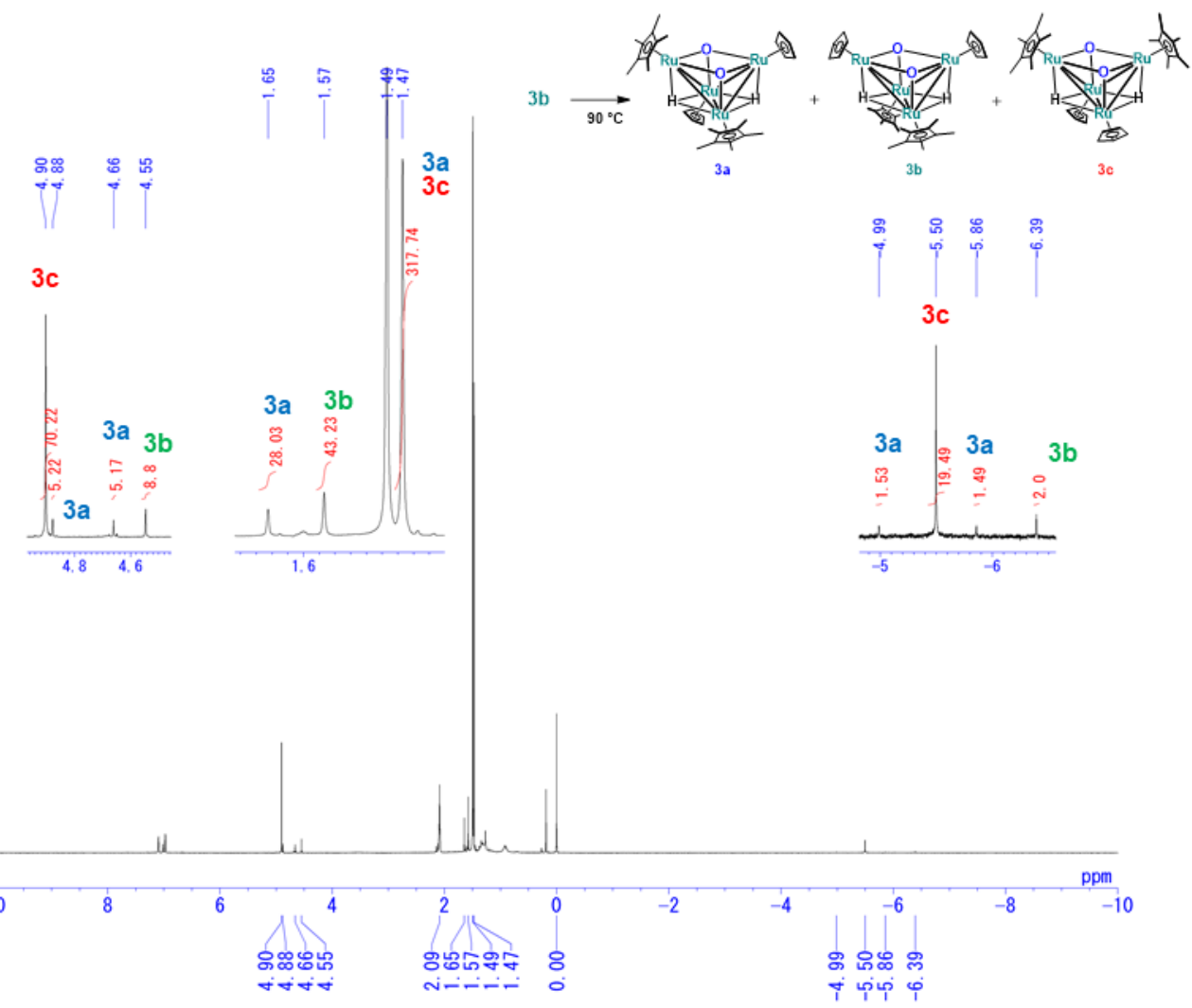

Figure S30 ${ }^{1} \mathrm{H}$ NMR spectrum of the equilibrated mixture of $\mathbf{3 a}, \mathbf{3 b}$, and $\mathbf{3} \mathbf{c}$ obtained by the thermolysis of $\mathbf{3 b}$ at $90{ }^{\circ} \mathrm{C}$ for $60 \mathrm{~h}\left(400 \mathrm{MHz},\left[\mathrm{D}_{8}\right]\right.$ toluene, $\left.25{ }^{\circ} \mathrm{C}\right)$. The singlet resonating at $\delta 1.49$ was derived from cyclooctane used as an internal standard.

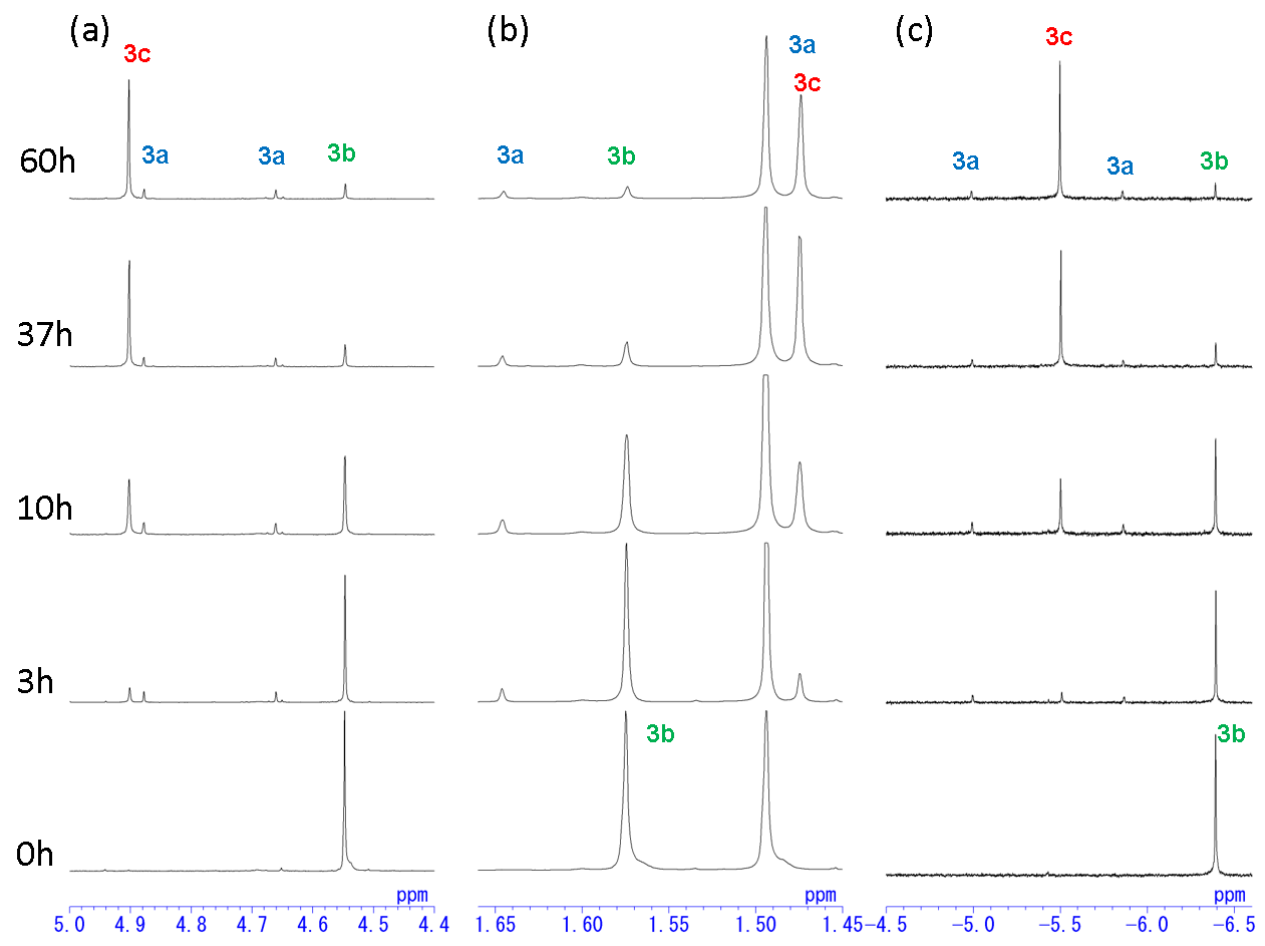

Figure S31 ${ }^{1} \mathrm{H}$ NMR spectra of the mixture of $\mathbf{3 a}, \mathbf{3 b}$, and $\mathbf{3 c}$ obtained by the thermolysis of $\mathbf{3 b}$ for 3, 10, 37 , and 60 hours, showing (a) $\mathrm{Cp}$, (b) $\mathrm{Cp}^{*}$, and (c) hydrido regions (400 MHz, [ $\left.\mathrm{D}_{8}\right]$ toluene, $25^{\circ} \mathrm{C}$ ). The singlet resonating at $\delta 1.49$ was derived from cyclooctane used as an internal standard. 


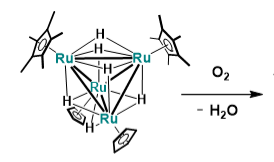

2b

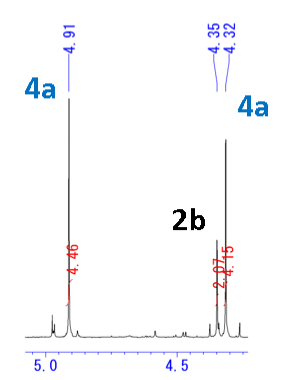

Cp
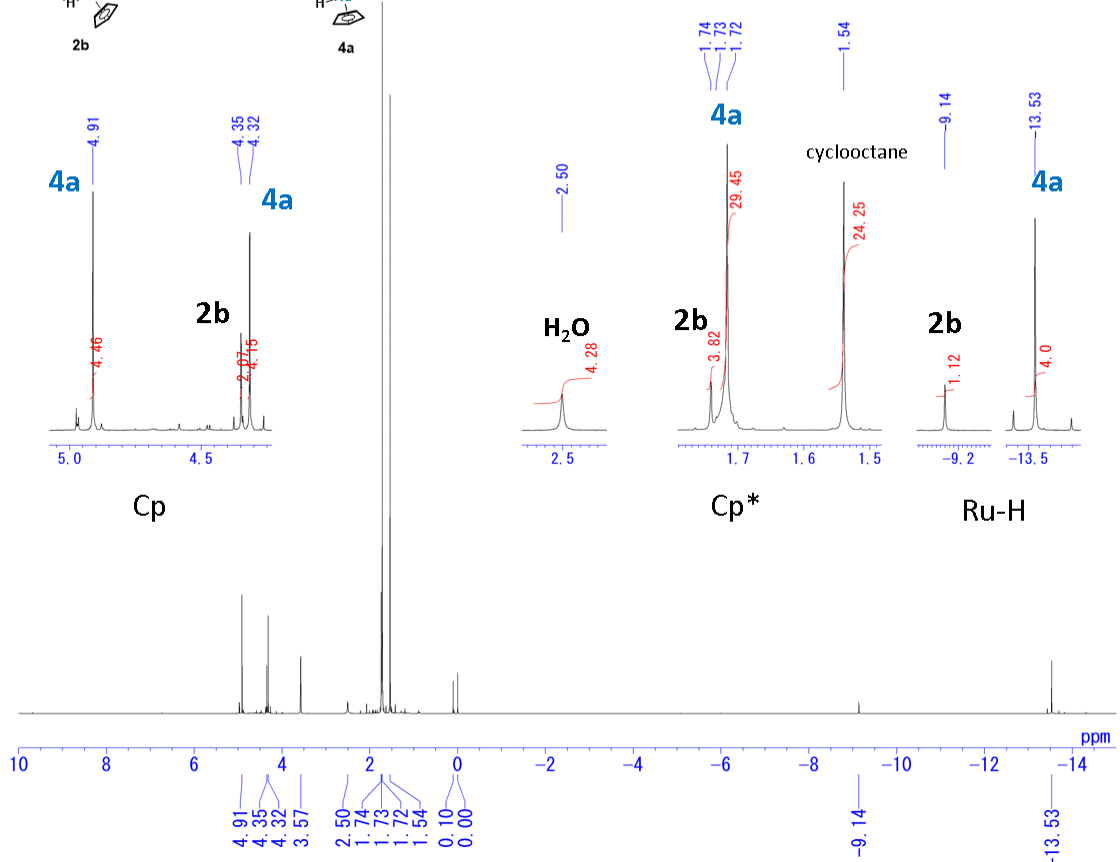

Figure S32 ${ }^{1} \mathrm{H}$ NMR spectrum of $4 \mathbf{a}$ obtained by the reaction of $\mathbf{2 b}$ with $1 \mathrm{~atm}$ of $\mathrm{O}_{2}$ at $25{ }^{\circ} \mathrm{C}$ for $22 \mathrm{~h}(400$ $\mathrm{MHz},\left[\mathrm{D}_{8}\right]$ tetrahydrofuran, $\left.25^{\circ} \mathrm{C}\right)$. The singlet resonating at $\delta 1.54$ was derived from cyclooctane used as an internal standard.

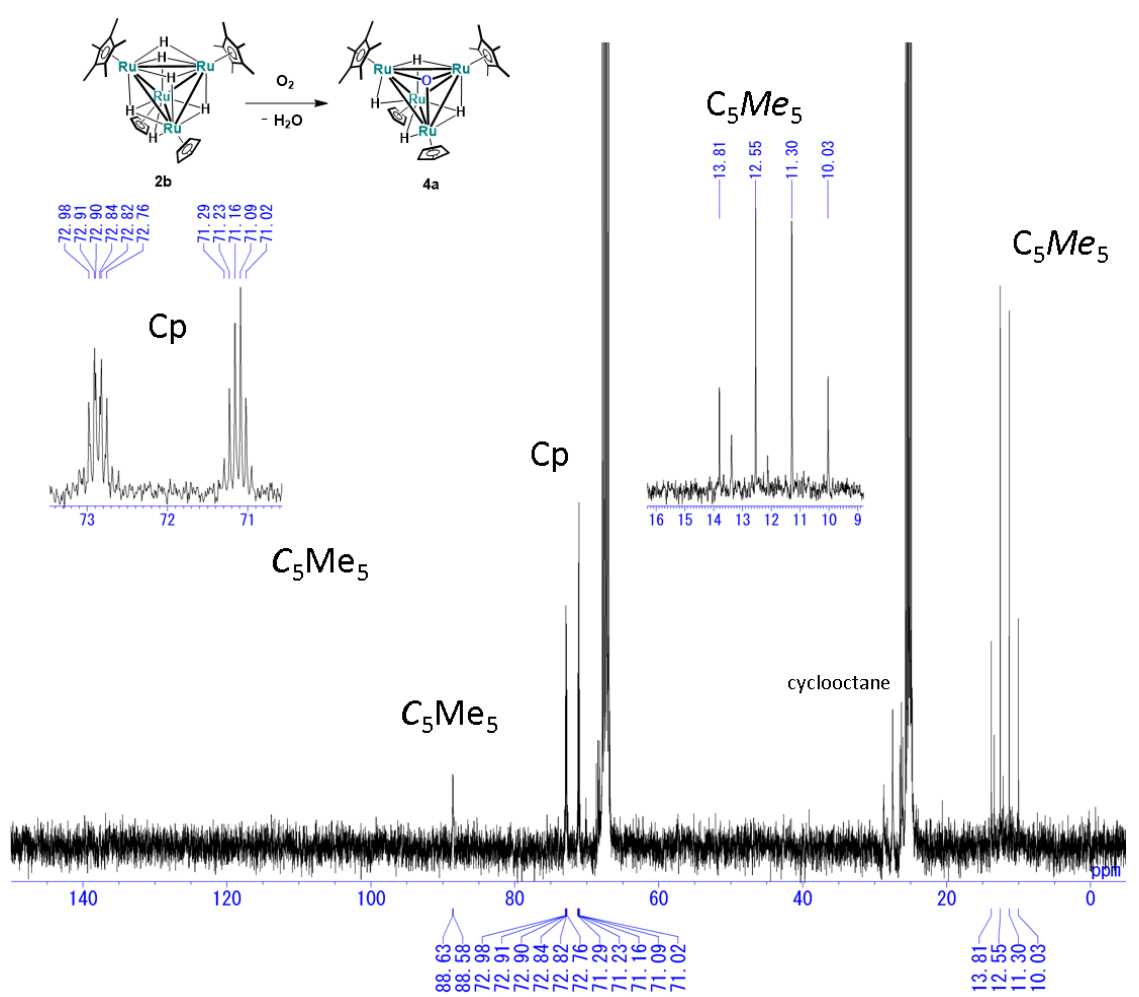

Figure S33 ${ }^{13} \mathrm{C}$ NMR spectrum of $\mathbf{4 a}$ obtained by the reaction of $\mathbf{2 b}$ with 1 atm of $\mathrm{O}_{2}$ at $25{ }^{\circ} \mathrm{C}$ for $22 \mathrm{~h}$ $\left(100 \mathrm{MHz},\left[\mathrm{D}_{8}\right]\right.$ tetrahydrofuran, $\left.25^{\circ} \mathrm{C}\right)$. The triplet resonating at $\delta 27.5\left(J_{\mathrm{CH}}=124 \mathrm{~Hz}\right)$ was derived from cyclooctane used as an internal standard. 


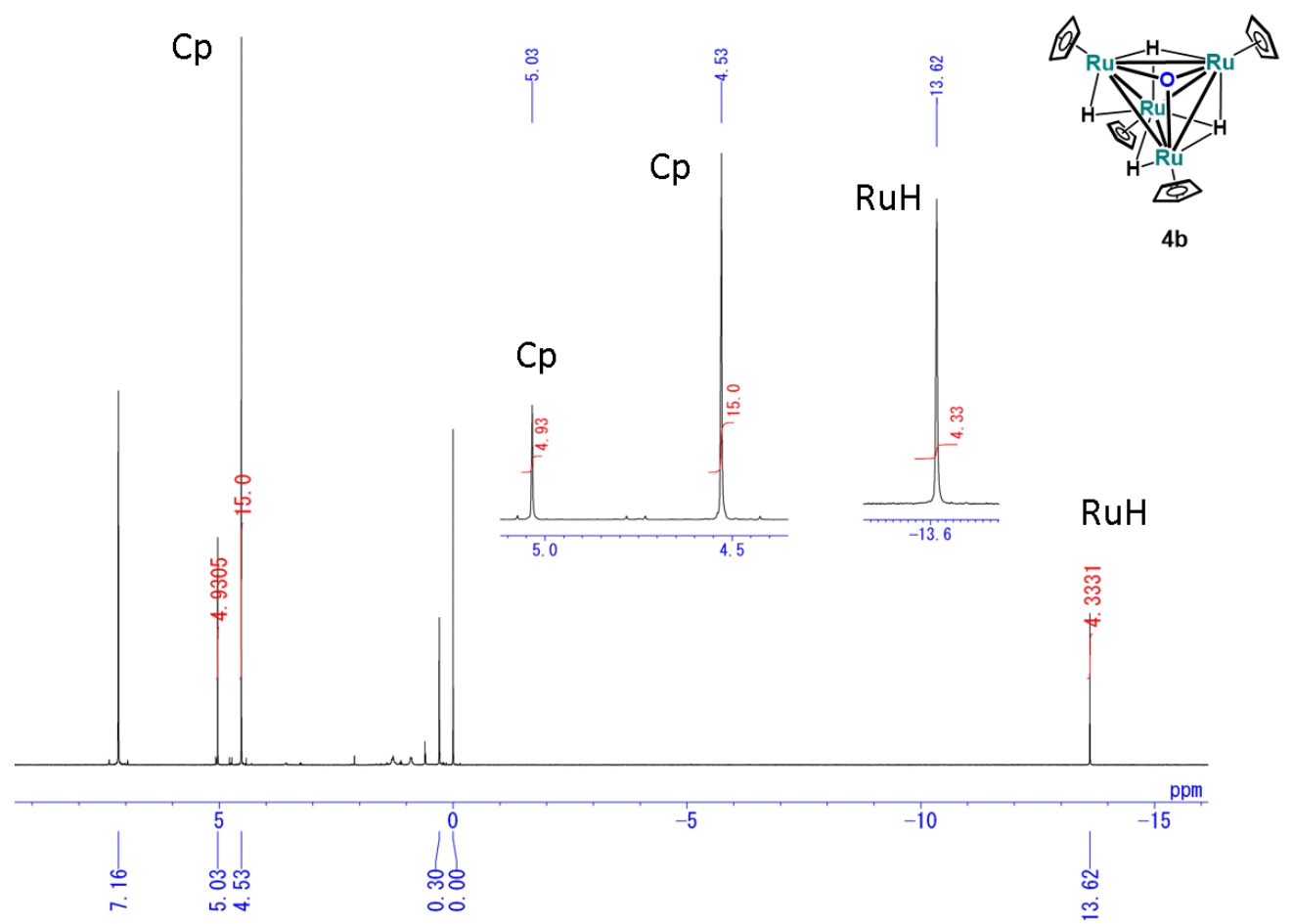

Figure S34 ${ }^{1} \mathrm{H}$ NMR spectrum of $\mathbf{4 b}\left(400 \mathrm{MHz},\left[\mathrm{D}_{6}\right]\right.$ benzene, $\left.25^{\circ} \mathrm{C}\right)$.

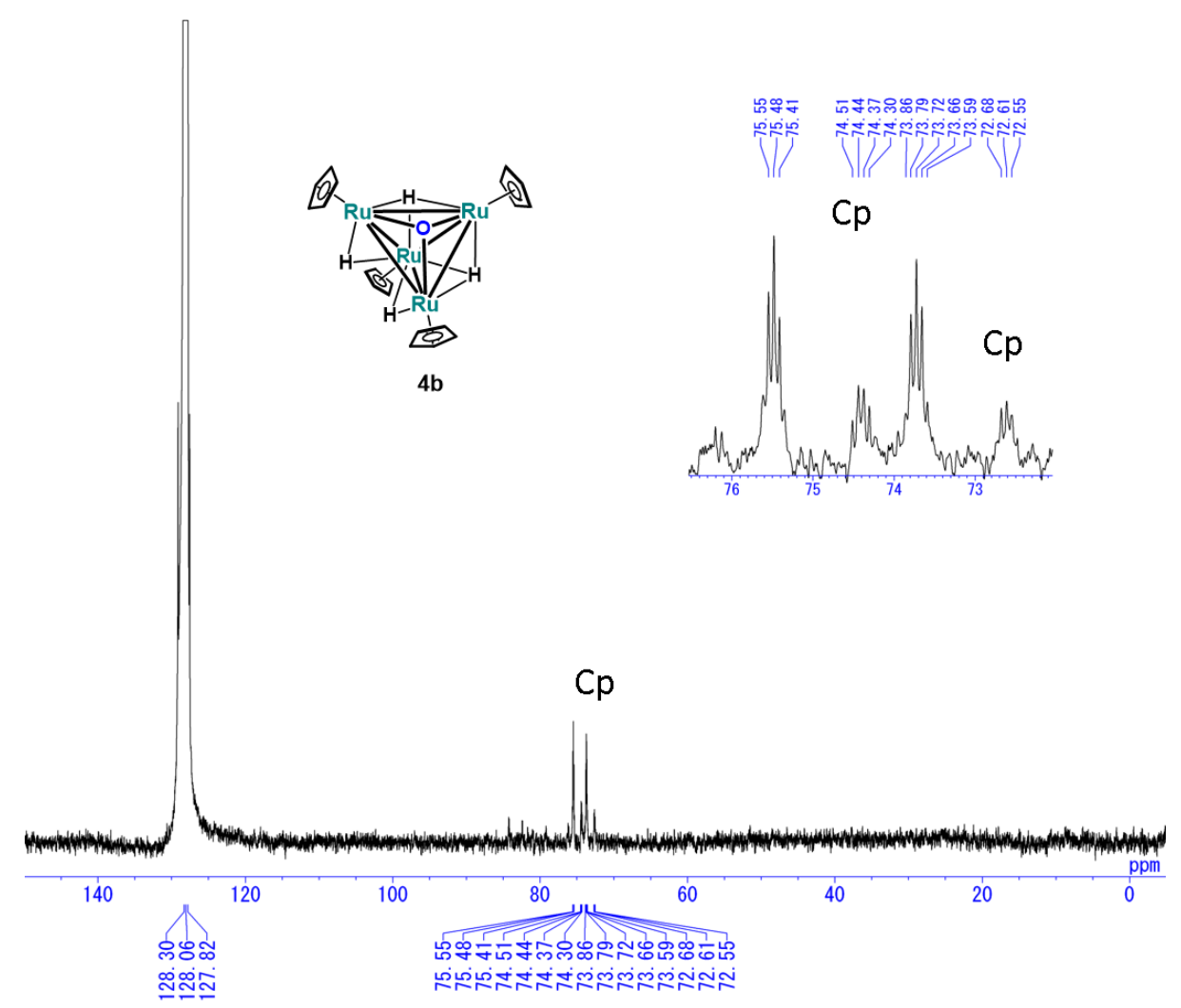

Figure S35 ${ }^{13} \mathrm{C}$ NMR spectrum of $\mathbf{4 b}\left(100 \mathrm{MHz},\left[\mathrm{D}_{6}\right]\right.$ benzene, $\left.25^{\circ} \mathrm{C}\right)$. 


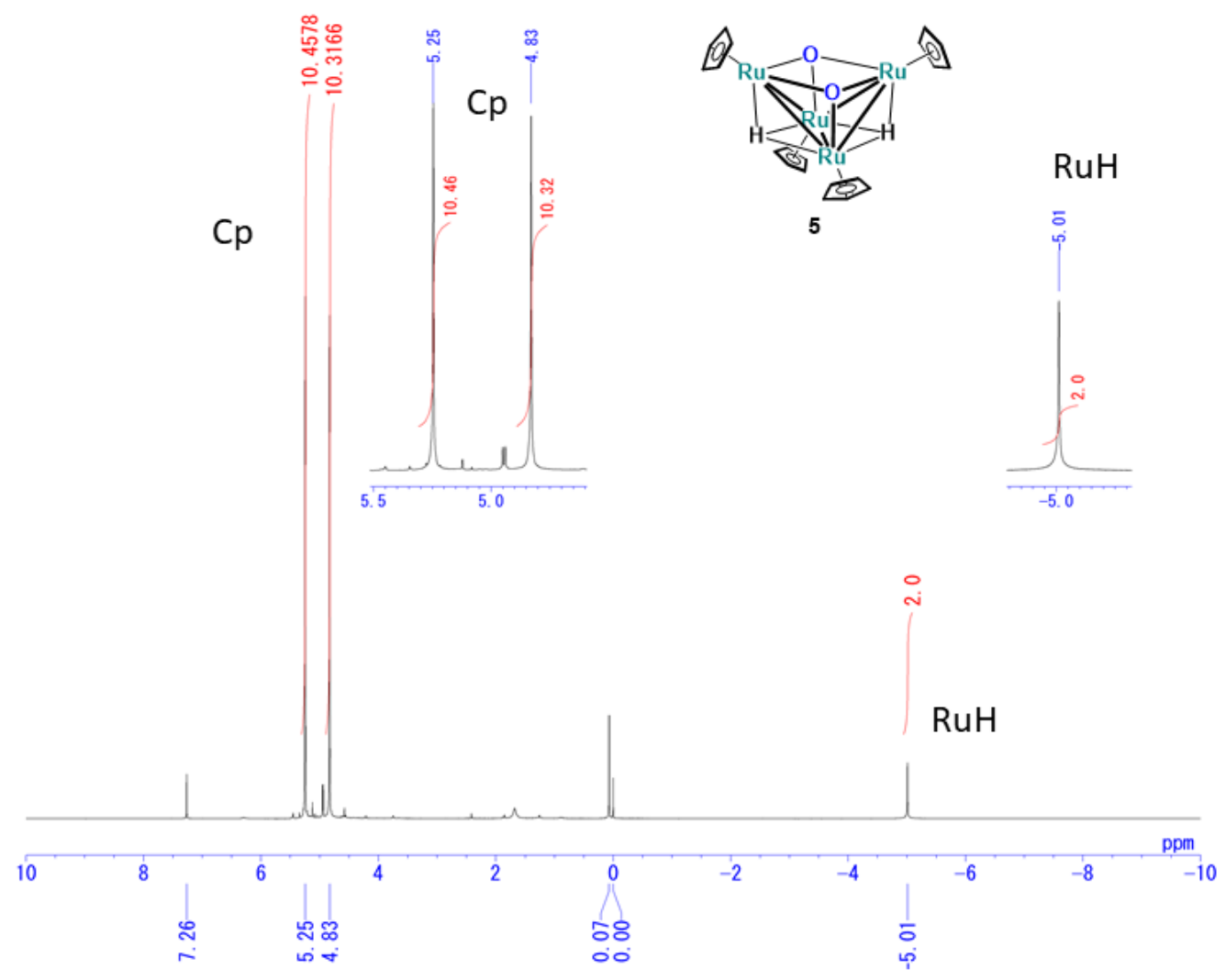

Figure S36 ${ }^{1} \mathrm{H}$ NMR spectrum of 5 (400 MHz, [D]chloroform, $25^{\circ} \mathrm{C}$ ).

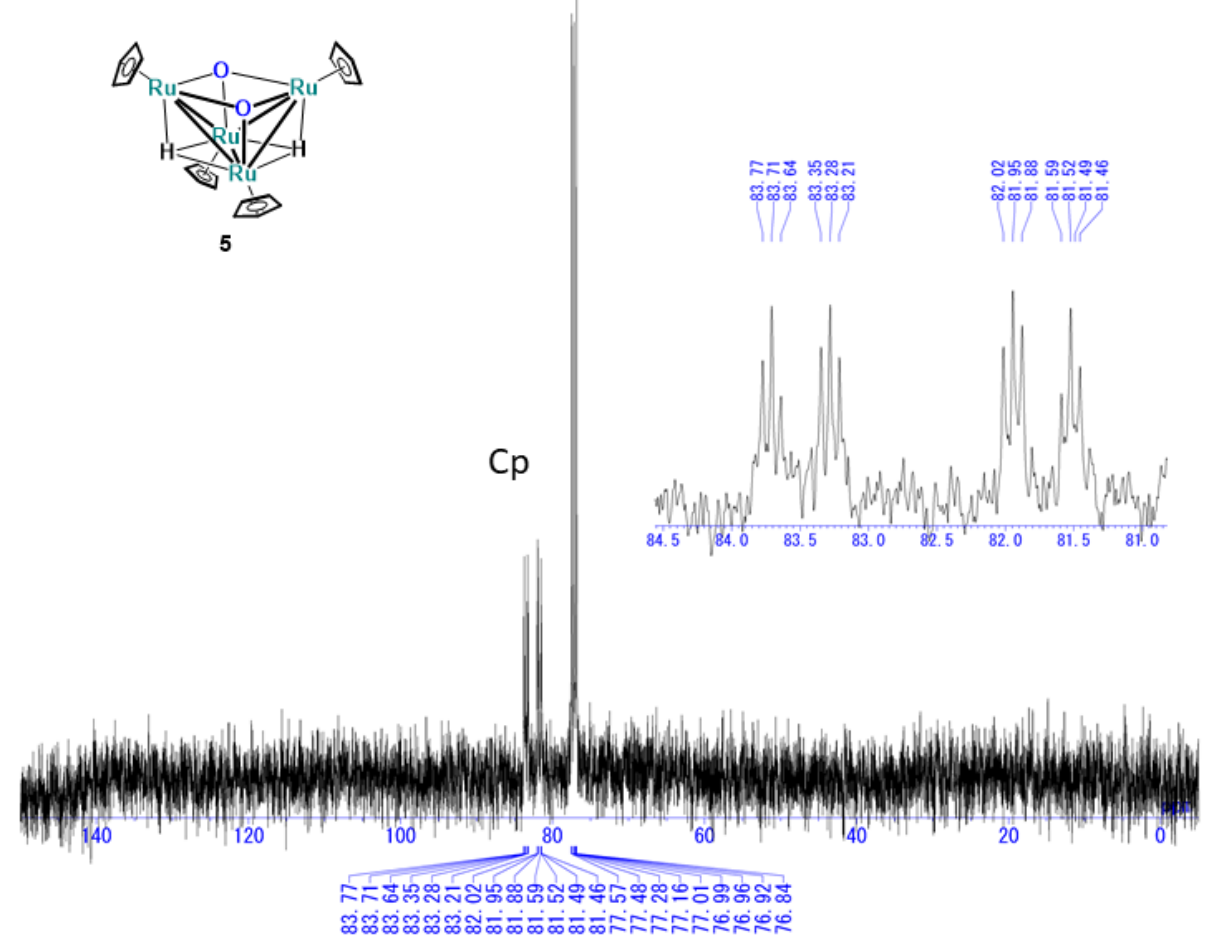

Figure S37 ${ }^{13} \mathrm{C}$ NMR spectrum of $5\left(100 \mathrm{MHz},[\mathrm{D}]\right.$ chloroform, $\left.25{ }^{\circ} \mathrm{C}\right)$. 


\section{Reference}

(1) Kameo, H.; Ito, Y.; Shimogawa, R.; Koizumi, A.; Chikamori, H.; Fujimoto, J.; Suzuki, H.; Takao, T. Synthesis and Characterisation of Tetranuclear Ruthenium Polyhydrido Clusters with Pseudo-Tetrahedral Geometry. Dalton Trans. 2017, 46, 5631-5643. 